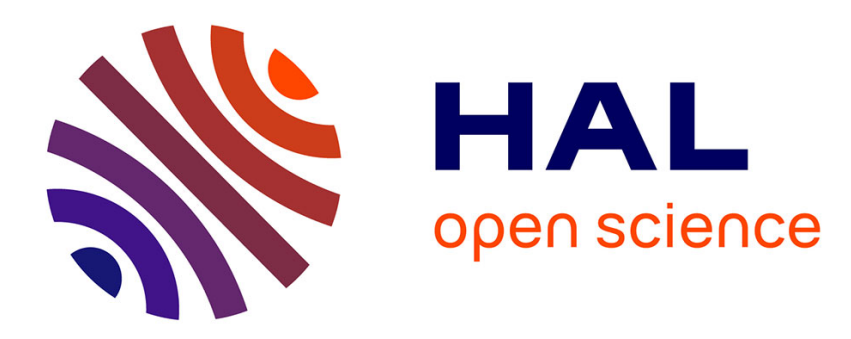

\title{
Coupled dynamics of Earth's geomagnetic westward drift and inner core super-rotation \\ Guillaume Pichon
}

\section{To cite this version:}

Guillaume Pichon. Coupled dynamics of Earth's geomagnetic westward drift and inner core superrotation. Earth Sciences. Université Sorbonne Paris Cité, 2017. English. NNT: 2017USPCC271. tel-02169714

\section{HAL Id: tel-02169714 \\ https://theses.hal.science/tel-02169714}

Submitted on 1 Jul 2019

HAL is a multi-disciplinary open access archive for the deposit and dissemination of scientific research documents, whether they are published or not. The documents may come from teaching and research institutions in France or abroad, or from public or private research centers.
L'archive ouverte pluridisciplinaire HAL, est destinée au dépôt et à la diffusion de documents scientifiques de niveau recherche, publiés ou non, émanant des établissements d'enseignement et de recherche français ou étrangers, des laboratoires publics ou privés. 


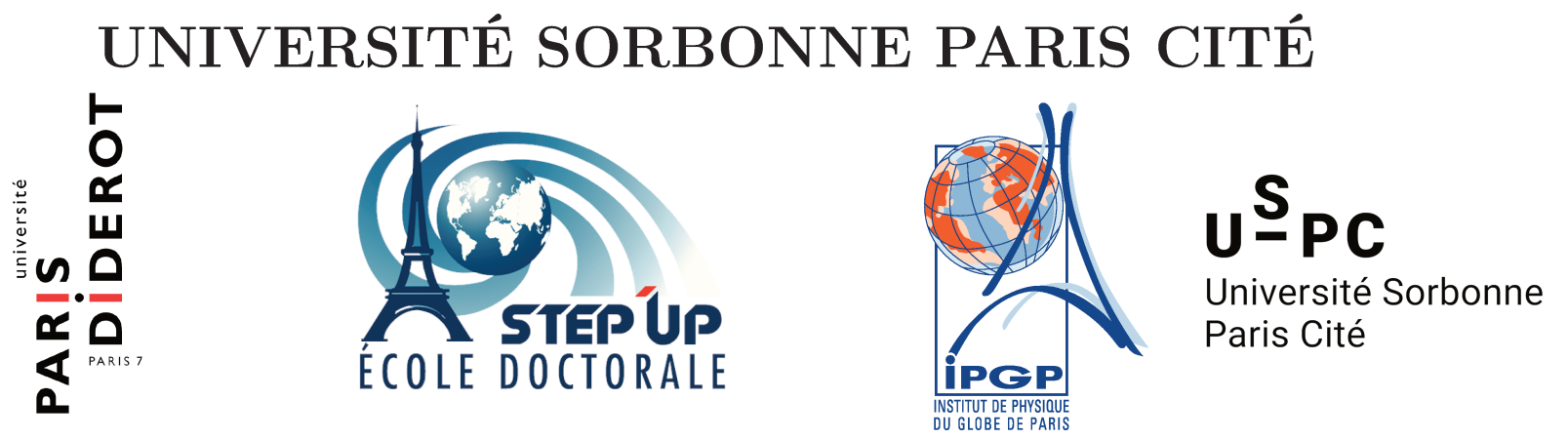

Thèse préparée à

l'INSTITUT DE PHYSIQUE DU GLOBE DE PARIS

École doctorale STEP'UP- ED N ${ }^{\circ} 560$

IPGP - Équipe de dynamique des fluides géologiques

\section{Dynamique rotationnelle couplée de la dérive géomagnétique vers l'ouest et de la super-rotation de la graine terrestre}

\section{par \\ Guillaume Pichon}

présentée et soutenue publiquement le

21 décembre 2017

Thèse de doctorat de Sciences de la Terre et de l'environnement

dirigée par Julien Aubert

\& Alexandre Fournier

devant un jury composé de :

Dominique Jault Rapporteur

Directeur de recherche (ISTerre - CNRS)

Céline Guervilly Rapportrice

Lecturer (Newcastle University)

Marianne Greff Présidente du jury et examinatrice

Professeur (IPGP - Université Paris Diderot)

Renaud Deguen Examinateur

Maître de conférence (LGLTPE - université de Lyon 1)

Alexandre Fournier Co-Directeur de thèse

Professeur (IPGP - Université Paris Diderot)

Julien Aubert Directeur de thèse

Directeur de recherche (IPGP - CNRS) 



\title{
PhD Thesis
}

Coupled dynamics of Earth's geomagnetic westward drift and inner core super-rotation

\author{
by \\ Guillaume Pichon
}

Institut de physique du globe de Paris

Université Sorbonne Paris Cité École doctorale STEP'UP 



\section{Remerciements}

Cette thèse n'aurait pas pu voir le jour sans le support de l'IPGP et de ses membres. Pour cette raison, je tiens tout d'abord à remercier Claude Jaupart qui, en plus de m'avoir donné goût à la dynamique des fluides géologiques m'a suivit tout au long de mes études : des cours de géosciences en première année jusqu'à ce coup de main pour porter les courses du pot, le matin même de ma soutenance. Pour des raisons similaires je tiens a remercier Edouard Kaminski, pour m'avoir donné envie de faire de la recherche en encadrant mes stages de L3 et M1. Je le remercie également pour son soutien à l'obtention de cette thèse ainsi qu'aux associations étudiantes, qui m'a permis d'explorer l'île de Sao Miguel aux Açores, avec l'AESTP7. Parmi les professeurs de l'IPGP je tiens aussi à remercier Marianne Greff, pour son enseignement, son soutien à cette thèse, son accompagnement au cours de celle-ci et sa présidence du jury de soutenance, que je remercie également pour leur présence et leurs critiques, bonnes ou mauvaises.

Plus personnellement, je remercie mes amis, qui se reconnaitront, les doctorants de l'IPGP et l'équipe de dynamique des fluides géologiques pour l'accueil, l'ambiance, les repas, les pauses et autres à cotés qui font de cet endroit un formidable espace de travail et de vie. Je remercie également mes parents, pour avoir trouvé le juste équilibre entre l'encadrement, le soutien et la liberté pour moi de faire mes propres choix d'avenir.

Enfin, mes remerciements les plus importants sont adressés à Julien et Alexandre, pour tout ce qu'ils ont fait pour moi, avant, pendant et même après la soutenance de cette thèse. Je n'ai pas été un étudiant modèle au cours de ces trois années, et si les réussites de cette thèse sont en grande partie de leur fait, il n'en est pas de même pour ses échecs. Merci pour votre patience, votre aide et votre bonne humeur. Merci pour tout, vraiment.

Lou, merci. 

1 Introduction $\quad 9$

1.1 Prologue .............................. 9

1.1.1 De l'aimant à la boussole . . . . . . . . . . . . . . . . . . . . 99 9

1.1.2 Premières observations . . . . . . . . . . . . . . . . . . . . . . . 10

1.1.3 Premières théories . . . . . . . . . . . . . . . . . . 10

1.1.4 Intérieur de la Terre . . . . . . . . . . . . . . . . . . . . . . 11

1.1.5 La géodynamo . . . . . . . . . . . . . . . . . . . . . 13

1.1.6 Simulations numériques . . . . . . . . . . . . . . 14

1.1.7 Progrès et objectif de la thèse . . . . . . . . . . . . . . . . 15

1.2 Earth's core structure and dynamics . . . . . . . . . . . . . . . . . 16

1.2.1 Internal structure of the Earth . . . . . . . . . . . . . . . 16

1.2.2 Dynamics of the core . . . . . . . . . . . . . . . 17

1.3 Earth's magnetic field . . . . . . . . . . . . . . . . . . . . . . . 18

1.3.1 Geomagnetic observations . . . . . . . . . . . . . . . . 19

1.3.2 Other sources . . . . . . . . . . . . . . . 21

1.3.3 Magnetic field models . . . . . . . . . . . . . . . . . 22

1.4 The geomagnetic westward drift . . . . . . . . . . . . . . . . . . 25

1.5 Inner core super-rotation . . . . . . . . . . . . . . . . . . . 29

1.5.1 Dynamics . . . . . . . . . . . . . . . . . . . . 29

1.5.2 Seismological evidence . . . . . . . . . . . . . . . . . 33

1.6 Objectives of this work . . . . . . . . . . . . . . . . . . 35

1.6.1 Coupled-Earth dynamo . . . . . . . . . . . . . . . 35

1.6.2 Direct core-mantle coupling . . . . . . . . . . . . . . 36

1.6.3 $\mathrm{PhD}$ work . . . . . . . . . . . . . . . . . . . 39

2 Model $\quad 43$

2.1 Geometry .............................. 44

2.2 Conservation laws . . . . . . . . . . . . . . . . . . . . 44

2.2 .1 Mass . . . . . . . . . . . . . . . . . . . . 44

2.2.2 Momentum . . . . . . . . . . . . . . . . . 45

2.2.3 Angular momentum . . . . . . . . . . . . . . 46 
2.2.4 Energy . . . . . . . . . . . . . . . . . . 47

2.2 .5 Induction equation . . . . . . . . . . . . . . 50

2.2.6 Dimensioned system . . . . . . . . . . . . . . . . . . . 51

2.2.7 Boundary conditions . . . . . . . . . . . . . . . . 53

2.3 Dimensionless equations . . . . . . . . . . . . . . . . . . 54

2.3.1 Viscous scaling . . . . . . . . . . . . . . . . 54

2.3.2 Diffusion-free scaling . . . . . . . . . . . . . . . . . 55

2.3.3 Core regime . . . . . . . . . . . . . . . . 56

2.3.4 Geodynamo simulation regime . . . . . . . . . . . . 57

3 Numerical implementation $\quad 61$

3.1 Parody-JA Code . . . . . . . . . . . . . . . . . . 62

3.1.1 Toroidal-Poloidal decomposition . . . . . . . . . . . . . . 62

3.1.2 Radial discretization . . . . . . . . . . . . . . . . 63

3.1.3 Spherical harmonics expansion . . . . . . . . . . . . . . . 64

3.1.4 Boundary conditions . . . . . . . . . . . . . . . 65

3.1 .5 Time integration scheme . . . . . . . . . . . . . . 67

3.2 Parody-GP Code . . . . . . . . . . . . . . . . . . . 67

3.2.1 Torque components . . . . . . . . . . . . . . . . 72

3.2.2 Time-dependent gravitational torque . . . . . . . . . . . . . 73

4 Long-term rotational dynamics $\quad 77$

4.1 Introduction . . . . . . . . . . . . . . . . . . . . . . . . 78

4.2 Model . . . . . . . . . . . . . . . . . . . . . . . . . 81

4.2 .1 Conservation laws . . . . . . . . . . . . . . . . . . 81

4.2.2 Dimensionless equations and numerical implementation . . . . . . . 84

4.2.3 Theoretical analysis of the long-term rotational state . . . . . . . . 87

4.2.4 Thermal wind scaling of the convective shear $\mathcal{C}_{\mathrm{f}} \ldots \ldots$. . . . . . . 90

4.3 Results . . . . . . . . . . . . . . . . . . . . . . . . . 91

4.3.1 Typical long-term state of differential rotations . . . . . . . . . 91

4.3.2 Thermal wind scaling of $\mathcal{C}_{\mathrm{f}} \ldots \ldots \ldots \ldots$. . . . . . . . . 94

4.3.3 Long-term electromagnetic torques . . . . . . . . . . . . . . . 94

4.3.4 Link between $\mathcal{S}$ and $\mathcal{D} \ldots \ldots \ldots$. . . . . . . . . . . . 97

4.4 Discussion . . . . . . . . . . . . . . . . . . . . . . . . . . . . . . . . . 99

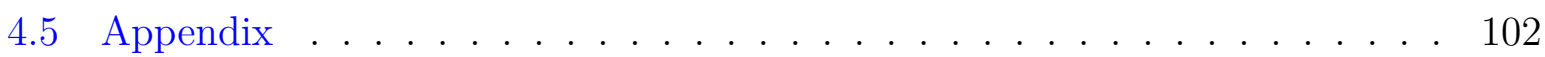

4.5.1 Assessment of numerical uncertainties on torques . . . . . . . . . 102

4.5.2 Statistical convergence . . . . . . . . . . . . . 103

5 Time-dependent electromagnetic torques $\quad 105$

5.1 Diffusion of a perturbation of the magnetic field . . . . . . . . . . . . 106

5.1 .1 General arguments . . . . . . . . . . . . . . . 106 
5.1.2 Mantle-side: Constant conductivity . . . . . . . . . . . . . . . . 111

5.1 .3 Fluid core side . . . . . . . . . . . . . . . . . . . . 115

5.1 .4 Inner core . . . . . . . . . . . . . . . . . . . . . 117

5.2 Interface conditions . . . . . . . . . . . . . . . . . . . 118

5.2 .1 General case . . . . . . . . . . . . . . . . . . 118

5.2 .2 Mantle-side . . . . . . . . . . . . . . . . . . . . 120

5.2 .3 Inner core side . . . . . . . . . . . . . . . . . . . . . 122

5.2.4 Discussion on the solutions of $\widetilde{B}_{\varphi}$ at ICB and CMB . . . . . . . . 124

5.3 Electromagnetic torque models . . . . . . . . . . . . . . . . . 126

6 Elementary cases: validation of the electromagnetic torque models 129

6.1 System . . . . . . . . . . . . . . . . . . . . . . 130

6.2 Solid-body rotation . . . . . . . . . . . . . . . . . . . . 131

6.2.1 Case-study simulations . . . . . . . . . . . . . . . . . . . 131

6.2.2 Without gravitational coupling . . . . . . . . . . . . . . 132

6.2.3 With gravitational coupling . . . . . . . . . . . . 135

6.2.4 Expressions of $\mathcal{S}$ and $\mathcal{D} \ldots \ldots \ldots \ldots . \ldots \ldots$

6.3 Shear in the fluid outer core . . . . . . . . . . . . . . . . . . . . . . . 141

6.3.1 Shear flow . . . . . . . . . . . . . . . . . . . . 141

6.3.2 Expressions of $\mathcal{S}$ and $\mathcal{D} \ldots \ldots \ldots \ldots \ldots$. . . . . . . . . . 145

6.4 Conclusion . . . . . . . . . . . . . . . . . . . . . . . . . . 148

7 Amplitude of rotational fluctuations in geodynamo simulations $\quad 151$

7.1 Typical standard deviations . . . . . . . . . . . . . . . . . . . 152

7.2 Electromagnetic torques . . . . . . . . . . . . . . . 153

7.3 A first estimate . . . . . . . . . . . . . . . 156

8 Conclusions and perspectives $\quad 159$

A Appendices 161

A.1 Interface conditions . . . . . . . . . . . . . . . . . . . . 161

A.2 Time-dependent Electromagnetic torques . . . . . . . . . . . . . . 167

A.3 Elementary Cases . . . . . . . . . . . . . . . . . . . . . . . . . 171

$\begin{array}{lr}\text { List of Figures } & 177\end{array}$

$\begin{array}{ll}\text { Bibliography } & 177\end{array}$ 


\section{Introduction}

\subsection{Prologue}

\subsubsection{De l'aimant à la boussole}

En 77 après J.-C., Pline l'Ancien tente de réunir les connaissances et cultures de son temps et publie une encyclopédie de trente-sept volumes, Naturalis Historia. Dans le livre trente-six, Pline l'Ancien nous relate l'histoire d'un berger Grec du nom de Magnès qui, faisant paître ses troupeaux sur le mont Ida, ressentit que les clous de ses semelles étaient attirés par de la roche. Depuis lors, les phénomènes d'aimantations relèvent de ce qu'on appelle le magnétisme. Cette encyclopédie resta longtemps une référence dans de nombreux domaines et influença grandement les scientifiques du moyen-âge. C'est d'ailleurs à cette époque que l'on retrouve les premières traces de l'utilisation la plus connue du magnétisme : la boussole. La première utilisation de la boussole en tant qu'instrument d'orientation date du XI ${ }^{\mathrm{e}}$ siècle, sous la dynastie des Song (960-1279), en Chine. On retrouve cependant des systèmes tels que présentés figure 1.1 dès la dynastie des Han (206 av. J.-C. à 220 apr. J.-C.), même si l'utilisation de tels dispositifs pour l'orientation est difficile à prouver et reste débattue par les historiens.
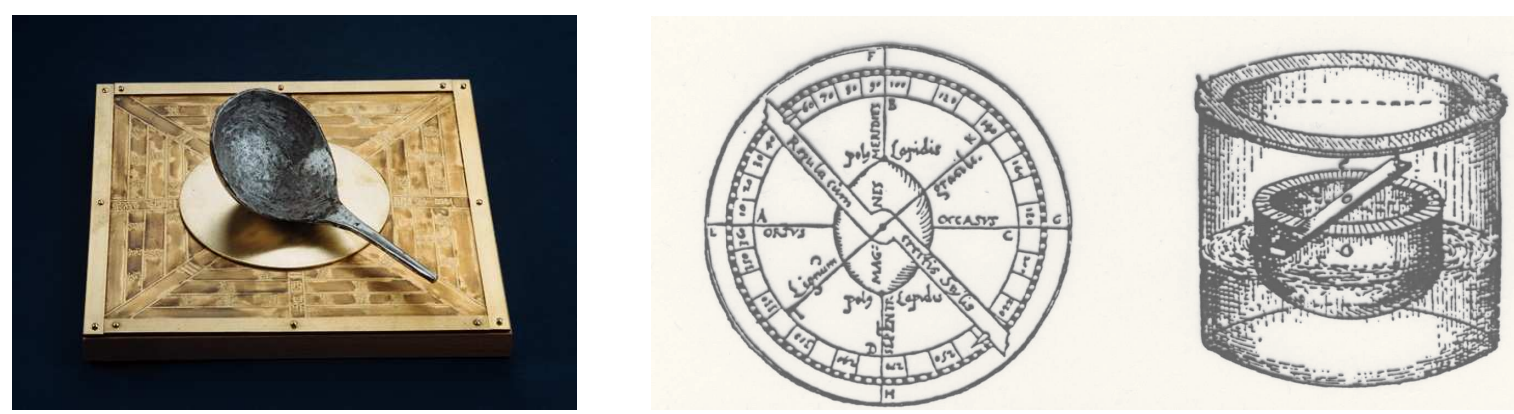

Figure 1.1 - Cuillère métallique pointant vers le Sud, découverte en Chine et datant de la dynastie des Han, à gauche. À droite, la boussole (marinette) du début du XIII ${ }^{\mathrm{e}}$ siècle en Europe.

En Europe, les premières références à une boussole, aussi appelée marinette, sont 
attribués aux moines Alexander Neckam, de l'abbaye de St Alban (1197) et Guiot de Provins, de l'abbaye de Cluny (1202), apportant la preuve de son utilisation pour la navigation (à droite figure 1.1). C'est cependant Pierre de Maricourt qui, dans son traité sur les propriétés des aimants Epistola de magnete, perfectionne le principe de la boussole. Il s'agit véritablement du premier texte scientifique relatif au magnétisme, dans lequel Pierre de Maricourt y décrit les propriétés d'attraction et de répulsion des pôles des aimants, ainsi que le fait qu'en cassant un aimant en deux morceaux nous obtenons nécessairement deux autres aimants, avec chacun deux pôles magnétiques. Ainsi, même s'il ne l'exprime pas directement, Pierre de Maricourt formula également l'hypothèse de non existence d'un monopôle magnétique.

\subsubsection{Premières observations}

Depuis lors, la boussole permit aux marins du monde entier de se repérer dans l'espace, en sachant toujours où se trouve le pôle Nord, ou plus exactement le pôle géomagnétique Nord. Cette précision, découverte au milieu $\mathrm{du} \mathrm{XV}^{\mathrm{e}}$ siècle, est cruciale pour les marins puisque ceuxci remarquèrent que la boussole, selon l'endroit où l'on se trouve, ne s'oriente pas exactement vers le pôle géographique Nord. L'angle ainsi formée entre les pôles géomagnétiques et géographiques est appelé déclinaison magnétique. La déclinaison magnétique est l'un des sujet d'étude de William Gilbert dans un livre intitulé De Magnete, à gauche figure 1.2, paru en 1600. Dans ce travail, considéré par certains comme l'un des premiers ouvrages scientifiques modernes, William Gilbert alors médecin de la reine, décrit le champ magnétique terrestre et en offre une vision similaire à celle que nous avons à l'heure actuelle qu'il appelle terrella, visible à droite figure 1.2. Cette terrella résulte d'expériences menées à l'aide d'une magnétite sphérique, assimilée à la Terre, ainsi que d'aiguilles de Fer. Elle met notamment en évidence une autre propriété importante du champ magnétique terrestre, découverte par Robert Norman (The Newe Attractive, 1581), l'inclinaison magnétique : l'angle entre une aiguille aimantée et le plan horizontal.

\subsubsection{Premières théories}

De par ses observations et ses expériences, William Gilbert écrit :

"Magnus magnet ipse est globus terrestris"

- W. Gilbert, 1600

Ainsi au début du XVII ${ }^{\mathrm{e}}$ siècle, au vu des similarités entre le champ magnétique terrestre et une magnétite sphérique, le champ magnétique terrestre est perçu et assimilé à celui d'un aimant situé au centre de la Terre. Cette théorie, bien qu'élégante, est cependant remise en 

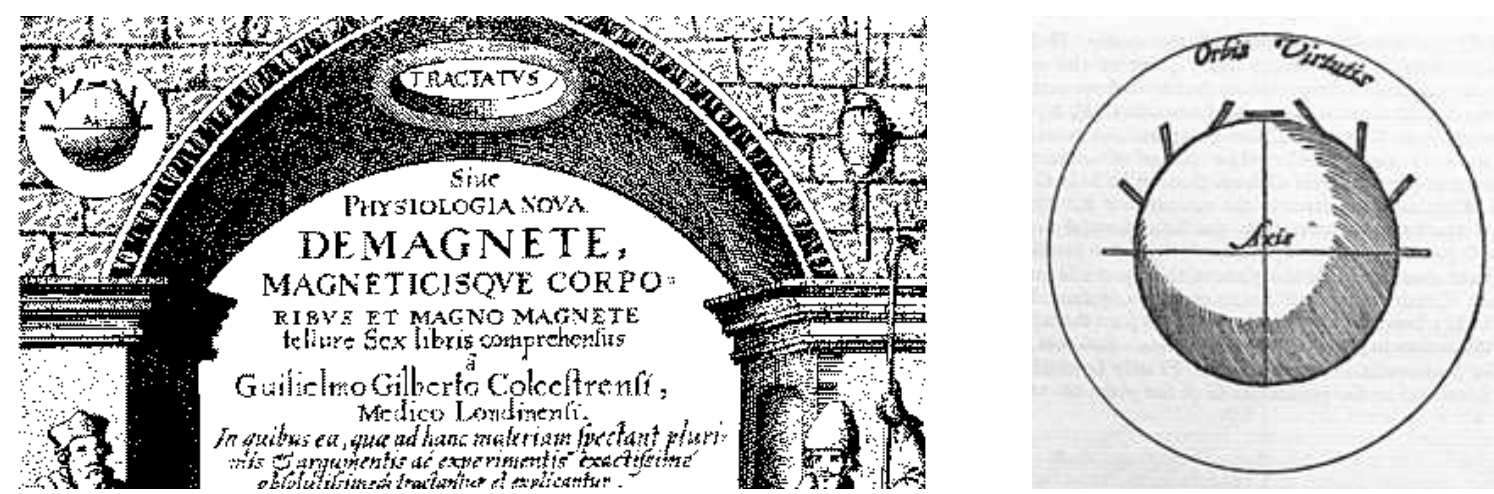

Figure 1.2 - À gauche, couverture de De Magnete, de William Gilbert, paru en 1600. Et à droite, le modèle de Terre comme un gigantesque aimant, la « Terrella» de William Gilbert.

cause trente-quatre ans plus tard par le mathématicien Anglais Henri Gellibrand. Basé sur plusieurs décennies de mesures, ce dernier mit en évidence la variabilité de la déclinaison magnétique au cours du temps. Cette variation, qualifiée de « séculaire », est contradictoire avec l'idée d'un aimant fixe proposé par Gilbert et donna suite à différentes théories. La plus célèbre fut développée par Edmund Halley en 1692, connu pour avoir calculé la périodicité de la comète qui porte son nom. Dans son article «On the cause of the change in the variation of the magnetic needle, with a hypothesis of the structure of the internal parts of the Earth », publié dans la revue scientifique Philosophical transactions of the Royal Society of London, Edmund Halley réunit tout d'abord toutes les mesures de déclinaison magnétique disponibles à l'époque. De ces données, il observe qu'en de nombreux endroits sur Terre la déclinaison magnétique est de plus en plus vers l'ouest au cours du temps. Il décrit ainsi pour la première fois une des caractéristiques principale de la variation séculaire du champ magnétique terrestre : la dérive géomagnétique vers l'ouest (voir carte figure 1.3). Pour l'expliquer, Edmund Halley formule une théorie basée sur des sphères concentriques, séparées par du vide, portant chacune une partie du champ magnétique terrestre et qui serait en rotation différentielle (schéma figure 1.3). Dans son modèle le plus simple, à deux sphères, cette théorie implique une dérive vers l'ouest de la déclinaison magnétique.

\subsubsection{Intérieur de la Terre}

La théorie d'Edmund Halley vise à expliquer les variations observées du champ magnétiques terrestre mais il propose également une idée de la structure interne de la Terre. Parmi les plus illustres théories, on notera celle d'Aristote, qui décrit une Terre fixe, au centre de l'univers, composée des quatre éléments : air, terre, eau et feu. Cette vision restera d'ailleurs jusqu'à ce que Copernic, en 1543, prouve que la Terre, à l'image des autres planètes, tourne autour du Soleil. René Descartes, en 1644, décrit une Terre composée d'une croûte rocheuse reposant sur une mer intérieure, ce qui permettait selon lui d'expliquer les différents reliefs de la surface terrestre. Un siècle plus tard, à l'image d'Athanaisus Kircher (« Mundus 

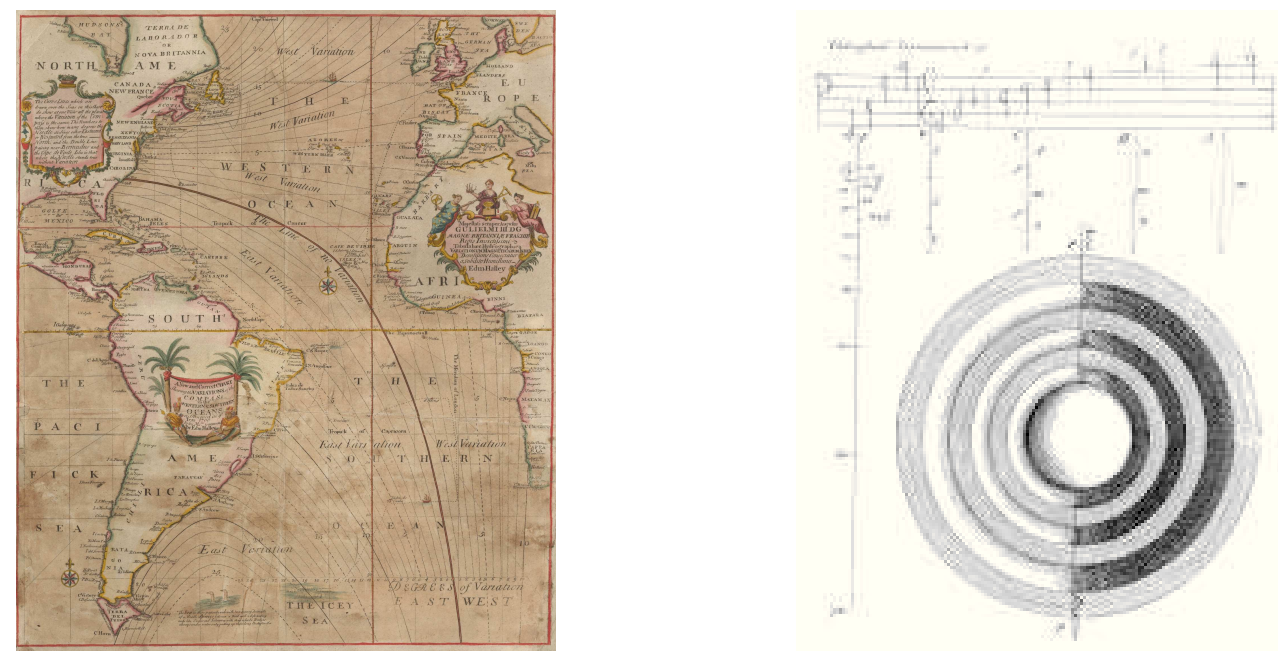

Figure 1.3 - À gauche, une carte de la dérive de la déclinaison magnétique de E. Halley. À droite, un schéma extrait de Halley (1692) illustrant la théorie des sphères magnétique concentriques en rotation différentielle.

subterraneus », 1665), Georges Louis Buffon imagine la Terre comme un ancien morceau de Soleil, expulsé par le passage d'une comète, refroidi et à présent homogène (« Théorie de la Terre », 1749). Les premières réponses à de telles questions furent obtenues suite aux progrès de la tomographie sismique. Cette branche de la sismologie étudie la trajectoire et la vitesses des ondes sismiques qui se propagent à l'intérieur de la Terre à la suite d'un séisme. La vitesse des ondes sismique étant proportionnelle à la composition des matériaux, la température et la pression, l'étude de la propagation de ces ondes permet donc en théorie d'obtenir la composition de l'intérieur de la Terre. C'est Edouard Roche qui, s'inspirant de la structure des météorites, proposa le premier modèle différencié de l'intérieur de la Terre. Ce modèle, publié en 1881, décrit ainsi la Terre comme composée de deux couches : une partie rocheuse et un noyau central composé essentiellement de Fer. Il fut ensuite appuyé par les travaux du chercheur Allemand Emil Wiechert, qui proposa un modèle semblable en 1897. Quelques années plus tard, Richard Dixon Oldham (1906) observe les premières ondes sismiques ayant traversées le noyau de la Terre, prouvant de ce fait son existence. En 1912, Beno Gutenberg détermina la profondeur à laquelle est situé le noyau, soit à 2900 kilomètres sous la surface. La nature liquide de ce noyau est mise en évidence par Harold Jeffreys en 1926. Enfin, c'est Inge Lehman, chercheuse Danoise, en 1936, qui découvrit la présence du noyau interne, solide, également appelé la graine.

La Terre est aujourd'hui représentée, dans son modèle le plus simple, comme une sphère de 6370 kilomètres de rayon et composée de quatre couches (voir figure 1.4). La première est la croûte, qui représente une épaisseur moyenne de trente kilomètres aux niveaux des continents (composition granitique) et de dix kilomètres pour les océans (composition basaltique). La seconde couche, bien plus conséquente, est appelée le manteau. Elle représente environ 2800 kilomètres d'épaisseur et est principalement composée de péridotite. Le noyau est quant à lui composé essentiellement de Fer (85\%), de Nickel (10\%) 


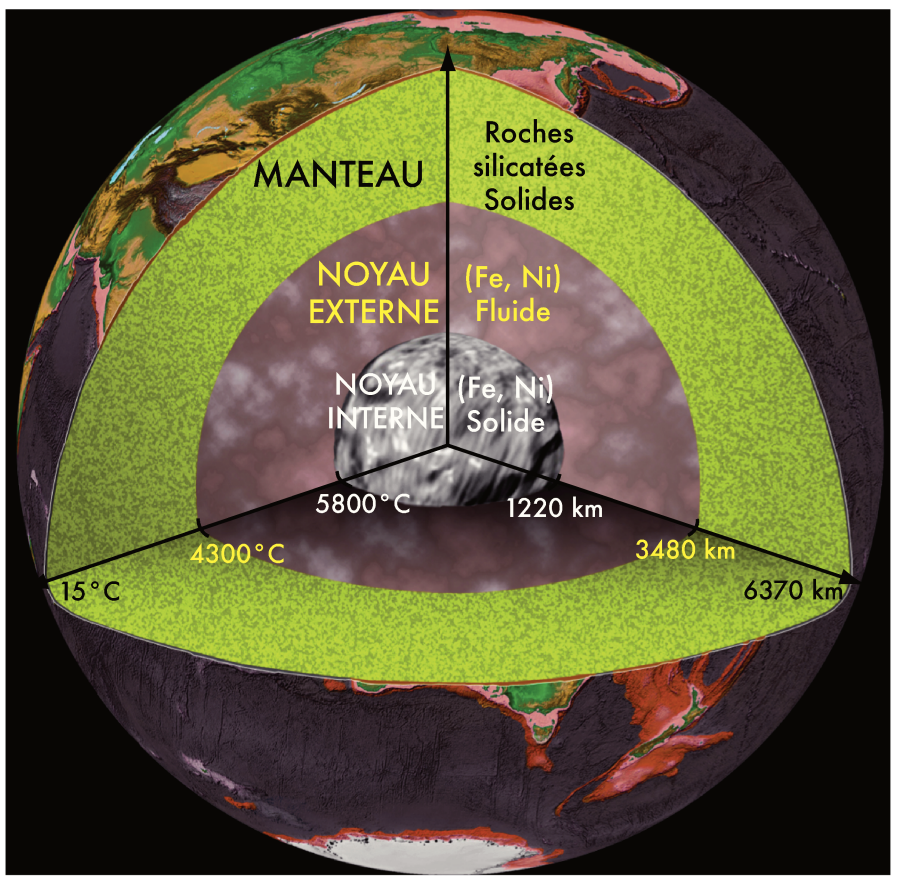

Figure 1.4 - Schéma de l'intérieur de la Terre, avec la participation de l'équipe de Géosciences du Palais de la Découverte.

et d'autres éléments plus légers tel que le Soufre, le Silicium ou encore l'Oxygène. On distingue deux parties, le noyau externe liquide et le noyau interne solide.

\subsubsection{La géodynamo}

Parallèlement aux nombreuses découvertes sur la structure interne du globe, Hale (1908) démontre l'existence du champ magnétique solaire par l'observation et la mesure des taches solaires. Quelques années plus tard, Joseph Larmor formula trois hypothèses différentes pour expliquer l'origine du champ magnétique du Soleil. Parmi ces trois hypothèses, il n'en retient cependant qu'une seule, qui serait selon lui applicable au champ magnétique terrestre, à condition que la Terre possède en son centre une partie fluide (Larmor, 1919). Il propose que le champ magnétique solaire, et donc possiblement celui de la Terre, soit le résultat d'une dynamo auto-entretenue. Pour le Soleil, cela correspond à affirmer que les mouvements du plasma engendrent, en interagissant avec un champ magnétique pré-existant, des courants électriques qui sont à leur tour susceptibles de générer un champ magnétique. Ce mécanisme de dynamo est nommé « auto-entrenu » car il permet d'entretenir un champ magnétique de grande échelle à partir de mouvements de fluide conducteur d'électricité. Le premier modèle de géodynamo est ainsi proposé par Walter Elsasser en 1946. Celui-ci reprend l'idée émise par Joseph Larmor et la théorise en déterminant le moteur des mouvements du Fer liquide à l'intérieur du noyau : la convection. La convection est un mode de transfert de chaleur par déplacement de la matière. Autrement dit, si une partie d'un fluide est plus chaude (et par conséquent moins dense) que le milieu environnant, celle-ci sera entrainée vers le 

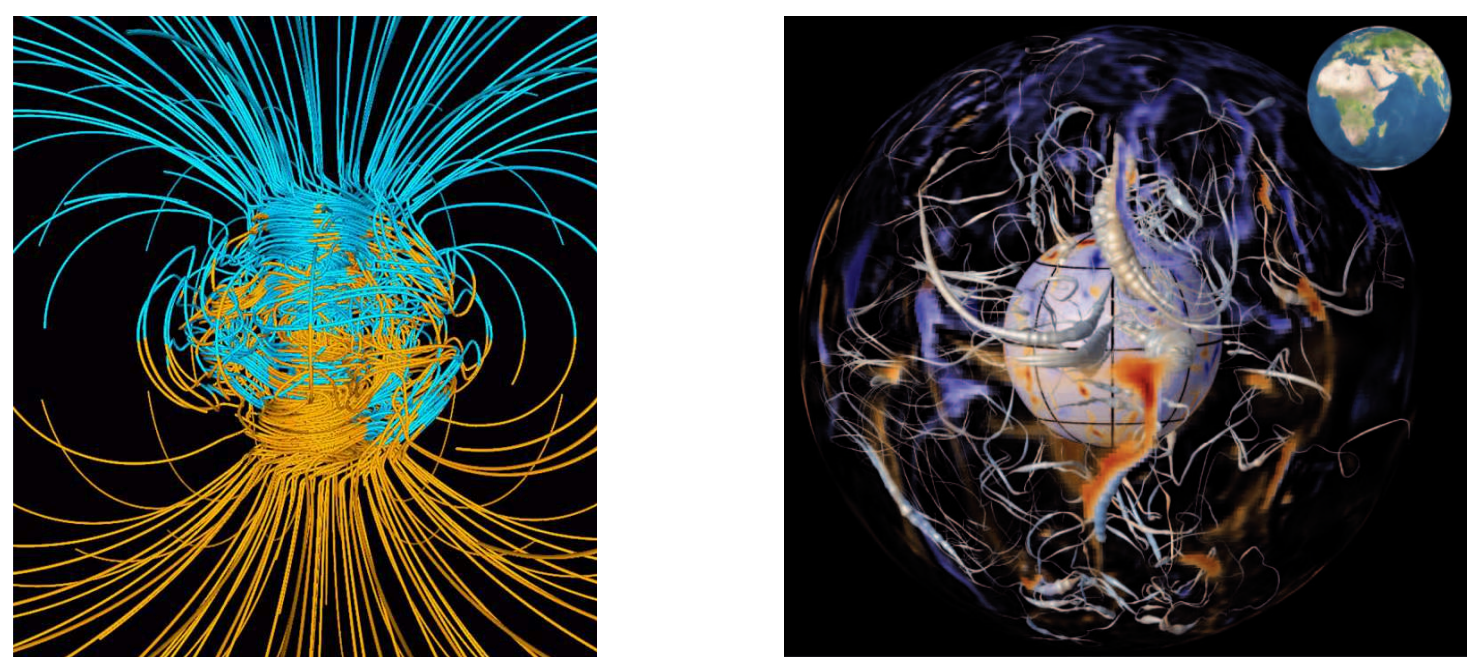

Figure 1.5 - Simulations numériques de Glatzmaier and Roberts (1995) à gauche, et Aubert et al. (2013) à droite.

haut sous l'effet de la force d'Archimède. A l'intérieur du noyau fluide, cette convection dite thermique est naturellement engendrée par la différence de températures entre la frontière noyau-manteau et la frontière graine-noyau externe (voir figure 1.4). Cependant, le moteur principal $(\sim 80 \%)$ des mouvements de convection est d'origine chimique. Le refroidissement lent et global de l'intérieur de la Terre, et ce depuis sa création, entraine en effet la cristallisation progressive de la graine. Cette cristallisation, qui de surcroit dégage de la chaleur, concerne uniquement le Fer et le Nickel, ce qui provoque nécessairement une importante émission des éléments légers constituants le noyau, à la surface de la graine. La combinaison des effets thermiques et chimiques entraine ainsi une convection turbulente du Fer liquide dans le noyau externe. Le Fer étant un matériel conducteur d'électricité ces mouvements produisent des courants électriques par interaction avec le champ magnétique, qui à l'heure tour amplifient le champ magnétique : c'est la géodynamo.

\subsubsection{Simulations numériques}

L'un des outils actuels pour étudier la géodynamo est de résoudre les équations de la MHD (magnétohydrodynamique) à l'aide de super-ordinateurs et ainsi tenter de reproduire le champ magnétique terrestre en simulant les possibles écoulements du fer liquide dans le noyau. La première simulation numérique reproduisant les principales caractéristiques du champ magnétique terrestre de façon auto-entretenue fut réalisée par Gary Glatzmaier et Paul Roberts en 1995. La figure 1.5 (à gauche), qui est un état de la simulation numérique à un instant donné, montre alors la complexité des lignes de champ magnétiques à l'intérieur du noyau, ainsi que la forme du champ magnétique de grande échelle qui en résulte (Glatzmaier and Roberts, 1995). Outre le progrès majeur concernant la modélisation du champ magnétique terrestre, Gary Glatzmaier et Paul Roberts remarquèrent que dans leur simulation la graine était en rotation différentielle vers l'Est par rapport au manteau 
d'environ $2^{\circ}$ par an (Glatzmaier and Roberts, 1996). Ce phénomène, directement lié à l'intensité du champ magnétique et aux mouvement du fer liquide, est appelé super-rotation de la graine. Cette observation dans les simulations numériques donna suite à de nombreuses études sismiques tentant de mesurer cette possible rotation différentielle de la graine, dont une la même année par Song and Richards (1996). Cependant, il est à l'heure actuelle encore difficile d'obtenir des informations aussi précises sur la dynamique d'un object situé au centre de la Terre, et cette propriété de la Terre interne reste mal contrainte.

\subsubsection{Progrès et objectif de la thèse}

À droite de la figure 1.5 est représenté le résultat d'une autre simulation numérique de la géodynamo, qui pour la première fois réussi à reproduire les caractéristiques principales de la dérive vers l'ouest du champ magnétique terrestre (Aubert et al., 2013). Etant observée depuis les premières mesures du champ magnétique terrestre et représentant la majeure partie de sa variation séculaire, la reproduction de la dérive géomagnétique vers l'ouest dans les simulations numériques est effectivement un progrès majeur pour le géomagnétisme. Pour arriver à ce résultat, cette simulation numérique considère les différents couples qui relient le manteau, le noyau fluide et la graine, et modélise une croissance hétérogène de cette dernière. Ces considérations ont pour conséquence de produire un écoulement principalement vers l'ouest, proche de la frontière noyau-manteau, et de localiser cet écoulement sous la zone Atlantique, correspondant aux valeurs de dérive vers l'ouest du champ magnétique mesurées en surface.

L'objectif de cette thèse est d'utiliser ces simulations numériques récentes reproduisant les caractéristiques fondamentales du champ magnétique terrestre, telles qu'observées depuis quatre siècles, afin de pouvoir contraindre la super-rotation de la graine. Pour cela, il est tout d'abord nécessaire de formuler et de valider dans les simulations numériques des modèles des couples qui relient et gouvernent la rotation du manteau, du noyau fluide et de la graine. Une fois ces modèles établis, ils permettent de faire le lien entre la dérive géomagnétique vers l'ouest et la super-rotation de la graine. 


\subsection{Earth's core structure and dynamics}

\subsubsection{Internal structure of the Earth}

The first geodynamo theories developed after the substantial progress of seismology in providing insights on the Earth's core. In 1897, Emil Wiechert first suggested the existence of a metallic core. A few years later, Oldham (1906) first detected the presence of the core and Gutenberg (1913) determined the depth of the core-mantle boundary (CMB) as being close to $2900 \mathrm{~km}$. The distinction between the outer and inner core is however attributable to the works of Jeffreys (1926), who showed the liquid state of the core, and Lehmann (1936) who discovered the solid inner core. These pioneering works gave rise to numerous seismological studies of the Earth's interior, comprising $\mathrm{P}$ and $\mathrm{S}$ waves travel times as well as normal modes, that were later exploited by Dziewonski and Anderson (1981) to develop the Preliminary Reference Earth Model (PREM). As being a 1D model, the main assumption of the PREM model is that the Earth is radially homogeneous. It establishes the velocity of $\mathrm{P}$ and $\mathrm{S}$ waves and the density of Earth's materials as a function of depth. The original figure of the PREM model is displayed in figure 1.6. It exhibits the liquid state of the outer core by the non-propagation of the $\mathrm{S}$ waves from the CMB to the inner core boundary (ICB). The density jump at the CMB clearly points the difference in composition between the mantle (silicates) and the core (metallic), while the density jump at the ICB reflects the phase transition of iron from liquid to solid. Though the PREM model defines the variation of density with depth inside the Earth, it does not determine the composition of the materials. The metallic composition of the core was inferred by Birch (1952) as being a Fe-Ni alloy, by compressional experiments on alkali metals. Birch (1952) also suggested the presence of lighter elements within the outer core, while the inner core should be mostly crystallized iron. The solid state of the inner core was later confirmed by Jacobs (1953) who showed that, given the temperatures and pressures inside the core, there should be a phase transition of iron from liquid to solid close to the inner core boundary.

In order to assess the exact composition of the core, it is however necessary to refer to geochemical arguments. The main idea behind these arguments is to suppose that the Earth is a differentiated meteorite, so that its bulk composition should ressemble that of meteoritic materials. Then, mostly by using ratios between major and trace elements, and considering the lithophile or siderophile character of the elements, it is possible to infer the composition of the silicate Earth (crust and mantle) and the core. However, with this approach, the presumed compositions are naturally sensitive to both the choice of meteoritic parent body and the considered model of Earth's formation. Table 1.1 exposes two well-known models, the first one was developed by Allègre et al. (1995) and is based on carbonaceous chondrites, while the second one is based on enstatite chondrites (Javoy et al., 2010). 


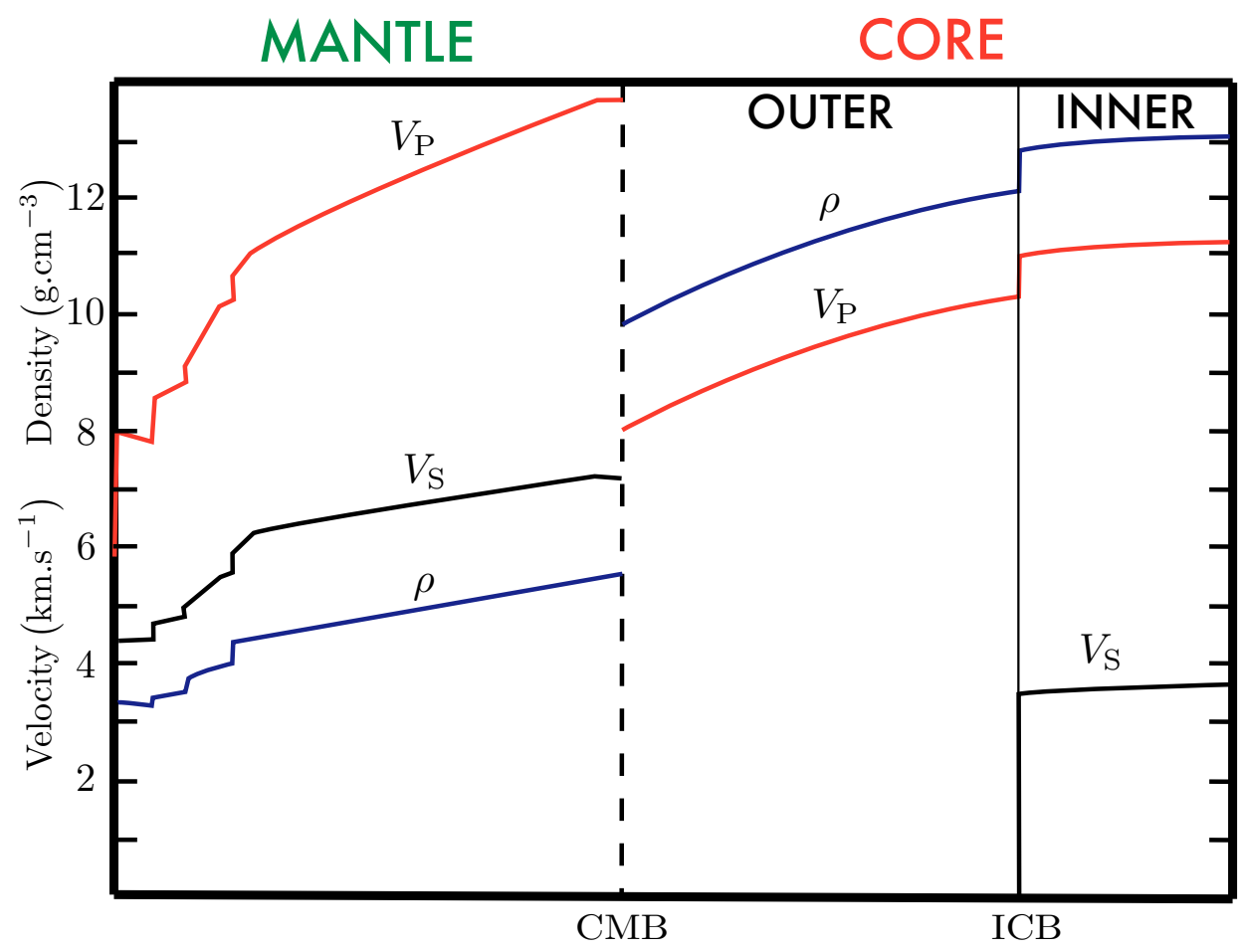

Figure 1.6: PREM model from Dziewonski and Anderson (1981), variation of the density $\rho$ (in blue), and the velocities of $\mathrm{P}$ and $\mathrm{S}$ waves (red and black), as functions of depth.

\begin{tabular}{l|c|c}
\hline \hline & \multicolumn{2}{c}{ Composition of the core in wt\% } \\
\hline Elements & Allègre et al. (1995) & Javoy et al. (2010) \\
\hline \hline $\mathrm{Fe}$ & $79.39 \pm 2.00$ & $85.50 \pm 1.14$ \\
$\mathrm{Ni}$ & $4.87 \pm 0.30$ & $5.35 \pm 0.80$ \\
$\mathrm{Si}$ & 7.35 & $6.64 \pm 0.51$ \\
$\mathrm{O}$ & $4.10 \pm 0.50$ & $1.99 \pm 0.46$ \\
$\mathrm{~S}$ & $2.30 \pm 0.20$ & - \\
$\mathrm{Cr}$ & $7.8 \times 10^{-3}$ & $0.55 \pm 0.05$ \\
$\mathrm{Co}$ & $2.5 \times 10^{-3}$ & $0.25 \pm 0.03$ \\
\hline \hline
\end{tabular}

Table 1.1: Composition of the core in weight percent (wt\%) in Iron (Fe), Nickel (Ni), Silicium $(\mathrm{Si})$, Oxygen (O), Sulfur (S), Chrome (Cr) and Cobalt (Co), from Allègre et al. (1995) and Javoy et al. (2010).

\subsubsection{Dynamics of the core}

The geodynamo theory began with the idea of Larmor (1919) concerning the magnetic field of the Sun. He proposed that the Sun's magnetic field is produced by the motion of electrically conducting plasma, producting electric currents that would in turn increase and maintain the preexisting magnetic field. He named this mechanism a self-excited dynamo, and assumed that, if the Earth's core is fluid, then this mechanism "would account for magnetic change, sudden or gradual, on the earth...". This idea was further refined by Elsasser (1946a) who developed a model for the Earth's dynamo, capable of maintaining 
a large-scale magnetic field, and explaining its secular variation (Elsasser, 1946b). This model, based on the balance between the Coriolis and Lorentz forces, was further improved in the first kinematic dynamo model of Bullard and Gellman (1954), consisting in finding velocity fields of electrically conducting fluids that are able to produce and maintain large-scale field. However, prescribing a velocity field and obtaining a large-scale magnetic field is only one part of the problem, which global answer lies in the MHD equations (Magnetohydrodynamics), the conservation of mass, momentum and energy, defining the overall dynamics of the core.

The dynamics of the core is mainly driven by convection. As the whole Earth since its formation, the core is loosing heat at the rate that is controlled by the core-mantle boundary heat flux, thus inducing a secular cooling that can promote convection. However, this secular cooling a also a source of buoyancy forces, through the freezing of the inner core. Indeed, Braginsky (1963) concluded that the density jump at the ICB (see figure 1.6) is not only due to contraction on freezing, but also reflects the fact that the cristallisation mostly involves iron. The freezing of iron at the ICB is this a source of chemical buoyancy, by the release of light elements, and thermal buoyancy, through the latent heat release of the phase change of iron. Note that such a driving force may only account for the last hundreds of millions of years since the inner core is assumed to be 1 billion year of age (Labrosse et al., 2001) or even less Labrosse (2015), while Tarduno et al. (2015) found that the Earth had a magnetic field 4.2 billions years ago. Still, as the viscosity of iron at the core conditions is close to that of water at the surface (Poirier, 1988), the buoyancy release at the ICB is the source of highly turbulent convection in the fluid outer core. This convection is organized in columnar structures, as anticipated by Roberts (1968) and Busse (1970) and experimentally showed by Carrigan and Busse (1983). This particular flow arrangement into Taylor columns is a direct consequence of the Proudman-Taylor theorem, that predicts geostrophic flows (invariant along the axis of rotation) in the case where the Coriolis force dominantes the force balance of the system. These mainly helical flows are able to produce strong poloidal magnetic field through alpha-effect, as described by Parker (1955). The resulting magnetic field of such a configuration is mainly dipolar, as observed for the Earth's field.

\subsection{Earth's magnetic field}

The large-scale magnetic field may be associated to a dipole field which intensity $(\mathrm{F})$ is around $30000 \mathrm{nT}^{1}$ close to the equator and $60000 \mathrm{nT}$ at higher latitudes. The magnetic field vector, $\mathbf{B}$ can be described on the orthgonal basis $\left(\mathbf{e}_{\mathbf{x}}, \mathbf{e}_{\mathbf{y}}, \mathbf{e}_{\mathbf{z}}\right)$ by $\mathbf{B}(X, Y, Z)$, with $\mathbf{e}_{\mathbf{x}}$ pointing towards the geographic North, $\mathbf{e}_{\mathbf{y}}$ towards the East and $\mathbf{e}_{\mathbf{z}}$ downward. However, it is often defined by its intensity $F=\sqrt{X^{2}+Y^{2}+Z^{2}}$ and two angles like $\mathbf{B}(F, D, I)$. The first one is the declination $D$, the angle between the geographic North and the geomagnetic

1. for nanoTesla, $1 \mathrm{nT}=10^{-9} \mathrm{~T}$. 


$$
\begin{gathered}
\mathbf{B}=X \cdot \mathbf{e}_{\mathbf{x}}+Y \cdot \mathbf{e}_{\mathbf{y}}+Z \cdot \mathbf{e}_{\mathbf{z}} \\
D=\arcsin \frac{Y}{\sqrt{X^{2}+Y^{2}}} \\
I=\arctan \frac{Z}{\sqrt{X^{2}+Y^{2}}}
\end{gathered}
$$

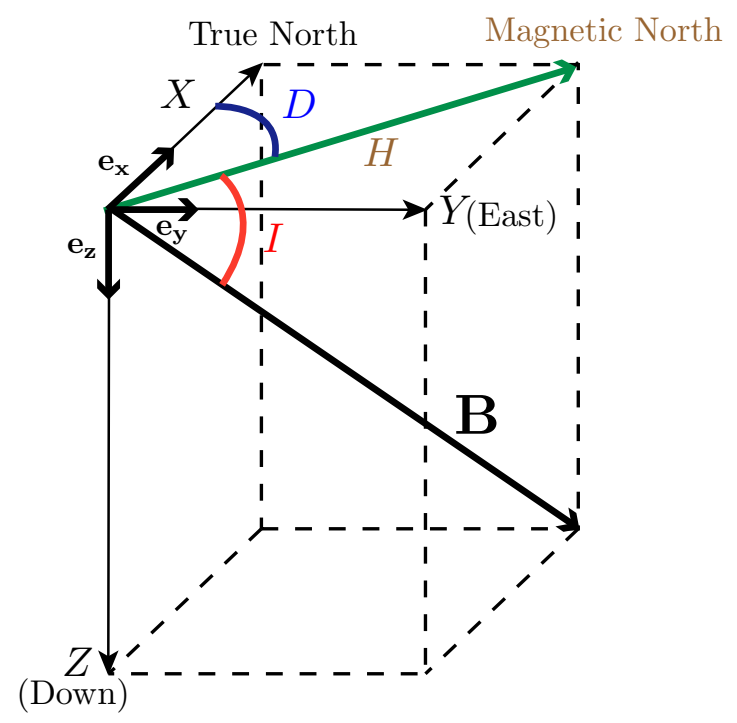

Figure 1.7: Cartesian coordinates system used to describe the magnetic field vector $\mathbf{B}$. $\mathbf{H}$ is the projection of $\mathbf{B}$ in the horizontal plane, pointing towards the magnetic North pole. $D$ is the magnetic declination, the angle between the geographic and the magnetic North (between the $\mathbf{e}_{\mathbf{x}}$ component of $\mathbf{B}$ and $\mathbf{H})$. I is the magnetic inclination, the angle between the horizontal plane and the magnetic field vector $\mathbf{B}$.

North in the horizontal plane, while the second is the inclinaison $I$, the angle between the horizontal plane and the downward component of the magnetic field. The geometry and the relations between the different components are displayed in figure 1.7.

\subsubsection{Geomagnetic observations}

"Only here I must take leave to recommend to all masters of ships that they use they utmost diligence to make, or procure to be made, observations of these variations in all parts of the world..."

- Halley (1692)

In order to understand the Earth's magnetic field, we now have about four centuries of observations. These observations comprise the declination measurements made by mariners since the discovery that the geographic and magnetic north poles of the Earth do not perfectly coincide, in the middle of the 17th century. These observations offer a wide coverage of the Earth, but are mainly concentrated in trade routes (see figure 1.8). The more accurate data on the magnetic field are obtained in geomagnetic observatories. The very first geomagnetic observatory was located in the garden of the Paris observatory, dedicated to Astronomy. Its construction was ordered by François Arago in 1823 who measured the three components of the magnetic field vector: declination, inclination and intensity. However, rigorous measurements of the magnetic field intensity were firstly obtained by Carl Friedrich 


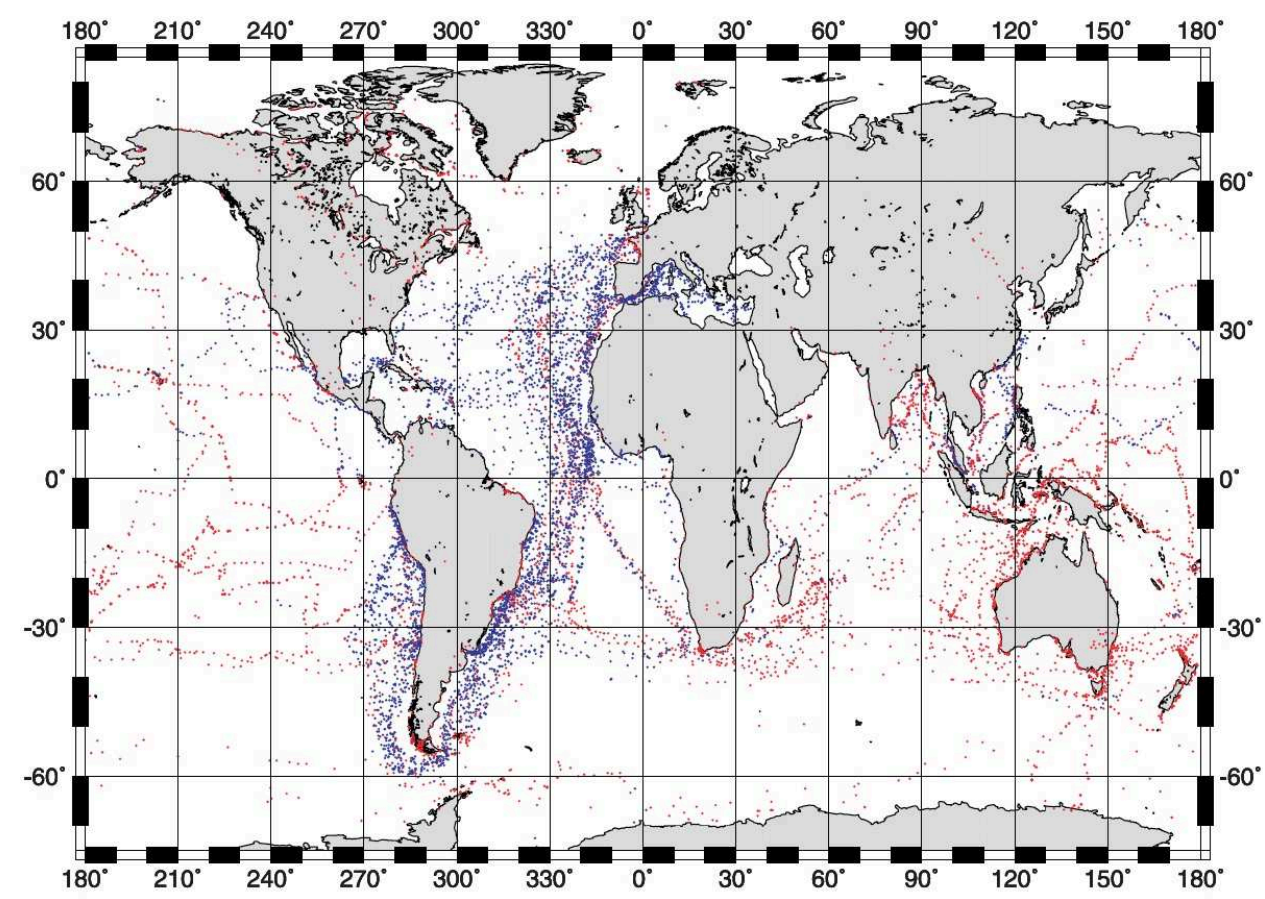

Figure 1.8: Global distribution of all declination measurements found in the Archives Nationales document. Red dots represent observations made by ships in the Service Hydrographique; blue dots represent the other French naval measurements. Note in particular the coverage in the Pacific and along South American shores. From Jackson et al. (2003).

Gauss in the city of Göttingen in 1833, trough the development of new instrumentation and an electrostatic and electromagnetic unit system. Nowadays, a great part of the magnetic observatories in the world (over 108) form the INTERMAGNET ${ }^{2}$ network, a non-exclusive programme of magnetic data exchange. Finally, although a relatively wide coverage may be obtained by boarding magnetometers in ships or planes, the preferred way to gather global observations on the Earth's magnetic field requires satellites. The history of magnetic satellites began with the POGO and OGO satellites, between 1965 and 1971, that provided a global coverage the magnetic field intensity. Then Magsat (1989-1980), which was the first equipped with a vector magnetometer, helped to determine the three components of the vector field. Since then, several satellite missions brought more and more informations on the Earth's field: Ørsted, (1999-present), CHAMP (2000-2010) and SAC-C (2001-2004). The most recent satellite mission, called Swarm (see Friis-Christensen et al., 2006), was launched in November 2013 and is composed of three satellites, one in polar orbit around $510 \mathrm{~km}$ of altitude and a tandem of satellite in polar orbit around $450 \mathrm{~km}$ of altitude. This configuration was designed in order to acquire very accurate data on the intensity and the components of the magnetic field vector, in addition to bring informations on the different sources of magnetic field close to the Earth's surface.

2. See www.intermagnet.org for more informations 

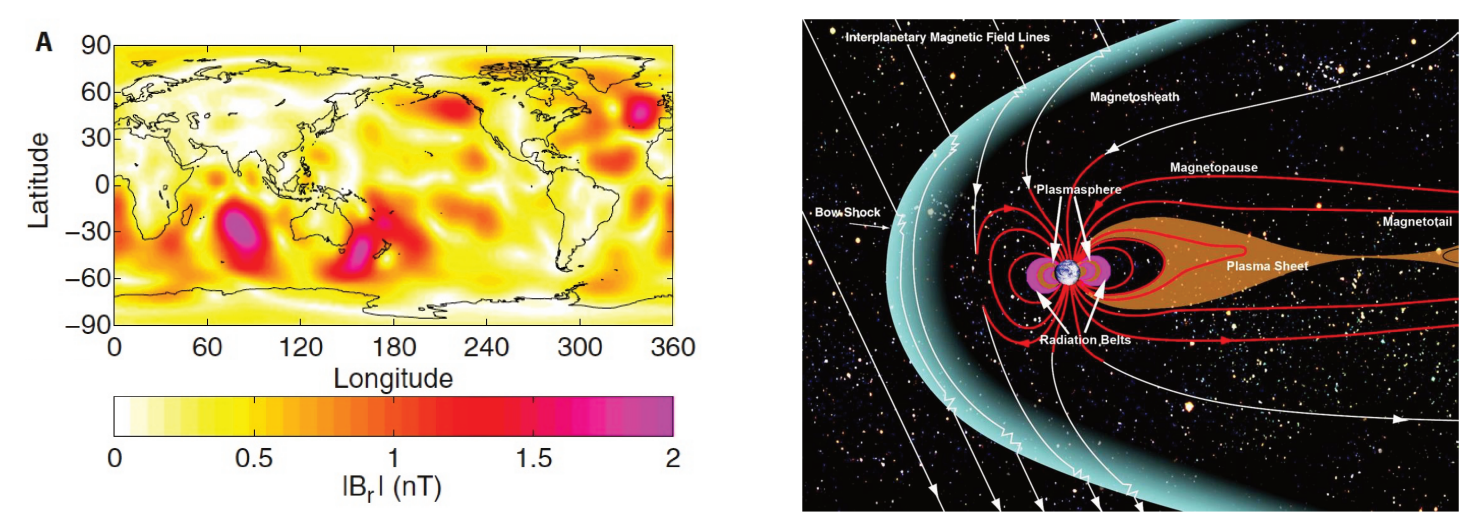

Figure 1.9: On the left, the amplitude of the radial magnetic field component due to the $M_{2}$ oceanic tides at an altitude of $430 \mathrm{~km}$, extracted from satellite data, from Grayver et al. (2016). On the right, a schematic of the magnetosphere from www.nasa.gov.

\subsubsection{Other sources}

As observed on the Earth's surface, the geomagnetic field is the result of internal and external sources. Indeed, superimposed on the mainly dipolar field coming from the core are several features that do not directly depend on the geodynamo process.

\section{External sources}

The external sources are produced in the magnetosphere, the limit beyond which the solar magnetic field dominates the Earth's field, and in the ionosphere, the highly ionized part of the Earth's atmosphere located between $60 \mathrm{~km}$ and $1000 \mathrm{~km}$ in elevation. In these regions, the solars winds and radiations are both substantial sources of electric currents that affects the Earth's field. As highly related to the Sun's activity, the external sources influence the Earth's magnetic field from hourly periods, related to solar storms, solar rotation (27 days), Earth's orbit (1 year), to decadal periods that are associated with solar cycles (11 years). The impacts of the external sources on the magnetic field (few hundreds of $\mathrm{nT}$ ) include the distortion of the magnetosphere due to solar winds (see figure 1.9), the diurnal variation in magnetic intensity due to solar radiation, the Equatorial Electrojet (a strong eastward ionospheric current in the day time equatorial regions), as well as the very observation of the polar auroras, that are the result of deviated solar particules interacting with the upper layer of the atmosphere.

\section{Internal sources}

The internal sources of magnetic fields, other than the geodynamo, are a combination of the remanent magnetization of crustal rocks and the induced internal field in both lithosphere and mantle. The first source is a historical record of the main field acquired by rocks during their formation and represent local anomalies on the order of hundreds of nT. Above the Curie temperature, iron-bearing minerals, such as magnetite of hematite, align 
in the direction of the main field. As the rock cools down, the orientation and the intensity of the main field are then partly trapped in the rock, in a process called thermal remanent magnetization. The second source is a magnetic field induced by the interaction between the main field and electric currents coming from the core or from external sources. Among the external currents are those produced by the oceanic tides, that also represent a small source of magnetic field (a few nT). Indeed, as a flow of an electrically conducting fluid, the ocean lunar semidiurnal tides, referred as $M_{2}$, are also a source of magnetic field (Tyler et al., 2003). Besides, the electric currents produced by this particular oceanic circulation may be useful to infer the electrical conductivity of lithosphere and mantle materials (Schnepf et al., 2015; Grayver et al., 2016).

\subsubsection{Magnetic field models}

As mentioned above, the external sources of magnetic field represent mostly short period signal in the temporal variation of the magnetic field. Moreover, as their intensity is small compared to the main field, geomagnetic studies on the main field assume that they are negligible. Considering the weak electrical conductivity of the rocks composing the lithosphere and the mantle, it is also conceivable to assume that current flows have no substantial influence on the main field observed at the Earth's surface. In such a situation, the magnetic field $\mathbf{B}$ at the Earth's surface can be described by a potential field like

$$
\mathbf{B}=-\nabla V
$$

where $V$ is the geopotential that must satisfy Laplace equation

$$
\Delta V=0
$$

For a perfectly insulating mantle, the solution can be expressed using a spherical harmonic (SH) decomposition (see e.g. Langel, 1987) and holds from the radius of the core $\left(r_{\mathrm{o}}\right)$ to the Earth's surface (r=a),

$$
V(r, \theta, \phi)=a \sum_{\ell=1}^{\infty} \sum_{m=0}^{\ell}\left(\frac{a}{r}\right)^{\ell+1}\left[g_{\ell}^{m} \cos m \phi+h_{\ell}^{m} \sin m \phi\right] \mathrm{P}_{\ell}^{m} \cos \theta
$$

with $g_{\ell}^{m}$ and $h_{\ell}^{m}$ the Gauss coefficients, expressed in the same units as the magnetic field, $\ell$ and $m$ the degree and the order of the spherical harmonic expansion and $\mathrm{P}_{\ell}^{m}$ the Schmidt quasi-normalized associated Legendre functions. In such a representation (see figure 1.11), the degree $\ell=1$ represents the dipole (axial for $m=0$ and equatorial for $m=1$ ) and higher degrees are associated with the quadrupole $(\ell=2)$ and the octupole $(\ell=3)$. Spherical harmonics for which $m=0$ are called zonal, while harmonics $\ell=m$ are referred to as sectorial. This description thus offers the spatial distribution of the magnetic field, which is 


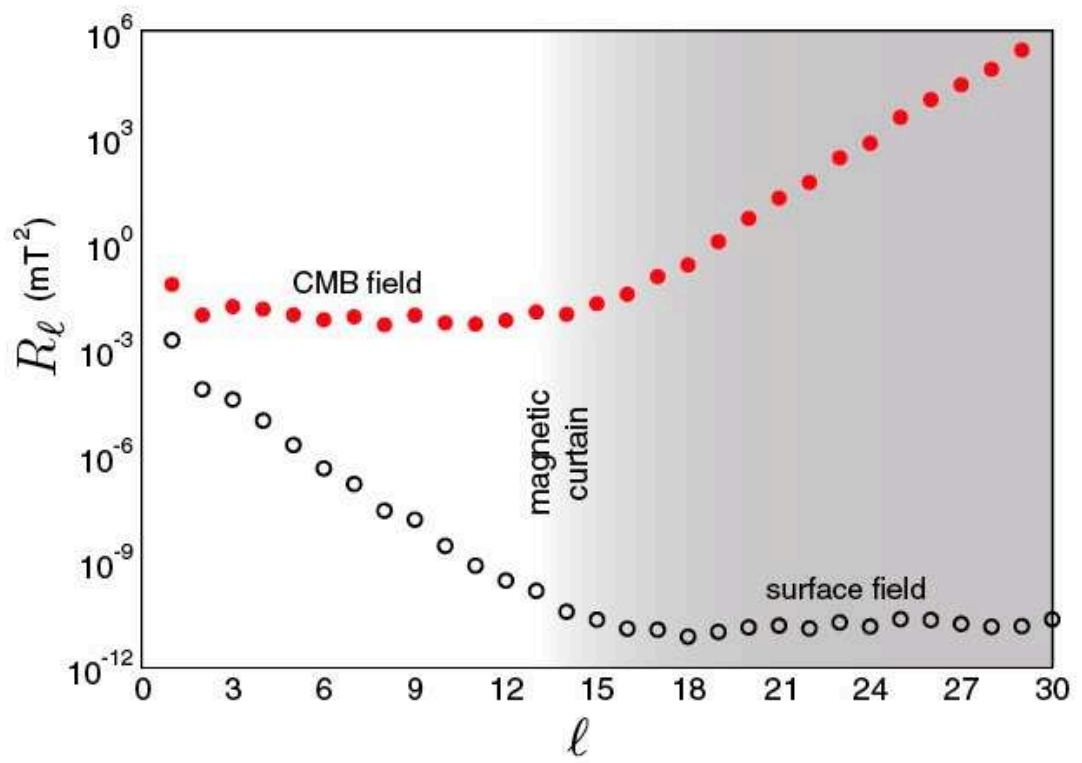

Figure 1.10: Mauersberger-Lowes spectrum of magnetic intensity of the xCHAOS model (Olsen and Mandea, 2008), from Roberts and King (2013). Hollow symbols stand for the spectrum at the surface $(r=a)$ while solid ones stand for the spectrum at the CMB $\left(r=r_{\mathrm{o}}\right)$.

also analyzed in term of power spectrum like

$$
R_{\ell}(r)=(\ell+1)\left(\frac{a}{r}\right)^{2 \ell+4} \sum_{m=0}^{\ell}\left[\left(g_{\ell}^{m}\right)^{2}+\left(h_{\ell}^{m}\right)^{2}\right]
$$

that defines the power of the magnetic field contained in each degree $\ell$ of the spherical harmonic expansion. At the Earth's surface, the graphic representations of $R_{\ell}=f(\ell)$ is referred to as a Mauersberger-Lowes power spectrum (Lowes, 1974). They generally exhibit a substantial increase in the power spectra for SH degrees greater than 13, visible in figure 1.10 that indicates the influence of the crustal magnetic field over the spectrum of the main field. The crust thus acts as a "magnetic curtain" (Roberts and King, 2013) that hides the higher SH components of the main field at the surface. Consequently, geomagnetic field models may only recover the core field up to degree 13.

These models are built by solving the complex inverse problem of finding the Gauss coefficients that best fit the available data (see e.g. Bloxham et al., 1989; Parker, 1994). The most widely used models are the International Geomagnetic Reference Field (IGRF) models, which up-to-date version is IGRF-12 (Thébault et al., 2015). This model was adopted in December 2014 by Association of Geomagnetism and Aeronomy (IAGA) and represents a weighted average of candidate models computed by several geomagnetic groups in the world (see Thébault et al. (2015) for an exhaustive list). In addition to updating the last version (IGRF-11, Finlay et al. (2010)), it models the main magnetic field of 2015 and offers a prediction for epoch 2015-2020. The prediction of the IGRF-12 model for year 2017 is displayed in figures 1.12 and 1.13, in terms of magnetic field (declination, inclination and 


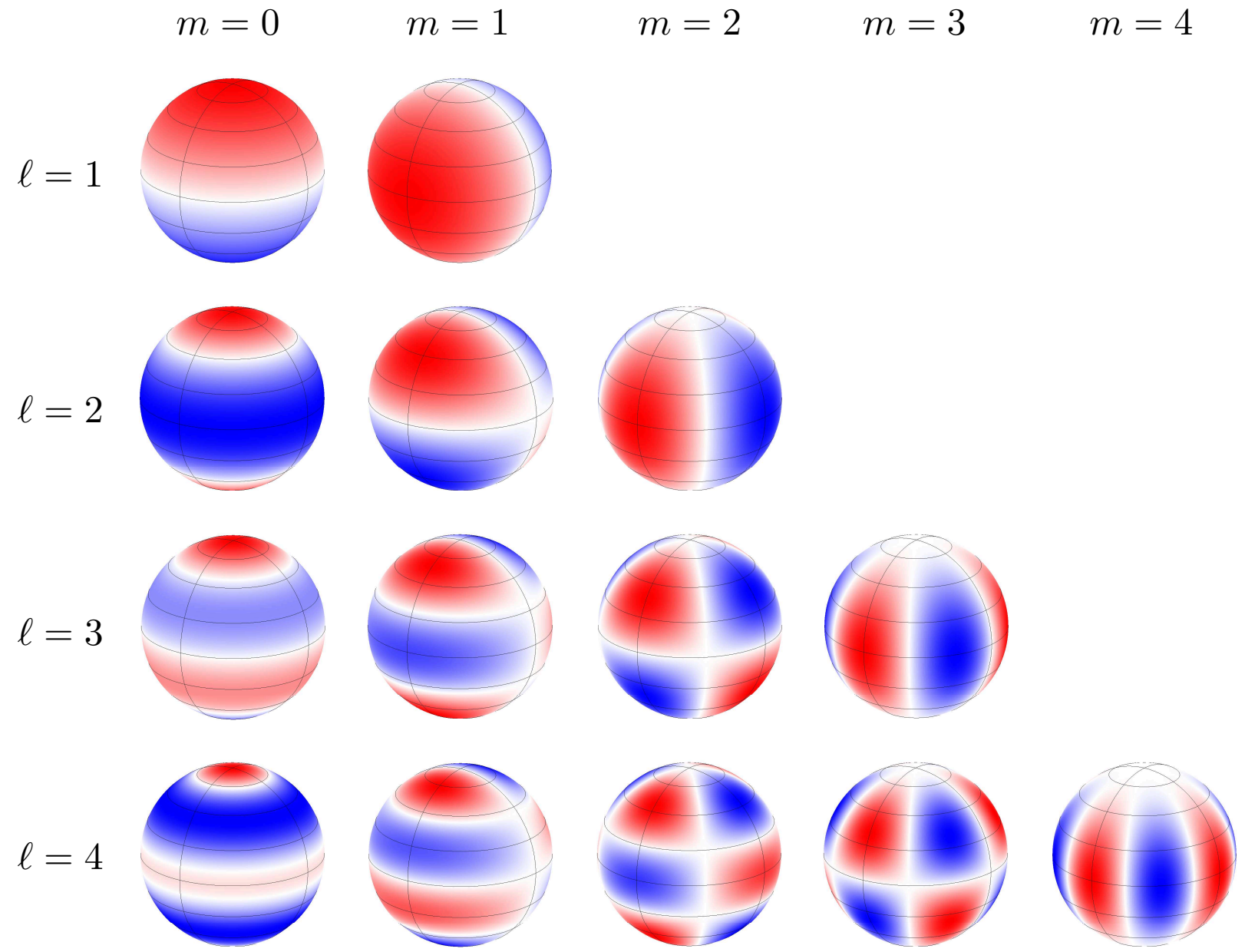

Figure 1.11: Geometrical representation of the spherical harmonic expansion (real part) from degree $\ell=1$ to $\ell=4$. The axial dipole is visible on the top left side $(\ell=1, m=0)$ while the axial dipole is on the right $(\ell=1, m=1)$. Harmonics with $m=0$ are called zonal, sectorial for $\ell=m$ and tesseral in every other cases. 
intensity, from top to bottom) and its annual rate of change, respectively.

The secular variation of the geomagnetic field is however best seen in time-dependent models of the magnetic field at core-mantle boundary. The first models of Cain et al. (1965) and Cain et al. (1967), GSFC(4/64) and GSFC(12/62), used a Taylor series expansion to describe the time variation of the Gauss coefficients, but this method is only adequate for relatively short time spans (see also Langel et al., 1982). The next generation of models thus used Legendre (Bloxham, 1987) or Chebyshev (Bloxham et al., 1989) polynomials and introduced regularization processes, while most recent models use B-spline functions (Bloxham and Jackson, 1992; Jackson et al., 2000) or a combination (Olsen et al., 2006). These models often propose an inversion of the core-flow close to the CMB (see e.g. Bloxham and Jackson, 1991), in which case it is necessary to rely on on two major assumption. The first is the frozen flux hypothesis, also referred to as Alfvén's theorem, and assumed that as the electrical conductivity of iron is high, the magnetic field field lines move as frozen into it. The second hypothesis relies on the fact that the Coriolis force should be dominant in the force balance of the Earth's core. Equating the Coriolis to pressure forces leads to an invariance of the flows along the rotation axis, commonly termed geostrophic. Please note that though a prior information must be introduced to recover a core flow model, due to the non-uniqueness of the solution (Backus, 1968), one may also rely on the "steady-flow" or even "toroidal flow" assumptions. With the advent of the satellite era, the secular variation of the Earth's magnetic field is much more constrained (see e.g. the GRIMM Lesur et al. (2008, 2010) and CHAOS Olsen et al. (2006, 2009, 2010) series of models). However, in order to study the geomagnetic field in historical records, we will often rely on the gufm1 model (Jackson et al., 2000). It reassembles the evolution of the Earth's magnetic field at the core-mantle boundary between 1590 and 1990, by combining the data of mariners, geomagnetic observatories and satellites data (see Jackson et al., 2003, for complementary information on the dataset). From a technical standpoint, the gufm1 expands the epochs modeled by ufm1 (1690-1840) and ufm2 (1840-1990) of Bloxham and Jackson (1992) and improves the resolution of the magnetic field.

\subsection{The geomagnetic westward drift}

"In all the other examples, the needle has gradually moved towards the West"

- E. Halley, 1692

The geomagnetic westward drift was first described by Halley (1692) as a westward variation of the Earth's magnetic field over time. This observation, including worldwide measurements since the beginning of the 17th century, is still the most apparent feature of 
Magnetic Declination for year 2017

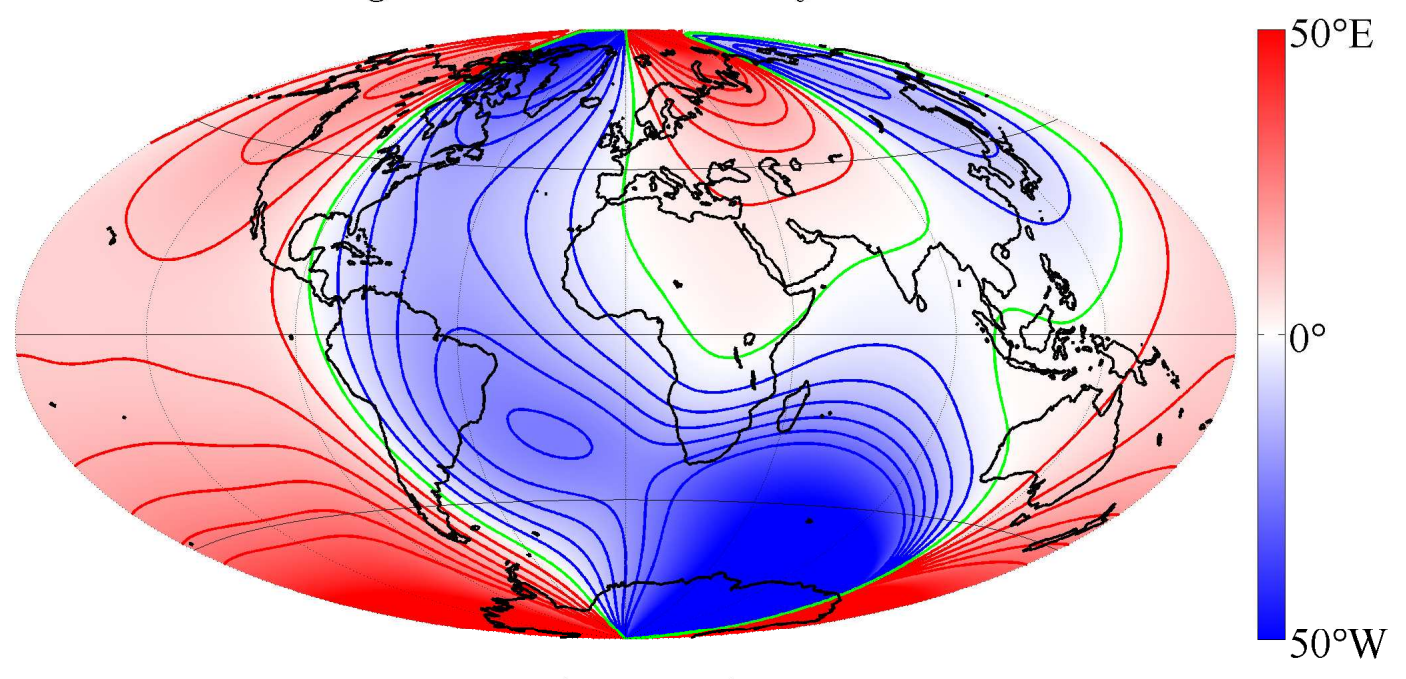

Magnetic Inclination for year 2017

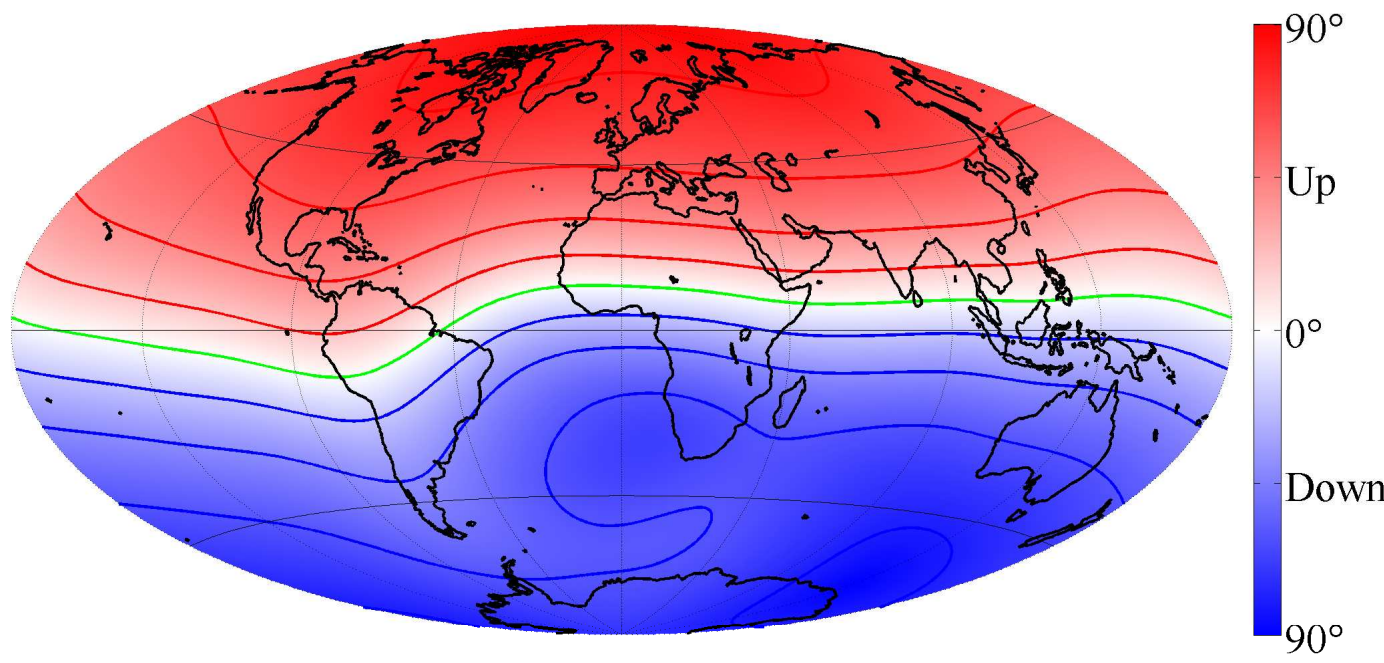

Magnetic Intensity for year 2017

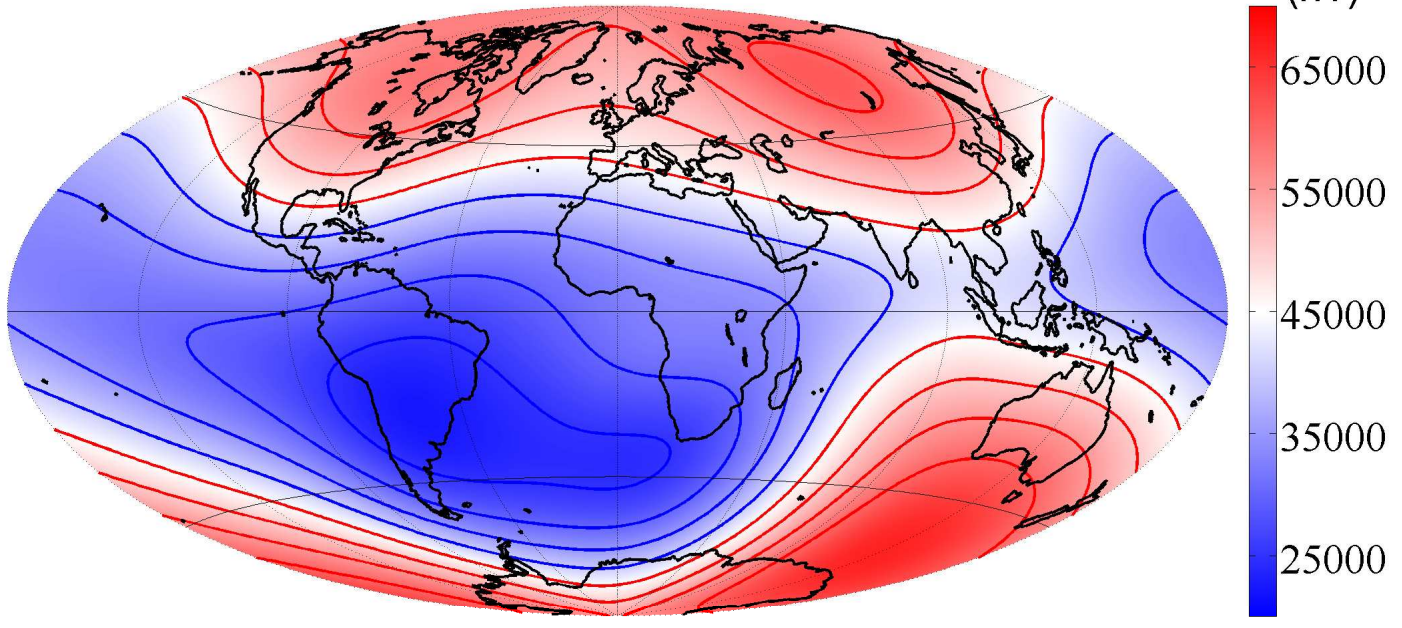

Figure 1.12: Maps of magnetic field of year 2017 of the IGRF-12 model (Thébault et al., 2015). On top, the magnetic declination $D$ with an isocontour spacing of $5^{\circ}$. On the middle, the magnetic inclination $I$ with an isocontour spacing of $20^{\circ}$. The greed line is the zero declination/inclination line. At the bottom, a map of the magnetic intensity $F$ in nanotesla (nT). 
Annual rate of change of the magnetic Declination for year 2017

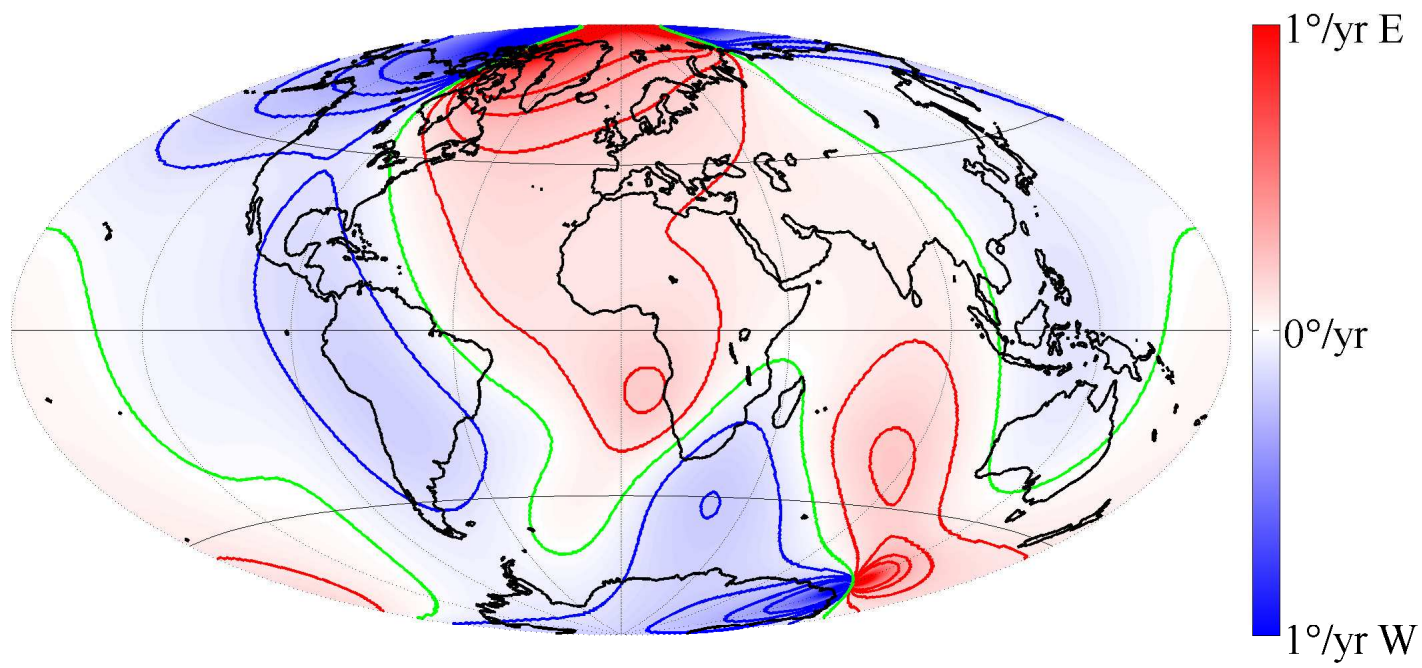

Annual rate of change of the magnetic Inclination for year 2017

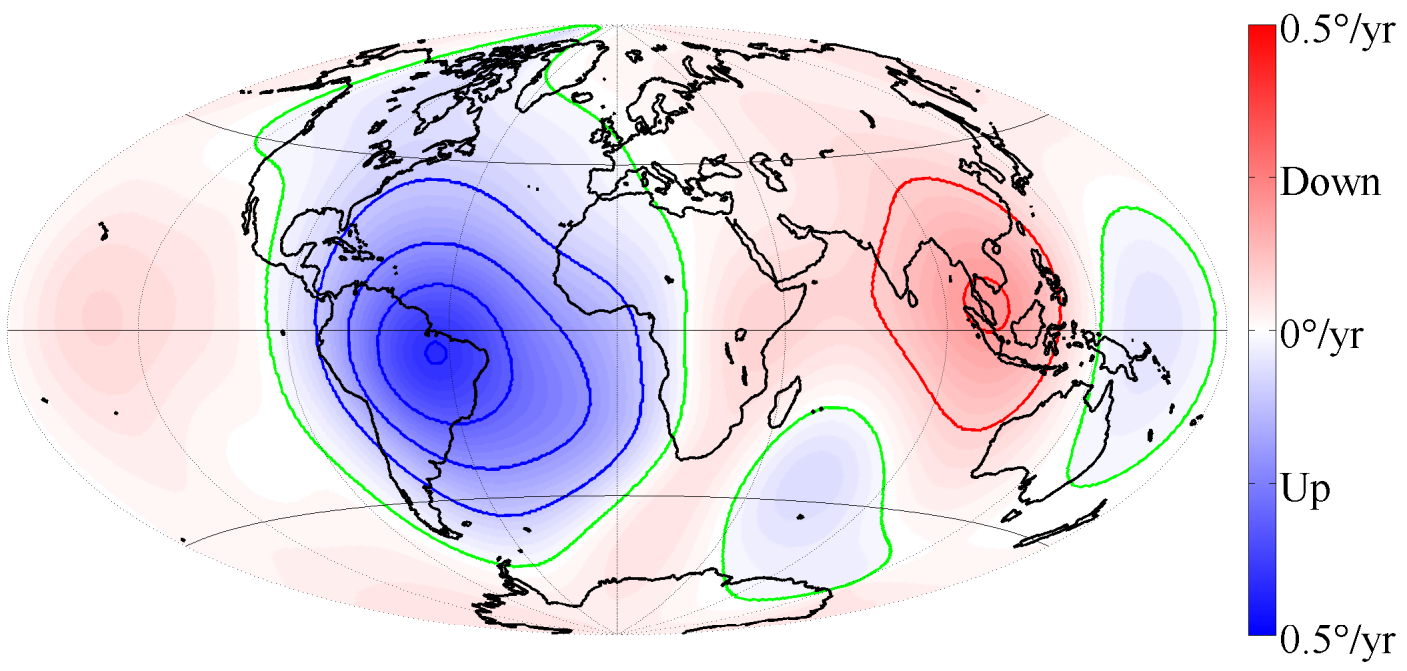

Annual rate of change of the magnetic Intensity for year 2017 (nT/yr)

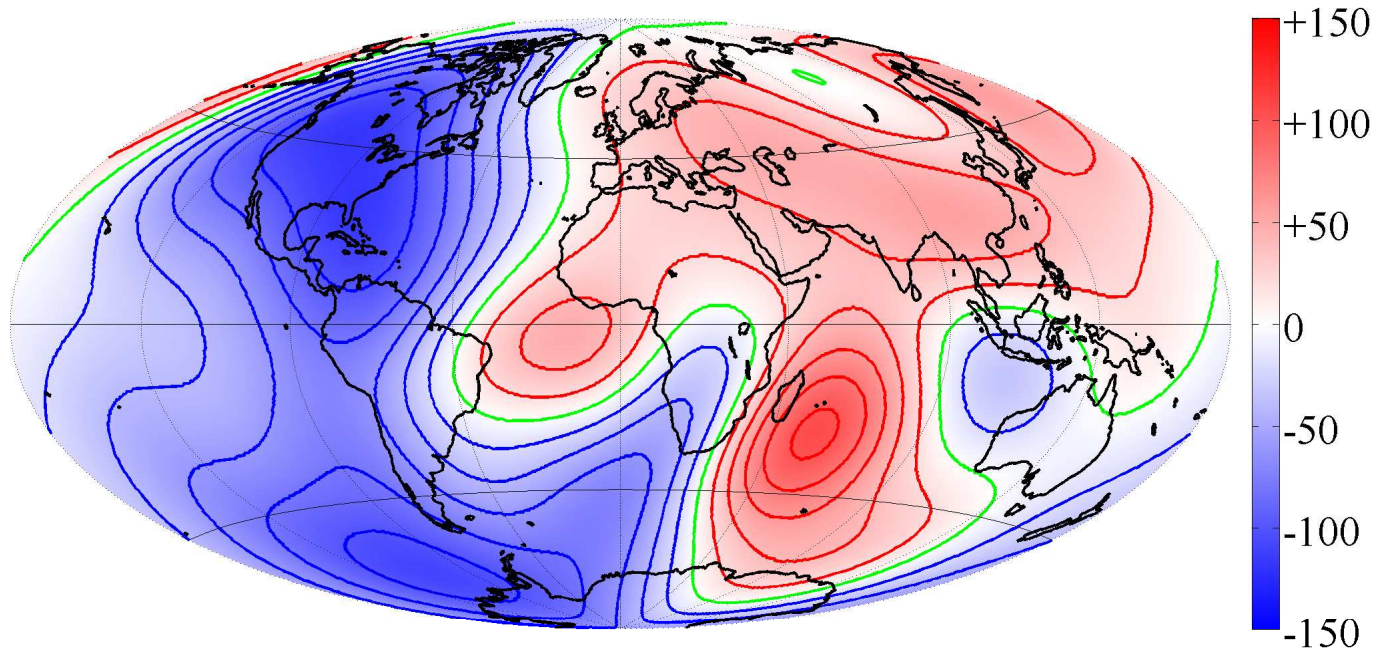

Figure 1.13: Maps of the annual rate of change of magnetic field of year 2017 of the IGRF-12 model (Thébault et al., 2015). On top, the magnetic declination $D$ with an isocontour spacing of $0.1^{\circ} / \mathrm{yr}$. On the middle, the magnetic inclination $I$ with an isocontour spacing of $0.1^{\circ} / \mathrm{yr}$. At the bottom, a map of the annual rate of change of the magnetic intensity $F$ in nanotesla (nT/yr). 
the Earth's magnetic field secular variation. Later on, Bauer (1895) provided one of the first measures of this variation by studying the drift of the agonic lines (zero declination lines of the magnetic field), and found a mean westward drift rate of $0.22^{\circ} \mathrm{yr}^{-1}$ in London. The geomagnetic westward drift was again quantified later with the work of Bullard et al. (1950) and Vestine and Kahle (1968), and estimated as $\sim 0.2^{\circ} \mathrm{yr}^{-1}$, respectively between 1907-1945 and 1910-1965. Both authors distinguished the westward drift of the non dipole field $\left(\sim 0.2^{\circ} \mathrm{yr}^{-1}\right)$ and that of main field $\left(\sim 0.3^{\circ} \mathrm{yr}^{-1}\right)$, and inferred that this drift is the direct result of fluid motion close the core-mantle boundary. More precisely, Bullard et al. (1950) assumed that the outer part of the fluid core (about $200 \mathrm{~km}$ beneath the CMB) should rotate less rapidly than the inner part, resulting in a westward motion relative to the mantle. These works, confirmed by Nagata (1962) and Yukutake (1962), settled the westward drift as a fluctuating but continuous feature of the secular variation of the Earth's field, with an average of $0.2^{\circ} \mathrm{yr}^{-1}$ for the last centuries.
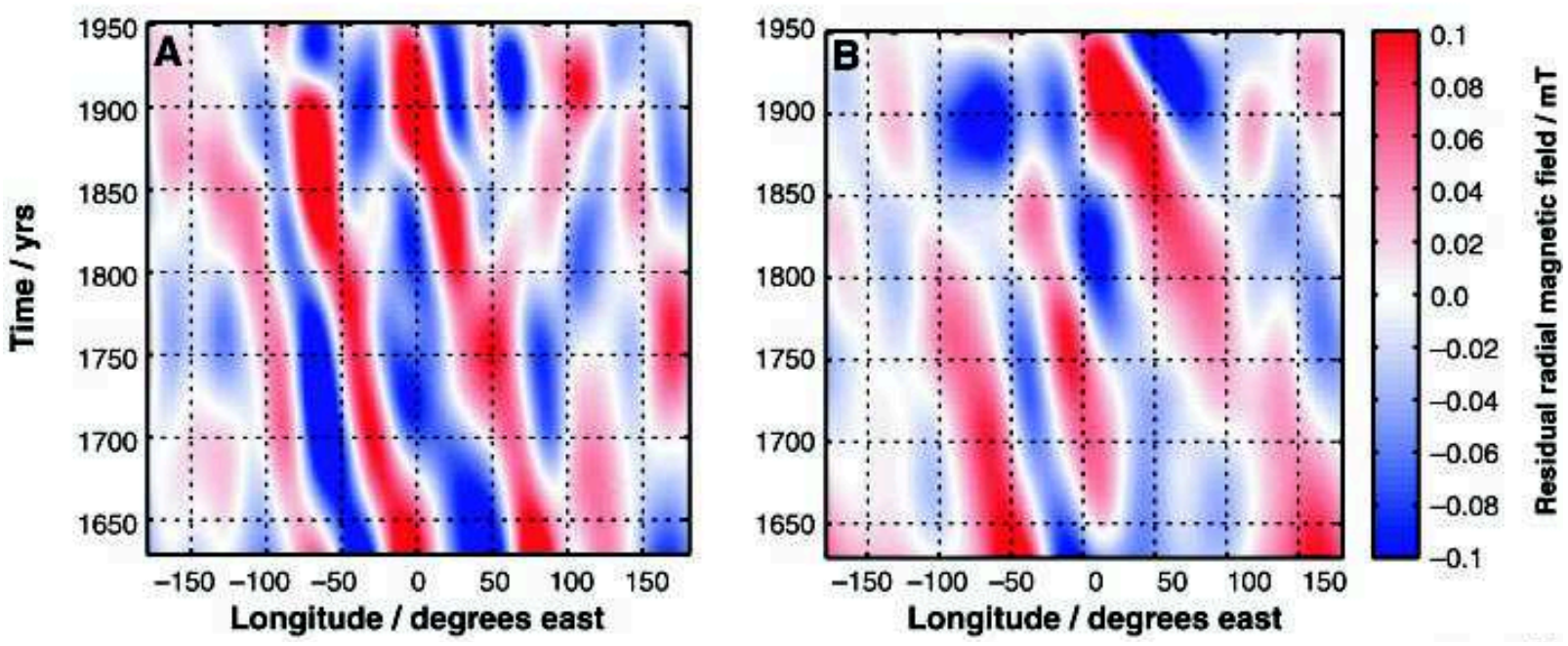

Figure 1.14: Time-longitude diagrams of the non axisymmetric part of the magnetic field at the equator (left) and at $40^{\circ}$ South of latitude, from Finlay and Jackson (2003).

The common point of the earliest models cited in section 1.3.3 is that the secular variation, and thus the geomagnetic westward drift, of the field is more intense in the Atlantic hemisphere, and especially close to the equator. This dependence of the drift was further determined by Jault et al. (1988), who inverted the azimuthal core flow below the CMB from the magnetic data. The interpretation is given within the framework of the torsional oscillations theory developed by Braginsky (1970): the motion of rigid cylinders aligned with the rotation axis. These are actually Alfvèn waves, as the shear produced by a differential rotation between two cylinders results in a restoring Lorentz torque.

The best description of the geomagnetic westward drift over historical records is probably that of Finlay and Jackson (2003). This descriptions is based on the non axisymmetric part of the radial field that vary on timescale smaller than 400 years, referred to as the residual field, from the gufm1 model of Jackson et al. (2000). The evolution of the residual 
field, representing $42 \%$ of temporal variations of the total field at the CMB, is shown in time-longitude diagrams. It expresses the zonal motion of the residual field as a function of time, more vigorous at the equator than at higher latitudes (see figure 1.14). This figure also localizes the drift in the Atlantic hemisphere, the $0^{\circ}$ longitude line being the Greenwich meridian. The latitude dependence of the secular variation is however best seen in figure 1.15, that clearly identifies a peak in the power of the zonal features traveling westward. This peak points the westward drift of the residual field as strongly concentrated around the equator, with a velocity of $17 \mathrm{~km}^{-1}$ over the last 400 years.

Since then, the geomagnetic westward drift is often described as the westward drift of magnetic flux patches, concentrated in the equator of the Atlantic hemisphere, at an average velocity of $0.27^{\circ} \mathrm{yr}^{-1}$ during the last four centuries.

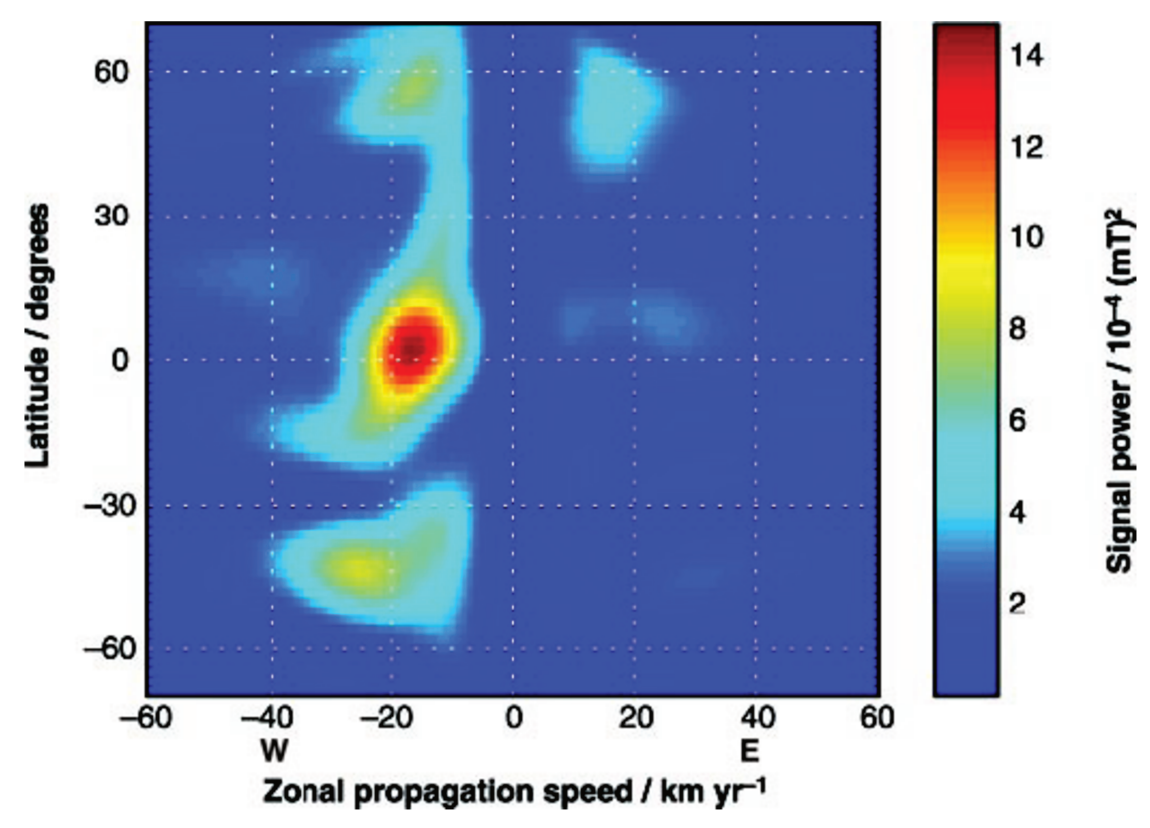

Figure 1.15: Power of the residual field in zonal motion (positive eastward) as a function of the latitude, from Finlay and Jackson (2003).

\subsection{Inner core super-rotation}

\subsubsection{Dynamics}

\section{Driving force}

The inner core super-rotation commonly refers to the possible eastward rotation of the inner core, relative to the mantle. One of the first studies relative to the subject is that of Gubbins (1981). He first inferred that the existence of a strong toroidal magnetic field close to the inner core boundary could produce an electromagnetic torque that tends to rotate the inner core. Indeed, the presence of a strong toroidal field is a natural consequence of differential rotation in a electrically conducting fluid, a mechanism that is nowadays 
recognized as fundamental for the geodynamo action. With a toroidal magnetic field of $10 \mathrm{mT}$ and a dipole field of $0.5 \mathrm{mT}$ close to the inner core boundary, Gubbins (1981) obtained an electromagnetic torque of $10^{19}$ N.m acting on the inner core, that is balanced by an electromagnetic torque of large-scale field on average. The steady state response of the inner core is and eastward rotation of $0.2^{\circ} \mathrm{yr}^{-1}$, which may be accompanied by decadal oscillations.

Since then, the preferred mechanism to entrain the inner core into eastward differential rotation is an electromagnetic torque at the ICB, which requires a strong radial field and eastward motion of the fluid close to the ICB to be effective. Surprisingly, this was observed in the pioneering 3-D numerical simulation of the geodynamo of Glatzmaier and Roberts (1995) and described in a following study (Glatzmaier and Roberts, 1996). Indeed, early geodynamo simulations consistently exhibit an eastward rotation of the inner core of $2^{\circ}$ to $3^{\circ} \mathrm{yr}^{-1}$, associated with a specific flow pattern commonly named "polar vortices" (see figure 1.20). In the tangent cylinder ${ }^{3}$, the buoyancy forces generated by the release of light elements produce a large-scale flow that is prograde (eastward) and toward the rotation axis close to the ICB, and becomes progressively retrograde (wesward) and outward the rotation axis when approaching the CMB. These large scale flows are responsible for the shear of the poloidal magnetic field lines that produce a strong toroidal field inside the tangent cylinder. They are nonetheless well represented by the balance of the Coriolis, Buoyancy and pressures forces : the thermal wind balance. The presence of this large-scale flow is supported by experiments on rotating fluids (Aurnou et al., 2003) and is consistent with the presence of anti-cyclonic flow in polar regions inverted from geomagnetic observations (Olson and Aurnou, 1999; Hulot et al., 2002), though the lack of data may leads to poorly resolved areas (Eymin and Hulot, 2005). It is also supported by numerical simulations of the geodynamo, which often exhibit this flow pattern spontaneously (Aubert, 2005; Sreenivasan and Jones, 2006).

Hence, numerical simulations featuring this flow and considering the inner core as electrically conducting do yield a prograde rotation of the inner core. This differential rotation operates at a weighted average of the angular velocity of the fluid close to the ICB, as firstly observed by Glatzmaier and Roberts (1996). Indeed, the electromagnetic torque at the ICB the result of different contributions. This mechanism was investigated by Aurnou et al. (1996) in simplified models of the flows in the tangent cylinder. The azimuthal velocity profile extracted from the thermal wind balance is shown to produce a toroidal magnetic field from the shear of the imposed poloidal field that is added to the toroidal field produced by the velocity jump at the inner core boundary. As a consequence, the inner core, of radius $r_{\mathrm{i}}$, is found to lag the fluid at the ICB of $r_{\mathrm{i}} / 4 D \approx 14 \%$ of angular velocity, with $\mathrm{D}$ the thickness of the fluid outer core. In addition, as the azimuthal velocity of a thermal wind is mostly governed by the temperature gradient (also valid for chemical convection), it is possible to retrieve the average inner core differential rotation by guessing

3. an imaginary cylinder aligned with the rotation axis and tangent to the inner core 
the latter parameter. For relatively small temperature gradient across the tangent cylinder $\left(5 \times 10^{-4} \mathrm{~K}\right.$ and $\left.1.3 \times 10^{-3} \mathrm{~K}\right)$, and in the context of his simplified model, Aurnou et al. (1996) found inner core rotation of $1.1^{\circ} \mathrm{yr}^{-1}$ and $3^{\circ} \mathrm{yr}^{-1}$ corresponding to toroidal fields of $24 \mathrm{mT}$ and $66 \mathrm{mT}$ close to the ICB. This concept was further improved in a subsequent study (Aurnou et al., 1998) considering three core flow models, inside and outside the tangent cylinder. The thermal wind flow inside the tangent cylinder are consistently found to generate an eastward inner core rotation on the order of $1^{\circ} \mathrm{yr}^{-1}$, while a global westward motion of the fluid outer core outside the tangent cylinder only trigger a retrograde rotation $0.013^{\circ} \mathrm{yr}^{-1}$.

Another driving mechanism, though still based on the Lorentz force, is to relate the time-dependent inner core differential rotation to the presence of torsional oscillations in the core (Braginsky, 1970). Indeed, the oscillations of rigid cylinders in the core should exert a Lorentz force on the tangent cylinder, and thus on the inner core (Mound and Buffett, 2003). This was investigated in geomagnetic observations by Zatman (2003), who inferred that the inner core had endured an eastward rotation of $0.1^{\circ} \mathrm{yr}^{-1}$ between 1970 and 1990 .

\section{Braking}

The suggestions that the inner core is rotating eastward at an angular velocity greater than $1^{\circ} \mathrm{yr}^{-1}$ were questioned by Buffett (1996b) and the introduction of a presumed gravitational coupling between the inner core and the mantle, that was not considered in early numerical simulations. This gravitational coupling is the result of non-axially symmetric density anomalies in the mantle, that deform the surface of constant potential inside the core. The main feature of these anomalies is the flattening of the Earth at the equator is, for example, responsible for a deviation of the isopotential lines of gravity of a hundred meters close to the ICB (Forte and Peltier, 1991). Such anomalies are expected to influence the density structure of the inner core, which in return is necessarily locked to the mantle. Then, if an electromagnetic torque acts on the inner core, the gravitational coupling is a restoring torque that forces the inner core to remain aligned with the mantle (see figure 1.16).

The only way to conciliate both the inner core differential rotation and the gravitational coupling exerted on the latter is to assume that the inner core is able to undergo viscous deformations as it rotates (Buffett, 1997). This scenario is compatible with inner core angular velocities on the order of $1^{\circ} \mathrm{yr}^{-1}$ if its bulk viscosity is less than $3 \times 10^{16} \mathrm{~Pa}$.s or if the deformation only concerned a layer of a hundred meters at the top of the inner core, having a viscosity lower than $2 \times 10^{14}$ Pa.s. In that case, the inner core is able to undergo both steady and fluctuating rotation, while deforming to remain align with the mantle. Another envisioned scenario is that the viscosity of the inner core is greater than $1.5 \times 10^{20}$ Pa.s, but the latter includes a complete locking of the inner core in the case where the rotation had ever slow down. Numerical simulations of the geodynamo including such a mechanism thus report much lower rotation rates of inner core, relative to the 

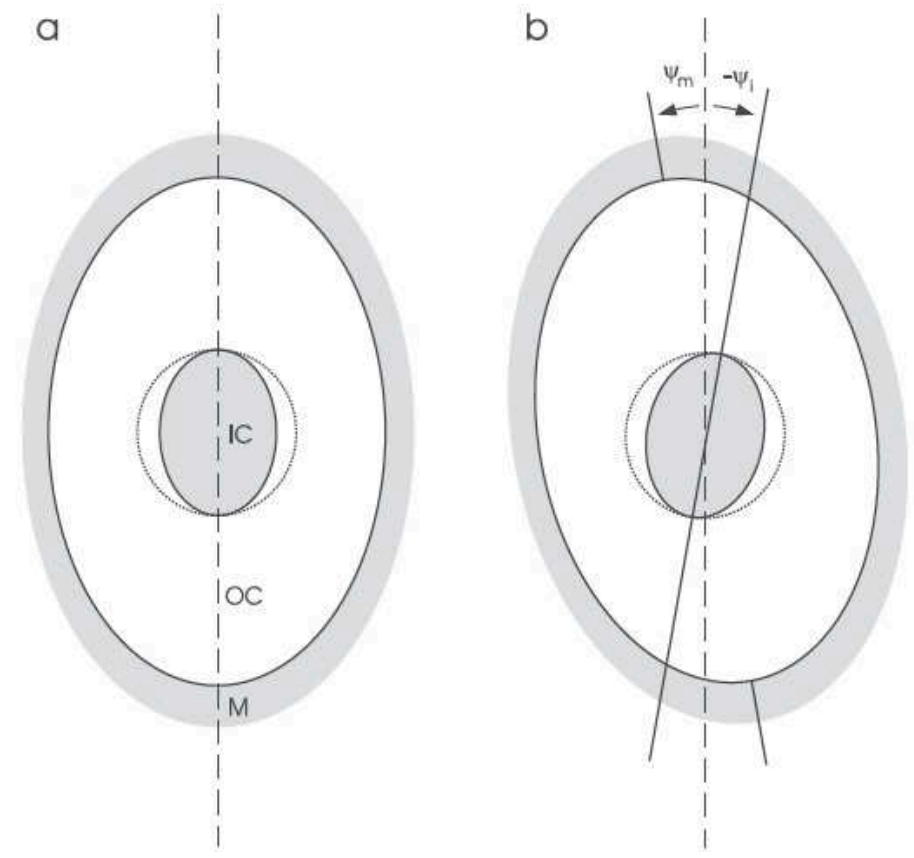

Figure 1.16: Equatorial view of the inner core (IC), outer core (OC), mantle (M) system. (a) The inner core (deformed) is at equilibrium with the mantle (M). (b) The inner core is misaligned with the mantle, resulting in a gravitational torque proportional to the misalignment angle, the strength of density anomalies in the mantle and the inner core viscosity. From (Mound and Buffett, 2003).

mantle. Buffett and Glatzmaier (2000) found a steady rotation rate of $0.02^{\circ} \mathrm{yr}^{-1}$, using hyperdiffusivity to solve the dynamics of the outer core (as Glatzmaier and Roberts (1995)) and free-slip mechanical boundary conditions at the ICB, Aubert and Dumberry (2011) reported a steady rotation of a few degrees per millions years, with a standard treatment of viscosity and no-slip boundary conditions at the ICB. However, both authors do not exclude a time-dependent inner core differential rotation, which may be on the order of $0.1^{\circ} \mathrm{yr}^{-1}$.

\section{Observational constraints}

The main constraint on the differential rotation of the inner core is the observed variations of the length of day (LOD, see e.g. Gross, 2007, for a review). The consideration of a gravitational coupling between the inner core and the mantle was besides invoked in order to explain the decadal variations of the LOD (Buffett, 1996a). Indeed, if the inner core experiences a given gravitational torque, then the mantle necessarily experiences the exact opposite torque. Therefore, the rotation of the inner core is bounded by the observation of LOD variations. As an example, the 6-yrs oscillation period of the LOD was first attributed to mantle-inner core gravitational (MICG) modes that arises from the non hydrostatic shape of the Earth (Mound and Buffett, 2006). However this conclusion, as well as any inference on the rotation of the inner core from dynamical models, is highly sensitive to the chosen values of the inner core viscosity and the intensity of density anomalies in 

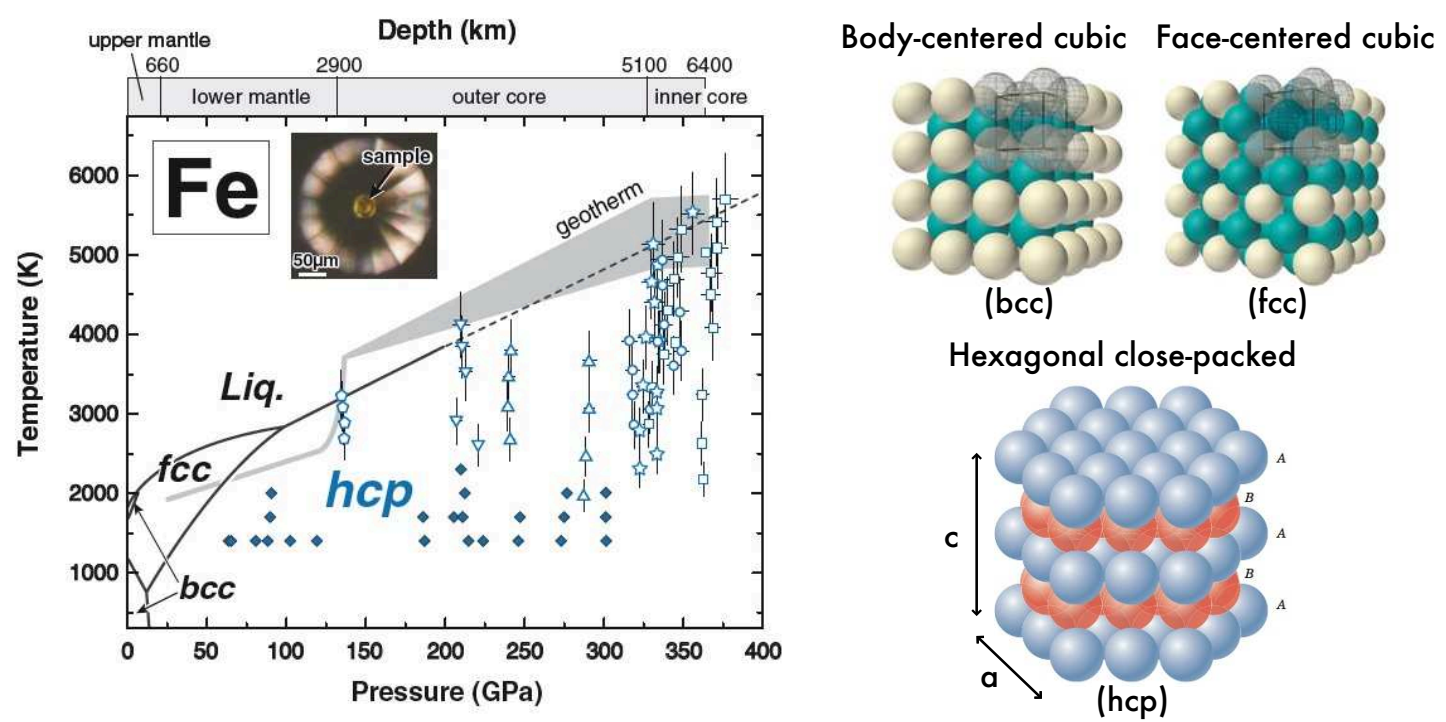

Figure 1.17: Phase diagram of Fe from Tateno et al. (2010) on the left, and structure of the body-centered cubic (bcc), face-centered cubic (fcc) and hexagonal close-packed (hcp) phases, on the right.

the mantle, which are both deep Earth parameters poorly constrained. Also, the LOD variations are sensitive to direct coupling between the core and the mantle such that, at the time, the favored scenario to explain the 6-yrs oscillations on the LOD signal is the presence of fast torsional oscillations (Gillet et al., 2010) (with electromagnetic coupling between the core and the mantle).

\subsubsection{Seismological evidence}

The seismological evidences of a differential rotation of the inner core relative to the mantle are based on two properties of the inner core: its anisotropy and its lateral variations (or tilt in the anisotropy). The anisotropy of the inner core was first measured by Poupinet et al. (1983), who observed that PKIKP ${ }^{4}$ waves propagating North-South arrived two seconds before PKIKP waves propagating in the equatorial plane, though the interpretations involved faster regions around the inner core poles or an inner core eccentricity. The link between the inner core anisotropy and the difference in travel time between equatorial and polar path of $\mathrm{P}$ waves was demonstrated by Morelli et al. (1986) with body waves, and Woodhouse et al. (1986) with normal modes. The magnitude of this anisotropy was later confirmed to be around $3 \%$ by Creager (1992) and Tromp (1993).

The anisotropy of the inner core is a direct consequence of pressure/temperature dependence of iron mineralogy, presented in figure 1.17. At standard temperature and pressure (STP), the stable phase of iron is the body-centered cubic phase (bcc), an arrangement that changes to a stable faced-centered cubic phase (fcc) as the temperature

4. $\mathrm{P}$ waves that travel across the mantle $(\mathrm{P})$, the outer core $(\mathrm{K})$, the inner core $(\mathrm{I})$ and continuing across the outer core $(\mathrm{K})$ and the mantle $(\mathrm{P})$. 
increases. At high pressure, the stable phase is a hexagonal close-packed form (hcp) which interesting property is the ratio (c/a) between the cylindrical axis of symmetry (c-axis) and the axis perpendicular to it (a-axis). The first ab initio computations favored the hcp phase of pure iron at the conditions of the core (Vočadlo et al., 2000), though the other phases (notably the bcc phase) may be stable considering the presence of lighter elements impurities , such are Sulphur of Silicon (Vočadlo et al., 2003; Belonoshko et al., 2003). This was later confirmed with diamond anvil cell experiments that were able to reach the extreme conditions of the core, and establish the stability of the hcp phase for pure iron, iron-nickel alloy and iron with silicon impurities (Tateno et al., 2010, 2012; Sakai et al., 2011). These high-pressure high-temperature also confirmed that, at the inner core conditions, the ratio $c / a$ of hcp iron is smaller (c/a=1.602 for Tateno et al. (2010)) than the ideal value of $\mathrm{c} / \mathrm{a}=1.6229$, as inferred by Stixrude and Cohen (1995). This ensures that the c-axis of hcp iron at core conditions is the "fast" axis for wave propagation, so that an inner core composed of hcp iron aligned with the rotation axis perfectly explain the discrepancy between the arrival times of the equatorial and polar paths of PKIKP waves observed by Poupinet et al. (1983), though the mechanism of preferred alignment is still debated.

The early reports of the inner core super-rotation of Song and Richards (1996) were based on differential travel time between $\mathrm{PKP}(\mathrm{BC})^{5}$ and PKIKP waves, which is explained by the inner core anisotropy and its tilt from the rotation axis inferred by other seismological studies (Shearer and Toy, 1991; Creager, 1992; Su and Dziewonski, 1995). This differential travel time is found to change over time and is interpreted as a rotation of the inner core relative to the mantle of $1.1^{\circ} \mathrm{yr}^{-1}$ by Song and Richards (1996) and $3^{\circ} \mathrm{yr}^{-1}$ by Su et al. (1996) for the second half of the century. This view was later questioned by the observations of hemispherical variations in the structure of the inner core (Tanaka and Hamaguchi, 1997), that better explained body waves propagations data than a tilt in the inner core anisotropy (Irving and Deuss, 2011), and normal studies reporting westward (Sharrock and Woodhouse, 1998) or smaller eastward rotation of $0 \pm 0.2^{\circ} \mathrm{yr}^{-1}$ (Laske and Masters, 1999) and $1.13 \pm 0.11^{\circ} \mathrm{yr}^{-1}$ (Laske and Masters, 2003).

Measurements of differential travel times of PKIKP and PKP $(\mathrm{BC})$ waves are also subject to strong mantle and D" heterogeneities, noise, and source localization, that challenge the early reports of the inner core super-rotation. Thus, improvements were made by the next generation of seismological studies, concerning the quality of data and the earthquake selections. These studies generally interpret the difference in travel time between earthquake doublets ${ }^{6}$ or pair-events as the differential rotation of hemispherical anomalies of the inner core. Zhang et al. (2005) thus reported eastward rotations rates between $0.3^{\circ} \mathrm{yr}^{-1}$ and $0.5^{\circ} \mathrm{yr}^{-1}$, in agreement with Song and Poupinet (2007), while others studies rather suggest a zero average rotation (Souriau and Poupinet, 2000) or at least smaller than $0.2^{\circ} \mathrm{yr}^{-1}$ (Poupinet et al., 2000). In addition, Mäkinen and Deuss (2011) computed two different

5. $\mathrm{P}$ waves that travel across the outer core, close to the inner core.

6. Two Earthquakes that happened at two different dates, at the same place and with the same focal mechanism. 


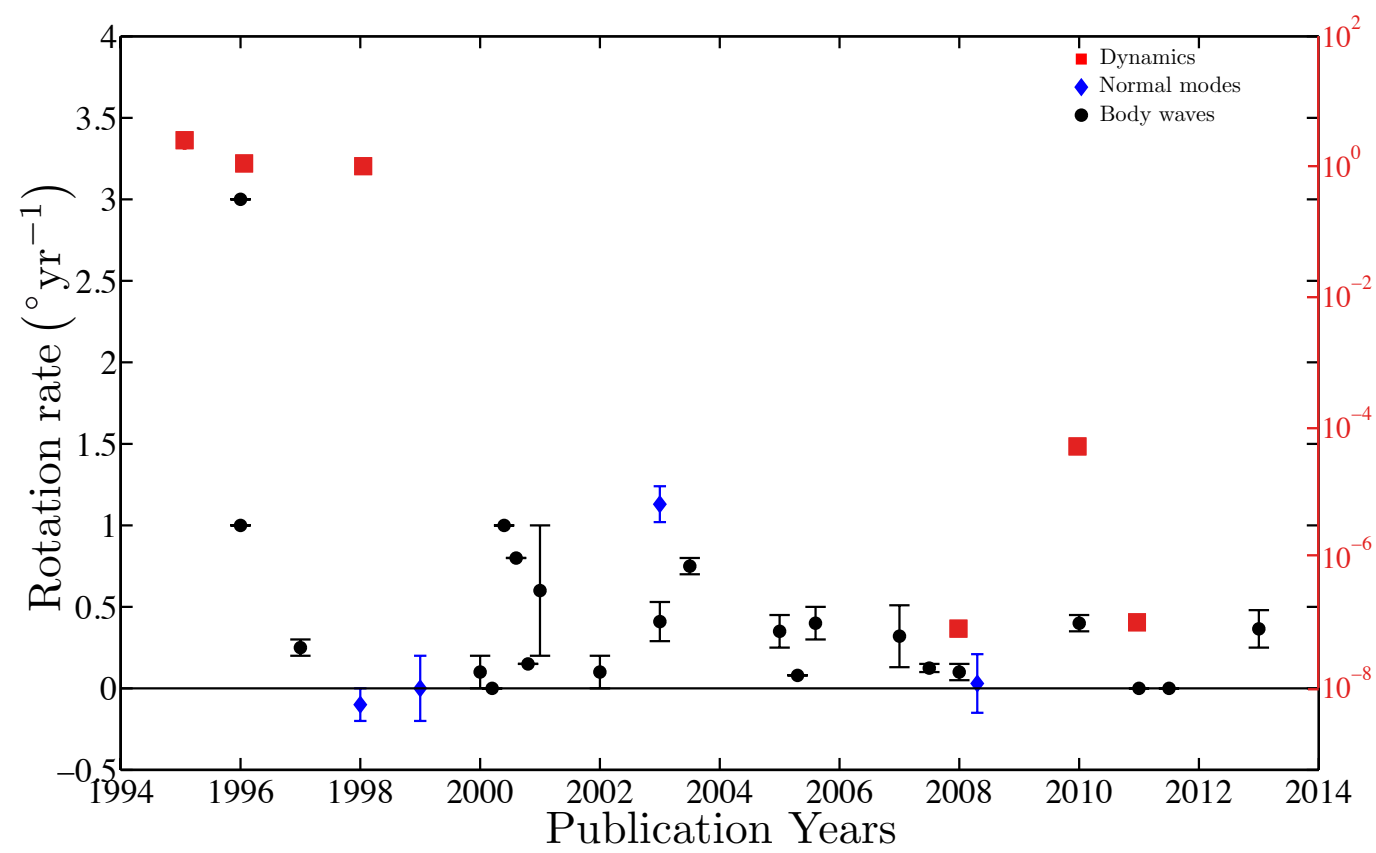

Figure 1.18: Inferred inner core rotation rates relative to the mantle from Dynamical studies (red squares), normal modes studies (blue diamonds) and body waves studies (black rounds), inspired by Deuss (2014).

rotations rates from two closely spaced stations in Alaska and thus precluded any differential rotation of the inner core, relative to the mantle.

Although we can observe a global consensus throughout the years that tends to diminish the early estimates of the inner core super-rotation of Song and Richards (1996) and Su and Dziewonski (1995) to a few tenth of degree per years, the seismological evidences are various (see figure 1.18). One way to reconcile theses different observations is to consider that they reflect the fluctuations of the differential rotation of the inner core over time. This was explored by Tkalčić et al. (2013) who reported and average super-rotation of the inner core between $0.25^{\circ} \mathrm{yr}^{-1}$ and $0.48^{\circ} \mathrm{yr}^{-1}$ from 1961 to 2007 , accompanied by decadal oscillations of about $1^{\circ} \mathrm{yr}^{-1}$.

\subsection{Objectives of this work}

\subsubsection{Coupled-Earth dynamo}

Substantial progress in geodynamo simulations were brought by Aubert et al. (2013) and the development of the Coupled-Earth (CE) Dynamo model. This model is able to generate an Earth-like magnetic field, in the sense of Christensen et al. (2010), and reproduces the main feature of its secular variation as observed in historical records: the geomagnetic westward drift. The inner core is considered as electrically conducting and the mechanical boundary condition at the ICB is no-slip while the mantle is considered as insulating, with free-slip boundary condition at the CMB. Both mantle and inner core are free to axially 
rotate, though there is no direct coupling between the mantle and the outer core. The only torque exerted on the mantle arises due to gravitational coupling with the inner core, which submitted to the influence of electromagnetic, viscous and gravitational torques. As the mantle only experiences a gravitational torque, the latter necessarily vanish in the long-term, in order to ensure the conservation of angular momentum. In such a configuration, the inner core is forced to remain aligned with the mantle on time average, and the resulting large-scale outer core flow consists in a westward gyre close to the CMB (see also Aubert, 2013). This quasi-geostrophic gyre, the existence of which was inferred from core flow inversions (Pais and Jault, 2008; Gillet et al., 2009), generates a shear that is sufficient to concentrate the azimuthal magnetic field lines close to the $\mathrm{CMB}$, in equatorial regions. This mechanism thus produces a steady geomagnetic westward drift of $14 \mathrm{~km} \mathrm{yr}^{-1}$, close to the observed westward drift in historical records $\left(17 \mathrm{~km} \mathrm{yr}^{-1}\right.$, Finlay and Jackson, 2003), with the appropriate latitudinal dependence (see figure 1.19).

Finally, the localization of the drift in the Atlantic hemisphere is achieved by implementing a heterogenous inner core growth. A heterogenous inner core growth was first modeled by Aubert et al. (2008) in order to explain the hemispherical seismic anomalies at the top of the inner core (Tanaka and Hamaguchi, 1997; Niu and Wen, 2001), as the freezing rate of iron is supposed to influence its degree of anisotropy through texturing during solidification (Bergman et al., 2003, 2005). Regions with lower freezing rates should then correspond to more anisotropic regions. In Aubert et al. (2008) the differential growth of the inner core is induced by lateral variations in the CMB heat flux inferred by seismological studies (McNamara and Zhong, 2005; der Hilst et al., 2007). This top-down forcing promotes faster freezing rate in the eastern hemisphere of the inner core, resulting in a large-scale thermal wind circulation in the core through the release of light elements during freezing. In Aubert et al. (2013), the heterogenous inner core growth is directly implemented as a boundary condition at the ICB and dominates the thermal mantle-induced effects. As a consequence, the equatorial gyre is distorted into an asymmetric path that is closer to the ICB where the release of light element is more intense. This region of the inner core corresponds to that below Indonesia, which necessarily bring the gyre to reach the CMB below the Atlantic. Though it is not in agreement with an inner core translation, as proposed by Monnereau et al. (2010) to explain the hemispheric anomalies of the inner core's anisotropy, this scenario offers combined evidences to both seismological observations and the localization of the geomagnetic westward drift in the Atlantic hemisphere (see figure 1.19).

\subsubsection{Direct core-mantle coupling}

The work of Aubert et al. (2013) notably underlines the importance of angular momentum conservation arguments for the dynamics of the inner/outer core-mantle system. However, it neglects the potential influence of a direct coupling between the fluid outer core and the mantle. This coupling may be of different nature, which is besides a long standing debate, but a constraint on its magnitude can be obtained through the observation of the 

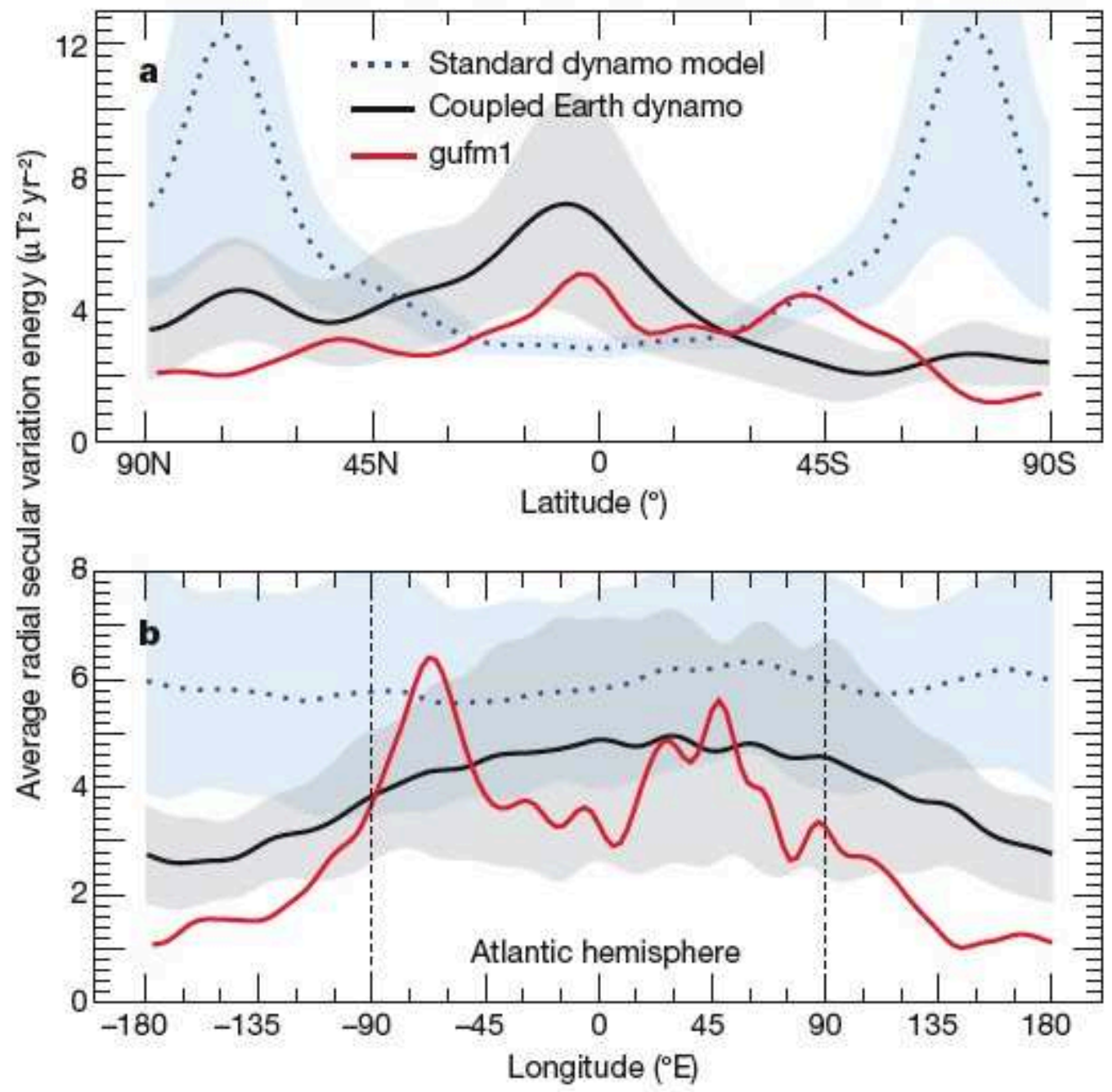

Figure 1.19: Profiles of 400-years time averages radial secular variation energy, as a function of latitude (top) and longitude (bottom), for a standard dynamo model (blue dots), the CE dynamo model (black line) and the gufm1 model (red line) (Jackson et al., 2000). Outputs of numerical simulations are filtered at spherical harmonic degree and order 8 , and shaded areas correspond to the standard deviation. From Aubert et al. (2013). 
LOD variations (see Gross, 2001, 2007). The general agreement thus stipulates that the candidate torque acting on the mantle to explain the LOD variation must be of the order of $10^{18} \mathrm{~N} \mathrm{~m}$.

\section{Viscous Torque}

A viscous torque may arise from the friction between the fluid outer and the mantle, in which case the latter should be dragged in the direction of the subsurface flows. However, the viscous torque is directly proportional to the fluid's kinematic viscosity, which is around $10^{-6} \mathrm{~m}^{2} \mathrm{~s}$ for the outer core (Poirier, 1988; de Wijs et al., 1998; Terasaki et al., 2006). Assuming typical core flow velocities of a few millimeters per seconds then lead to a viscous torque on the order of $10^{15} \mathrm{~N} \mathrm{~m}$, which three orders of magnitude than the required torque. In numerical simulations, as the calculations of small-scale turbulence is numerically demanding, such low viscosity values are not attainable yet. Thus, the viscosity of the fluid is over-estimated by several orders magnitude, an so is the viscous torque. To render the fact that the viscous torque should, in theory, be not sufficient to explain the LOD variations, we will impose stress-free boundary conditions at both ICB and CMB.

\section{Topographic Torque}

It was first suggested by Hide (1969) that the LOD variations may be explained by topographic torque acting on the mantle, arising from pressure forces of the fluid outer core flows on non axisymmetric bumps of the CMB. This torque, and its estimate, is thus proportional to core flows close to the CMB and the CMB topography (see e.g. Roberts, 1988; Buffett, 1998; Jault and Mouël, 1989). In the framework of torsional oscillations in the core inferred from geomagnetic field models of (Jault et al., 1988) and considering CMB bumps on the order of five kilometers suggested by Morelli and Dziewonski (1987), Jault and Le Mouël (1991) found a topographic torque on the order of $10^{19} \mathrm{~N} \mathrm{~m}$. On the other hand, dynamical estimates of this torque in 3-D models of the geodynamo of Kuang and Chao (2001) showed that the amplitude of the CMB topography should be greater than three kilometers for the torque to be effective, which contrast with recent estimates of peak-to-peak amplitudes of one kilometers by Tanaka (2010). We thus follow the conclusion of Roberts and Aurnou (2012) that there is no clear evidence that the topographic torque may explain the LOD variations so far, though the opposite may also be true, and exclude the possible effects of CMB topography on the conservation of the mantle angular momentum.

\section{Electromagnetic Torque}

In the case where the mantle is not perfectly insulating, electric currents flowing from the core may induce an electromagnetic torque on the mantle. This mechanism was first proposed by Bullard et al. (1950) as a link between the geomagnetic westward drift and 
the variations of the LOD. Later, Rochester (1960) demonstrated that the electromagnetic torque may be of the right order of magnitude if the mantle has an electrical conductivity of $10^{2} \mathrm{~S} \mathrm{~m}^{-1}$ in the $2000 \mathrm{~km}$ above the CMB, corresponding to a conductance of $2.10^{8} \mathrm{~S}$. This value is still considered as a limiting factor to explain the LOD variations by electromagnetic coupling and was confirmed by Stix and Roberts (1984), who studied the fluctuations of this torque over time. The existence an electromagnetic torque at the CMB is only constrained by the presence of an electrically conducting layer at the base of the mantle. The electrical conductivity of the mantle is often inferred by modeling the induced magnetic field in the mantle from external sources, as performed by Civet et al. (2015) with one year of Swarm data. The electrical conductivity is found to increase from $10^{-3} \mathrm{~S} \mathrm{~m}^{-1}$ at $400 \mathrm{~km}$ below the surface, to $\simeq 4 \mathrm{~S} \mathrm{~m}^{-1}$ at $2000 \mathrm{~km}$ depth, which is not sufficient to reach the $10^{8} \mathrm{~S}$ value of mantle conductance. Morever the solution generally looses accuracy as a function of depth, so that the conductivity of the lowermost mantle is still poorly constrained. However, a highly conducting lower mantle is in agreement with several high-pressure/high-temperature mineral experiments. The lower mantle may indeed be composed of a thick (200-300 km) layer of post-perovskite (Murakami et al., 2004) of high conductivity (Ohta et al., 2008), or even a thin layer of metallic FeO having the same electrical conductivity as the Earth's core (Ohta et al., 2012, 2014). The latter assumption is favored by dynamical models, as necessary to explain the out-phase component of the forced nutation of the Earth (Buffett, 1992; Buffett et al., 2002). Further constraints on the electrical conductivity of the lower mantle may finally reside in the observation and the analysis of geomagnetic jerks: abrupt changes in the secular variation (or secular acceleration) of the magnetic field from internal origins (see e.g. Bloxham et al., 2002). The very observation of the geomagnetic jerks indeed place a upper bound on the lower mantle conductances being lower than $2.5 \times 10^{9} \mathrm{~S}$ (Alexandrescu et al., 1999; Dumberry, 2007). Recently, the identification of intradecadal variations in the LOD (Holme and De Viron, 2013) and their link with geomagnetic jerks also suggested a conducting layer of thickness lower than $50 \mathrm{~km}$, thus encouraging the highly conducting/thin layer assumption.

In view of these arguments, we will therefore consider in the following that the direct coupling between the mantle and the fluid outer is of electromagnetic origin, and neglect any other source of coupling at the core-mantle boundary.

\subsubsection{PhD work}

This PhD work is based on the Coupled-Earth dynamo model developed by Aubert et al. (2013) and described in section 1.6.1, in which we add an electromagnetic torque coupling the mantle and the outer core flow close to the core-mantle boundary and remove viscous torques at ICB. Though there are alternative theories to explain the westward drift such as magnetic winds (Livermore et al., 2013) or magnetic Rossby waves (Hori et al., 2015), this self-consistent convective model offers a complete description of the main features of the geomagnetic secular variation. The objective is to obtain better constraints on the inner 
core super-rotation than those inferred by seismological studies. Our approach consists in expressing the link between the well-known geomagnetic westward drift and the inner core super-rotation. This link may be obtained by the conservation of the angular momentum of the mantle/outer core/inner core system, and the formulation of dynamical expressions of the electromagnetic torques. These expressions are validated in numerical simulations of the geodynamo, the set-up of which is summurized in figure 1.20.

The manuscript is organized as follows. Chapter 2 summarizes the model used in this $\mathrm{PhD}$ work, while its numerical implementation is displayed in chapter 3. Chapter 4 is a reproduction of EPSL paper (Pichon et al., 2016) which analyzes the long-term rotational dynamics of the coupled system. The first approach of the fluctuating system is the expression of time-dependent models of the electromagnetic torques at the fluid core boundaries in chapter 5 , which are validated in case-study numerical simulations in chapter 6 . From this analytical and numerical study follows an application to the amplitudes of the time-dependent rotational dynamics of the system in geodynamo simulations in chapter 7 . Eventually, chapter 8 outlines the main conclusions of this work. 


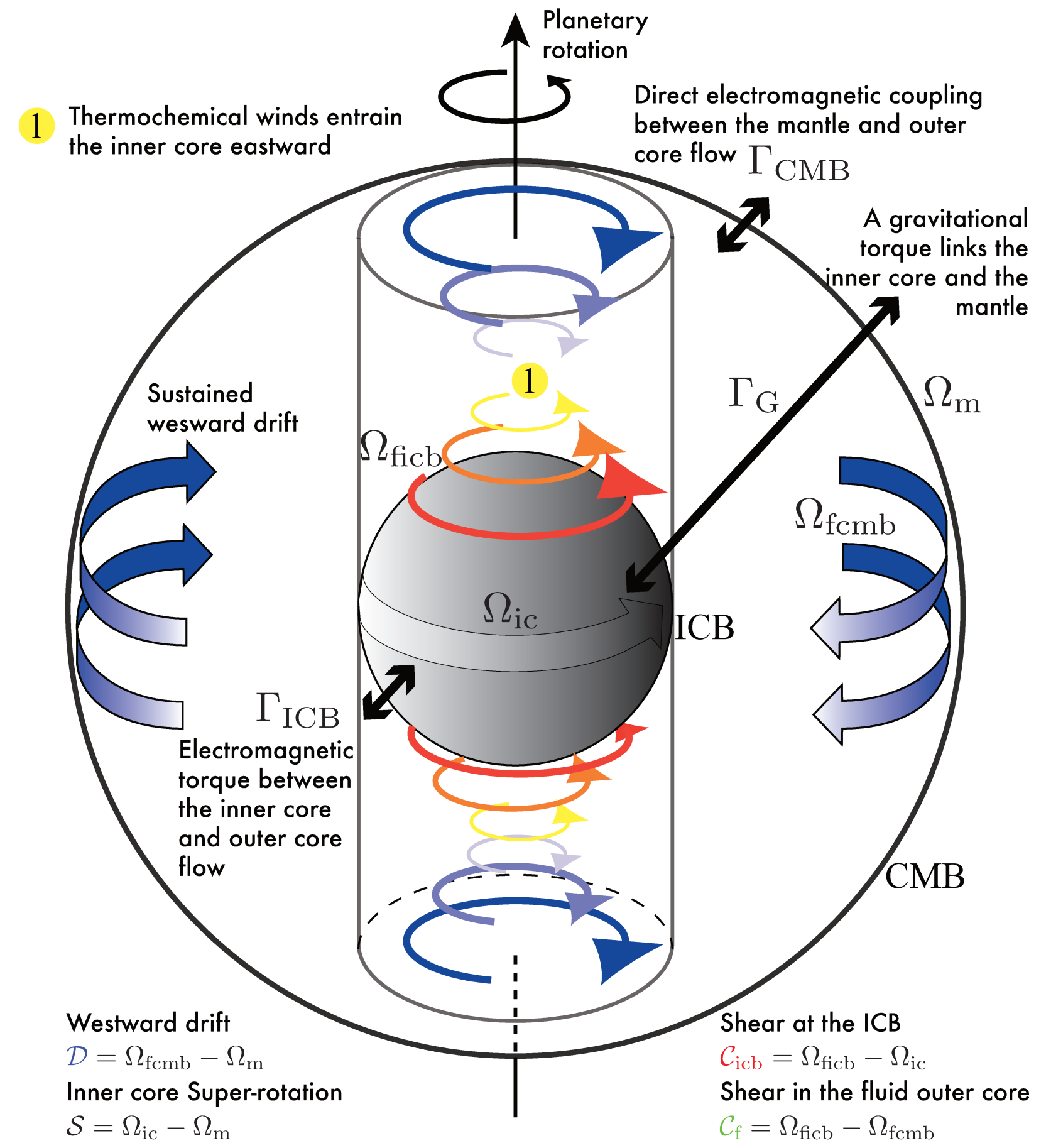

- Stress-free boundary conditions at both ICB and CMB

- Electrically conducting inner core and mantle (thin layer at the $\mathrm{CMB}$ )

Figure 1.20: Scheme of the system studied in this $\mathrm{PhD}$ work. The mantle and the inner core are free to axially rotate under the influence of two electromagnetic torque at ICB $\left(\Gamma_{I C B}\right)$ and $\mathrm{CMB}\left(\Gamma_{\mathrm{CMB}}\right)$ and a gravitational torque between the two $\left(\Gamma_{\mathrm{G}}\right)$. The angular velocities of the fluid core to the ICB and CMB are respectively $\Omega_{\mathrm{ficb}}$ and $\Omega_{\mathrm{fcmb}}$ while the solid body rotations of the inner core and the mantle are denoted by $\Omega_{\mathrm{ic}}$ and $\Omega_{\mathrm{m}}$. This introduce four typical shears: the geomagnetic westward drift $\mathcal{D}$, the shear at the ICB $\mathcal{C}_{\text {icb }}$, the shear in the fluid outer core $\mathcal{C}_{\mathrm{f}}$ and the inner core super-rotation $\mathcal{S}$. This scheme also pictures the tangent cylinder and the thermal wind flows described in section 1.5.1 named polar vortices. 


\begin{abstract}
This chapter establishes the equations governing convection of an incompressible, Newtonian and electrically conducting fluid in a rotating spherical shell, under the Boussinesq approximation. The induction equation is treated under the Magnetohydrodynamic (MHD) approximation. Chemical and compositional convection in the outer core are described through the formalism developed by Braginsky and Roberts (1995) while detailed treatement of the Boussinesq and anelastic fluid approximations follows that of Anufriev et al. (2005), complementary approaches that are neatly summarized in Jones (2015). Equations are then displayed in their dimensionless form, from which follows a discussion that compares convection in numerical geodynamo simulations to the Earth's core regime.
\end{abstract}

\title{
Résumé
}

Ce chapitre expose les équations qui régissent la convection d'un fluide conducteur, Newtonien et incompressible dans une coquille sphérique en rotation, soumis à l'approximation de Boussinesq. L'induction électromagnétique est traitée dans l'approximation de la magnétohydrodynamique (MHD). Les aspects chimiques et thermiques de la convection sont décrits en suivant le formalisme de Braginsky and Roberts (1995) et les détails des approximations de Boussinesq et de fluide anelastique sont tirés de Anufriev et al. (2005). Ces deux approches complémentaires sont d'ailleurs résumées par Jones (2015). Les équations sont ensuite présentées sous leur forme adimensionnée, donnant suite à une discussion comparant les régimes convectifs des simulations numériques de la géodynamo. 


\section{$2.1 \quad$ Geometry}

The fluid outer core is modeled as a spherical shell of length $D=r_{\mathrm{o}}-r_{\mathrm{i}}$, with $r_{\mathrm{i}}$ the radius of the inner core and $r_{\mathrm{o}}$ that of the fluid outer core. The system rotates at an angular velocity $\Omega=\Omega \cdot \mathbf{e}_{\mathrm{z}}$, with $\mathbf{e}_{\mathrm{z}}$ the unitary vector parallel to the rotation axis. The vector $\Omega$ is considered as invariant in space and over time. This set up thus ignores any precession or nutation phenomena, as well as any variations in the length of day (LOD) that do not originate in the core (see e.g. Gross, 2001, 2007). Fields are described with spherical coordinates $(r, \theta, \varphi)$, with $r$ the radius, $\theta$ the colatitude and $\varphi$ the longitude and $\left(\mathbf{e}_{\mathrm{r}}, \mathbf{e}_{\theta}, \mathbf{e}_{\varphi}\right)$ the associated basis.

\begin{tabular}{l|c|c|c}
\hline \hline \multicolumn{3}{|c}{ Core geometry } \\
\hline Notation & Name & Value & Unit \\
\hline \hline$r_{\mathrm{i}}$ & Inner core radius & $1.22 \times 10^{6}$ & $\mathrm{~m}$ \\
$r_{\mathrm{o}}$ & Outer core radius & $3.48 \times 10^{6}$ & $\mathrm{~m}$ \\
$D$ & Outer core thickness & $2.26 \times 10^{6}$ & $\mathrm{~m}$ \\
$\Omega$ & Angular velocity of rotation & $7.27 \times 10^{-5}$ & $\mathrm{rad} \mathrm{s}^{-1}$ \\
\hline \hline
\end{tabular}

Table 2.1: Geometry of the Earth's core from Dziewonski and Anderson (1981).

\subsection{Conservation laws}

The time rate of change of a scalar or vectorial quantity moving with an infinitesimal fluid parcel is described by its material or "Lagrangian" derivative

$$
\frac{D f}{D t}=\frac{\partial f}{\partial t}+\mathbf{u} \cdot \nabla f
$$

with the velocity field $\mathbf{u}\left(u_{\mathrm{r}}, u_{\theta}, u_{\varphi}\right)$ in spherical coordinates. This continuity equation reflects the fact that even is a steady flow $(\partial f / \partial t=0)$ the quantity $f$ may suffer changes as it moves with the flow.

\subsubsection{Mass}

The differential form of mass conservation, or continuity equation, is given by

$$
\frac{\partial \rho}{\partial t}+\nabla \cdot(\rho \mathbf{u})=0
$$

Our model describes liquid iron in the fluid outer core as an incompressible fluid of density $\rho=\rho_{0}$. The density being independent in space and time,

$$
\frac{D \rho}{D t}=0
$$


this implies from equation (2.2) that the velocity field is solenoidal ${ }^{1}$,

$$
\nabla \cdot \mathbf{u}=0
$$

\subsubsection{Momentum}

The momentum conservation of a fluid parcel is subject to surface stresses and volumetric forces,

$$
\rho \frac{D \mathbf{u}}{D t}=\nabla \cdot \Pi+\mathbf{f}_{\mathbf{b}}
$$

respectively embodied by the stress tensor $\Pi_{i j}$ and the volumetric body force term $\mathbf{f}_{\mathbf{b}}$. We will assume that liquid iron is a Newtonian incompressible fluid, so that viscous stresses are linearly proportional to the strain rate. In that case, the stress tensor can be written as

$$
\Pi_{i j}=-p \delta_{i j}+2 \mu \varepsilon_{i j}
$$

where $\delta_{i j}$ is the Kronecker delta symbol ${ }^{2}$ and $\varepsilon_{i j}$ is the strain-rate tensor. This expression also introduces the pressure $p$ and the dynamic viscosity of the fluid $\mu$, that will be considered as a constant in the following. Inserting equation (2.5) into equation (2.6) then leads to the incompressible Navier-Stokes equation

$$
\rho \frac{D \mathbf{u}}{D t}=-\nabla p+\rho \nu \nabla^{2} \mathbf{u}+\mathbf{f}_{\mathbf{b}}
$$

where we have introduced the kinematic viscosity $\nu=\mu / \rho$. This equation also assumes that the fluid is isotropic, i.e. its properties have no preferred direction, and is valid in a Newtonian or inertial reference frame (fixed or moving straight uniformly). In the following, we choose to place ourselves in the non-inertial rotating reference frame of constant angular velocity $\boldsymbol{\Omega}$. The transition between reference frame of a given vector field $\mathbf{v}$ is performed by

$$
\left.\mathbf{v}\right|_{\mathrm{I}}=\left.\mathbf{v}\right|_{\Omega}+\Omega \times \mathbf{r}
$$

where $\left.\mathbf{v}\right|_{\mathrm{I}}$ is the vector in the inertial reference frame and $\left.\mathbf{v}\right|_{\Omega}$ in the rotating frame, while its rate of change is given by

$$
\left.\frac{D \mathbf{v}}{D t}\right|_{\mathrm{I}}=\left.\frac{D \mathbf{v}}{D t}\right|_{\Omega}+\Omega \times \mathbf{v} .
$$

\footnotetext{
1. divergence-free vector field.

2. $\delta_{i j}=1$ if $i=j$ and $\delta_{i j}=0$ if $i \neq j$
} 
Hence, the Lagrangian derivative of $\left.\mathbf{u}\right|_{\mathrm{I}}$ in the inertial frame is

$$
\begin{aligned}
\left.\frac{\left.D \mathbf{u}\right|_{\mathrm{I}}}{D t}\right|_{\mathrm{I}} & =\left(\left.\frac{D}{D t}\right|_{\Omega}+\mathbf{\Omega} \times\right)\left(\left.\mathbf{u}\right|_{\Omega}+\boldsymbol{\Omega} \times \mathbf{r}\right) \\
& =\left.\frac{\left.D \mathbf{u}\right|_{\Omega}}{D t}\right|_{\Omega}+2 \Omega \times\left.\mathbf{u}\right|_{\Omega}+\boldsymbol{\Omega} \times \boldsymbol{\Omega} \times \mathbf{r} .
\end{aligned}
$$

We may then express the momentum conservation equation in the rotating frame, getting rid of the indices,

$$
\rho \frac{D \mathbf{u}}{D t}=-2 \rho \boldsymbol{\Omega} \times \mathbf{u}-\rho \boldsymbol{\Omega} \times \boldsymbol{\Omega} \times \mathbf{r}-\nabla P+\rho \nu \nabla^{2} \mathbf{u}+\mathbf{f}_{\mathbf{v}} .
$$

The change of reference frame introduces two new terms. The first one $(-2 \boldsymbol{\Omega} \times \mathbf{u})$ is the Coriolis acceleration that is directed "on the right" of the velocity vector if the rotation is counterclockwise, as for the Northern hemisphere of the Earth viewed from above the North pole. The second one $(-\Omega \times \Omega \times \mathbf{r})$ is named the centrifugal acceleration and is directed radially outward. As the latter may also be expressed as a gradient, it is often regrouped with the pressure gradient as

$$
-\nabla P=-\nabla p-\rho \boldsymbol{\Omega} \times \boldsymbol{\Omega} \times \mathbf{r} .
$$

\subsubsection{Angular momentum}

The angular momentum of the system is sensitive to two electromagnetic torques at the fluid core boundaries, $\Gamma_{\mathrm{ICB}}$ and $\Gamma_{\mathrm{CMB}}$. These torques represent the action of the Lorentz force (see Eq. 2.50) exerted on the inner core and the mantle, so that the general expression of an axial electromagnetic torque $\Gamma_{\mathrm{EM}}$ on a given electrically conducting volume $V$ is

$$
\Gamma_{\mathrm{EM}}=\mathbf{e}_{\mathrm{z}} \cdot \int_{V} \mathbf{r} \times \mathbf{J} \times \mathbf{B} \mathrm{dV} .
$$

As demonstrated by Rochester (1962) with the use of Maxwell magnetic stress tensor, this integral may be expressed by a surface integral for the product between the radial and azimuthal component of the magnetic field. At the inner core boundary, this gives

$$
\Gamma_{\mathrm{ICB}}=-\frac{r_{\mathrm{i}}}{\mu_{0}} \int_{S_{I C B}} B_{\mathrm{r}} B_{\varphi} \sin \theta \mathrm{dS}
$$

with $S_{I C B}$ the surface of the inner core boundary, and at the CMB we have

$$
\Gamma_{\mathrm{CMB}}=\frac{r_{\mathrm{o}}}{\mu_{0}} \int_{\mathrm{CMB}} B_{\mathrm{r}} B_{\varphi} \sin \theta \mathrm{dS} .
$$

with $S_{C M B}$ the surface of the core-mantle boundary. The mantle and the inner core are linked by a gravitational torque $\Gamma_{G}$ (Buffett, 1996a,b). This restoring torque will 
tend to minimize the misalignment angle $\phi$ between inner core gravitationally induced deformations and mantle density heterogeneities. The strength of this torque is given by the magnitude of mantle heterogeneities, embodied by the gravitational coupling constant $\Gamma$. The gravitational torque exerted by the mantle on the inner core is

$$
\Gamma_{\mathrm{G}}=-\phi \Gamma
$$

while the temporal evolution of the misalignment angle is a function of the solid body rotation of the inner core and the mantle, respectively $\Omega_{\mathrm{ic}}$ and $\Omega_{\mathrm{m}}$, and a viscous relaxation time scale of the inner core $\tau$ like

$$
\frac{\mathrm{d} \phi}{\mathrm{d} t}=\Omega_{\mathrm{ic}}-\Omega_{\mathrm{m}}-\frac{\phi}{\tau}
$$

By considering the above mentioned electromagnetic torques and the gravitational torque, the conservation of the angular momentum of the inner core, the mantle, and the fluid outer core is displayed by the following system,

$$
\begin{aligned}
\mathrm{I}_{\mathrm{i}} \frac{\mathrm{d} \Omega_{\mathrm{ic}}}{\mathrm{dt}} & =\Gamma_{\mathrm{G}}+\Gamma_{\mathrm{ICB}}, \\
\mathrm{I}_{\mathrm{m}} \frac{\mathrm{d} \Omega_{\mathrm{m}}}{\mathrm{dt}} & =-\Gamma_{\mathrm{G}}+\Gamma_{\mathrm{CMB}}, \\
\frac{\mathrm{d}}{\mathrm{dt}} \int_{V_{f}} \rho_{0}(r \sin \theta)^{2} \omega_{\mathrm{f}} \mathrm{d} V & =-\Gamma_{\mathrm{ICB}}-\Gamma_{\mathrm{CMB}},
\end{aligned}
$$

in which $I_{i}$ and $I_{m}$ are the moments of inertia of the inner core and the outer, considered as constants over time. Also, $V_{f}$ denotes the fluid outer core volume and $\omega_{\mathrm{f}}$ the angular velocity of rotation of a fluid parcel.

\subsubsection{Energy}

The conservation of energy is considered through the conservation of entropy $S$, an extensive state function. In the general case, the heat transport equation is given by

$$
\rho T \frac{D S}{D t}=\nabla \cdot\left(k_{T} \nabla T\right)+H_{T} \rho
$$

with $\mathrm{T}$ the temperature, $k_{T}$ the thermal conductivity of the fluid and $H_{T}$ the rate of heat release per unit mass, a given volumetric source term. As convection is supposed to be mainly driven by the release of light element at the ICB, the conservation of energy must also consider the composition equation, or transport equation, which is

$$
\frac{D \xi}{D t}=\nabla \cdot\left(k_{\xi} \nabla \xi\right)+H_{\xi},
$$


where $\xi$ is light elements concentration, $k_{\xi}$ the mass diffusion coefficient and $H_{\xi}$ a volumetric source term. Equations (2.20) and (2.21) described convection inside the core. However, the core is believed to be close to an adiabatic and well-mixed state, for which convection is driven by tiny fractions of density perturbation. Convection is therefore studied through deviations of the thermodynamic variables from a reference state. Thermodynamic quantities are then decomposed as

$$
p=p_{\mathrm{a}}+p^{\prime}, \quad T=T_{\mathrm{a}}+T^{\prime}, \quad \rho=\rho_{\mathrm{a}}+\rho^{\prime}, \quad \xi=\xi_{a}+\xi^{\prime}, \text { and } \quad S=S_{a}+S^{\prime},
$$

where the suffix a denotes adiabatic quantitates and the apostrophe their associated perturbations. The evolution of the perturbations over space and time is obtained by first considering the state equation of the entropy, assuming that variations of entropy are associated with variations in temperature, pressure and light element concentration like

$$
\begin{aligned}
\mathrm{d} S & =\left(\frac{\partial S}{\partial T}\right)_{p, \xi} \mathrm{d} T+\left(\frac{\partial S}{\partial p}\right)_{T, \xi} \mathrm{d} p+\left(\frac{\partial S}{\partial \xi}\right)_{p, T} \mathrm{~d} \xi \\
& =\frac{c_{\mathrm{p}}}{T} \mathrm{~d} T-\frac{\alpha_{T}}{\rho} \mathrm{d} p+\frac{h_{\xi}}{T} \mathrm{~d} \xi, .
\end{aligned}
$$

in which $c_{\mathrm{p}}$ is the heat capacity at constant pressure and $h_{\xi}$ the heat of reaction. Also, it is necessary to establish the reference state: the gradients of $p_{\mathrm{a}}, T_{\mathrm{a}}$ and $\rho_{\mathrm{a}}$ inside the core. The first simply express the hydrostatic pressure gradient like

$$
\frac{\mathrm{d} p_{\mathrm{a}}}{\mathrm{d} r}=-g \rho_{\mathrm{a}}
$$

As the adiabatic well-mixed reference state implies that entropy and light element concentration are constants (independent of position) inside the fluid outer core, we obtain the adiabatic temperature gradient from equations (2.24) and (2.25), we have

$$
\frac{\mathrm{d} T_{\mathrm{a}}}{\mathrm{d} r}=-\frac{\mathrm{D}_{\mathrm{i}} T_{\mathrm{a}}}{D}, \quad \text { with } \quad \mathrm{D}_{\mathrm{i}}=\frac{g \alpha_{T} D}{c_{\mathrm{p}}},
$$

the dissipation parameter. Then, we obtain the adiabatic density gradient from the expression of the Grüneinsen parameter, $\gamma=(\rho / T)(\partial T / \partial \rho)_{S, \xi}$, giving the relationship between density and temperature variations at constant entropy and light element concentration. This leads to

$$
\frac{\mathrm{d} \rho_{\mathrm{a}}}{\mathrm{d} r}=-\frac{-\mathrm{D}_{\mathrm{i}} \rho_{\mathrm{a}}}{\gamma D}
$$

Equations (2.25), (2.26) and (2.27) constitute the adiabatic well-mixed reference state. They may be used to obtain the evolution of perturbations in entropy from equation (2.20) like

$$
\rho_{\mathrm{a}} T_{\mathrm{a}} \frac{D S^{\prime}}{D t}=T_{\mathrm{a}} \boldsymbol{\nabla} \cdot\left(\frac{k_{T}}{c_{\mathrm{p}}} T_{\mathrm{a}} \boldsymbol{\nabla} S^{\prime}\right)+\nabla \cdot\left(k_{T} \boldsymbol{\nabla} T_{\mathrm{a}}\right)-\rho_{\mathrm{a}} T_{\mathrm{a}} \frac{\partial S_{\mathrm{a}}}{\partial t}+\rho_{\mathrm{a}} H
$$


and light elements perturbations from equation (2.21) like

$$
\frac{\partial \xi^{\prime}}{\partial t}+\mathbf{u} \cdot \nabla \xi^{\prime}=\frac{1}{\rho_{\mathrm{a}}} \boldsymbol{\nabla} \cdot\left(k_{\xi} \boldsymbol{\nabla} \xi^{\prime}\right)-\frac{\partial \xi_{\mathrm{a}}}{\partial t}
$$

where $H$ is a source/sink term gathering viscous and ohmic dissipation and heat release by radioctivity. These conservation equations will then be solved in the Boussinesq limit defined as $D_{i} \mapsto 0$. Though according to Dziewonski and Anderson (1981) the Earth's value of $D_{i}=$ $0.2-0.3$, it is often argue that such a value is sufficiently small to study tiny perturbations of thermodynamic quantities around the adiabat in the Boussinesq approximation. Moreover, this greatly simplify the equations and their numerical implementation, and offers the advantage of the comparison to laboratory experiments that are often in the Boussinesq limit. This limit may be view as

$$
\frac{c_{\mathrm{p}}}{g \alpha_{T}} \gg D
$$

where the term on the left hand-side corresponds to a characteristic length-scale of density variations. The Boussinesq approximation then neglects the effects adiabatic gradients on convection, the density variations in the momentum equation (except for the buoyancy force term) as well as the effects of viscous and ohmic dissipation in the entropy budget. Together with the anelastic liquid approximation $\left(\alpha_{T} T \ll 1\right)$ leading to entropy variations being mostly induced by temperature variation (see Anufriev et al., 2005),

$$
S^{\prime}=\frac{c_{\mathrm{p}}}{T_{\mathrm{a}}} T^{\prime}
$$

this limit allows the formulation of equations (2.28) and (2.29) like

$$
\frac{D T^{\prime}}{D t}=\kappa_{T} \nabla^{2} T^{\prime}+H^{*}
$$

with

$$
H^{*}=\frac{\boldsymbol{\nabla} \cdot\left(k_{T} \boldsymbol{\nabla} T_{\mathrm{a}}\right)}{\rho_{\mathrm{a}} c_{\mathrm{p}}}-\frac{T_{\mathrm{a}}}{c_{\mathrm{p}}} \frac{\partial S_{\mathrm{a}}}{\partial t},
$$

and

$$
\frac{\partial \xi^{\prime}}{\partial t}+\mathbf{u} \cdot \nabla \xi^{\prime}=k_{\xi} \nabla^{2} \xi^{\prime}-\frac{\partial \xi_{\mathrm{a}}}{\partial t}
$$

which are the Boussinesq form of the heat transport and light element transport equations, with $\kappa_{T}=k_{T} / \rho_{\mathrm{a}}$ the thermal diffusivity, and $\rho_{\mathrm{a}}=\rho_{0}$ a constant heraeafter. The first term of $H^{*}$ (Eq. 2.33) represents the heat conducted down the adiabat, corresponding to a sink term, while the second term embody the Earth's secular cooling. As $\partial S / \partial t<0$, it corresponds to a source term from the convection point of view. Because of the non-penetration condition at the CMB, the similar term in equation (2.34), $\partial \xi^{\prime} / \partial t$ is a volumetric sink term of light elements that balances the release of light element at the ICB. 


\subsubsection{Induction equation}

The induction equation governs the evolution of the magnetic field $\mathbf{B}$ in an electrically conducting fluid. It is obtained by a combination of the Maxwell's equations displayed below.

\section{Maxwell-Gauss}

The Maxwell-Gauss's law relates the electric field $\mathbf{E}$ to the electric charge density $\rho_{s}$ like

$$
\nabla \cdot \mathbf{E}=\frac{\rho_{s}}{\epsilon_{0}}
$$

with $\epsilon_{0}$ is the permittivity of free space.

\section{Maxwell-Faraday}

The Maxwell-Faraday's law indicates how an electric field can be induced by a variation of a magnetic field,

$$
\nabla \times \mathbf{E}=-\frac{\partial \mathbf{B}}{\partial t}
$$

\section{Maxwell-Thomson}

The Maxwell-Thomson's or "Maxwell-Flux" law states the non-existence of point source of magnetic field (magnetic monopole). The elementary object for the description of magnetic field is thus the magnetic dipole, implying that the magnetic field is a solenoidal field,

$$
\boldsymbol{\nabla} \cdot \mathbf{B}=0
$$

\section{Maxwell-Ampère}

The Maxwell-Ampère's law stipulates that a magnetic field may be induced by electric currents $\mathbf{J}$ or temporal variations of electric fields as

$$
\nabla \times \mathbf{B}=\mu_{0} \mathbf{J}+\mu_{0} \epsilon_{0} \frac{\partial \mathbf{E}}{\partial t}
$$

We will however use this law under the MHD approximation for which induction phenomenon occur at velocity $u$ much lower than the speed of light $c\left(u \ll c=1 / \sqrt{\mu_{0} \epsilon_{0}}\right)$, and neglect displacement currents. The Maxwell-Ampère's law is then reduces to

$$
\nabla \times \mathbf{B}=\mu_{0} \mathbf{J}
$$

Under this assumption, Ohm's law in an electrically conducting fluid with velocity field u is given by

$$
\mathbf{J}=\sigma(\mathbf{E}+\mathbf{u} \times \mathbf{B})
$$


with $\sigma$ the electrical conductivity of the fluid. Then, by inserting equation (2.40) into equation (2.36) we have

$$
\frac{\partial \mathbf{B}}{\partial t}=\boldsymbol{\nabla} \times(\mathbf{u} \times \mathbf{B})-\nabla \times(\eta \boldsymbol{\nabla} \times \mathbf{B}),
$$

in which $\eta=1 / \sigma \mu_{0}$ is the magnetic diffusivity. In the following, we will consider that the magnetic diffusivity of liquid iron is constant. Then, since the magnetic field is solenoidal (Eq. 2.37), equation (2.42) may be written

$$
\frac{\partial \mathbf{B}}{\partial t}=\boldsymbol{\nabla} \times(\mathbf{u} \times \mathbf{B})+\eta \boldsymbol{\nabla}^{2} \mathbf{B}
$$

This induction equation reflects that the temporal variability of the magnetic field is governed by the balance between advection (production of magnetic field through the term $\mathbf{u} \times \mathbf{B}$ ) and diffusion (term in $\nabla^{2} \mathbf{B}$ ), that is controlled by the fluid's magnetic diffusivity $\eta$.

\begin{tabular}{l|c|c|c}
\hline \hline \multicolumn{3}{c}{ Properties of liquid iron at core conditions } \\
\hline Notation & Name & Value & Unit \\
\hline \hline$\rho_{0}$ & Mean density & 11000 & $\mathrm{~kg} \mathrm{~m}^{-3}$ \\
$\mu$ & Dynamic viscosity & $10^{-3}$ & $\mathrm{~Pa} \mathrm{~s}$ \\
$\nu$ & Kinematic viscosity & $10^{-6}$ & $\mathrm{~m}^{2} \mathrm{~s}^{-1}$ \\
$\kappa$ & Thermal diffusivity & $5 \times 10^{-6}$ & $\mathrm{~m}^{2} \mathrm{~s}^{-1}$ \\
$\sigma$ & Electrical conductivity & $1.5 \times 10^{6}$ & $\mathrm{~S} \mathrm{~m}^{-1}$ \\
$\eta$ & Magnetic diffusivity & 0.5 & $\mathrm{~m}^{2} \mathrm{~s}^{-1}$ \\
\hline \hline
\end{tabular}

Table 2.2: Properties of liquid iron at core conditions from Dziewonski and Anderson (1981), Pozzo et al. (2012) and de Wijs et al. (1998).

\subsubsection{Dimensioned system}

In order to obtain the complete expression of the Navier-Stokes equation for our system we now need to incorporate the different volumetric body forces that will act on the fluid.

\section{Buoyancy force}

Following Anufriev et al. (2005), the density perturbation $\rho^{\prime}$ are induced by entropy, pressure and composition perturbations like

$$
\begin{aligned}
\rho^{\prime} & =S^{\prime}\left(\frac{\partial \rho}{\partial S}\right)_{p, \xi}+p^{\prime}\left(\frac{\partial \rho}{\partial p}\right)_{S, \xi}+\xi^{\prime}\left(\frac{\partial \rho}{\partial \xi}\right)_{p, S}, \\
& =-\frac{\rho_{\mathrm{a}} \alpha_{T} T_{\mathrm{a}}}{c_{\mathrm{p}}} S^{\prime}-\frac{p^{\prime}}{g \rho_{\mathrm{a}}} \frac{\mathrm{d} \rho_{\mathrm{a}}}{\mathrm{d} r}-\rho_{\mathrm{a}} \alpha_{\xi} \xi^{\prime}
\end{aligned}
$$


with $\alpha_{\xi}$ the adiabatic compositional expansion coefficient. In the Boussinesq limit developed in section 2.2.4, and using $S^{\prime}=c_{\mathrm{p}} T^{\prime} / T_{\mathrm{a}}$ and $\rho_{\mathrm{a}}=\rho_{0}$, the density perturbations reduces to

$$
\rho^{\prime}=-\rho_{0}\left(\alpha_{T} T^{\prime}+\alpha_{\xi} \xi^{\prime}\right)
$$

Any perturbation $p^{\prime}$ in the fluid outer core will produce a Buoyancy force $\mathbf{f}_{\mathrm{b}}$ on the fluid parcel that is proportional to

$$
\mathbf{f}_{\mathbf{b}}=\rho^{\prime} \mathbf{g}
$$

with $\mathbf{g}$ is the gravity acceleration. The gravity acceleration is supposed to vary linearly with depth in the fluid outer core such that $\mathbf{g}=-g_{0} \mathbf{r} / r_{\mathrm{o}}$, with $g_{0}$ the gravity value at the core-mantle boundary. To described both thermal and compositional effect of the buoyancy force, we follow the formalism of Braginsky and Roberts (1995) and introduce the codensity $C$. Here, the codensity is simplified to

$$
C=\rho_{0}\left(\alpha_{T} T^{\prime}+\beta \xi^{\prime}\right)
$$

considering that $\alpha_{\xi}=\beta / \rho_{0}$, with $\beta$ the density difference between liquid iron and light elements in the core. The system is then described by an effective thermochemical diffusivity $\kappa$ by assuming that turbulent convection in the core bring the temperature and light elements field to the same diffusivity. The transport equation thus becomes

$$
\frac{D C}{D t}=\kappa \nabla^{2} C+S_{T / \xi}^{\prime}
$$

in which $S_{T / \xi}^{\prime}$ represents the sources and sinks of codensity (Aubert et al., 2009), ensuring the mass conservation in the core.

\section{Lorentz force}

Motions of an electrically conducting fluid embedded in a magnetic field $\mathbf{B}$ generates electric currents that will oppose the change that induced them, according to Lenz's law. Such electric currents $\mathbf{J}$ may be related to the curl of the magnetic field by means of Ampère's law

$$
\mu_{0} \mathbf{J}=\nabla \times \mathbf{B}
$$

with $\mu_{0}$ the permeability of free space. The induced magnetic field will exert a feedback on the flow, named the Lorentz force $\mathbf{f}_{\mathbf{l}}$ that can be expressed as

$$
\mathbf{f}_{\mathbf{l}}=\mathbf{J} \times \mathbf{B}=\frac{1}{\mu_{0}} \nabla \times \mathbf{B} \times \mathbf{B} .
$$

Inserting the Buoyancy and Lorentz forces into the momentum equation (Eq. 2.12) leads to the complete expression of the momentum conservation of liquid iron in the outer core, which describes the motion of an electrically conducting fluid in a rotating 
spherical shell under the Boussinesq approximation. Though we kept the same notation, the pressure gradient $\nabla P$ is now an "effective" pressure gradient arising from the introduction of $P=P_{\mathrm{a}}+P^{\prime}$ and the consideration that buoyancy forces associated to pressure perturbations do not contribute. Equations (2.4), (2.12) with buoyancy $\mathbf{f}_{\mathbf{b}}$ and Lorentz forces $\mathbf{f}_{\mathbf{l}},(2.48)$, (2.37) and (2.42) form the basic system of MHD equations in the Boussinesq approximation, that are numerically solved in geodynamo models. This system is resumed as

$$
\begin{aligned}
\boldsymbol{\nabla} \cdot \mathbf{u} & =0, \\
\frac{\partial \mathbf{u}}{\partial t}+\mathbf{u} \cdot \boldsymbol{\nabla} \mathbf{u}+2 \boldsymbol{\Omega} \times \mathbf{u} & =-\frac{\boldsymbol{\nabla} P}{\rho_{0}}+\nu \boldsymbol{\nabla}^{2} \mathbf{u}+\mathbf{g} C+\frac{1}{\rho_{0} \mu_{0}} \boldsymbol{\nabla} \times \mathbf{B} \times \mathbf{B}, \\
\frac{\partial C}{\partial t}+\mathbf{u} \cdot \boldsymbol{\nabla C} & =\kappa \boldsymbol{\nabla}^{2} C+S_{T / \xi}^{\prime}, \\
\boldsymbol{\nabla} \cdot \mathbf{B} & =0, \\
\frac{\partial \mathbf{B}}{\partial t} & =\boldsymbol{\nabla} \times(\mathbf{u} \times \mathbf{B})+\eta \boldsymbol{\nabla}^{2} \mathbf{B},
\end{aligned}
$$

in which we incorporated the codensity formalism developed in section 2.2.4.

\subsubsection{Boundary conditions}

The set of equations displayed in the above section 2.2.6 is subject to boundary conditions on the velocity field, the magnetic field and codensity field.

\section{Mechanical conditions}

Though the relevant condition on the velocity field is that of no-slip $\mathbf{u}\left(r_{\mathrm{i}}, \theta, \varphi\right)=\mathbf{u}\left(r_{\mathrm{i}}, \theta, \varphi\right)=$ 0 at both ICB and CMB, we adopt stress free boundary conditions at the fluid core boundaries. This strongly mitigates the influence of viscosity, that is overestimated by several order of magnitude (see section 2.3.4). The velocity field is still subject to a no penetration condition at both ICB and CMB, the radial component of $\mathbf{u}$ is thus set to zero. These conditions can be written

$$
\begin{aligned}
u_{\mathrm{r}} & =0, \\
\frac{\partial}{\partial r}\left(\frac{u_{\theta}}{r}\right) & =0, \\
\frac{\partial}{\partial r}\left(\frac{u_{\varphi}}{r}\right) & =0,
\end{aligned}
$$

at $\operatorname{ICB}\left(r=r_{\mathrm{i}}\right)$ and $\mathrm{CMB}\left(r=r_{\mathrm{o}}\right)$.

\section{Magnetic conditions}

As the inner core is considered as electrically conducting, the inner core boundary is not a boundary for the magnetic field. This is also the case at the CMB due to the presence 
of an electrically conducting layer at the base of the mantle, of thickness $\Delta$. Beyond this layer, the mantle is considered as insulating, implying that the magnetic field is a potential field. This can be written,

$\forall r \in\left[r_{\mathrm{o}}+\Delta ; \infty[\right.$

$$
\mathbf{B}=-\nabla \Phi
$$

with,

$$
\nabla^{2} \Phi=0
$$

\section{Thermochemical conditions}

We adopt fixed-flux boundary conditions at both ICB and CMB. If not stipulate, the mass anomaly flux is spatially homogeneous and constant over time at the ICB,

$$
F=\int_{S_{\mathrm{ICB}}} \kappa \nabla C \cdot \mathrm{dS}
$$

and is set to zero at the CMB,

$$
0=\int_{S_{\mathrm{CMB}}} \kappa \nabla C \cdot \mathrm{dS}
$$

with $S_{\mathrm{CMB}}$ and $S_{\mathrm{CMB}}$ the surface of the inner core boundary and the core mantle boundary, respectively. In such a situation, the convection in entirely driven by the flux at the ICB, while the mass conservation is ensured by a volumetric sink term of codensity which is present in equation (2.32).

\subsection{Dimensionless equations}

In fluid dynamics, and more broadly in physics, it is convenient to deal with dimensionless variables. The transition from dimensioned to dimensionless variables is performed through an identification of the characteristic dimensions of the system. This technique leads to a parametrization of the equations that is more appropriate for the numerical implementation and identifies the force balances they involve. It is submitted to the Vaschy-Buckingham theorem (or Buckingham $\pi$ theorem) which states that an equation may be written in term of $p=n-k$ dimensionless parameters, with $n$ the number of physical variables and $k$ the number of physical dimensions involved.

\subsubsection{Viscous scaling}

The classical viscous scaling adopts $D^{2} / \nu$ as a characteristic time scale, with typical length scale $D$, the thickness of the outer core. This leads to a characteristic velocity $\nu / D$ and pressure $\rho \nu \Omega$, while the characteristic codensity is chosen as $[C]$. The scale of magnetic 
field is obtained by considering that the Elsasser number $\Lambda$, the ratio between Coriolis and Lorentz forces is close to unity in the fluid outer core,

$$
\Lambda=\frac{\sigma B^{2}}{\rho_{0} \Omega} \approx 1
$$

so that a characteristic $B$ scale is $\left(\rho_{0} \Omega / \sigma\right)^{\frac{1}{2}}$, or $\left(\mu_{0} \eta \rho_{0} \Omega\right)^{\frac{1}{2}}$ equivalently. In that case, the set of dimensioned equations defined in section 2.2.6 may be expressed as

$$
\begin{aligned}
\boldsymbol{\nabla} \cdot \mathbf{u} & =0, \\
E\left(\frac{\partial \mathbf{u}}{\partial t}+\mathbf{u} \cdot \nabla \mathbf{u}\right)+2 \mathbf{e}_{\mathrm{z}} \times \mathbf{u} & =-\boldsymbol{\nabla} P+E \boldsymbol{\nabla}^{2} \mathbf{u}+\mathcal{R} a \frac{\mathbf{r}}{r_{\mathrm{o}}} C+\frac{1}{P m} \boldsymbol{\nabla} \times \mathbf{B} \times \mathbf{B}, \\
\frac{\partial C}{\partial t}+\mathbf{u} \cdot \boldsymbol{\nabla C} & =\frac{1}{P r} \boldsymbol{\nabla}^{2} C+S_{T / \xi}^{\prime}, \\
\boldsymbol{\nabla} \cdot \mathbf{B} & =0, \\
\frac{\partial \mathbf{B}}{\partial t} & =\boldsymbol{\nabla} \times(\mathbf{u} \times \mathbf{B})+\frac{1}{P m} \nabla^{2} \mathbf{B},
\end{aligned}
$$

in which we kept the same notation for the dimensionless quantities. This system is governed by four dimensionless numbers, the Ekman number

$$
E=\frac{\nu}{\Omega D^{2}}
$$

that measures the relative importance between viscous and Coriolis forces, the Prandtl number number

$$
\operatorname{Pr}=\frac{\nu}{\kappa}
$$

the ratio between viscous and thermochemical diffusivities, the magnetic Prandtl number

$$
P m=\frac{\nu}{\eta}
$$

the ratio between viscous and magnetic diffusivities, and the Rayleigh number,

$$
\mathcal{R} a=\frac{g_{0}[C] D}{\Omega \nu}
$$

that measures the vigor of convection in the fluid outer core. Though this is not our preferred way to obtain the dimensionless system, this introduces the four basic dimensionless numbers and exposes the equations as they are implemented in the PARODY-JA code (Dormy et al., 1998; Aubert et al., 2008).

\subsubsection{Diffusion-free scaling}

This scaling is based on the inverse of the Earth's angular velocity of rotation $\Omega^{-1}$ as a typical time scale. The thickness of the spherical shell is kept as typical length 
scale, dymamic pressure is rescaled with $\rho_{0} \Omega^{2} D^{2}$, the magnetic field as $\left(\sigma \mu_{0}\right)^{\frac{1}{2}} \Omega D$ and the codensity with $F / 4 \pi \Omega D^{3}$, with $F$ the mass anomaly flux at the inner core boundary, considered as spatially homogeneous and constant over time (Aubert et al., 2009). The dimensionless system then become

$$
\begin{aligned}
\boldsymbol{\nabla} \cdot \mathbf{u} & =0, \\
\frac{\partial \mathbf{u}}{\partial t}+\mathbf{u} \cdot \boldsymbol{\nabla} \mathbf{u}+2 \mathbf{e}_{\mathrm{z}} \times \mathbf{u} & =-\boldsymbol{\nabla} P+E \boldsymbol{\nabla}^{2} \mathbf{u}+\mathcal{R} a_{F} \frac{\mathbf{r}}{r_{\mathrm{o}}} C+\frac{1}{P m} \boldsymbol{\nabla} \times \mathbf{B} \times \mathbf{B}, \\
\frac{\partial C}{\partial t}+\mathbf{u} \cdot \boldsymbol{\nabla C} & =E_{\kappa} \boldsymbol{\nabla}^{2} C+S_{T / \xi}^{\prime}, \\
\boldsymbol{\nabla} \cdot \mathbf{B} & =0, \\
\frac{\partial \mathbf{B}}{\partial t} & =\boldsymbol{\nabla} \times(\mathbf{u} \times \mathbf{B})+E_{\eta} \nabla^{2} \mathbf{B},
\end{aligned}
$$

in which we introduced the thermochemical Ekman number

$$
E_{\kappa}=\frac{E}{P r}=\frac{\kappa}{\Omega D^{2}},
$$

in which we introduced the magnetic Ekman number

$$
E_{\eta}=\frac{E}{P m}=\frac{\eta}{\Omega D^{2}}
$$

and the flux Rayleigh number

$$
\mathcal{R} a_{F}=\frac{g_{0} F}{\rho \Omega^{3} D^{4}} .
$$

This choice of characteristic dimensions was initiated by Christensen and Aubert (2006) in order to derive scaling laws for the Earth's core regime that do not depend on magnetic or viscous diffusivities. Though it may be considered as our default choice of characteristic dimensions in this work, our ambition is not to support, nor oppose, the arguments developed in the study of Christensen and Aubert (2006). Indeed, it was at first more convenient to work with the same dimensionless variables as in the reference model (Aubert et al., 2013) and, we also consider this choice as more relevant to describe the long-term dynamics of the core in terms of typical shears in Pichon et al. (2016).

\subsubsection{Core regime}

The core regime is characterized by the value of the dimensionless parameters exposed in the previous section. From tables 2.1 and 2.2, we find that the Ekman number is of order $10^{-15}$, showing the dominance of the Coriolis force. The smallness of the thermochemical and magnetic Prantdl number $\left(P r \simeq 0.1\right.$ and $\left.P m \simeq 10^{-6}\right)$ also suggest that the loss of energy is mostly dominated by Ohmic dissipation. Typical Rayleigh number values may be found in Gubbins (2001), for thermal and compositional convection, which are at least $10^{6}$ times greater than the critical Rayleigh number for magnetoconvection, expressing the 
vigor of convection in the Earth's core. The values of the above dimensionless parameters are summurized in table 2.3, which also includes three additional parameters estimated by core flow inversions. They are obtained by introducing a characteristic velocity in the equation $\mathcal{U}$, which is the root-mean-square velocity of outer core flow close to the CMB. The first one is the Rossby number

$$
R o=\frac{\mathcal{U}}{\Omega D}
$$

and expresses the relative importance between the Coriolis and inertial forces. Also, the Reynolds number

$$
R e=\frac{\mathcal{U} D}{\nu}
$$

and the magnetic Reynolds number,

$$
R m=\frac{\mathcal{U} D}{\eta}
$$

measure the ratio between advection and viscous or Ohmic dissipation, respectively.

\begin{tabular}{l|c|c|c}
\hline \hline \multicolumn{4}{|c}{ Core regime } \\
\hline Notation & Name & Expression & Value \\
\hline \hline$E$ & Ekman & $\nu / \Omega D^{2}$ & $10^{-15}$ \\
$P r$ & Prandtl & $\nu / \kappa$ & $10^{-1}$ \\
$P m$ & Magnetic Prandtl & $\nu / \eta$ & $10^{-6}$ \\
$\mathcal{R} a_{F}$ & Flux Rayleigh & $g_{0} F / \rho \Omega^{3} D^{4}$ & $10^{-12}$ \\
$R o$ & Rossby & $\mathcal{U} / \Omega D$ & $10^{-6}$ \\
$R e$ & Reynolds & $\mathcal{U} D / \nu$ & $10^{9}$ \\
$R m$ & Magnetic Reynolds & $\mathcal{U} D / \eta$ & $10^{3}$ \\
\hline \hline
\end{tabular}

Table 2.3: Dimensionless parameters characterizing the fluid outer core flow.

\subsubsection{Geodynamo simulation regime}

Table 2.4 exposes the main input and output parameters of the Coupled-Earth (CE) model of Aubert et al. (2013). This numerical simulation, and geodynamo simulation in general, operate at Ekman numbers that are several orders of magnitude than the inferred Ekman number of the Earth's core, meaning that viscosity is largely overestimated. This is however not a conscious choice, since the Ekman value is directly constrained by the computing power available. Indeed, such a small Ekman value in the Earth's core imply that very small scale turbulence should be resolved, leading to massive computing costs. This effect may also be seen in the Ekman layer: characteristic flow pattern observed close to the boundaries in presence of rotation (see e.g. Dormy et al., 1998). As the thickness of the Ekman layer scales as the square root of the Ekman number, such a layer in the core 
must have a thickness on the order of ten centimeters, implying a resolution requirement that may not be reached yet.

\begin{tabular}{l|c|c|c|c|c|c|c|c|c}
\hline \hline \multicolumn{10}{c}{ Geodynamo simulations regime } \\
\hline Code & $\mathrm{E}$ & $\mathcal{R} a_{F}$ & $\mathrm{Pr}$ & $\mathrm{Pm}$ & $\mathrm{Ro}$ & $\mathrm{Re}$ & $\mathrm{Rm}$ & $\mathrm{NR}$ & $L_{\max }$ \\
\hline \hline $\mathrm{CE}$ & $3 \times 10^{-5}$ & $2.7 \times 10^{-5}$ & 1 & 2.5 & $10^{-2}$ & 377 & 943 & 160 & 133 \\
$\mathrm{CE} / \mathrm{L}$ & $10^{-8}$ & $9 \times 10^{-9}$ & 1 & $4.5 \times 10^{-2}$ & $2.4 \times 10^{-4}$ & $2.4 \times 10^{4}$ & 1082 & 624 & 133 \\
$\mathrm{~S} 2$ & $10^{-7}$ & $\simeq 10^{-8}$ & 1 & 0.1 & $5.1 \times 10^{-4}$ & 5140 & 514 & 1280 & 1000 \\
\hline \hline
\end{tabular}

Table 2.4: Dimensionless input and output parameters of geodynamo simulations of the CE model from Aubert et al. (2013), the reference model in this work. A comparison can be maybe with two recent simulations, the model $0 \mathrm{CE} / \mathrm{L}$ simulation of Aubert et al. (2017) with the highest radial resolution (NR) and the S2 simulation from Schaeffer et al. (2017).

However, as demonstrated in Introduction section 1.6.1, the CE model succeeds in reproducing the main features of the magnetic secular variation. The natural question that flow from this observation is then "why ?", given the gap between Earth's parameters and geodynamo simulations. The answer may lie in the capacity of geodynamo simulations to reach Earth-like values of the magnetic Reynolds number (Eq. 2.83), and potentially the right force balance to reproduce the large-scale dynamics of the Earth's core, for which the smallness of the Ekman number may have lower impacts. The Earth-likeness of geodynamo simulations is therefore proved to be bounded by values of the magnetic Reynolds number and magnetic Ekman number by Christensen et al. (2010). It is embodied by the parameter $\chi^{2}$, a measurement of the match between the Earth's magnetic field that from numerical simulations in terms dipolarity, symmetry, zonality and flux concentration at the CMB.

Table 2.4 also displays the input and output parameters of two recent geodynamo simulation. The first one is the model $0 \mathrm{CE} / \mathrm{L}$ simulation of Aubert et al. (2017), which is a large-eddy simulation based on the CE model (Aubert et al., 2013). In order to reach parameters values approaching the core values, Aubert et al. (2017) established a unidimensional path between standard simulations and core conditions. Small Ekman values are reached by using hyperdiffusivity, that suppresses small scale turbulence. However, the large-scale flow is found to be unaffected and invariant along the path. Since this path preserves the value of the magnetic Reynolds number, this result demonstrates that standard geodynamo simulations are relevant to account for the large-scale dynamics of the Earth's core. This contrast with the results of Schaeffer et al. (2017), exposed for the simulation $\mathrm{S} 2$ in table 2.4. This simulation represents the best attempt to reach the core regime, notably in terms of Ekman number (no hyperdiffusivity was used) and is also the more costly numerical simulation of the geodynamo at the time, involving 8192 cores. The radial (NR) and lateral resolution (by the maximum degree of the spherical harmonic expansion $L_{\max }$ ) are then one order of magnitude greater than the CE model. In many aspects this simulation is the closest simulation to the Earth's core, but is still considered by the authors as not having reached the exact core regime. 
As a conclusion, though geodynamo simulations do not operate at the regime of the core, they succeed in reproducing the large-scale dynamics and the main features of the magnetic field, for which the smallness of the Ekman number is less crucial. They are limited by the computational power available, and most recent dynamo simulation run at Ekman numbers that are still seven orders of magnitude too high. If Moore's law ${ }^{3}$ continues to be verified in the future, the computational power should hopefully no longer remain a limiting factor in the next few decades.

3. An empirical law of Gordon Moore, who stated in 1965 that the number of transistors in integrated circuits should double every two years (modified to 18 months), an exponential growth which may be related to computational power. 


\title{
Numerical implementation
}

\begin{abstract}
This chapter presents the numerical implementation of this study. It displays the Toroidal-Poloidal decomposition of the vector fields, the spherical harmonic expansion as well as the radial and temporal schemes in section 3.1 as further developed in Dormy (1997). Section 3.2 is dedicated to few improvements to the code in terms of boundary conditions at the core-mantle boundary and the distinction between advective and diffusive ("leakage") components of the electromagnetic torque acting on the mantle, as well as a time-dependent integration of the gravitational torque between the inner core and the mantle.
\end{abstract}

\section{Résumé}

Ce chapitre résume tout d'abord l'implémentation numérique des équations présentées dans le chapitre précédent, établie par Dormy (1997). Les champs vectoriels son décomposés en scalaires poloïdaux et toroïdaux, eux-mêmes étendus sur la base des harmoniques sphériques. Il décrit également le schéma d'integration radial en différences finies centrées ainsi que le schéma temporel semi-implicite, Crank-Nicolson pour les termes de diffusion et Adams-Bashforth pour les termes non-linéaires. La seconde partie présente les améliorations apportées au code. L'implémentation exacte des conditions magnétiques à la frontière noyau-manteau permet alors la décomposition du couple électromagnétique en ses parties diffusive et advective. Le couplage gravitationnel entre le noyau et le manteau est également implémenté pour mieux rendre compte des variations temporelles de la rotation de la graine, autorisant alors l'étude des influences respectives des hétérogénéités de densité dans le manteau et de la viscosité de la graine. 


\subsection{Parody-JA Code}

The Parody-JA code is a branching of the Parody code, developed by Emmanuel Dormy and Julien Aubert to solve the MHD equations in a spherical rotating shell. The original source code may be acquired upon request to Julien Aubert ${ }^{1}$.

\subsubsection{Toroidal-Poloidal decomposition}

Any solenoidal ${ }^{2}$ vector field $\mathbf{V}$ can be decomposed into poloidal and toroidal scalars, respectively $V_{\mathrm{p}}$ and $V_{\mathrm{t}}$, like

$$
\mathrm{V}=\boldsymbol{\nabla} \times \nabla \times \mathbf{r} V_{\mathrm{p}}+\nabla \times \mathbf{r} V_{\mathrm{t}}
$$

with $\mathbf{r}=r \mathbf{e}_{\mathrm{r}}$ the radius vector . This decomposition is unique and allows the determination of the three components of a given vector by two scalars. It is thus of major interest of the numerical implementation of the solenoidal velocity $\mathbf{u}$ and magnetic field $\mathbf{B}$ in our system. This decomposition, also referred to as "Mie decomposition", may be expressed as

$$
\mathbf{V}=\left(\begin{array}{c}
V_{\mathrm{r}} \\
V_{\theta} \\
V_{\varphi}
\end{array}\right)=\left(\begin{array}{c}
\frac{1}{r} L_{2} V_{\mathrm{p}} \\
\frac{\partial}{\partial \theta}\left(\frac{1}{r} \frac{\partial}{\partial r}\left(r V_{\mathrm{p}}\right)\right)+\frac{1}{\sin \theta} \frac{\partial V_{\mathrm{t}}}{\partial \varphi} \\
\frac{1}{\sin \theta} \frac{\partial}{\partial \varphi}\left(\frac{1}{r} \frac{\partial}{\partial r}\left(r V_{\mathrm{p}}\right)\right)-\frac{\partial V_{\mathrm{t}}}{\partial \theta}
\end{array}\right)
$$

Also, it is possible to demonstrated that the scalar product between the radius vector and the vector $\mathbf{V}$ involves the Laplace-Beltrami operator $L_{2}$ like,

$$
\mathbf{V} \cdot \mathbf{r}=L_{2} V_{\mathrm{p}}
$$

while the same scalar product with the rotational of $\mathbf{V}$ is leads to,

$$
(\boldsymbol{\nabla} \times \mathbf{V}) \cdot \mathbf{r}=L_{2} V_{\mathrm{t}}
$$

The $L_{2}$ operator, also named horizontal Laplacian, is defined as

$$
\begin{aligned}
L_{2} & =\frac{\partial}{\partial r} r^{2} \frac{\partial}{\partial r}-r^{2} \nabla^{2} \\
& =-\frac{1}{\sin \theta} \frac{\partial}{\partial \theta} \sin \theta \frac{\partial}{\partial \theta}-\frac{1}{\sin ^{2} \theta} \frac{\partial^{2}}{\partial \varphi^{2}}
\end{aligned}
$$

1. aubert@ipgp.fr

2. Divergence free field, $\boldsymbol{\nabla} \cdot \mathbf{V}=0$. 
and represents the lateral derivatives of the laplacian operator $\nabla^{2}$. This decomposition may be extended to non solenoidal fields, which is useful to compute the advection term $(\mathbf{u} \times \mathbf{B})$ which are not necessarily divergence free. In such a case, one may introduce a spheroidal scalar $V_{\mathrm{s}}$ to replace the poloidal scalar in the decomposition (Eq. 3.2). For a solenoidal field the relationship between the two is given by

$$
V_{\mathrm{s}}=\frac{1}{r} \frac{\partial}{\partial r}\left(r V_{\mathrm{p}}\right)
$$

The introduction of this decomposition into the dimensionless system (2.65)-(2.68) presented in the previous chapter leads, for example, to a decomposition of the induction equation into two scalar equations like

$$
\left(\frac{\partial}{\partial t}-\frac{\nabla^{2}}{P m}\right) L_{2} B_{\mathrm{p}}=\mathbf{r} \cdot(\boldsymbol{\nabla} \times \mathbf{u} \times \mathbf{B})
$$

and

$$
\left(\frac{\partial}{\partial t}-\frac{\nabla^{2}}{P m}\right) L_{2} B_{\mathrm{t}}=\mathbf{r} \cdot(\boldsymbol{\nabla} \times(\boldsymbol{\nabla} \times \mathbf{u} \times \mathbf{B})),
$$

where $B_{\mathrm{p}}$ and $B_{\mathrm{r}}$ are the poloidal and toroidal scalars, respectively.

\subsubsection{Radial discretization}

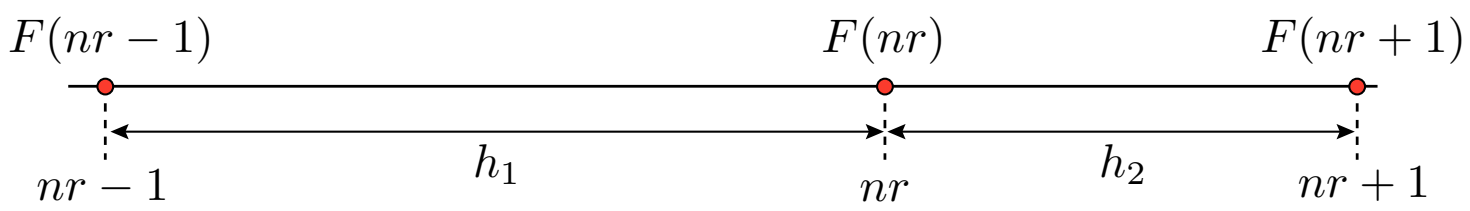

The radial implementation is performed by a centered finite difference scheme, on an irregular grid. The first radial derivative of a given function $F$ at point $n r$ is then evaluated by

$$
\partial F(n r)=\frac{h_{1}}{h_{1}+h_{2}}\left(\frac{F(n r+1)-F(n r)}{h_{2}}\right)+\frac{h_{2}}{h_{1}+h_{2}}\left(\frac{F(n r)-F(n r-1)}{h_{1}}\right),
$$

while the second radial derivative is evaluated by

$$
\partial^{2} F(n r)=\frac{h_{2} F(n r-1)-\left(h_{1}+h_{2}\right) F(n r)+h_{1} F(n r+1)}{h_{1} h_{2}\left(h_{1}+h_{2}\right) / 2} .
$$

The irregularity of the grid is controlled by the ratio between the number of regular and irregular intervals (Ratio1) and the ratio between the minimum and maximum interval $h$ (Ratio2). This irregularity is presented in figure 3.1, an expresses a refined mesh grid 
close to the fluid core boundaries as well as a constant mesh grid in the bulk of the outer core. Due to irregularity, introducing terms in $\left(h_{2}-h_{1}\right)$ in Taylor's development, it is not

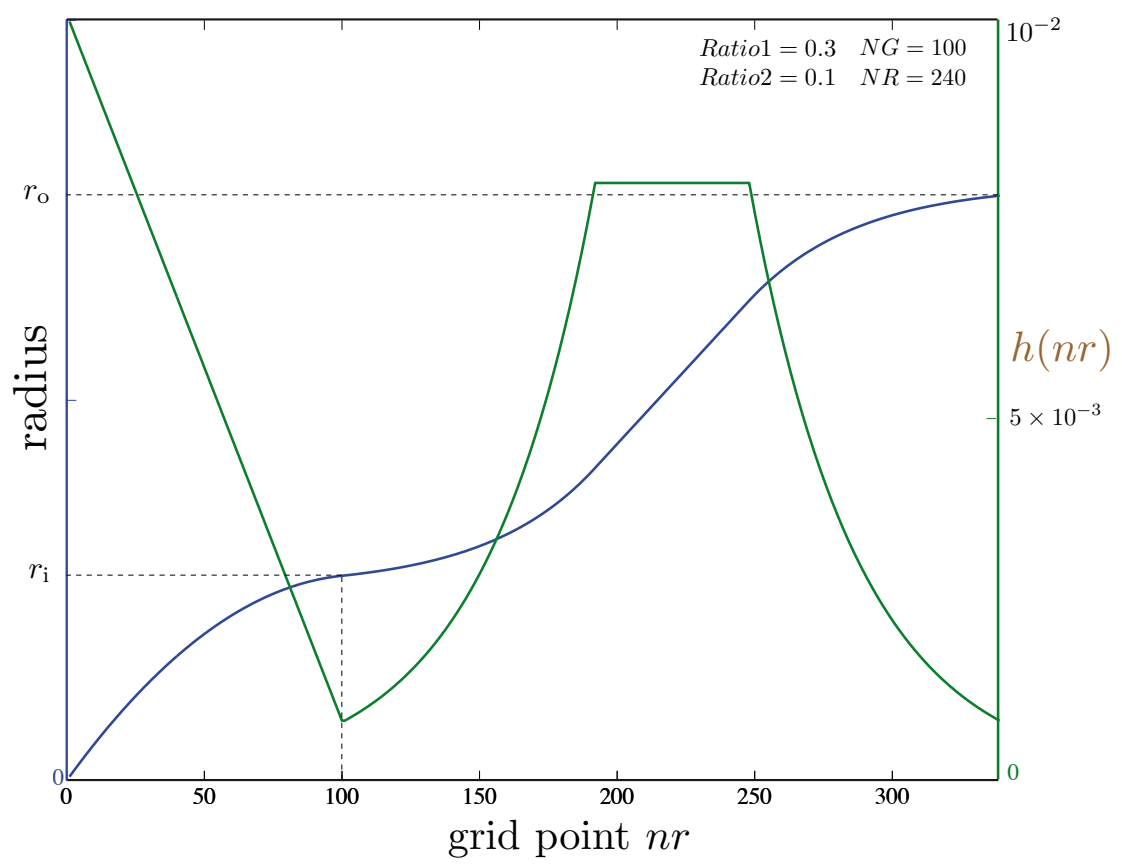

Figure 3.1: In blue, radius as a function of the grid point $n r$. In green, evolution of the interval $h$ between points. $N G=100$ are used in the inner core and $N R=240$ in the fluid outer core.

straightforward to determine the exact order of the radial scheme. However, this would correspond to schemes that degenerates like $h$ and $h^{2}$, for equations (3.10) and (3.11), in the case of a regular grid.

\subsubsection{Spherical harmonics expansion}

For a given vector $\mathbf{V}$, its poloidal and toroidal scalars, $V_{\mathrm{p}}$ and $V_{\mathrm{t}}$, are extended in spherical harmonics like

$$
\begin{aligned}
V_{\mathrm{t}}(r, \theta, \varphi) & =\sum_{\ell=0}^{\mathrm{L}_{\max }} \sum_{m=0}^{\ell} V_{\mathrm{t}_{\ell}}^{m}(r) Y_{\ell}^{m}(\theta, \varphi), \\
V_{\mathrm{p}}(r, \theta, \varphi) & =\sum_{\ell=0}^{\mathrm{L}_{\max }} \sum_{m=0}^{\ell} V_{\mathrm{p}_{\ell}}^{m}(r) Y_{\ell}^{m}(\theta, \varphi) .
\end{aligned}
$$

where $Y_{\ell}^{m}(\theta, \varphi)$ is the orthonormalized spherical harmonic function of degree $\ell$ and order $m$ defined as

$$
Y_{\ell}^{m}(\theta, \varphi)=P_{\ell}^{m}(\cos \theta) e^{i m \varphi},
$$

with $P_{\ell}^{m}$ the associated normalized Legendre polynomials. The transition between the spatial $(r, \theta, \varphi)$ to the spectral $(r, \ell, m)$ domain is performed by the SHTns library, developed by Schaeffer (2013). In such a representation, $\mathrm{L}_{\max }$ and $\mathrm{M}_{\max }$ are the maximum degree and 
order of the spherical harmonic expansion, corresponding to the lateral resolution of the system. From the construction of the spherical harmonic function, the Laplacian-Beltrami operator may be reduced to

$$
L_{2} V_{\mathrm{p} \ell}^{m}=\ell(\ell+1) V_{\mathrm{p}_{\ell}}^{m},
$$

which greatly simplifies the computation of equations (3.8) and (3.9) like

$$
\left(\frac{\partial}{\partial t}-\frac{\nabla^{2}}{P m}\right) B_{\mathrm{p}_{\ell}}^{m}=\frac{1}{\ell(\ell+1)} \mathbf{r} \cdot(\boldsymbol{\nabla} \times \mathbf{u} \times \mathbf{B}),
$$

and

$$
\left(\frac{\partial}{\partial t}-\frac{\nabla^{2}}{P m}\right) B_{\mathrm{t}_{\ell}}^{m}=\frac{1}{\ell(\ell+1)} \mathbf{r} \cdot(\boldsymbol{\nabla} \times(\boldsymbol{\nabla} \times \mathbf{u} \times \mathbf{B})) .
$$

Terms on the right-hand side of equations (3.16) and (3.17) are non linear terms, that are subject to specific treatment. They are calculated in the physical domain and then transferred into the spectral domain.

\subsubsection{Boundary conditions}

\section{Mechanical boundary conditions}

The stress-free boundary conditions expressed in section 2.2.7 of the previous chapter are transformed into conditions on the poloidal and toroidal scalars of the magnetic field, respectively $u_{\mathrm{p}}$ and $u_{\mathrm{t}}$. At the $\mathrm{ICB}\left(r=r_{\mathrm{i}}\right)$ and $\mathrm{CMB}\left(r=r_{\mathrm{o}}\right)$, this may be written

$$
\left\{\begin{aligned}
u_{\mathrm{p}} & =0 \\
\frac{\partial^{2} u_{\mathrm{p}}}{\partial r^{2}} & =0 \\
\frac{\partial}{\partial r}\left(\frac{u_{\mathrm{t}}}{r}\right) & =0
\end{aligned}\right.
$$

\section{Magnetic boundary conditions}

The magnetic boundary conditions are directly controlled by the interface conditions on the electrical $\mathbf{E}$ and magnetic $\mathbf{B}$ fields. Due to the existence of a thin conducting layer at the base of the mantle, the outer core boundaries are transitions between two electrically conducting media. In that case, the boundary conditions on the magnetic field involve the continuity of the toroidal scalar $B_{\mathrm{t}}$, as well as the continuity of the poloidal scalar $B_{\mathrm{p}}$ and its first radial derivative $\partial B_{\mathrm{p}} / \partial r$. However, such a transition generally involves a discontinuity in the first radial derivative of the toroidal field and a discontinuity on the second radial derivative of the poloidal field. As said previously, the inner core boundary is not a strict boundary for the magnetic field. The difference between ICB and CMB lies in the fact that, contrary to the inner core, the thin conducting layer at the base of the mantle is not physically implemented. This layer in then modeled by Neumann boundary 
conditions: conditions on the radial derivatives of $B_{\mathrm{p}}$ and $B_{\mathrm{t}}$ at the CMB. As boundary conditions between insulating and conducting media are similar for the poloidal scalar of the magnetic field, the condition that the magnetic field derives from a potential field leads to, in $r=r_{\mathrm{o}}+\Delta$,

$$
\frac{\partial B_{\mathrm{p}_{\ell}}^{m}}{\partial r}+\frac{\ell+1}{r} B_{\mathrm{p}_{\ell}}^{m}=0 .
$$

Contrary to the poloidal scalar, the radial derivative of the toroidal scalar is discontinuous at the interface between two conducting media. A condition on the latter may be obtained by considering the conservation of the tangential electric field at the very same interface (see e.g Holme (1998) and demonstration in Appendice A.1), and assuming the same magnetic permeability on both sides of the boundary. This leads to

$$
\left.r_{\mathrm{o}} \eta_{\mathrm{c}} \frac{\partial}{\partial r}\left(L_{2} B_{\mathrm{t}}\right)\right|_{\text {core }}+\mathbf{r} \cdot \nabla_{H} \times\left.(\mathbf{u} \times \mathbf{B})\right|_{\text {core }}=\left.r_{\mathrm{o}} \eta_{\mathrm{m}} \frac{\partial}{\partial r}\left(L_{2} B_{\mathrm{t}}\right)\right|_{\text {layer }},
$$

in which $\eta_{\mathrm{c}}$ and $\eta_{\mathrm{m}}$ are the magnetic diffusivity of the core and the mantle (conducting part) and $\boldsymbol{\nabla}_{H}$ the horizontal gradient, or even

$$
\left.\eta_{\mathrm{c}} \frac{\partial B_{\mathrm{t}_{\ell}}^{m}}{\partial r}\right|_{\text {core }}=\left.\eta_{\mathrm{m}} \frac{\partial B_{\mathrm{t}_{\ell}}^{m}}{\partial r}\right|_{\text {layer }}-\left.\frac{\left[\mathbf{r} \cdot \nabla_{H} \times(\mathbf{u} \times \mathbf{B})\right]_{\ell}^{m}}{r_{\mathrm{o}} \ell(\ell+1)}\right|_{\text {core }},
$$

when expanded on the spherical harmonic basis, where the Beltrami Laplacian operator of a given radial function $L_{2} h(r)$ is equal to $\ell(\ell+1) h(r)$.

The radial derivative on the core side is found to equal the radial derivative on the mantle side minus a non-linear advection term at the core-mantle boundary that is directly calculated. A condition on the radial derivative on the core side thus needs an assumption on the radial derivative in the mantle side. The main constraint on the mantle side is the continuity of the toroidal magnetic field at both CMB and between the conducting and insulating part of the mantle, at $r_{\mathrm{o}}+\Delta$. The first condition is then

$$
\left.B_{\mathrm{t}_{\ell}}^{m}\left(r_{\mathrm{o}}\right)\right|_{\text {core }}=\left.B_{\mathrm{t}_{\ell}}^{m}\left(r_{\mathrm{o}}\right)\right|_{\text {layer }} .
$$

However, as there are no current sources in the insulating part of the mantle, the continuity of the toroidal field in $r_{\mathrm{o}}+\Delta$ involves

$$
B_{\mathrm{t}_{\ell}}^{m}\left(r_{\mathrm{o}}+\Delta\right)=0
$$

In the implementation of Parody-JA code, the radial derivative in the mantle is chosen as to reach linearly the latter boundary condition (Eq. 3.23), so that

$$
\left.\frac{\partial B_{\mathrm{t}_{\ell}}^{m}}{\partial r}\right|_{\text {layer }}=-\frac{B_{\mathrm{t}_{\ell}}^{m}\left(r_{\mathrm{o}}\right)}{\Delta}
$$


The boundary condition on the radial derivative of the toroidal field is then, in $r=r_{\mathrm{o}}$,

$$
\left.\frac{\partial B_{\mathrm{t}_{\ell}}^{m}}{\partial r}\right|_{\text {core }}=-\frac{B_{\mathrm{t}_{\ell}}^{m}\left(r_{\mathrm{o}}\right)}{\Delta}-\left.\frac{\left[\mathbf{r} \cdot \nabla_{H} \times \mathbf{u} \times \mathbf{B}\right]_{\ell}^{m}}{\eta_{\mathrm{c}} r_{\mathrm{o}} \ell(\ell+1)}\right|_{\text {core }} .
$$

This implementation also considered that the electrical conductivity, and thus the magnetic diffusivity, of the layer at the base of the mantle is the same as in the core $\left(\eta_{\mathrm{c}}=\eta_{\mathrm{m}}\right)$. This, and the simplification of the radial derivative of $B_{t_{\ell}}^{m}$ on the mantle side, is supported by the thin layer approximation that is further developed in section 3.2.

\subsubsection{Time integration scheme}

The time integration involves a semi-implicit Crank-Nicolson scheme for the diffusion terms and an Adams-Bashforth for non linear terms. The first scheme is unconditionally stable while the second, as an explicit scheme, is conditionally stable, meaning that a particular attention is to be payed to the time step $d t$. Equations (3.16) and (3.17) may be viewed, in the generic form, as equations of the type

$$
\frac{\partial}{\partial t} \mathcal{A} f(t)=\mathcal{B} f(t)+g(t)
$$

where $\mathcal{A}$ and $\mathcal{B}$ can be laplacians or bi-laplacians, $f(t)$ the function to integrate and $g(t)$ representing the non linear terms. For a constant time-steping, the discretization gives

$$
\frac{\mathcal{A} f(t+d t)-\mathcal{A} f(t)}{d t} \approx \frac{1}{2} \mathcal{B} f(t)+\frac{1}{2} \mathcal{B} f(t+d t)+\frac{3}{2} g(t)-\frac{1}{2} g(t-d t),
$$

or even

$$
\left(\frac{\mathcal{A}}{d t}-\frac{1}{2} \mathcal{B}\right) f(t+d t) \approx\left(\frac{\mathcal{A}}{d t}+\frac{1}{2} \mathcal{B}\right) f(t)+\frac{3}{2} g(t)-\frac{1}{2} g(t-d t)
$$

\subsection{Parody-GP Code}

\section{Solution of the diffusion of magnetic field into the mantle}

We here establish the solution of a magnetic field diffusing into the electrically conducting part of the mantle, a thin layer of thickness $\Delta$ located on the mantle side of the CMB. In this layer, the diffusion of $\mathbf{B}$ is governed by

$$
\frac{\partial \mathbf{B}}{\partial t}+\nabla \times \eta_{\mathrm{m}}(\nabla \times \mathbf{B})=0
$$

with $\eta_{\mathrm{m}}$ the magnetic diffusivity of the layer. Introducing at characteristic scale of magnetic field $\mathcal{B}$ and a typical secular variation time scale $\tau_{S V}$ into equation (3.29) leads to the 
following the dimensional analysis,

$$
\frac{1}{\tau_{S V}} \mathcal{B}+\frac{\eta_{\mathrm{m}}}{\Delta^{2}} \mathcal{B}=0
$$

Here, $\Delta^{2} / \eta_{\mathrm{m}}$ represents the time $\tau_{\Delta}$ needed for a magnetic field to diffuse from the CMB $\left(r_{\mathrm{o}}\right)$ to $r_{\mathrm{o}}+\Delta$. This characteristic time can be estimated by considering that this layer should have a conductance $\left(\Delta \sigma_{\mathrm{m}}\right)$ on the order of $10^{8} \mathrm{~S}$, in order for the electromagnetic torque at the CMB to explain the variation of the length of day (LOD), with $\sigma_{\mathrm{m}}$ the electrically conductivity of the layer. This time may be expressed as

$$
\tau_{\Delta}=\mu_{0}\left(\Delta \sigma_{\mathrm{m}}\right) \Delta
$$

in which case its estimates only depends on the thickness $\Delta$ of the layer, that may be a thin layer having the conductivity of the core or a thicker layer of rocks having a smaller electrical conductivy, as identified in the introduction, section 1.6.2. For a thin conductive layer of $100 \mathrm{~m}$, we then find $\tau_{\Delta}=\mathrm{h}$, and for a thicker layer of $300 \mathrm{~km}$ we find that $\tau_{\Delta}=1.2$ yrs. In the following, we will thus rely the assumption that the characteristic time of the secular variation is much greater than the diffusion of the magnetic field through electrically conducting part of the mantle. This assumption can be written

$$
\tau_{S V} \gg \tau_{\Delta}
$$

We will thus neglect the time derivative of $\mathbf{B}$ in equation (3.29) and the magnetic field in the mantle is described by

$$
\boldsymbol{\nabla} \times \eta_{\mathrm{m}}(\boldsymbol{\nabla} \times \mathbf{B})=0 .
$$

Considering a uniform mantle conductivity $\left(\partial \eta_{\mathrm{m}} / \partial r=0\right)$ this can be summurized as

$$
\eta_{\mathrm{m}} \boldsymbol{\nabla} \times \nabla \times \mathbf{B}=0
$$

or even

$$
\boldsymbol{\nabla}(\boldsymbol{\nabla} \cdot \mathbf{B})-\nabla^{2} \mathbf{B}=0
$$

As $\mathbf{B}$ is a solenoidal field, this is equivalent to solve the Laplace equation in the electrically conducting part of the mantle.

$$
\nabla^{2} \mathbf{B}=0 .
$$

Using the poloidal-toroidal decomposition and the spherical harmonic expansion introduced in sections 3.1.2 and 3.1.3, we find that the toroidal field obeys

$$
\frac{\partial^{2}\left(r B_{\mathrm{t}_{\ell}}^{m}\right)}{\partial r^{2}}-\ell(\ell+1) \frac{\left(r B_{\mathrm{t}_{\ell}}^{m}\right)}{r^{2}}=0
$$


The final equation governing the diffusion of the toroidal magnetic field into the layer is obtain by noticing that the numerical implementation actually computes the quantity $r B_{\mathrm{t}_{\ell}}^{m}$. We will then solve the above equation considering that $B_{\mathrm{t}_{\ell}}^{m}=r B_{\mathrm{t}_{\ell}}^{m}$, a simplification that is convenient for the numerical implementation and facilitates the following developments. We finally obtain

$$
\frac{\partial^{2} B_{\mathrm{t}_{\ell}}^{m}}{\partial r^{2}}-\frac{\ell(\ell+1)}{r^{2}} B_{\mathrm{t}_{\ell}}^{m}=0
$$

\section{Thin layer approximation}

The thin layer approximation was firstly exposed by Stewart et al. (1995) and further studied by Holme (1998). It assumes that the magnetic field varies on horizontal scales that are large compared to the layer's thickness, and thus neglects the second term in equation (3.38). This equation is then be written like

$$
\frac{\partial^{2} B_{\mathrm{t}_{\ell}}^{m}}{\partial r^{2}}=0
$$

Considering that the toroidal field must be zero in $r_{\mathrm{o}}+\Delta$ leads to the following solution, $\forall r \in\left[r_{\mathrm{o}} ; r_{\mathrm{o}}+\Delta\right]$

$$
B_{\mathrm{t}_{\ell}}^{m}(r)=B_{\mathrm{t}_{\ell}}^{m}\left(r_{\mathrm{o}}\right) \frac{r_{\mathrm{o}}+\Delta-r}{\Delta},
$$

where $B_{\mathrm{t}_{\ell}}^{m}\left(r_{\mathrm{o}}\right)$ is the value of the toroidal scalar of the magnetic field at the core-mantle boundary. This leads to the Neumann boundary condition implemented in the Parody-JA code as expressed in section 3.1.4.

\section{Implementation in Parody-GP}

The Parody-GP code includes a treatment of the solution to equation (3.38) that gets rid of the thin layer approximation. The general solution from $B_{\mathrm{t}_{\ell}}^{m} \sim r^{\alpha}$ is found to be of the form

$$
B_{\mathrm{t}_{\ell}}^{m}(r)=C_{1} r^{-\ell}+C_{2} r^{\ell+1}
$$

with $C_{1}$ and $C_{2}$ two constants to be determined. The solution is constrained by the same boundary conditions: the value of the toroidal scalar at the CMB is $B_{\mathrm{t}_{\ell}}^{m}\left(r_{\mathrm{o}}\right)$ and is equal zero in $r_{\mathrm{o}}+\Delta, B_{\mathrm{t}_{\ell}}^{m}\left(r_{\mathrm{o}}+\Delta\right)=0$. In $r_{\mathrm{o}}+\Delta$ we have,

$$
0=C_{1}\left(r_{\mathrm{o}}+\Delta\right)^{-\ell}+C_{2}\left(r_{\mathrm{o}}+\Delta\right)^{\ell+1}
$$

or even

$$
C_{1}=-C_{2}\left(r_{\mathrm{o}}+\Delta\right)^{2 \ell+1}
$$


so that

$$
\begin{aligned}
B_{\mathrm{t}_{\ell}}^{m} & =-C_{2}\left(r_{\mathrm{o}}+\Delta\right)^{2 \ell+1} r^{-\ell}+C_{2} r^{\ell+1}, \\
& =C_{2}\left(r^{\ell+1}-\frac{\left(r_{\mathrm{o}}+\Delta\right)^{2 \ell+1}}{r^{\ell}}\right) .
\end{aligned}
$$

At the CMB, in $r=r_{\mathrm{o}}$, we have

$$
B_{\mathrm{t}_{\ell}}^{m}\left(r_{\mathrm{o}}\right)=C_{2}\left(r_{\mathrm{o}}^{\ell+1}-\frac{\left(r_{\mathrm{o}}+\Delta\right)^{2 \ell+1}}{r_{\mathrm{o}}^{\ell}}\right),
$$

leading to

$$
C_{2}=\frac{B_{\mathrm{t}_{\ell}}^{m}\left(r_{\mathrm{o}}\right)}{\left(r_{\mathrm{o}}^{\ell+1}-\frac{\left(r_{\mathrm{o}}+\Delta\right)^{2 \ell+1}}{r_{\mathrm{o}}^{\ell}}\right)} .
$$

Introducing the expressions of $C_{1}$ and $C_{2}$ into solution (3.41) then gives

$$
B_{\mathrm{t}_{\ell}}^{m}(r)=\frac{B_{\mathrm{t}_{\ell}}^{m}\left(r_{\mathrm{o}}\right)}{\left(r_{\mathrm{o}}^{\ell+1}-\frac{\left(r_{\mathrm{o}}+\Delta\right)^{2 \ell+1}}{r_{\mathrm{o}}^{\ell}}\right)}\left(r^{\ell+1}-\frac{\left(r_{\mathrm{o}}+\Delta\right)^{2 \ell+1}}{r^{\ell}}\right),
$$

and finally

$$
B_{\mathrm{t}_{\ell}}^{m}(r)=B_{\mathrm{t}_{\ell}}^{m}\left(r_{\mathrm{o}}\right)\left(\frac{r_{\mathrm{o}}}{r}\right)^{\ell}\left[\frac{r^{2 \ell+1}-\left(r_{\mathrm{o}}+\Delta\right)^{2 \ell+1}}{r_{\mathrm{o}}^{2 \ell+1}-\left(r_{\mathrm{o}}+\Delta\right)^{2 \ell+1}}\right] .
$$

From equation (3.49) we can now express the radial derivative of the toroidal scalar for the magnetic field at the mantle side of the CMB,

$$
\left.\frac{\partial B_{\mathrm{t}_{\ell}}^{m}}{\partial r}\right|_{\text {layer }}=\frac{B_{\mathrm{t}_{\ell}}^{m}\left(r_{\mathrm{o}}\right)}{r_{\mathrm{o}}}\left[\frac{(\ell+1) r_{\mathrm{o}}^{2 \ell+1}-\ell\left(r_{\mathrm{o}}+\Delta\right)^{2 \ell+1}}{r_{\mathrm{o}}^{2 \ell+1}-\left(r_{\mathrm{o}}+\Delta\right)^{2 \ell+1}}\right],
$$

that is to be integrated in the boundary condition on the toroidal field, in order to obtain the expression of the radial derivative of the latter in the core side of the CMB. We then obtain

$$
\left.\frac{\partial B_{\mathrm{t}_{\ell}}^{m}}{\partial r}\right|_{\text {core }}=\frac{\eta_{\mathrm{m}}}{\eta_{\mathrm{c}}} \frac{B_{\mathrm{t}_{\ell}}^{m}\left(r_{\mathrm{o}}\right)}{r_{\mathrm{o}}} \mathcal{F}(\ell)-\left.\frac{\mathbf{r} \cdot \boldsymbol{\nabla}_{H} \times \mathbf{u} \times \mathbf{B}}{\eta_{\mathrm{c}} r_{\mathrm{o}} \ell(\ell+1)}\right|_{\text {core }},
$$

with

$$
\mathcal{F}(\ell)=\left[\frac{(\ell+1) r_{\mathrm{o}}^{2 \ell+1}-\ell\left(r_{\mathrm{o}}+\Delta\right)^{2 \ell+1}}{r_{\mathrm{o}}^{2 \ell+1}-\left(r_{\mathrm{o}}+\Delta\right)^{2 \ell+1}}\right] .
$$

The function $\mathcal{F}(\ell)$ is here expressed as having for only parameter the degree $\ell$ of the spherical harmonic expansion since, for a given numerical simulation, the thickness of the conducting layer is an input parameter. It though depends on $\Delta$ as expressed by its Taylor's expansion 
in the vicinity of small $\Delta$,

$$
\mathcal{F}(\ell)=\frac{r_{\mathrm{o}}}{\Delta}-\ell, \quad \text { for } \quad \Delta / r_{\mathrm{o}} \mapsto 0
$$

A comparison between the thin layer approximation solution $\left(-r_{\mathrm{o}} / \Delta\right)$, the full solution given by $\mathcal{F}(\ell)$ and its Taylor's expansion is presented in figure 3.2. This figure shows that

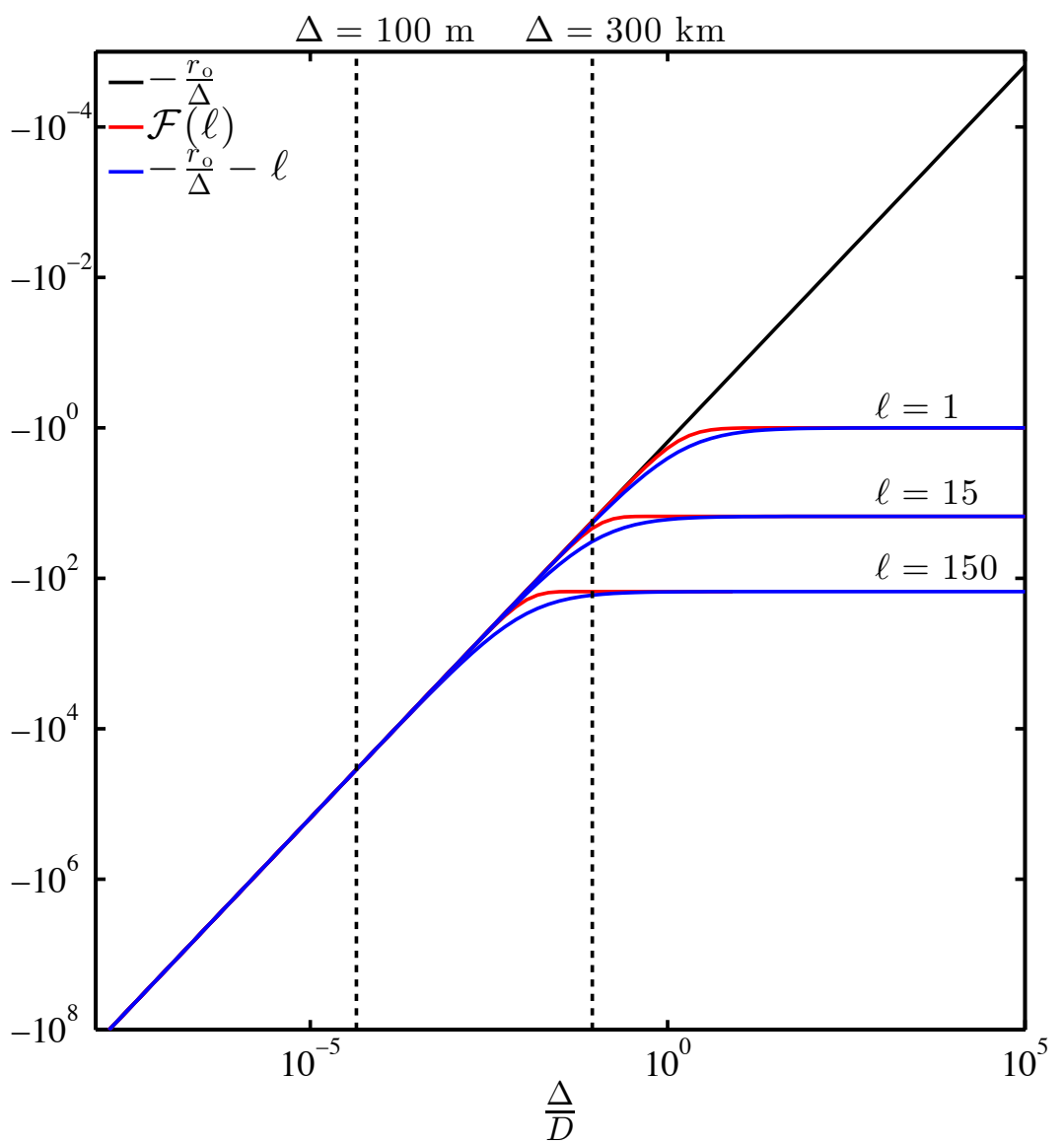

Figure 3.2: In black, the thin layer approximation solution $\left(-r_{\mathrm{o}} / \Delta\right)$ as implemented in JA-Parody. The complete solution (Eq. 3.52) and its Taylor's expansion (Eq. 3.53) are respectively in red and black. Values of these solutions are given as a function of the dimensionless thickness of the conducting layer $(\Delta / D)$, and different degrees of the $\mathrm{SH}$ expansion. Black doted lines represent two extreme scenarios of layer's thickness.

the thin layer solution is not far from the complete solution for layer thickness smaller than $300 \mathrm{~km}$. This thus demonstrates that this approximation may be sufficient to model the diffusion of the magnetic field from the core into such a layer. The solution is however affected by the increase of the spherical harmonic $\ell$, in the range of possible layer thickness.

The Parody-GP code integrates the complete solution developed in this section. Moreover, in order to account for different scenarios of electrically conducting layers at the base of the mantle, the original conductance input parameter is now splitted into two input parameters: the thickness of the conducting layer $\Delta$ and its electrical conductivity $\sigma_{\mathrm{m}}$. 


\subsubsection{Torque components}

\section{Poloidal torque}

The electromagnetic torque acting on the mantle is often decomposed in poloidal and toroidal components Rochester (1960, 1962); Stix and Roberts (1984); Holme (1998), that are respectively generated by poloidal and toroidal induced currents in the mantle. For a given electromagnetic torque $\Gamma_{\mathrm{EM}}$, this decomposition may be written

$$
\Gamma_{\mathrm{CMB}}=\Gamma_{\mathrm{P}}+\Gamma_{\mathrm{T}}
$$

with $\Gamma_{\mathrm{P}}$ and $\Gamma_{\mathrm{P}}$ the poloidal and toroidal parts of the torque. Expressions of $\Gamma_{\mathrm{P}}$ and $\Gamma_{\mathrm{T}}$ may be obtained by considering the volume integral of the Lorentz force induced by the penetration of poloidal and toroidal induced currents (see e.g Stix and Roberts, 1984; Holme, 1998),

$$
\Gamma_{\mathrm{P}}=-\frac{1}{\mu_{0}} \int_{S} \frac{\partial\left(\nabla^{2} B_{\mathrm{p}}\right)}{\partial \varphi} B_{\mathrm{r}} r \mathrm{dS},
$$

with $B_{\mathrm{s}}$ the solenoidal part of the magnetic field which may be related to the poloidal part $B_{\mathrm{p}}$ like

$$
B_{\mathrm{s}}=\frac{1}{r} \frac{\partial}{\partial r}\left(r B_{\mathrm{p}}\right)
$$

At the CMB, however, the boundary condition on the poloidal field is chosen as to match a potential field, which has no associated currents. In such a situation, we necessarily have $\nabla^{2} B_{\mathrm{p}}=0$, and consequently $\Gamma_{\mathrm{P}}=0$. Our implementation thus only computes the toroidal part of the electromagnetic torque acting on the mantle, which is often considered as several orders of magnitude greater than the poloidal part.

\section{Toroidal torque}

Reconstructions of LOD variations induced by core-mantle electromagnetic coupling are often based on the toroidal part of the torque $\Gamma_{\mathrm{T}}$. The expression of $\Gamma_{\mathrm{T}}$ may be obtained by introducing the toroidal part of azimuthal the magnetic field into the surface integral of the total torque. Using the decomposition given by system (3.2), we then find that

$$
\Gamma_{\mathrm{T}}=\frac{r_{\mathrm{o}}}{\mu_{0}} \int_{S_{C M B}} \frac{\partial B_{\mathrm{t}}}{\partial \theta} B_{\mathrm{r}} \sin \theta \mathrm{dS}
$$

where $B_{\mathrm{t}}$ and $B_{\mathrm{r}}$ are the values of the field at the core mantle boundary. As further developed in section 3.2, the value of the toroidal magnetic field at the CMB (and its radial derivative) is the combination of diffusion of toroidal field from the core and advection at the CMB. Thus, the toroidal torque may be decomposed into the classically defined "leakage" and "advective" torques. The advective torque acting on the mantle may be computed by core-flow inversions, the value of the toroidal field at the CMB is then deduced from the computation of advection term, that is proportional to the strength of the radial magnetic 
field and the inferred core flow. However, since the toroidal field does not penetrate the insulating mantle, it is not observable at Earth's surface. This method thus ignores the toroidal field that diffuses from the core to the CMB, and so neglects the "leakage" torque. Though it seems unlikely that the toroidal field from the core suffer decadal oscillations, this torque is generally considered as irrelevant to explain the decadal LOD variations (Jault and Le Mouël, 1991).

\section{Implementation}

Given the demonstration on boundary condition on the toroidal magnetic field developed in section 3.2, we found that it would be of interest to have insights on the "leakage" torque from our numerical simulations of the geodynamo. We thus decompose and retrieve the advective and diffusive parts of the toroidal field at the core-mantle boundary, and computes two different integrals that express the advective and the leakage torque on the mantle, respectively $\Gamma_{\mathrm{AD}}$ and $\Gamma_{\text {LEAK }}$. Figure 3.3 exposes the results of both the advective and leakage torque, as proportions of the time-averaged total torque $\left\langle\Gamma_{\mathrm{CMB}}\right\rangle$. In this typical simulation, the leakage torque is found to represent only a few percents of the total torque, with smaller variations around the mean $\left(8 \pm 3 \%\right.$ of $\left\langle\Gamma_{\mathrm{CMB}}\right\rangle$ in this case).

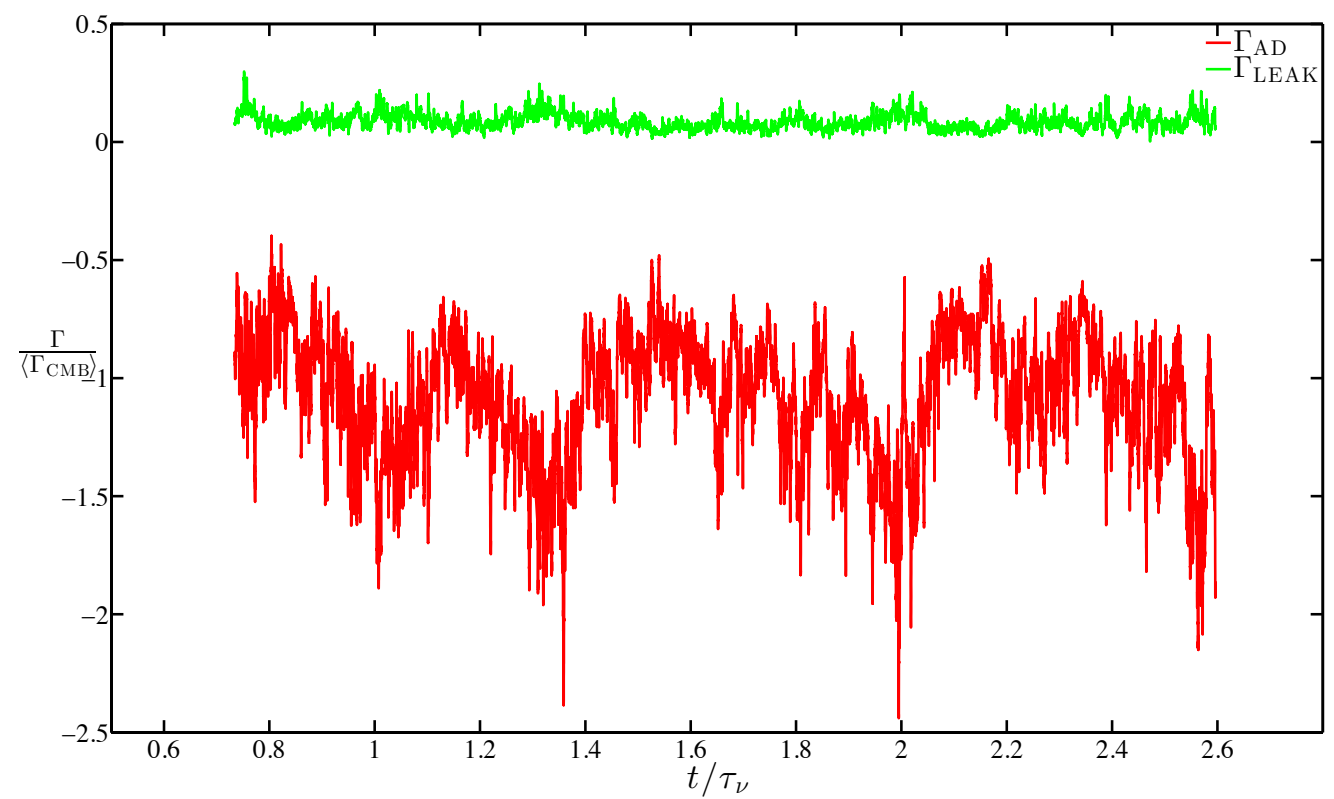

Figure 3.3: Leakage $\left(\Gamma_{\mathrm{LEAK}}\right)$ and advective torque $\left(\Gamma_{\mathrm{AD}}\right)$ divided by the mean total torque at the core-mantle boundary $\left\langle\Gamma_{\mathrm{CMB}}\right\rangle$ as a function of the dimensionless viscous time.

\subsubsection{Time-dependent gravitational torque}

In our system, both mantle and inner core experience a gravitational torque $\Gamma_{G}$, that is proportional to mantle heterogeneities of density and inner core's viscosity. Mantle heterogeneities are embodied by a gravitational coupling constant $\Gamma$, which corresponds to 
the strength of the gravitational torque, while the inner core viscosity $\mu_{\mathrm{ic}}$ is represented by a viscous relaxation time scale

$$
\tau \propto \frac{\mu_{\mathrm{ic}}}{\Delta \rho g_{\mathrm{ic}} r_{\mathrm{i}}},
$$

in the case of a uniform inner core (Buffett, 1997), with $\Delta \rho$ the density jump and $g_{\text {ic }}$ the acceleration of gravity at the ICB. The gravitational torque exerted on the inner core is given by

$$
\Gamma_{\mathrm{G}}=-\phi \Gamma
$$

where $\phi$ is the misalignment angle between mantle heterogeneities and inner core deformations. The evolution of $\phi$ over time thus is proportional to the angular velocities of the inner core $\Omega_{\mathrm{ic}}$ and the mantle $\Omega_{\mathrm{m}}$ like

$$
\frac{\mathrm{d} \phi}{\mathrm{dt}}=\Omega_{\mathrm{ic}}-\Omega_{\mathrm{m}}-\frac{\phi}{\tau} .
$$

\section{Long-term}

The long-term implementation of the gravitational torque is based on the assumption that

$$
\frac{\mathrm{d} \phi}{\mathrm{dt}} \ll \frac{\phi}{\tau} .
$$

This is strictly valid when only considering a steady-state of a constant inner core superrotation, and should still be relevant for decadal oscillations of the inner core differential rotation inferred by Tkalčić et al. (2013) if the relaxation time scale of the inner core does not exceed the decade. In the framework of this assumption, the misalignment angle becomes

$$
\phi=\left(\Omega_{\mathrm{ic}}-\Omega_{\mathrm{m}}\right) \tau
$$

and the expression of the gravitational torque exerted by the mantle on the inner core reduces to

$$
\Gamma_{\mathrm{G}}=-\left(\Omega_{\mathrm{ic}}-\Omega_{\mathrm{m}}\right) \tau \Gamma \text {. }
$$

If the inner core is subject to both gravitational and electromagnetic torques, the conservation of the inner core's angular momentum, with a constant moment of inertia $I_{i}$ is then

$$
\begin{aligned}
\mathrm{I}_{\mathrm{i}} \frac{\mathrm{d} \Omega_{\mathrm{ic}}}{\mathrm{dt}} & =\Gamma_{\mathrm{ICB}}+\Gamma_{\mathrm{G}}, \\
& =\left(\Gamma_{\mathrm{ICB}}-\left(\Omega_{\mathrm{ic}}-\Omega_{\mathrm{m}}\right) \tau \Gamma\right) .
\end{aligned}
$$

In order to fit the discretization process we write

$$
\frac{\mathrm{d} \Omega_{\mathrm{ic}}}{\mathrm{dt}}=\frac{\Omega_{\mathrm{ic}} \tau \Gamma}{\mathrm{I}_{\mathrm{i}}}+\frac{1}{\mathrm{I}_{\mathrm{i}}}\left(\Gamma_{\mathrm{ICB}}-\left(-\Omega_{\mathrm{m}}\right) \tau \Gamma\right) .
$$


Taking $g(t)=\Gamma_{\mathrm{ICB}}(t)+\tau \Gamma \Omega_{\mathrm{m}}(t)$, and using the Adams-Bashforth time scheme introduced in section 3.1.2, we then have

$$
\left(\frac{1}{d t}+\frac{\tau \Gamma}{2 \mathrm{I}_{\mathrm{i}}}\right) \Omega_{\mathrm{ic}}(t+d t)=\left(\frac{1}{d t}-\frac{\tau \Gamma}{2 \mathrm{I}_{\mathrm{i}}}\right) \Omega_{\mathrm{ic}}(t)+\frac{1}{\mathrm{I}_{\mathrm{i}}}\left(\frac{3}{2} g(t)-\frac{1}{2} g(t-d t)\right)
$$

and finally,

$$
\Omega_{\mathrm{ic}}(t+d t)=\left(\frac{1-d t \frac{\tau \Gamma}{2 \mathrm{I}_{\mathrm{i}}}}{1+d t \frac{\tau \Gamma}{2 \mathrm{I}_{\mathrm{i}}}}\right) \Omega_{\mathrm{ic}}(t)+\frac{d t}{\mathrm{I}_{\mathrm{i}}}\left(\frac{3}{2} g(t)-\frac{1}{2} g(t-d t)\right)
$$

The value of the inner core rotation at step $t+d t$ is thus controlled by the strength of gravitational coupling, the mantle rotation and the electromagnetic torque at time $t$ and $t+d t$. In this implementation, the magnitude of the gravitational coupling is embodied by a single parameter $\tau \Gamma$.

\section{Time-dependent}

If we no longer rely on the long-term expression of gravitational torque, we have to solve a set of equations

$$
\left\{\begin{aligned}
\frac{\mathrm{d} \Omega_{\mathrm{ic}}}{\mathrm{dt}} & =-\frac{\phi \Gamma}{\mathrm{I}_{\mathrm{i}}}+\frac{\Gamma_{\mathrm{ICB}}}{\mathrm{I}_{\mathrm{i}}} \\
\frac{\mathrm{d} \phi}{\mathrm{dt}} & =\mathcal{S}-\frac{\phi}{\tau}
\end{aligned}\right.
$$

with $\mathcal{S}=\Omega_{\mathrm{ic}}-\Omega_{\mathrm{m}}$, the inner core super-rotation. Using $g_{\phi}(t)=\phi(t) \Gamma+\Gamma_{\mathrm{ICB}}(t)$, discretization of the first equation of system (3.69) is then

$$
\Omega_{\mathrm{ic}}(t+d t)=\Omega_{\mathrm{ic}}(t)+\frac{d t}{\mathrm{I}_{\mathrm{i}}}\left(\frac{3}{2} g_{\phi}(t)-\frac{1}{2} g_{\phi}(t-d t)\right)
$$

while the equation describing the misalignment angle becomes

$$
\phi(t+d t)=\left(\frac{1-\frac{d t}{2 \tau}}{1+\frac{d t}{2 \tau}}\right) \phi(t)+d t \times\left(\frac{3}{2} \mathcal{S}(t)-\frac{1}{2} \mathcal{S}(t-d t)\right)
$$

The exact description of the gravitational torque acting on the core, that leads to equations (3.70) and (3.71) in an explicit scheme, is implemented in the Parody-GP code. This provides the advantages of a better characterization of fluctuations in the inner core differential rotation with periodicities shorter than the decade. Moreover, this formalism offers a decoupling of the two main parameters of the gravitational torque, $\tau$ and $\Gamma$, which are now proposed as input parameters. It thus allows the study of a new parameter space, with an inner core having a small viscosity that is subject to weak or strong mantle heterogeneities anomalies, leading to small or high $\Gamma$ values, for example. 


\section{Long-term rotational dynamics}

\section{Résumé}

Ce chapitre est une reproduction de l'article de recherche "Coupled dynamics of Earth's geomagnetic westward drift and inner core super-rotation" (G.Pichon, J.Aubert, A.Fournier) publié dans Earth and Planetary Science Letters. Il présente une étude systématique du comportement au long terme de la dérive géomagnétique vers l'ouest et de la super-rotation de la graine, en tant que composants de la dynamique rotationnelle du noyau terrestre, à partir de simulations numériques de la géodynamo. Ces simulations intègrent un couplage gravitationnel entre le manteau et le noyau interne, ainsi que deux couples électromagnétiques aux frontières du noyau fluide. Cette étude montre que l'amplitude de ces couples n'influence pas le cisaillement global disponible dans le noyau fluide, dont la valeur est entièrement déterminée par la vigueur de la convection. L'amplitude relative de ces couples gouverne cependant la distribution de ce cisaillement entre la dérive géomagnétique vers l'ouest et la super-rotation de la graine. La valeur estimée de ce cisaillement est remarquablement proche de celle de la dérive géomagnétique vers l'ouest moyenne durant les 400 dernières années, ce qui suggère que l'intégralité de ce cisaillement est consommée par cette dernière. De ce fait, la super-rotation de la graine est nécessairement très proche, ou égale, à zéro en moyenne. En supposant que la conductance du manteau est de l'ordre de $10^{8} \mathrm{~S}$, cette conclusion permet alors de contraindre la viscosité de la graine comme étant supérieure à $2 \times 10^{17}$ Pa.s. 


\title{
Coupled dynamics of Earth's geomagnetic westward drift and inner core super-rotation
}

\author{
Guillaume Pichon*, Julien Aubert, Alexandre Fournier \\ Institut de Physique du Globe de Paris, Sorbonne Paris Cité, Université Paris-Diderot, UMR 7154 CNRS, 1 rue Jussieu, F-75005 Paris, France
}

\begin{abstract}
The geomagnetic westward drift and the inner core differential rotation are two components of the Earth's core rotational dynamics. We present a systematic study of their long-term relationship in convective numerical simulations of the geodynamo. All models comprise gravitational coupling between the inner core and the mantle, in addition to electromagnetic coupling at the inner core and core-mantle boundaries. We show that the strength of these couplings has no influence on the global shear available in the fluid shell, the amount of which is entirely governed by the vigor of convection. This shear is distributed between the long-term westward drift and the long-term differential rotation of the inner core, in proportions controlled by the relative magnitudes of the electromagnetic and gravitational couplings. A present-day estimate of this available shear predicts a magnitude of the westward drift close to that observed on average during the last 400 years, which then implies a non-existent long-term inner core differential rotation. Assuming a lower mantle conductance of order $10^{8} \mathrm{~S}$, this in turn sets a constraint on the minimum stiffness of the inner core, the viscosity of which should be larger than $2 \times 10^{17} \mathrm{~Pa}$.s for the westward drift to dominate.
\end{abstract}

\subsection{Introduction}

The geomagnetic westward drift and the inner core super-rotation are two apparently distinct signatures of rotational dynamics in the Earth's core. The understanding of their behavior is of interest to several disciplines: geomagnetism, seismology, geodynamics and geodesy. However, their possible relationship has so far received little attention.

One of the striking results of the pioneering geodynamo simulation of Glatzmaier and Roberts (1996) was the observation of a differential rotation of the inner core respectively to the mantle of about $2^{\circ}$ per year. This was obtained by taking into account the electromagnetic coupling between the electrically conducting fluid outer core and solid inner core. Since the inner core is electrically conducting, it can indeed be permeated by a toroidal magnetic field which creates an axial electromagnetic torque (Gubbins, 1981). This generally promotes co-rotation between the inner core and the overlying fluid in the outer core, but local induction effects at the inner core boundary (ICB) allow for a residual angular velocity 
jump (Aurnou et al., 1996), with the angular velocity difference at the ICB opposing the shear in the outer core. The situation is hence similar to that occurring in an asynchronous motor.

Initial numerical reports of the inner core differential rotation (Glatzmaier and Roberts, 1996) gave rise to numerous seismological studies. Using differential travel-time between PKP(BC) and PKP(DF) waves, Song and Richards (1996) inferred a rotation rate of the inner core of $1.1 \pm 0.7^{\circ} \mathrm{yr}^{-1}$ in the eastward direction. Since then, many authors have revised downwards the initial estimates to a maximum rotation rate of about $0.3^{\circ} \mathrm{yr}^{-1}$, for instance by using temporal variations in seismic waves travel-time (Creager, 1997; Song, 2000). Using normal modes, Laske and Masters (1999) concluded there was an absence of inner core super-rotation, and this is the accepted scenario at the present time (see Souriau and Calvet, 2015, for a review). Therefore, the seismologically inferred super-rotation may correspond to decadal fluctuations (Tkalčić et al., 2013) around an average zero differential rotation, as initially suggested by Song and Poupinet (2007).

The geomagnetic westward drift is, in contrast, much better constrained. It was first described by Halley (1692), as a westward drift of agonic lines of the Earth's magnetic field over time. It is nowadays well imaged over the last four centuries in models accounting for data acquired by mariners, observatories and satellites, as the westward drift of magnetic flux patches at the core surface (Jackson et al., 2000; Finlay and Jackson, 2003). Concentrated at the equator in the Atlantic hemisphere, these patches have an average longitudinal velocity of 17 kilometers per year over the epochs investigated by Finlay and Jackson (2003), which corresponds to an angular velocity of $0.28^{\circ} \mathrm{yr}^{-1}$.

Since Roberts $\mathrm{P}$ and Scott (1965), it is admitted that this secular variation pattern reflects advection by an underlying azimuthal flow below the core-mantle boundary (CMB). At this point, it is thus natural to imagine that the westward drift and the inner core super-rotation are the top and bottom signatures of a global radial shear in azimuthal velocities. The dynamic origin of such a global shear may reside in core polar vortices. These vortices result from the interaction between convective upwellings and Coriolis forces inside the tangent cylinder (the imaginary cylinder aligned with the rotation axis and circumscribing the inner core). They are cyclonic close to the ICB and anti-cyclonic when approaching the CMB. These polar vortices are ubiquitous features in numerical geodynamo simulations (e.g. Aubert, 2005; Sreenivasan and Jones, 2006), and appear to be in agreement with flows estimated from the geomagnetic secular variation (Olson and Aurnou, 1999), though they should be interpreted with caution as they are poorly resolved in polar regions (Eymin and Hulot, 2005). Polar vortices can provide the shear that links the inner core rotation and the westward drift, but the absolute velocities of these quantities in the frame rotating with the Earth are ultimately determined by the state of coupling between the inner core, the outer core and the mantle (Dumberry, 2007; Aubert et al., 2013).

The key ingredient here is the possibility of a gravitational torque, coupling the inner core and the mantle (Buffett, 1996b). The mechanism involves density anomalies in the 
mantle (see Davies et al. (2014) for recent estimates) which deform the equipotential gravity surfaces by about a hundred meters close to the ICB (Buffett, 1997). The shape of the inner core adjusts to the gravity equipotential, in which case a slight misalignment between the mantle and the inner core results in a strong restoring force. If the inner core is allowed to viscously deform, though, then a super-rotation is still worth considering while creating a moderate restoring force (Buffett, 1997). A second crucial component is the coupling between the outer core and the mantle at the CMB.Moreover, the strength of the gravitational torque also affects the average westward flow at the CMB (Buffett and Glatzmaier, 2000), as a consequence of the balance between this remote torque and a direct torque between the mantle and the fluid at the CMB. A primary candidate for a such coupling is again electromagnetic forces (Buffett, 1992; Holme, 1998; Buffett and Christensen, 2007). The basic idea is that there is an electrically conducting layer on the mantle side of the CMB, which again can be permeated by toroidal magnetic fields and hence can experience a magnetic torque (Rochester, 1960, 1962). Evidence for the existence of this layer can be obtained by looking at the out-of-phase component of the forced nutations of the Earth (Buffett, 1992; Buffett et al., 2002), which constrains the conductance of this layer to be at least $10^{8} \mathrm{~S}$. This is supported by recent mineral physics experiments, inferring a thick post-perovskite layer (Murakami et al., 2004) of quite high conductivity (Ohta et al., 2008) or a thin layer of metallic FeO with a conductivity close to that of the core (Ohta et al., 2012).

From this discussion, it becomes obvious that the rotational state of the core is the result of a complex combination of physical effects. For example, the angular velocity jump at the ICB will be influenced by the strength of the gravitational torque exerted on the inner core and the amount of westward drift will crucially be determined by the amplitude of magnetic coupling at the CMB. We can however anticipate that the global amount of shear available in the outer core will be insensitive to both effects described above, as it should only be dictated by the strength of convection (Aubert, 2005). It thus appears timely to elaborate numerical geodynamo models encompassing all these effects and derive the scaling laws governing the long-term rotation components of Earth's coupled core-mantle system. From a practical standpoint, this is an incremental study adding the CMB magnetic coupling to the coupled Earth dynamo system which neglected its impact (Aubert et al., 2013). From a theoretical point of view, we rely on the theory developed by Dumberry (2007) in order to derive the scaling laws. We frame our analysis within the thermal wind theory (Aurnou et al., 2003; Aubert, 2005) to explain the geomagnetic westward drift. This theory is able to reproduce several observations of the Earth's magnetic field and its secular variation, such as the equatorial field patch trains of normal polarity, their wave like patterns and their inferred underlying core flows, all within a self-consistent convective model (see e.g. Aubert et al., 2013). There are alternative theories to explain the geomagnetic westward drift : magnetic winds (Livermore et al., 2013), magnetic Rossby waves (Hori et al., 2015) and mantle control (Christensen and Olson, 2003); however, these have yet to give birth to dynamical models 
capable of generating spontaneously the salient features of the geomagnetic secular variation we just recalled. We also restrict our analysis to electromagnetic and gravitational torques only, and do not consider other sources of coupling between the mantle and the core, such as the topographic torque, which remains poorly constrained (Roberts and Aurnou, 2012). The key geophysical questions we have in mind are the following ones: are the inner core super-rotation and the westward drift long-term features of the geodynamo? What is the physical link between these two components of the rotational dynamics of the Earth? Under what conditions does the coupled Earth dynamo model match the observed westward drift? To address these questions, we dedicate the second section to the description of our physical model, its numerical implementation, and a theoretical analysis of its long-term rotational state. That theory is successfully tested against the outputs of numerical simulations in section 4.3, and its geophysical implications are finally discussed in section 4.4.

\subsection{Model}

\subsubsection{Conservation laws}

We consider the flow of an electrically conducting, incompressible fluid of density $\rho$ and viscosity $\nu$, driven by convection in a spherical shell of thickness $D=r_{\mathrm{o}}-r_{\mathrm{i}}$, where $r_{\mathrm{i}}$ is the inner core radius and $r_{\mathrm{o}}$ is the core-mantle boundary radius. A set of spherical coordinates is chosen as $(r, \theta, \varphi)$, with associate unit vectors $\left(\mathbf{e}_{\mathrm{r}}, \mathbf{e}_{\theta}, \mathbf{e}_{\varphi}\right)$. The shell is rotating at an angular velocity $\Omega$ about an axis $\mathbf{e}_{\mathrm{z}}$, and its aspect ratio $r_{\mathrm{i}} / r_{\mathrm{o}}=0.35$ is that of the present-day Earth's core. As the fluid is assumed to be incompressible, the continuity equation describes the velocity field $\mathbf{u}$ as solenoidal,

$$
\nabla \cdot \mathbf{u}=0
$$

Thermochemical convection is modeled by the codensity $C$ (Braginsky and Roberts, 1995) in the Boussinesq approximation, such that

$$
C=\alpha_{T} \rho T^{\prime}+\Delta \rho \xi^{\prime}
$$

with $\alpha_{T}$ the thermal expansion coefficient, $T^{\prime}$ the deviation of the temperature field about the isentropic temperature, $\Delta \rho$ the density difference between light elements and pure iron and $\xi^{\prime}$ the light element mass fraction with respect to a well-mixed outer core. We assume that thermal and chemical diffusity are both equal to $\kappa$, the codensity diffusivity, due to turbulent mixing in the outer core. Thus, the codensity field $C$ is given by a single transport equation,

$$
\frac{\partial C}{\partial t}+\mathbf{u} \cdot \nabla C=\kappa \nabla^{2} C+S_{T / \xi}
$$

where $S_{T / \xi}$ is a volumetric correction term ensuring mass conservation (Aubert et al., 
2009). To obtain the velocity $\mathbf{u}$, codensity $C$ and magnetic fields $\mathbf{B}$ we solve equations (4.1) and (4.3) together with the electromagnetic induction equation in the magnetohydrodynamic approximation and the Navier-Stokes equation accounting for the back reaction of the solenoidal magnetic field on the flow:

$$
\begin{gathered}
\frac{\partial \mathbf{B}}{\partial t}=\boldsymbol{\nabla} \times(\mathbf{u} \times \mathbf{B})+\eta \boldsymbol{\nabla}^{2} \mathbf{B}, \\
\rho\left(\frac{\partial \mathbf{u}}{\partial t}+\mathbf{u} \cdot \boldsymbol{\nabla u}\right)=-\nabla P-2 \rho(\boldsymbol{\Omega} \times \mathbf{u})+\rho \nu \nabla^{2} \mathbf{u}+\frac{1}{\mu_{0}}(\boldsymbol{\nabla} \times \mathbf{B}) \times \mathbf{B}+\mathbf{g} C, \\
\boldsymbol{\nabla} \cdot \mathbf{B}=0 .
\end{gathered}
$$

This set of equations is solved in the planetary reference frame and gravitational acceleration $\mathbf{g}$ is directed along $\mathbf{e}_{\mathrm{r}}$. The magnetic diffusivity of the fluid is defined as $\eta$ and its magnetic permeability as $\mu_{0}$.

We adopt stress-free conditions at both ICB and CMB, to mitigate the influence of viscosity on the fluid outer core boundaries. The viscosity is the least realistic parameter in numerical dynamo models, being overestimated by several orders of magnitude. This condition can be written

$$
\left.u_{r}\right|_{r=r_{\mathrm{i}}, r_{\mathrm{o}}}=0
$$

and

$$
\left.\frac{\partial}{\partial r}\left(\frac{u_{\theta}}{r}\right)\right|_{r=r_{\mathrm{i}}, r_{\mathrm{o}}}=\left.\frac{\partial}{\partial r}\left(\frac{u_{\varphi}}{r}\right)\right|_{r=r_{\mathrm{i}}, r_{\mathrm{o}}}=0 .
$$

The mass anomaly flux $F$ is spatially homogeneous and remains constant over time at the inner core surface $S_{\mathrm{ICB}}$ and is taken as zero at the CMB so that

$$
\begin{aligned}
F & =\int_{S_{\mathrm{ICB}}} \kappa \nabla C \cdot \mathrm{dS}, \text { and } \\
0 & =\int_{S_{\mathrm{CMB}}} \kappa \boldsymbol{\nabla} C \cdot \mathbf{d} \mathbf{S},
\end{aligned}
$$

with $S_{\mathrm{CMB}}$ the core-mantle boundary surface. This simulates a situation where convection is entirely bottom-driven, as would be the case if the CMB total heat flux were exactly adiabatic. The inner core is modeled as a rigid body, free to rotate at an angular velocity $\Omega_{\mathrm{ic}}$ under the influence of magnetic and gravitational torques. We assume that the electrical conductivities of the fluid and the solid parts of the core are equal, and we denote them by $\sigma_{\mathrm{c}}$. This greatly simplifies our model and appears a reasonable assumption in light of mineral physics estimates (Pozzo et al., 2012; de Koker et al., 2012; Gomi et al., 2013). The mantle is considered as conductive in a layer of thickness $\Delta$ and conductivity $\sigma_{\mathrm{m}}$ directly above the CMB. At radii greater than $r_{\mathrm{o}}+\Delta$, the mantle is considered as insulating and the magnetic field is a potential field.

The angular momentum evolution in both inner core and mantle is determined by the 
torque balance between electromagnetic and gravitational coupling

$$
\begin{aligned}
\mathrm{I}_{\mathrm{m}} \frac{\mathrm{d} \Omega_{\mathrm{m}}}{\mathrm{dt}} & =-\Gamma_{\mathrm{G}}+\Gamma_{\mathrm{CMB}} \\
\mathrm{I}_{\mathrm{i}} \frac{\mathrm{d} \Omega_{\mathrm{ic}}}{\mathrm{dt}} & =\Gamma_{\mathrm{G}}+\Gamma_{\mathrm{ICB}}
\end{aligned}
$$

with $I_{m}$ and $I_{i}$ respectively the moments of inertia of the mantle and the inner core. For the fluid outer core, the evolution of angular momentum can be written

$$
\frac{\mathrm{d}}{\mathrm{dt}} \int_{V} \rho(r \sin \theta)^{2} \omega_{\mathrm{f}} \mathrm{d} V=-\Gamma_{\mathrm{CMB}}-\Gamma_{\mathrm{ICB}}
$$

where $V$ is the volume of the outer core and $\omega_{\mathrm{f}}$ the local rotation rate of fluid parcels. The electromagnetic torques acting on the mantle and the inner core, respectively $\Gamma_{\mathrm{CMB}}$ and $\Gamma_{\mathrm{ICB}}$, can be evaluated following the formalism developed by Rochester $(1960,1962)$. The moment of the Lorentz force integrated over a given volume is thereby reduced to the integral of the product of the radial and azimuthal magnetic field, $B_{r}$ and $B_{\varphi}$, over the surface of the core-mantle boundary for $\Gamma_{\mathrm{CMB}}$ and the surface of the inner core for $\Gamma_{\mathrm{ICB}}$. The magnetic torques can then be written

$$
\begin{aligned}
\Gamma_{\mathrm{CMB}} & =-\frac{r_{\mathrm{o}}}{\mu_{0}} \int_{S_{\mathrm{CMB}}} B_{r} B_{\varphi} \sin \theta \mathrm{dS}, \text { and } \\
\Gamma_{\mathrm{ICB}} & =\frac{r_{\mathrm{i}}}{\mu_{0}} \int_{S_{\mathrm{ICB}}} B_{r} B_{\varphi} \sin \theta \mathrm{dS}
\end{aligned}
$$

respectively. The restoring gravitational torque $\Gamma_{G}$ exerted on the inner core is proportional to its misalignment angle $\phi$ relative to the mantle, and a gravitational coupling constant $\Gamma$ (Buffett, 1997; Dumberry, 2007) as

$$
\Gamma_{\mathrm{G}}=-\phi \Gamma
$$

At any given time, the evolution of this misalignment angle is governed by

$$
\frac{\mathrm{d} \phi}{\mathrm{d} t}=\Omega_{\mathrm{ic}}-\Omega_{\mathrm{m}}-\frac{\phi}{\tau},
$$

with $\Omega_{\text {ic }}$ and $\Omega_{\mathrm{m}}$, respectively the solid-body rotation rates of the inner core and the mantle, and $\tau$ the viscous relaxation time of the inner core. Our focus on the long-term behavior of the geodynamo in this study allows us to adopt a simpler expression for $\phi$. When the fluctuations of $\phi$ occur on time scales longer than the inner core relaxation time this gives $\mathrm{d} \phi / \mathrm{d} t \ll \phi / \tau$, and thus $\phi=\left(\Omega_{\mathrm{ic}}-\Omega_{\mathrm{m}}\right) \tau$ (Aubert and Dumberry, 2011). Introducing this expression into (4.16) leads to

$$
\Gamma_{\mathrm{G}}=-\left(\Omega_{\mathrm{ic}}-\Omega_{\mathrm{m}}\right) \tau \Gamma
$$




\subsubsection{Dimensionless equations and numerical implementation}

Since our study relies on the rotational dynamics of the geodynamo we choose $\Omega^{-1}$, the inverse of the planetary rotation rate, as the relevant timescale. The length scale is $D$, the thickness of the fluid shell. Magnetic induction $B$ is scaled by $\left(\rho \mu_{0}\right)^{1 / 2} \Omega D$ and the non-hydrostatic pressure by $\rho \Omega^{2} D^{2}$, following the study of Christensen and Aubert (2006). Finally, the codensity $C$ is scaled with $F / 4 \pi D^{3} \Omega$ (Aubert et al., 2009). For simplicity, we adopt in the following sections the same notation for dimensionless variables as for our previously defined dimensional variables. From herein, equations and results will be presented in dimensionless form. Governing equations can then be written as

$$
\begin{aligned}
\frac{\partial \mathbf{u}}{\partial t}+\mathbf{u} \cdot \boldsymbol{\nabla} \mathbf{u}+\boldsymbol{\nabla} P+2 \mathbf{e}_{\mathbf{z}} \times \mathbf{u} & =E \nabla^{2} \mathbf{u}+(\boldsymbol{\nabla} \times \mathbf{B}) \times \mathbf{B}+\frac{\mathbf{r}}{r_{\mathrm{o}}} \mathcal{R} a_{F} C \\
\frac{\partial C}{\partial t}+\mathbf{u} \cdot \boldsymbol{\nabla} C & =E_{\kappa} \boldsymbol{\nabla}^{2} C+S_{T / \xi} \\
\frac{\partial \mathbf{B}}{\partial t}-\boldsymbol{\nabla} \times(\mathbf{u} \times \mathbf{B}) & =E_{\eta} \boldsymbol{\nabla}^{2} \mathbf{B}
\end{aligned}
$$

The four non-dimensional parameters are the Ekman number,

$$
E=\frac{\nu}{\Omega D^{2}}
$$

the thermochemical Ekman number,

$$
E_{\kappa}=\frac{\kappa}{\Omega D^{2}}
$$

the magnetic Ekman number,

$$
E_{\eta}=\frac{\eta}{\Omega D^{2}}
$$

and the modified Rayleigh number,

$$
\mathcal{R} a_{F}=\frac{g_{\mathrm{o}} F}{4 \pi \rho \Omega^{3} D^{4}}
$$

accounting for a fixed-flux boundary condition at the inner core surface, with $g_{\mathrm{o}}$ the gravitational acceleration at the CMB. Values of the input parameters for the 45 models considered in this study are summarized in tables 4.1 and 4.2.

In dimensionless form, equations (4.11) and (4.12) now become

$$
\begin{aligned}
\mathrm{I}_{\mathrm{m}} \frac{\mathrm{d} \Omega_{\mathrm{m}}}{\mathrm{dt}} & =\zeta\left(\Omega_{\mathrm{ic}}-\Omega_{\mathrm{m}}\right)+\frac{\Gamma_{\mathrm{CMB}}}{\rho D^{5} \Omega^{2}}, \\
\mathrm{I}_{\mathrm{i}} \frac{\mathrm{d} \Omega_{\mathrm{ic}}}{\mathrm{dt}} & =-\zeta\left(\Omega_{\mathrm{ic}}-\Omega_{\mathrm{m}}\right)+\frac{\Gamma_{\mathrm{ICB}}}{\rho D^{5} \Omega^{2}}
\end{aligned}
$$


with $\Gamma_{\mathrm{CMB}}$ and $\Gamma_{\mathrm{ICB}}$ as defined by equations (4.14) and (4.15). This system includes a new dimensionless parameter, $\zeta$, representing the strength of the gravitational coupling between the inner core and the mantle. As moments of inertia are scaled using $\rho D^{5}$ and electromagnetic torques using $\rho D^{5} \Omega^{2}$, this gives

$$
\zeta=\frac{\tau \Gamma}{\rho D^{5} \Omega}
$$

Given our choice of characteristic scales, $\zeta$ is normalized by the fluid core angular momentum, it thus compares the relative importance of gravitational coupling and core inertia.

Finally, it is important to mention one last dimensionless parameter that does not directly appear in the equations but which enters the formulation of the CMB magnetic boundary condition, namely the parameter

$$
\Sigma=\frac{\Delta \sigma_{\mathrm{m}}}{D \sigma_{\mathrm{c}}}
$$

The parameter $\Sigma$ compares the relative importance of mantle and core conductances. It should be kept in mind that while values of $\zeta$ of $\mathcal{O}(1)$ are geophysically admissible, values of $\Sigma$ of the same order are clearly unrealistic such that in general $\Sigma \ll 1$. The mantle is a poor electrical conductor (see e.g. Civet et al., 2015, and references therein), at the exception (already discussed in the introduction) of a thin layer above the CMB (Ohta et al., 2008, 2012) whose conductance may reach the value of $10^{8} \mathrm{~S}$ inferred by Buffett (1992). This last value is four orders of magnitude lower than the fluid core conductance. We can therefore anticipate that the geophysically relevant range for $\Sigma$ does not extend beyond $10^{-3}$. For the sake of completeness, however, the range we shall consider in this study covers 7 orders of magnitude, with $\Sigma$ varying from $10^{-8}$ to $10^{-1}$.

We performed numerical 45 simulations of the system (4.19)-(4.29) using the PARODYJA numerical implementation (Dormy et al., 1998; Aubert et al., 2008), the latest version of which uses the spectral transform library SHTns (Schaeffer, 2013). Tables 4.1 and 4.2 summarize the input and output parameters of the parameter space survey. Among the outputs not yet defined, we mention here: $A D /(A D+N A D)$, the relative axial dipole power defined by Christensen et al. (2010), $L_{\max }$, the maximum degree and order of the spherical harmonic decomposition in the horizontal directions, the number of grid points in the radial direction $N R$, and the mean squared magnetic field respectively at ICB and CMB, $B_{\mathrm{r}_{\mathrm{i}}}^{2}$ and $B_{\mathrm{r}_{\mathrm{o}}}^{2}$. Most dynamos exhibit a strong dipolar component, i.e $A D /(A D+N A D) \geq 0.6$. Since we are mainly interested in the long-term (time-average) behavior of the system, simulations were time-integrated as to ensure convergence of time-averages for the values of interest (see tables 4.1 and 4.2). 


\begin{tabular}{|c|c|c|c|c|c|c|c|c|c|c|c|c|}
\hline & $\mathcal{R} a_{F}$ & $\Sigma$ & $\zeta$ & $\mathcal{D}$ & $\mathcal{C}_{\mathrm{f}}$ & $\mathcal{C}_{\text {icb }}$ & $\mathcal{S}$ & $B_{\mathrm{r}_{\mathrm{i}}}^{2}$ & $B_{\mathrm{r}_{\mathrm{o}}}^{2}$ & $\frac{A D}{A D+N A D}$ & $N R$ & $L_{\max }$ \\
\hline & $2.70 \times 10^{-5}$ & $1.00 \times 10^{-8}$ & 0.75 & $-5.30 \times 10^{-3}$ & 0.01 & $5.02 \times 10^{-3}$ & $2.76 \times 10^{-6}$ & $4.40 \times 10^{-4}$ & $1.01 \times 10^{-5}$ & 0.6 & 120 & 85 \\
\hline & $2.70 \times 10^{-5}$ & $1.00 \times 10^{-7}$ & 0.75 & $-5.04 \times 10^{-3}$ & 0.01 & $5.20 \times 10^{-3}$ & $2.78 \times 10^{-6}$ & $4.59 \times 10^{-4}$ & $9.42 \times 10^{-6}$ & 0.65 & 120 & 85 \\
\hline & $2.70 \times 10^{-5}$ & $1.00 \times 10^{-6}$ & 0.75 & $-5.49 \times 10^{-3}$ & 0.01 & $4.75 \times 10^{-3}$ & $2.77 \times 10^{-6}$ & $4.65 \times 10^{-4}$ & $1.04 \times 10^{-5}$ & 0.67 & 120 & 85 \\
\hline & $2.70 \times 10^{-5}$ & $1.00 \times 10^{-5}$ & 0.75 & $-5.27 \times 10^{-3}$ & 0.01 & $5.27 \times 10^{-3}$ & $3.54 \times 10^{-6}$ & $4.72 \times 10^{-4}$ & $1.01 \times 10^{-5}$ & 0.66 & 120 & 85 \\
\hline & $2.70 \times 10^{-5}$ & $5.00 \times 10^{-5}$ & 0.75 & $-4.36 \times 10^{-3}$ & 0.01 & $5.76 \times 10^{-3}$ & $5.75 \times 10^{-6}$ & $4.62 \times 10^{-4}$ & $9.75 \times 10^{-6}$ & 0.65 & 120 & 85 \\
\hline & $2.70 \times 10^{-5}$ & $1.00 \times 10^{-4}$ & 0.75 & $-3.99 \times 10^{-3}$ & $9.93 \times 10^{-3}$ & $5.93 \times 10^{-3}$ & $8.82 \times 10^{-6}$ & $4.51 \times 10^{-4}$ & $1.07 \times 10^{-5}$ & 0.67 & 120 & 85 \\
\hline & $\begin{array}{l}2.70 \times 10^{-5} \\
2.70 \times 10^{-1}\end{array}$ & $\begin{array}{l}1.00 \times 10 \\
1.00 \times 10^{-3}\end{array}$ & $\begin{array}{l}0.75 \\
0.75\end{array}$ & $\begin{array}{l}-3.99 \times 10^{-3} \\
-1.50 \times 10^{-3}\end{array}$ & $\begin{array}{l}9.93 \times 10^{-3} \\
9.41 \times 10^{2}\end{array}$ & $\begin{array}{l}5.93 \times 10^{-3} \\
7.89 \times 10^{-1}\end{array}$ & $\begin{array}{l}8.82 \times 10^{-5} \\
1.97 \times 10^{-1}\end{array}$ & $\begin{array}{l}4.51 \times 10 \\
4.75 \times 10^{-4}\end{array}$ & $1.39 \times 10^{-5}$ & $\begin{array}{l}0.67 \\
0.63\end{array}$ & $\begin{array}{l}120 \\
120\end{array}$ & $\begin{array}{l}85 \\
85\end{array}$ \\
\hline & $2.70 \times 10^{-5}$ & $5.00 \times 10^{-3}$ & 0.75 & $-8.17 \times 10^{-4}$ & 0.01 & $9.22 \times 10^{-3}$ & $2.49 \times 10^{-5}$ & $4.54 \times 10^{-4}$ & $6.19 \times 10^{-5}$ & 0.58 & 120 & $\begin{array}{l}85 \\
85\end{array}$ \\
\hline & $2.70 \times 10^{-5}$ & 0.01 & 0.75 & $-7.58 \times 10^{-4}$ & 0.01 & 0.01 & $2.29 \times 10^{-5}$ & $3.95 \times 10^{-4}$ & $1.21 \times 10^{-4}$ & 0.50 & 120 & 85 \\
\hline & $2.70 \times 10^{-5}$ & 0.05 & 0.75 & $-4.36 \times 10^{-4}$ & 0.01 & 0.01 & $2.09 \times 10^{-5}$ & $3.22 \times 10^{-4}$ & $4.38 \times 10^{-4}$ & 0.21 & 120 & 85 \\
\hline (D) & $2.70 \times 10^{-5}$ & 0.10 & 0.75 & $-2.10 \times 10^{-4}$ & 0.01 & 0.01 & $1.75 \times 10^{-5}$ & $3.08 \times 10^{-4}$ & $5.64 \times 10^{-4}$ & 0.05 & 120 & 85 \\
\hline & $2.70 \times 10^{-5}$ & $1.00 \times 10^{-4}$ & 0 & $1.23 \times 10^{-3}$ & 0.01 & $4.22 \times 10^{-3}$ & $7.40 \times 10^{-3}$ & $4.66 \times 10^{-4}$ & $1.03 \times 10^{-5}$ & 0.67 & 120 & $\begin{array}{l}80 \\
85\end{array}$ \\
\hline & $2.70 \times 10^{-5}$ & $1.00 \times 10^{-4}$ & $3.00 \times 10^{-4}$ & $-7.27 \times 10^{-5}$ & $\begin{array}{l}0.01 \\
0.01\end{array}$ & $4.84 \times 10^{-3}$ & $5.49 \times 10^{-3}$ & $4.55 \times 10^{-4}$ & $9.89 \times 10^{-6}$ & $\begin{array}{l}0.01 \\
0.66\end{array}$ & $\begin{array}{l}120 \\
120\end{array}$ & $\begin{array}{l}00 \\
85\end{array}$ \\
\hline & $2.70 \times 10^{-5}$ & $1.00 \times 10^{-4}$ & $1.50 \times 10^{-3}$ & $-2.02 \times 10^{-3}$ & 0.01 & $5.57 \times 10^{-3}$ & $2.75 \times 10^{-3}$ & $4.84 \times 10^{-4}$ & $1.05 \times 10^{-5}$ & 0.66 & 120 & 85 \\
\hline & $2.70 \times 10^{-5}$ & $1.00 \times 10^{-4}$ & $3.00 \times 10^{-3}$ & $-2.78 \times 10^{-3}$ & 0.01 & $5.66 \times 10^{-3}$ & $1.67 \times 10^{-3}$ & $4.85 \times 10^{-4}$ & $1.04 \times 10^{-5}$ & 0.66 & 120 & 85 \\
\hline & $2.70 \times 10^{-5}$ & $1.00 \times 10^{-4}$ & $7.50 \times 10^{-3}$ & $-3.31 \times 10^{-3}$ & $\begin{array}{l}0.01 \\
0.01\end{array}$ & $5.96 \times 10^{-3}$ & $7.45 \times 10^{-4}$ & $4.60 \times 10^{-4}$ & $\begin{array}{l}1.04 \times 10^{-5} \\
1.02 \times 10^{-0}\end{array}$ & $\begin{array}{l}0.66 \\
0.66\end{array}$ & $\begin{array}{l}120 \\
120\end{array}$ & $\begin{array}{l}85 \\
85\end{array}$ \\
\hline & $2.70 \times 10^{-5}$ & $1.00 \times 10^{-4}$ & $\begin{array}{l}.50 \times 102 \\
0.02\end{array}$ & $-3.63 \times 10^{-3}$ & $\begin{array}{l}0.01 \\
0.01\end{array}$ & $6.33 \times 10^{-3}$ & $3.88 \times 10^{-4}$ & $4.51 \times 10^{-4}$ & $9.75 \times 10^{-6}$ & $\begin{array}{l}0.66 \\
0.64\end{array}$ & $\begin{array}{l}120 \\
120\end{array}$ & $\begin{array}{l}85 \\
85\end{array}$ \\
\hline & $2.70 \times 10^{-5}$ & $1.00 \times 10^{-4}$ & $\begin{array}{l}0.02 \\
0.03\end{array}$ & $-3.62 \times 10^{-3}$ & $\begin{array}{l}0.01 \\
0.01\end{array}$ & $6.19 \times 10^{-3}$ & $1.93 \times 10^{-4}$ & $4.53 \times 10^{-4}$ & $9.70 \times 10^{-6}$ & $\begin{array}{l}0.04 \\
0.65\end{array}$ & 120 & $\begin{array}{l}85 \\
85\end{array}$ \\
\hline & $2.70 \times 10^{-5}$ & $1.00 \times 10^{-4}$ & 0.05 & $-3.80 \times 10^{-3}$ & 0.01 & $6.08 \times 10^{-3}$ & $1.20 \times 10^{-4}$ & $5.01 \times 10^{-4}$ & $1.05 \times 10^{-5}$ & 0.66 & 120 & 85 \\
\hline & $2.70 \times 10^{-5}$ & $1.00 \times 10^{-4}$ & 0.07 & $-3.77 \times 10^{-3}$ & $9.87 \times 10^{-3}$ & $6.01 \times 10^{-3}$ & $8.23 \times 10^{-5}$ & $4.57 \times 10^{-4}$ & $1.02 \times 10^{-5}$ & $\begin{array}{l}0.00 \\
0.65\end{array}$ & 120 & $\begin{array}{l}80 \\
85\end{array}$ \\
\hline & $\begin{array}{l}2.70 \times 10^{-5} \\
2.70 \times 10^{-1}\end{array}$ & $\begin{array}{l}1.00 \times 10^{-4} \\
1.00 \times 10^{-1}\end{array}$ & $\begin{array}{l}0.07 \\
0.15\end{array}$ & $-3.90 \times 10^{-3}$ & $\begin{array}{c}9.87 \times 10 \\
0.01\end{array}$ & $\begin{array}{l}6.01 \times 10^{-3} \\
6.21 \times 10^{2}\end{array}$ & $\begin{array}{l}8.23 \times 10^{-5} \\
4.16 \times 10^{-1}\end{array}$ & $\begin{array}{l}4.57 \times 10 \\
4.70 \times 10^{-4}\end{array}$ & $\begin{array}{l}1.02 \times 10^{-6} \\
9.92 \times 10^{-1}\end{array}$ & $\begin{array}{l}0.65 \\
0.66\end{array}$ & $\begin{array}{l}120 \\
120\end{array}$ & $\begin{array}{l}85 \\
85\end{array}$ \\
\hline & $2.70 \times 10^{-5}$ & $1.00 \times 10^{-4}$ & $\begin{array}{l}0.15 \\
0.30\end{array}$ & $-3.89 \times 10^{-3}$ & $\begin{array}{l}0.01 \\
0.01\end{array}$ & $\begin{array}{l}6.21 \times 10^{-3} \\
6.29 \times 10^{2}\end{array}$ & $\begin{array}{l}4.10 \times 10^{-5} \\
2.06 \times 10^{-1}\end{array}$ & $4.20 \times 10^{-4}$ & $9.92 \times 10^{-6}$ & $\begin{array}{l}0.66 \\
0.65\end{array}$ & $\begin{array}{l}120 \\
120\end{array}$ & $\begin{array}{l}85 \\
85\end{array}$ \\
\hline & $2.70 \times 10^{-5}$ & $1.00 \times 10^{-4}$ & 0.45 & $-3.75 \times 10^{-3}$ & $9.99 \times 10^{-3}$ & $6.24 \times 10^{-3}$ & $1.30 \times 10^{-5}$ & $4.78 \times 10^{-4}$ & $9.66 \times 10^{-6}$ & 0.64 & 120 & 85 \\
\hline & $2.70 \times 10^{-5}$ & $1.00 \times 10^{-4}$ & 0.60 & $-3.91 \times 10^{-3}$ & 0.01 & $6.34 \times 10^{-3}$ & $1.03 \times 10^{-5}$ & $4.35 \times 10^{-4}$ & $9.90 \times 10^{-6}$ & 0.65 & 120 & 85 \\
\hline & $9.00 \times 10^{-6}$ & $1.00 \times 10^{-4}$ & $3.00 \times 10^{-3}$ & $-2.34 \times 10^{-3}$ & $5.97 \times 10^{-3}$ & $2.54 \times 10^{-3}$ & $1.09 \times 10^{-3}$ & $3.75 \times 10^{-4}$ & $9.90 \times 10^{-6}$ & 0.79 & 120 & 85 \\
\hline & $\begin{array}{l}9.00 \times 10^{-5} \\
1.35 \times 10^{-1}\end{array}$ & $\begin{array}{l}1.00 \times 10^{-4} \\
1.00 \times 10^{-1}\end{array}$ & $\begin{array}{l}3.00 \times 10 \\
3.00 \times 10^{-3}\end{array}$ & $\begin{array}{l}-2.34 \times 10 \\
-2.63 \times 10^{-3}\end{array}$ & $7.72 \times 10^{-3}$ & $\begin{array}{l}2.54 \times 10 \\
3.79 \times 10^{-3}\end{array}$ & $\begin{array}{l}1.09 \times 10^{-3} \\
1.29 \times 10^{-1}\end{array}$ & $\begin{array}{l}3.55 \times 10^{-4} \\
4.13 \times 10^{-1}\end{array}$ & $\begin{array}{l}9.90 \times 10^{-5} \\
1.03 \times 10^{-1}\end{array}$ & $\begin{array}{l}0.79 \\
0.74\end{array}$ & $\begin{array}{l}120 \\
120\end{array}$ & $\begin{array}{l}85 \\
85\end{array}$ \\
\hline & $1.80 \times 10^{-5}$ & $1.00 \times 10^{-4}$ & $3.00 \times 10^{-3}$ & $-2.80 \times 10^{-3}$ & $8.88 \times 10^{-3}$ & $4.67 \times 10^{-3}$ & $1.41 \times 10^{-3}$ & $4.32 \times 10^{-4}$ & $1.01 \times 10^{-5}$ & $\begin{array}{l}0.74 \\
0.71\end{array}$ & $\begin{array}{l}120 \\
120\end{array}$ & $\begin{array}{l}85 \\
85\end{array}$ \\
\hline & $2.25 \times 10^{-5}$ & $1.00 \times 10^{-4}$ & $3.00 \times 10^{-3}$ & $-2.75 \times 10^{-3}$ & $9.58 \times 10^{-3}$ & $5.31 \times 10^{-3}$ & $1.52 \times 10^{-3}$ & $4.55 \times 10^{-4}$ & $1.03 \times 10^{-5}$ & 0.68 & 120 & 85 \\
\hline (A) & $2.70 \times 10^{-5}$ & $1.00 \times 10^{-4}$ & 0 & $-5.68 \times 10^{-5}$ & 0.01 & $4.14 \times 10^{-3}$ & $6.41 \times 10^{-3}$ & $4.82 \times 10^{-4}$ & $1.05 \times 10^{-5}$ & $\begin{array}{l}0.00 \\
0.68\end{array}$ & 220 & 85 \\
\hline & $2.25 \times 10^{-5}$ & $1.00 \times 10^{-4}$ & 0 & $-1.55 \times 10^{-4}$ & $9.95 \times 10^{-3}$ & $3.91 \times 10^{-3}$ & $5.88 \times 10^{-3}$ & $4.79 \times 10^{-4}$ & $1.08 \times 10^{-5}$ & 0.71 & 220 & 85 \\
\hline & $1.80 \times 10^{-5}$ & $1.00 \times 10^{-4}$ & 0 & $-1.78 \times 10^{-4}$ & $8.91 \times 10^{-3}$ & $3.29 \times 10^{-3}$ & $5.45 \times 10^{-3}$ & $4.65 \times 10^{-4}$ & $1.11 \times 10^{-5}$ & 0.73 & 220 & $\begin{array}{l}80 \\
85\end{array}$ \\
\hline & $1.35 \times 10^{-5}$ & $1.00 \times 10^{-4}$ & 0 & $-2.10 \times 10^{-4}$ & $7.67 \times 10^{-3}$ & $2.77 \times 10^{-3}$ & $4.69 \times 10^{-3}$ & $4.36 \times 10^{-4}$ & $1.09 \times 10^{-5}$ & 0.76 & 220 & $\begin{array}{l}00 \\
85\end{array}$ \\
\hline & $9.00 \times 10^{-6}$ & $1.00 \times 10^{-4}$ & 0 & $-2.67 \times 10^{-4}$ & $6.03 \times 10^{-3}$ & $1.93 \times 10^{-3}$ & $3.84 \times 10^{-3}$ & $3.84 \times 10^{-4}$ & $1.02 \times 10^{-5}$ & 0.79 & 220 & 85 \\
\hline & $6.30 \times 10^{-5}$ & $1.00 \times 10^{-4}$ & 0 & $1.50 \times 10^{-3}$ & $8.39 \times 10^{-3}$ & $3.11 \times 10^{-3}$ & $6.78 \times 10^{-3}$ & $6.21 \times 10^{-4}$ & $1.29 \times 10^{-5}$ & 0.62 & 220 & 85 \\
\hline & $\begin{array}{l}6.30 \times 10^{-5} \\
7.20 \times 10^{-1}\end{array}$ & $1.00 \times 10^{-4}$ & 0 & $1.96 \times 10^{-3}$ & $\begin{array}{l}8.39 \times 10^{-3} \\
5.95 \times 10^{2}\end{array}$ & $\begin{array}{l}3.11 \times 10^{-3} \\
1.95 \times 10^{-1}\end{array}$ & $\begin{array}{l}6.78 \times 10^{-3} \\
5.9510^{2}\end{array}$ & $\begin{array}{l}6.21 \times 10 \\
6.97 \times 10^{-4}\end{array}$ & $1.49 \times 10^{-5}$ & $\begin{array}{l}0.62 \\
0.65\end{array}$ & $\begin{array}{l}220 \\
220\end{array}$ & $\begin{array}{l}85 \\
85\end{array}$ \\
\hline & $2.70 \times 10^{-5}$ & $1.00 \times 10^{-4}$ & 0.75 & $\begin{array}{l}1.96 \times 10^{-3} \\
-4.14 \times 10^{-1}\end{array}$ & $\begin{array}{c}5.95 \times 10 \\
0.01\end{array}$ & $\begin{array}{l}1.95 \times 10^{-3} \\
6.22 \times 10^{-1}\end{array}$ & $\begin{array}{l}5.95 \times 10^{-6} \\
7.45\end{array}$ & $4.57 \times 10^{-4}$ & $9.69 \times 10^{-6}$ & $\begin{array}{l}0.65 \\
0.65\end{array}$ & $\begin{array}{l}220 \\
140\end{array}$ & $\begin{array}{l}85 \\
85\end{array}$ \\
\hline & $2.70 \times 10^{-5}$ & $1.00 \times 10^{-4}$ & $\begin{array}{l}0.15 \\
0.75\end{array}$ & $\begin{array}{l}-4.14 \times 10 \\
-4.25 \times 10\end{array}$ & $9.95 \times 10^{-3}$ & $\begin{array}{l}5.22 \times 10 \\
5.69 \times 10\end{array}$ & $\begin{array}{l}.45 \times 10 \\
7.97 \times 10\end{array}$ & $4.88 \times 10$ & $\begin{array}{l}.09 \times 10 \\
1.09 \times 10\end{array}$ & 0.67 & 160 & $\begin{array}{l}85 \\
85\end{array}$ \\
\hline & $2.70 \times 10^{-}$ & $1.00 \times 10^{-4}$ & 0.75 & $-4.33 \times 10$ & 0.01 & $5.78 \times 10^{-3}$ & $7.22 \times 10^{-6}$ & $4.76 \times 10^{-4}$ & $1.03 \times 10^{-5}$ & 0.67 & 180 & 85 \\
\hline & $2.70 \times 10^{-5}$ & $1.00 \times 10^{-4}$ & 0.75 & $-4.36 \times 10^{-3}$ & 0.01 & $5.69 \times 10^{-3}$ & $7.31 \times 10^{-6}$ & $4.79 \times 10^{-4}$ & $1.05 \times 10^{-5}$ & 0.67 & 200 & 85 \\
\hline & $2.70 \times 10^{-5}$ & $1.00 \times 10^{-4}$ & 0.75 & $-4.40 \times 10^{-3}$ & 0.01 & $5.94 \times 10^{-3}$ & $6.91 \times 10^{-6}$ & $4.76 \times 10^{-4}$ & $1.03 \times 10^{-5}$ & $\begin{array}{l}0.06 \\
0.66\end{array}$ & 220 & $\begin{array}{l}80 \\
85\end{array}$ \\
\hline & $2.70 \times 10^{-5}$ & $1.00 \times 10^{-4}$ & 0.75 & $-4.40 \times 10^{-3}$ & $\begin{array}{l}0.01 \\
0.01\end{array}$ & $5.88 \times 10^{-3}$ & $6.48 \times 10^{-6}$ & $4.68 \times 10^{-4}$ & $9.84 \times 10^{-6}$ & $\begin{array}{l}0.00 \\
0.66\end{array}$ & 240 & $\begin{array}{l}85 \\
85\end{array}$ \\
\hline (B) & $2.70 \times 10^{-5}$ & $1.00 \times 10^{-8}$ & 0.75 & $-5.92 \times 10^{-3}$ & 0.01 & $4.72 \times 10^{-3}$ & $4.93 \times 10^{-7}$ & $4.59 \times 10^{-4}$ & $9.70 \times 10^{-6}$ & $\begin{array}{l}0.00 \\
0.67\end{array}$ & 220 & 85 \\
\hline
\end{tabular}

Table 4.1: Parameters of the numerical simulations. All runs were performed with $E=E_{\kappa}=3 \times 10^{-5}$ and $E_{\eta}=1.2 \times 10^{-5}$. Labels A, B, C and D correspond to numerical simulations displayed in figures 4.2 and 4.3. 


\begin{tabular}{|c|c|c|c|c|c|c|c|c|c|}
\hline$E_{\eta}$ & $\mathcal{R} a_{F}$ & $\mathcal{D}$ & $\mathcal{C}_{\mathrm{f}}$ & $\mathcal{C}_{\mathrm{icb}}$ & $\mathcal{S}$ & $B_{\mathrm{r}_{\mathrm{i}}}^{2}$ & $B_{\mathrm{r}_{\mathrm{o}}}^{2}$ & $\frac{A D}{A D+N A D}$ & $L_{\max }$ \\
\hline $4.00 \times 10^{-6}$ & $3.00 \times 10^{-6}$ & $2.06 \times 10^{-5}$ & $3.50 \times 10^{-3}$ & $1.72 \times 10^{-3}$ & $1.80 \times 10^{-3}$ & $2.34 \times 10^{-4}$ & $7.07 \times 10^{-6}$ & 0.70 & 133 \\
\hline $6.67 \times 10^{-6}$ & $9.00 \times 10^{-6}$ & $-2.59 \times 10^{-4}$ & $7.47 \times 10^{-3}$ & $3.42 \times 10^{-3}$ & $3.80 \times 10^{-3}$ & $2.24 \times 10^{-4}$ & $4.71 \times 10^{-6}$ & 0.64 & 170 \\
\hline $6.67 \times 10^{-6}$ & $2.00 \times 10^{-5}$ & $7.47 \times 10^{-4}$ & $7.69 \times 10^{-3}$ & $3.89 \times 10^{-3}$ & $4.55 \times 10^{-3}$ & $2.19 \times 10^{-4}$ & $2.69 \times 10^{-6}$ & 0.45 & 170 \\
\hline
\end{tabular}

Table 4.2: Parameters of the numerical simulations with $E=E_{\kappa}=3 \times 10^{-5}$. Simulations have $\Sigma=1 \times 10^{-4}, \zeta=0$ and $N R=240$.

\subsubsection{Theoretical analysis of the long-term rotational state}

Before inspecting the results of our set of 45 simulations, we dedicate this section to the theoretical description of the long-term rotational dynamics of our system, the predictions of which will be tested against numerical results in section 4.3. As shown in figure 4.1, this long-term dynamics can be described using 4 rotation rates: $\Omega_{\mathrm{ic}}, \Omega_{\mathrm{m}}, \Omega_{\mathrm{ficb}}$ and $\Omega_{\mathrm{fcmb}}$, respectively the solid-body angular rotation rates of the inner core, the mantle, the fluid at the ICB and the fluid at the CMB. Recall indeed that stress-free boundary conditions allow for velocity jumps at the fluid outer core boundaries. At the ICB, this velocity jump is denoted as

$$
\mathcal{C}_{\text {icb }}=\left\langle\Omega_{\text {ficb }}-\Omega_{\text {ic }}\right\rangle
$$

the angle brackets \langle\rangle meaning time-average quantities. Similarly,

$$
\mathcal{D}=\left\langle\Omega_{\mathrm{fcmb}}-\Omega_{\mathrm{m}}\right\rangle
$$

denotes the equivalent at the CMB, the long-term geomagnetic westward drift. In addition, we define

$$
\mathcal{S}=\left\langle\Omega_{\text {ic }}-\Omega_{\mathrm{m}}\right\rangle
$$

the long-term inner core super-rotation, and

$$
\mathcal{C}_{\mathrm{f}}=\left\langle\Omega_{\mathrm{ficb}}-\Omega_{\mathrm{fcmb}}\right\rangle,
$$

the long-term global convective shear linking the two boundaries. The formal link between the four quantities is then

$$
\mathcal{C}_{\mathrm{f}}=\mathcal{C}_{\text {icb }}+\mathcal{S}-\mathcal{D}
$$

To derive the link between the long-term geomagnetic westward drift $\mathcal{D}$ and the longterm differential rotation of the inner core $\mathcal{S}$, the intuitive first step is then to relate the four components of the rotational dynamics expressed above to the strengths of the electromagnetic and gravitational torques (Eqs. 4.14, 4.15, 4.18), and use the fact that these torques should balance when considering the long-term conservation of angular momentum. Therefore, we approximate the time-average electromagnetic torques, $\left\langle\Gamma_{\mathrm{CMB}}\right\rangle$ and $\left\langle\Gamma_{\mathrm{ICB}}\right\rangle$, using the theoretical approach developed by Dumberry (2007). At the CMB, this theory relies on a thin layer approximation (Stewart et al., 1995; Holme, 1998) and the torque thus 


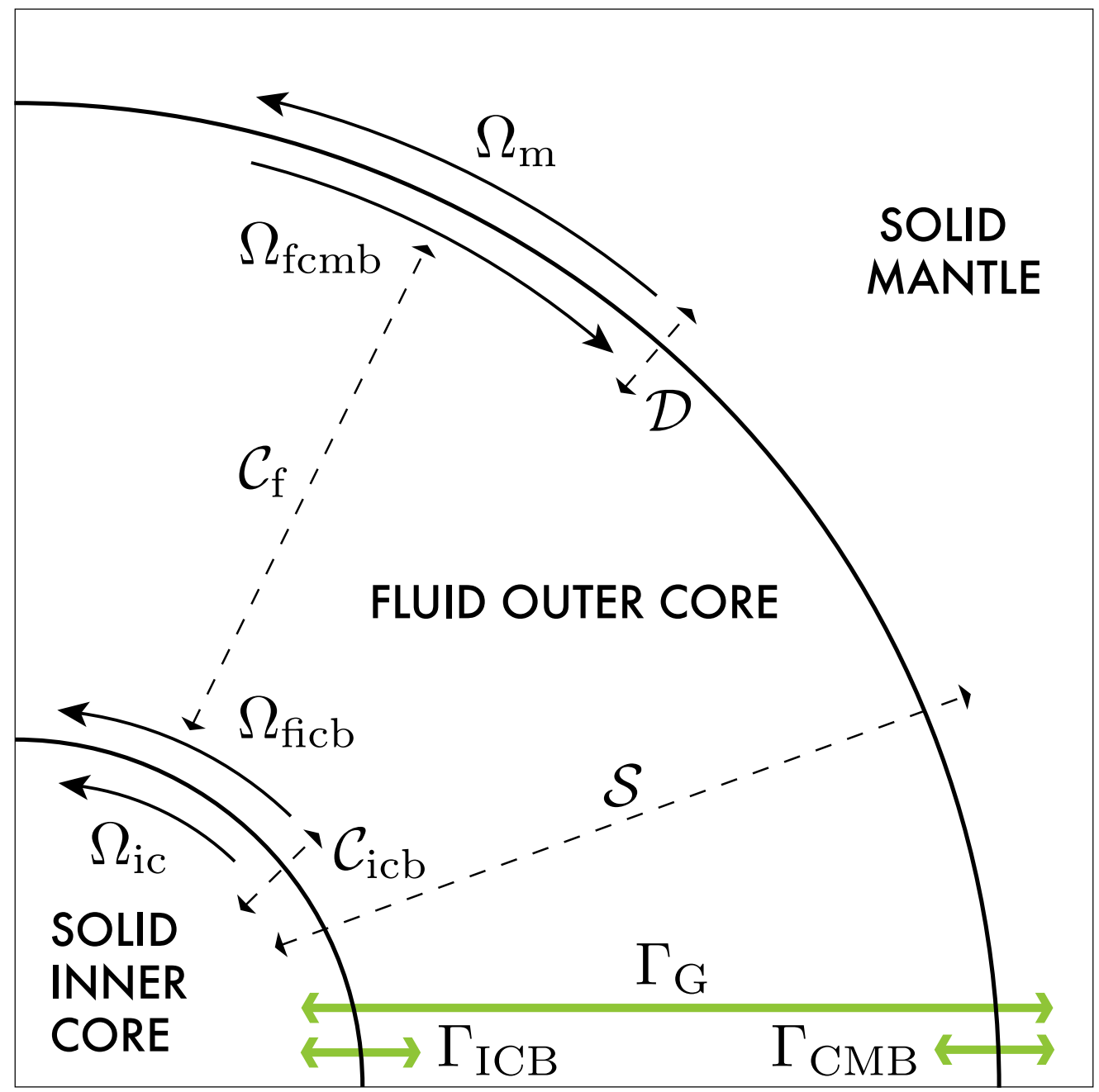

Figure 4.1: Rotational state of our system. Black arrowed lines define rotation rates: $\Omega_{\mathrm{m}}$ is the angular velocity of the solid mantle, $\Omega_{\mathrm{fcmb}}$ (resp. $\Omega_{\mathrm{ficb}}$ ) is the rotation rate of the fluid outer core in the vicinity of the CMB (resp. the ICB), and $\Omega_{\text {ic }}$ is the angular velocity of the solid inner core. Dashed lines represent the various time-average shears: $\mathcal{D}$ is the long-term westward drift (Eq. 4.31), $\mathcal{S}$ is the long-term inner core differential rotation (Eq. 4.32), $\mathcal{C}_{\mathrm{f}}$ is the long-term shear available in the fluid outer core (Eq. 4.33), and $\mathcal{C}_{\text {icb }}$ denotes the long-term shear at the ICB (Eq. 4.30). Green lines indicate torques. $\Gamma_{\mathrm{CMB}}\left(\mathrm{resp} . \Gamma_{\mathrm{ICB}}\right)$ is the electromagnetic torque at the CMB (resp. the ICB), and $\Gamma_{\mathrm{G}}$ is the gravitational torque exerted by the mantle on the inner core. 
results from a simple shear of the poloidal field by the westward drift. The torque is then directly proportional to the lower mantle conductance $\Delta \sigma_{\mathrm{m}}$, to the mean squared magnetic field at $\mathrm{CMB}\left(B_{\mathrm{r}_{\mathrm{o}}}^{2}\right)$ and to $\mathcal{D}$. In dimensional form this leads to

$$
\left\langle\Gamma_{\mathrm{CMB}}\right\rangle \simeq K_{1} r_{\mathrm{o}}^{4} B_{\mathrm{r}_{\mathrm{o}}}^{2} \Delta \sigma_{\mathrm{m}} \mathcal{D}
$$

with $K_{1}$ a numerical constant to be determined (see section 4.3.3). In dimensionless form this gives

$$
\left\langle\Gamma_{\mathrm{CMB}}\right\rangle \simeq \frac{K_{1}}{E_{\eta}} r_{\mathrm{o}}^{4} B_{\mathrm{r}_{\mathrm{o}}}^{2} \Sigma \mathcal{D} .
$$

In contrast to the situation at the CMB, modeling the electromagnetic torque at the ICB is more complex, in particular because the inner core is not a thin flat layer. Aurnou et al. $(1996,1998)$ highlighted that the consequence of this thick-layer configuration is that the azimuthal magnetic field initiating the torque is now made of two contributions: one due to the local shear at the ICB, and the other due to the shear in the tangent cylinder, resulting from the thermal wind balance. This idea was further explored by Dumberry (2007) through an analytical solution, allowing an estimate of the ratio between these two contributions from the shear at the ICB, $\mathcal{C}_{\mathrm{icb}}$, and in the fluid, $\mathcal{C}_{\mathrm{f}}$. This enables the formulation of the following dimensional model of the electromagnetic torque acting on the inner core,

$$
\left\langle\Gamma_{\mathrm{ICB}}\right\rangle \simeq K_{2} \sigma_{\mathrm{c}} r_{\mathrm{i}}^{5} B_{\mathrm{r}_{\mathrm{i}}}^{2}\left(\mathcal{C}_{\mathrm{icb}}-\alpha \mathcal{C}_{\mathrm{f}}\right)
$$

with $\alpha$ representing the relative amplitude between the two contributions and $K_{2}$ another numerical constant to be determined (see section 4.3.3). The dimensionless form of this model is then

$$
\left\langle\Gamma_{\mathrm{ICB}}\right\rangle \simeq \frac{K_{2}}{E_{\eta}} r_{\mathrm{i}}^{5} B_{\mathrm{r}_{\mathrm{i}}}^{2}\left(\mathcal{C}_{\mathrm{icb}}-\alpha \mathcal{C}_{\mathrm{f}}\right)
$$

Equation (4.38) reflects that in a situation where there is no restoring torque to balance $\left\langle\Gamma_{\text {ICB }}\right\rangle$, so that $\left\langle\Gamma_{\text {ICB }}\right\rangle=0$, the local and remote contribution to the electromagnetic torque cancel and

$$
\alpha=\frac{\mathcal{C}_{\text {icb }}}{\mathcal{C}_{\mathrm{f}}} .
$$

Finally, the time-average dimensionless gravitational torque exerted on the inner core is directly proportional to the long-term inner core super-rotation (Eq. 4.32),

$$
\left\langle\Gamma_{\mathrm{G}}\right\rangle=-\mathcal{S} \zeta .
$$

Assuming that the laws described by equations (4.36) and (4.38) hold in numerical models of the geodynamo (see section 4.3.3 below for a detailed analysis), the long-term conservation 
of the angular momentum of the mantle and inner core (Eqs. 4.26, 4.27) now become

$$
\begin{aligned}
0 & =\zeta \mathcal{S}+\frac{K_{1}}{E_{\eta}} r_{\mathrm{o}}^{4} B_{\mathrm{r}_{\mathrm{o}}}^{2} \Sigma \mathcal{D}, \text { and } \\
0 & =-\zeta \mathcal{S}+\frac{K_{2}}{E_{\eta}} r_{\mathrm{i}}^{5} B_{\mathrm{r}_{\mathrm{i}}}^{2}\left(\mathcal{C}_{\mathrm{icb}}-\alpha \mathcal{C}_{\mathrm{f}}\right), \quad \text { respectively. }
\end{aligned}
$$

Equation (4.41) can lead to a first statement of the inner core super-rotation. We have

$$
\begin{aligned}
\mathcal{S} & =-\frac{1}{\epsilon_{1}} \mathcal{D}, \quad \text { with } \\
\epsilon_{1} & =\frac{\zeta E_{\eta}}{K_{1} r_{\mathrm{o}}^{4} B_{\mathrm{r}_{\mathrm{o}}}^{2} \Sigma}
\end{aligned}
$$

Another expression of $\mathcal{S}$ can be obtained using the ICB torque balance (4.42):

$$
\begin{aligned}
\mathcal{S} & =\frac{1}{\epsilon_{2}}\left(\mathcal{C}_{\mathrm{icb}}-\alpha \mathcal{C}_{\mathrm{f}}\right), \quad \text { with } \\
\epsilon_{2} & =\frac{\zeta E_{\eta}}{K_{2} r_{\mathrm{i}}^{5} B_{\mathrm{r}_{\mathrm{i}}}^{2}}
\end{aligned}
$$

The meaning of the two parameters $\epsilon_{1}$ and $\epsilon_{2}$ is the following: $\epsilon_{1}$ is the ratio between the gravitational and the electromagnetic coupling strength exerted on the mantle, and $\epsilon_{2}$ describes the same ratio applied to the inner core. We re-express equations (4.43) and (4.45) using the global convective shear $\mathcal{C}_{\mathrm{f}}$ as a control parameter. Then, from the decomposition of equation (4.34) we can express the westward drift and the inner core super-rotation as

$$
\begin{aligned}
\mathcal{D} & =-\frac{\epsilon_{1}(1-\alpha) \mathcal{C}_{\mathrm{f}}}{1+\epsilon_{1}+\epsilon_{2}}, \quad \text { and } \\
\mathcal{S} & =\frac{(1-\alpha) \mathcal{C}_{\mathrm{f}}}{1+\epsilon_{1}+\epsilon_{2}}
\end{aligned}
$$

Finally, we can formulate the link between $\mathcal{S}$ and $\mathcal{D}$ through an equation describing the repartition of the total shear of the system :

$$
\mathcal{S}-\mathcal{D}=(1-\alpha) \mathcal{C}_{\mathrm{f}} \frac{1+\epsilon_{1}}{1+\epsilon_{1}+\epsilon_{2}}
$$

Recall that the validity of this model will be checked against numerical simulations in section 4.3, where we will determine the values of the numerical constants $K_{1}, K_{2}$, and $\alpha$.

\subsubsection{Thermal wind scaling of the convective shear $\mathcal{C}_{\mathrm{f}}$}

Equations (4.47-4.49) suggest that the strengths of both $\mathcal{S}$ and $\mathcal{D}$ are controlled by the state of coupling (i.e $\epsilon_{1}$ and $\epsilon_{2}$ ), and by $\mathcal{C}_{\mathrm{f}}$, the mean shear in the fluid outer core. This shear is a control parameter because it should only depend on the vigor of the convection, 
embodied by the Rayleigh number $\mathcal{R} a_{F}$ (Eq. 4.25). This assumption stems from the thermal wind balance between Coriolis, buoyancy and pressure forces. Considered inside the tangent cylinder, this balance is thought to control the average inner core super-rotation (Aurnou et al., 1996, 1998) and more generally the convective shear $\mathcal{C}_{\mathrm{f}}$. Taking $[\boldsymbol{\nabla} \times(4.5)] \cdot \mathbf{e}_{\varphi}$, the thermal wind steady-state azimuthal velocity obeys

$$
\frac{\partial u_{\varphi}}{\partial z}=\frac{g}{2 r \rho \Omega} \frac{\partial C}{\partial \theta}
$$

in dimensional form. Introducing a typical velocity $U \simeq \mathcal{C}_{\mathrm{f}} D$, a typical advective co-density perturbation can then be evaluated as $C \simeq F / 4 \pi D^{2} U$. Dimensional analysis of equation (4.50) then yields

$$
\mathcal{C}_{\mathrm{f}}^{2} \simeq \frac{1}{2 \theta} \frac{g F}{4 \pi \rho \Omega D^{4}}
$$

Using the tangent cylinder angle $\theta=0.36 \mathrm{rad}$, this gives in dimensionless form

$$
\mathcal{C}_{\mathrm{f}} \simeq 1.2 \mathcal{R} a_{F}^{\frac{1}{2}}
$$

the classical thermal wind scaling (Aurnou et al., 2003; Aubert, 2005), the validity of which will be examined in section 4.3.2.

\subsection{Results}

We analyze now the long-term rotational state of our set of 45 simulations (see tables 4.1 and 4.2), and in particular the influence of the two control parameters $\Sigma$ and $\zeta$. In order to obtain a satisfying parameters survey and end-member cases, we first present the results of numerical simulations with $\Sigma$ varying from $10^{-8}$ to $10^{-1}$, corresponding to a mantle conductance up to one order of magnitude below the core conductance, and simulations with $\zeta$ varying from 0 to 0.75 , this last value being sufficient to lock the whole system by gravitational coupling (Aubert et al., 2013).

\subsubsection{Typical long-term state of differential rotations}

The purpose of this section is to examine the behavior of our system, in terms of the intensity and geometry of both the field and flow. We first focus on four typical cases presented in figure 4.2. Figure 4.2 (top-panel) shows the long-term patterns of the average azimuthal velocities which shear the ambient meridional magnetic field lines to produce the azimuthal field presented in figure 4.2 (bottom-panel). At both the ICB and the CMB, this interaction is the source of electromagnetic torques. The long-term rotational state $\left(\mathcal{D}, \mathcal{S}, \mathcal{C}_{\text {icb }}, \mathcal{C}_{\mathrm{f}}\right)$ corresponding to cases A, B, C and D presented in figure 4.2 is further detailed in figure 4.3. In case A (Fig. 4.2), the gravitational coupling between the inner core and the mantle is set to zero. Thus, the inner core is free to rotate and the inner core 
super-rotation is at its peak (see figure 4.3b). Moreover, the westward drift of fluid close to the CMB is small. For the other cases B, C and D (Fig. 4.2), the gravitational coupling is comparable to core inertia and the inner core rotation is braked such that, on average, $\mathcal{S}$ is almost zero (see figure 4.3a). We can thus focus on the influence of the lower mantle conductivity. Case B has a strong westward drift since the lower mantle conductance is almost insignificant $(\Sigma \ll 1)$. As $\Sigma$ is increased, the westward drift is significantly lowered (case C), down to a point where the fluid close to the CMB is completely locked to the mantle through electromagnetic coupling (case D). The increase of $\Sigma$ also triggers an increase of the shear close to the ICB, mostly occurring in the tangent cylinder. Finally, comparing cases $\mathrm{A}$ and $\mathrm{C}$, we can already envision a link between $\mathcal{S}$ and $\mathcal{D}$. As a matter of fact, for the same lower mantle conductance, a change in the mean differential rotation of the inner core caused by a variation of the gravitational torque also induces a pronounced change in the azimuthal velocity of the fluid close to the CMB. This is achieved while preserving the thermal wind shear between the CMB and the ICB within the tangent cylinder (see shear patterns in figure 4.2, top-panel, and the stability of $\mathcal{C}_{\mathrm{f}}$ in figure 4.3a,b). The systematic impact of the mantle conductance $\Sigma$ and the gravitational coupling strength $\zeta$ is further explored in figure 4.3. Figure 4.3a presents the results of the time-average characteristic rotation rates of our system, in a situation where the inner core and the mantle are strongly gravitationally coupled. In that case, the inner core is indeed completely locked to the mantle, so that $\mathcal{S}$ is vanishingly small. At low values of mantle conductance, the fluid below the $\mathrm{CMB}$ reaches its peak westward rotation rate $(\mathcal{D}$ is maximum), whereas the fluid close to the ICB is rotating eastward $\left(\mathcal{C}_{\text {icb }}>0\right)$. For increasing values of mantle conductance, the strength of the electromagnetic coupling between the mantle and the fluid core increases as well. This results in a decrease of $\mathcal{D}$, as the fluid close to the CMB tends to be more and more locked to the mantle, as observed already in figure 4.2. Remarkably, the shear $\mathcal{C}_{\mathrm{f}}$ in the whole fluid core is confirmed to be roughly constant, over a wide range of mantle conductance. A decreasing $\mathcal{D}$ is thus accompanied by an increasing eastward rotation of the fluid close to the ICB, and therefore a higher local shear $\mathcal{C}_{\text {icb }}$, since the inner core differential rotation rate $\mathcal{S}$ remains close to zero. Figure $4.3 \mathrm{~b}$ displays the effects of the strength of gravitational coupling between the mantle and the inner core, on the rotational dynamics of the system. At low $\zeta$, the inner core is free to rotate. It is therefore entrained in a substantial eastward rotation by the fluid close to the ICB, through electromagnetic coupling. As $\zeta$ is increased, the inner core rotation is progressively braked until a situation of gravitational locking with the mantle, so that $\mathcal{S}$ almost vanishes (Fig. 4.3b). Just as previously (Fig. 4.3a), the shear in the fluid $\mathcal{C}_{\mathrm{f}}$ remains constant. As a consequence, the increase of the shear at the ICB, triggered by the braking of the inner core super-rotation, is balanced by an increase of the westward motion of the fluid close to the CMB. The key observation in figures 4.2 and 4.3 is the stability of the global shear $\mathcal{C}_{\mathrm{f}}$ available in the fluid core. Consequently, any modification of a physical control parameter or directly of fluid flow close to the CMB directly impacts the fluid close to the ICB and the inner core 


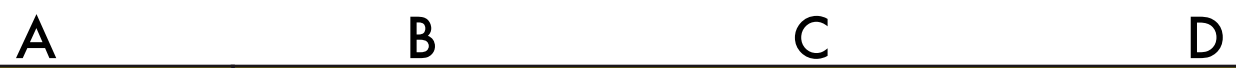

\begin{tabular}{|c|c|c|c|}
\hline $\begin{array}{c}\text { INNER CORE } \\
\text { FREE TO ROTATE }\end{array}$ & \multicolumn{3}{|c|}{$\begin{array}{c}\text { STRONG GRAVITATIONAL COUPLING } \\
\text { INNER CORE LOCKED }\end{array}$} \\
\hline SMALL WD & STRONG WD & WD BRAKING & WD LOCKING \\
\hline$\zeta=0$ & $\zeta=0.75$ & $\zeta=0.75$ & $\zeta=0.75$ \\
$\Sigma=10^{-4}$ & $\Sigma=10^{-8}$ & $\Sigma=10^{-4}$ & $\Sigma=10^{-1}$
\end{tabular}
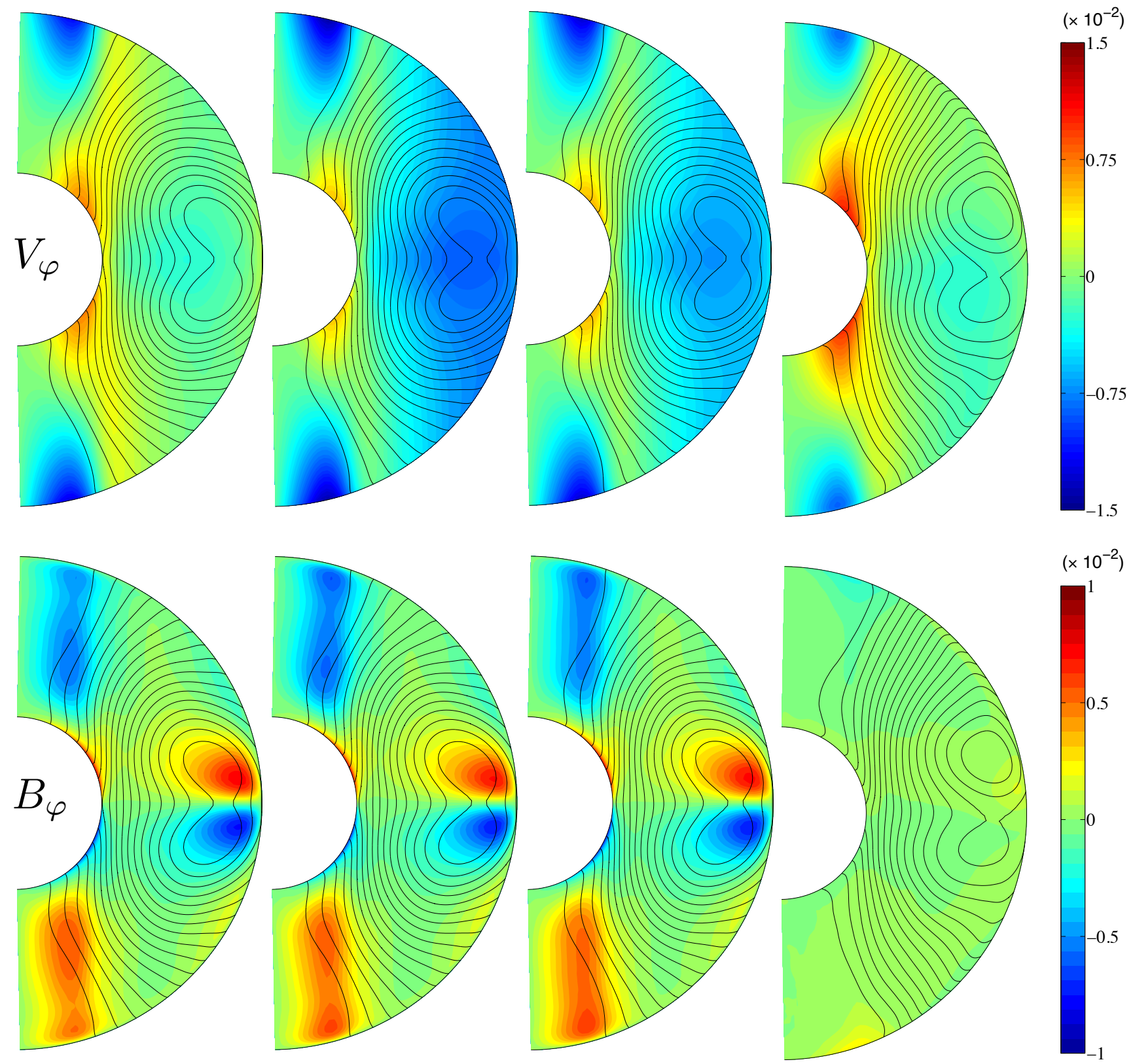

$\left(\times 10^{-2}\right)$

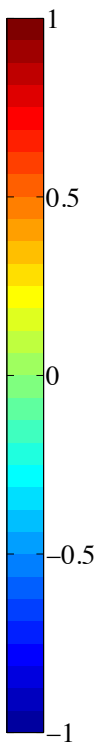

Figure 4.2: Meridional sections of the time and longitude-averaged azimuthal velocity $V_{\varphi}$ (toppanel) and the azimuthal magnetic field $B_{\varphi}$ (bottom-panel) of the numerical simulations highlighted in table 4.1 (rows A, B, C and D). Black lines represent the axisymetric poloidal magnetic field lines. The WD acronym stands for Westward Drift. 

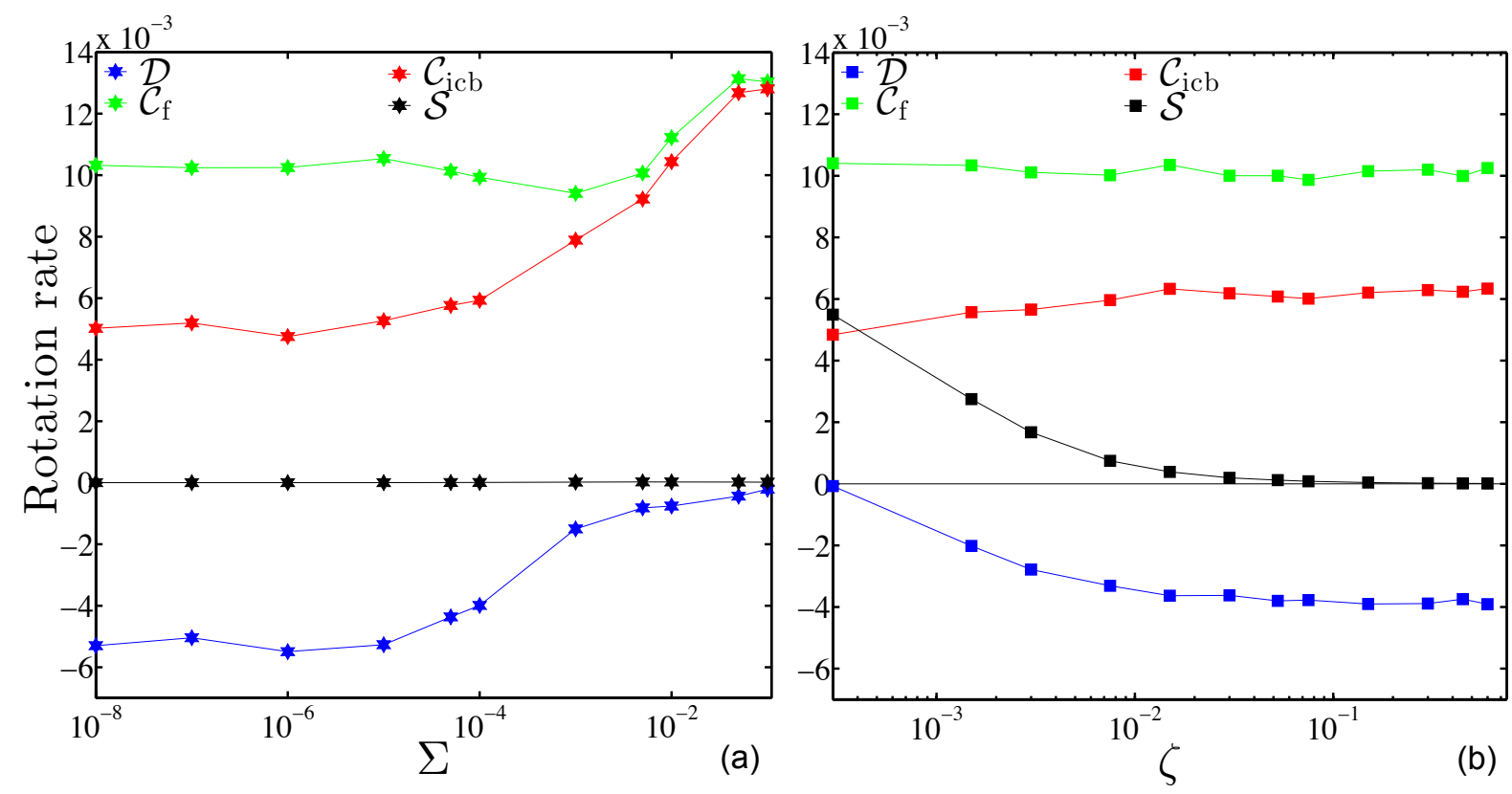

Figure 4.3: Time-average differential rotations $\mathcal{C}_{\text {icb }}, \mathcal{D}, \mathcal{S}$ and $\mathcal{C}_{\mathrm{f}}$ (see Eqs. 4.30, 4.31, 4.32, 4.33) as a function of the mantle conductance $\Sigma$ (with $\zeta=0.75$ ) (a) and the gravitational coupling strength $\zeta$ (with $\Sigma=10^{-4}$ ) (b), in a set of numerical simulations where $\mathcal{R} a_{F}=3 \times 10^{4}, E=3 \times 10^{-5}$ and $E_{\eta}=1.2 \times 10^{-5}$. Labels A,B,C and D refer to simulations of figure 4.2 (see also table 4.1).

axial rotation rate. This observation is thus crucial to formulate the link between $\mathcal{S}$ and $\mathcal{D}$ (Eqs. 4.47-4.49), and their respective dependency on the global convective shear $\mathcal{C}_{\mathrm{f}}$.

\subsubsection{Thermal wind scaling of $\mathcal{C}_{\mathrm{f}}$}

We have seen that $\mathcal{C}_{\mathrm{f}}$ is largely independent on the state of coupling at the boundaries. The leading control of the convection vigor on $\mathcal{C}_{\mathrm{f}}$ (Eq. 4.52 ) is now tested against our numerical dataset in figure 4.4. Equation (4.52) is found to be valid at low values of $\mathcal{R} a_{F}$ with a prefactor rather close to the theoretical value of 1.2 ,

$$
\mathcal{C}_{\mathrm{f}}=2.01 \mathcal{R} a_{F}^{\frac{1}{2}}
$$

The thermal wind scaling is expected to no longer hold at high values of $\mathcal{R} a_{F}$ because inertia starts to disrupt the force balance (see the two rightmost points in figure 4.4). In our numerical simulations, this occurs rather quickly, due to the modest values of the Ekman number, leading to high Rossby numbers, at which our simulations are calculated. Lower Ekman numbers should presumably allow for more inertia before the thermal wind balance is disrupted, thus extending the range of validity of equation (4.52).

\subsubsection{Long-term electromagnetic torques}

We next turn to the analysis of the CMB electromagnetic torque $\left\langle\Gamma_{\mathrm{CMB}}\right\rangle$. In figure 4.5 , we verify first that the linear relationship suggested by equation (4.36) is valid in the range 


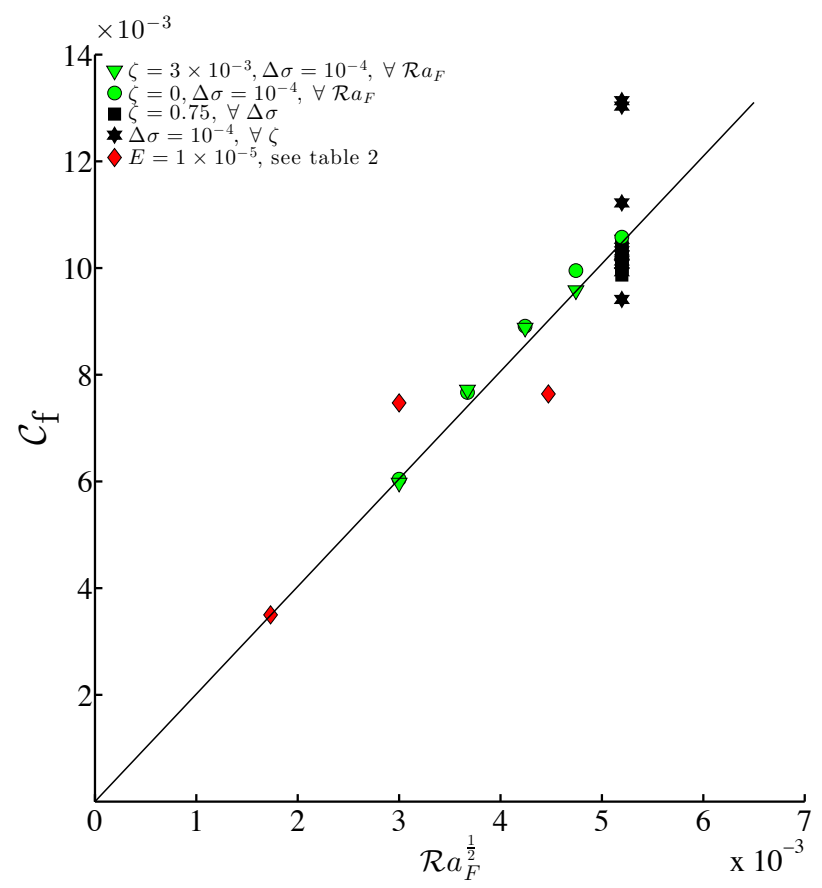

Figure 4.4: Numerical verification of the thermal wind scaling for the available time-average convective shear, $\mathcal{C}_{\mathrm{f}}$ (see Eq. 4.52 and text for details), based on 38 simulations of our suite of models (the remaining 7 are redundant and were used for benchmarking. See appendix 4.5.1).

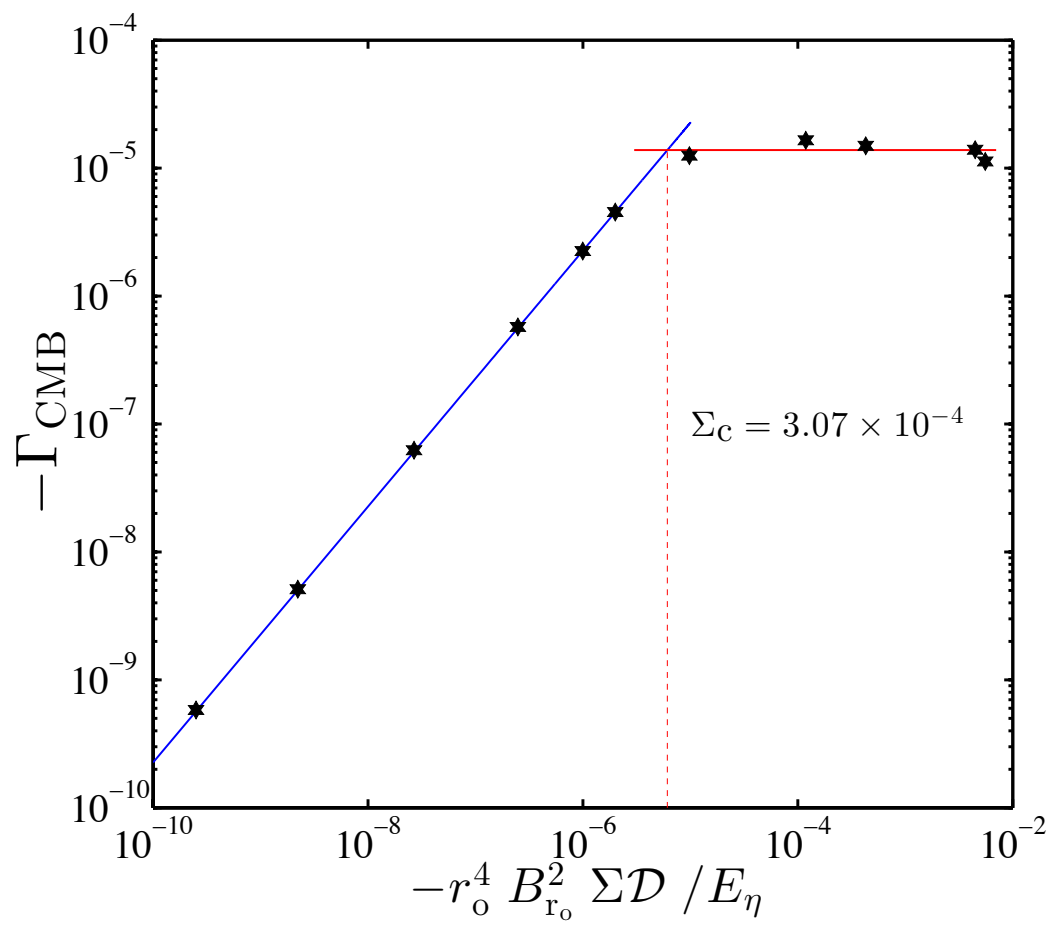

Figure 4.5: Sensitivity of the opposite of the time-average electromagnetic torque of the mantle, $\left\langle\Gamma_{\mathrm{CMB}}\right\rangle$, with respect to its control parameters $r_{\mathrm{o}}, B_{\mathrm{r}_{\mathrm{o}}}^{2}, \Sigma$ and $\mathcal{D}$. Same set of numerical simulations as figure 4.3a. The blue line represents a linear trend (unit slope) 


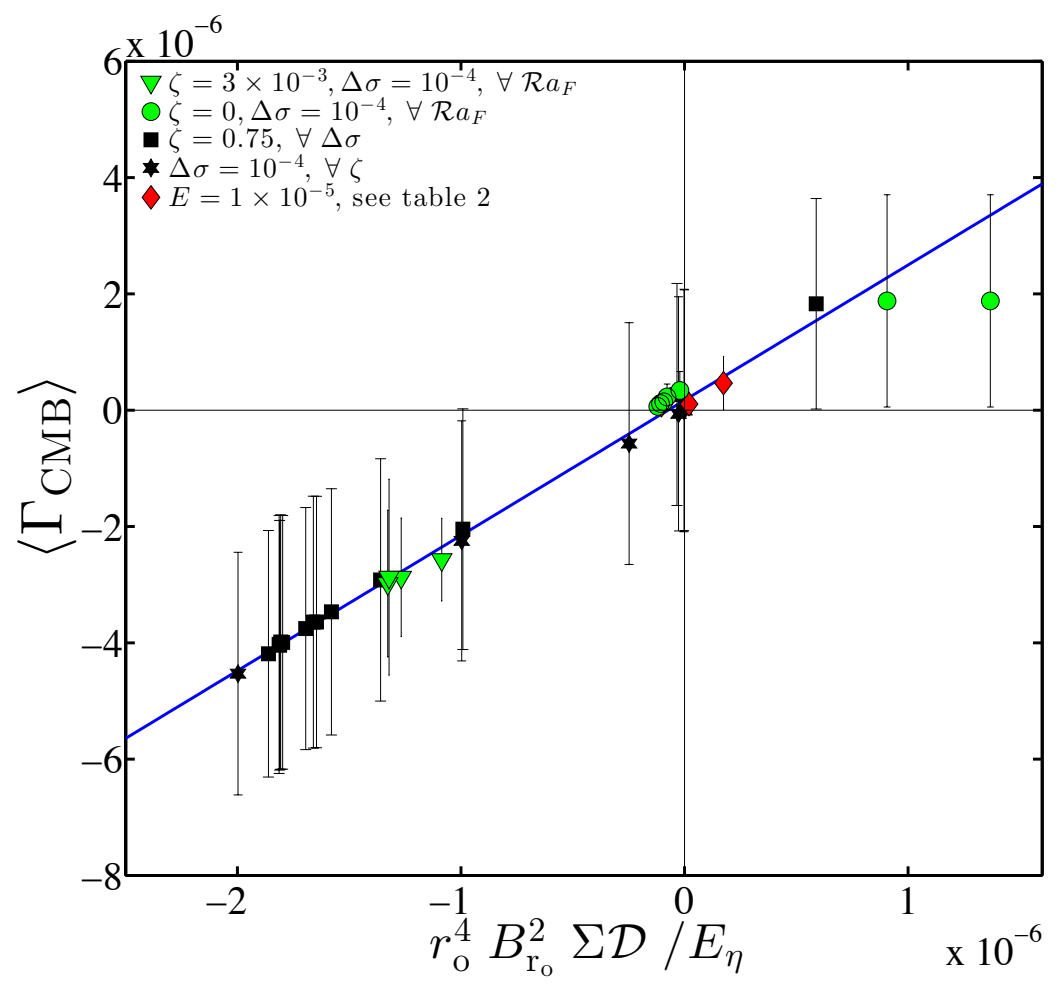

Figure 4.6: Numerical verification of the scaling law for the time average electromagnetic torque at the CMB, $\left\langle\Gamma_{\mathrm{CMB}}\right\rangle$, (see Eq. 4.36 and text for details) for the 33 simulations where $\Sigma<\Sigma_{\mathrm{c}}$. Uncertainties as defined in Appendix 4.5.1.

$0<\Sigma<3.07 \times 10^{-4}$, with $K_{1}=2.3$, determined by least-squares regression. For values of $\Sigma$ beyond $\Sigma_{\mathrm{c}}$, we observe a saturation of the magnetic torque. This is due to the fact that this torque, as defined by equation (4.36), is necessarily limited in a self-sustained, power-limited dynamo where the magnetic field strength itself is bounded. For $\Sigma>\Sigma_{\mathrm{c}}$ also, the saturation of the CMB magnetic torque then implies a decreasing amplitude for the westward drift $\mathcal{D}$. From here, we thus exclude these numerical simulations for which $\Sigma>\Sigma_{\mathrm{c}}$, as they also reflect unrealistic geophysical situations (see section 4.4). Equation (4.36) is then further validated in figure 4.6, where we present the evolution of the magnetic torque exerted on the mantle for a larger subset of numerical runs verifying $\Sigma<\Sigma_{\mathrm{c}}$. Based on the study of Holme (1998), the value of $K_{1}$ estimated by Dumberry (2007) was 1.3. Though our value of 2.3 is a bit higher, it is still of order one thus validating the theoretical model of Dumberry (2007). For the electromagnetic torque at the ICB, $\left\langle\Gamma_{\text {ICB }}\right\rangle$, we seek to prove the consistency of equation (4.38). This scaling law involves the parameter $\alpha$, representing the ratio between the local and remote contributions of the fluid on $\left\langle\Gamma_{\text {ICB }}\right\rangle$. This parameter can be evaluated in numerical simulations where $\left\langle\Gamma_{\mathrm{ICB}}\right\rangle$ must vanish on average, i.e in simulations without gravitational coupling between the inner core and the mantle, $\left\langle\Gamma_{\mathrm{G}}\right\rangle=0$. In figure 4.7 we find that $\alpha$ is roughly constant and evolves between 0.32 and 0.55 , with an average at 0.4 . This is slightly higher than the value 0.22 obtained by Dumberry (2007), in an idealized configuration. Finally, in a situation where gravitational coupling is present, we have seen (Fig. 4.3b) that $\mathcal{C}_{\text {icb }}$ increases while $\alpha$ and $\mathcal{C}_{\text {f }}$ remain 


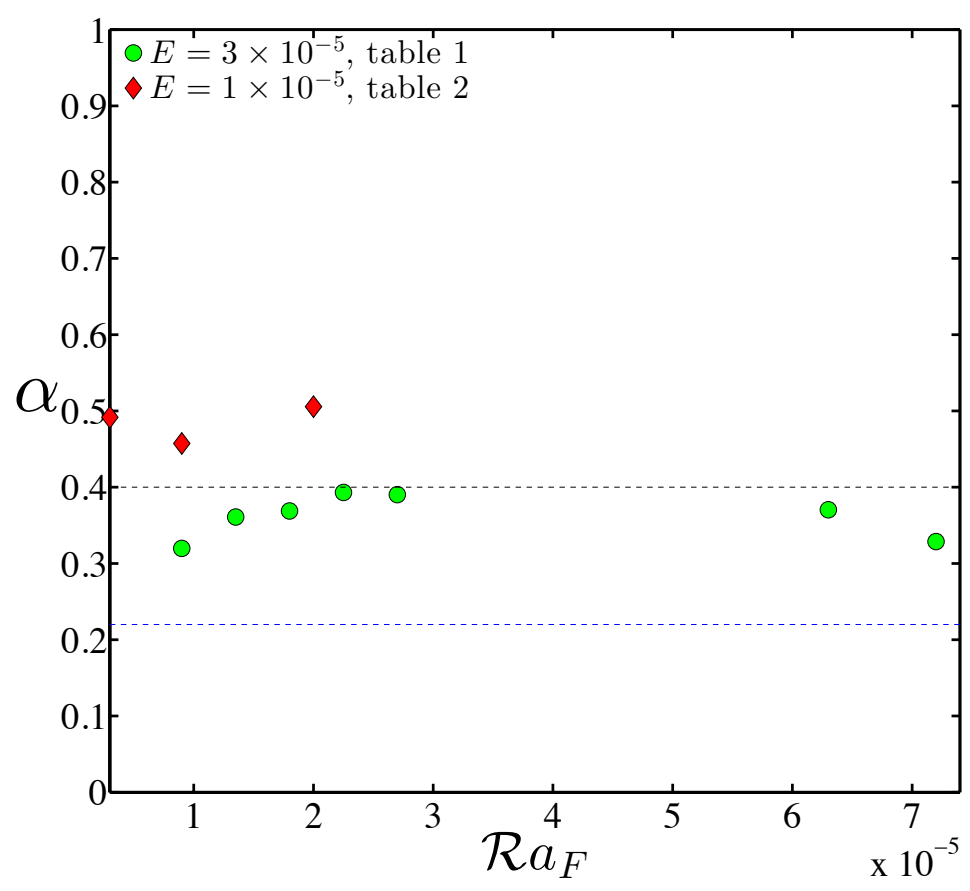

Figure 4.7: Parameter dependence of the ratio $\alpha$ between local and remote shear influencing the time average electromagnetic torque at the ICB, $\left\langle\Gamma_{\mathrm{ICB}}\right\rangle$, as defined in equation (4.38), in the 10 simulations without gravitational coupling (see tables 4.1 and 4.2). The blue dashed-line marks the value obtained by Dumberry (2007), the black line represents the mean of our 10 numerical estimates.

stable. This should produce a linear trend in $\left\langle\Gamma_{\mathrm{ICB}}\right\rangle$, which we indeed observe in figure 4.8. Using a least-squares fitting, we obtain $K_{2}=1.1 \times 10^{-3}$, much smaller than the $\mathcal{O}(1)$ value of Dumberry (2007). We notice here the difference in behavior between a self-sustained dynamo minimizing the magnetic interactions between field and flow (Aubert, 2005), and a forced system where these interactions are maximum. This difference is best seen if we compare the poloidal magnetic field lines of an idealized model (figures A1 and A2 in Dumberry (2007)) with those in the tangent cylinder in figure 4.2. It is obvious that in the latter case, the field lines have adjusted to the azimuthal flow contours in order to minimize the interaction according to the Ferraro effect (Ferraro, 1937) while they are configured for a maximum interaction in the former case. This feedback, leading to a small value of $K_{2}$, could not exist in the framework of Dumberry (2007) and explains the quantitative differences in our results.

\subsubsection{Link between $\mathcal{S}$ and $\mathcal{D}$}

Figure 4.9 shows the agreement of our theoretical laws (4.47) and (4.48) with numerical simulations. The contours of the predicted westward drift and inner core super-rotation are represented as functions of $\epsilon_{1}$ (Eq. 4.44) and $\epsilon_{2}$ (Eq. 4.46). We show here that the amount of shear in the fluid core which is effectively shared between $\mathcal{D}$ and $\mathcal{S}$ is $(1-\alpha) \mathcal{C}_{\mathrm{f}}$. The arrows on the bottom left corners of figures $4.9 \mathrm{a}$ and $4.9 \mathrm{~b}$ point towards the numerical 


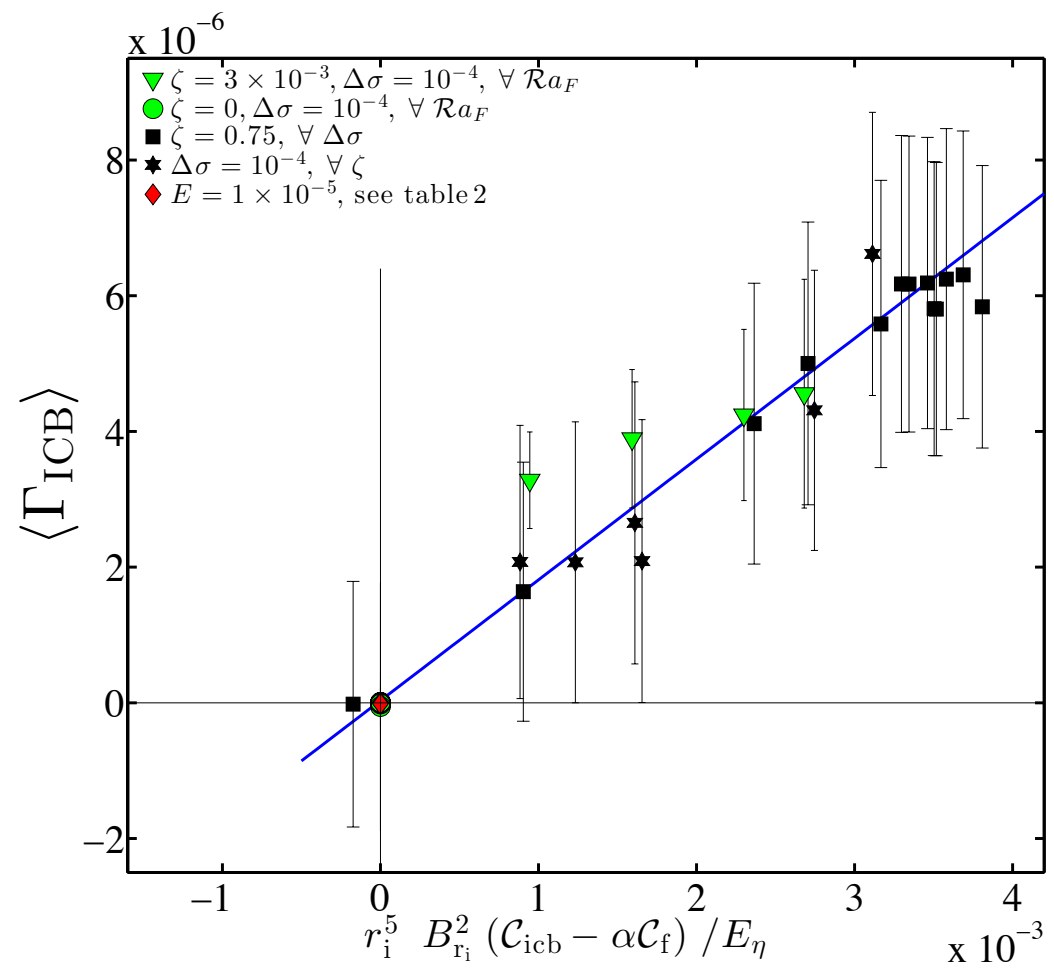

Figure 4.8: Numerical verification of the scaling law for the time average electromagnetic torque at the ICB, $\left\langle\Gamma_{\text {ICB }}\right\rangle$, (see Eq. 4.38 and text for details) for the 33 simulations where $\Sigma<\Sigma_{\mathrm{c}}$. Uncertainties as defined in Appendix 4.5.1.

simulations with no gravitational coupling $(\zeta=0)$, such that $\epsilon_{1} \mapsto 0$ and $\epsilon_{2} \mapsto 0$. In this situation, the whole effective shear available is located at the ICB, so that $\mathcal{S}$ is at its peak and $\mathcal{D}$ equals zero. For strong values of both the gravitational coupling and mantle conductance, $\epsilon_{1} \mapsto 0$ and $\epsilon_{2} \mapsto \infty$, bottom right corners, the shear is located at the ICB, but the inner core is locked to the mantle. In that case, both $\mathcal{S}$ and $\mathcal{D}$ vanish. In order to obtain a strong westward drift, the inner core must be gravitationally braked and the mantle conductance must remain bounded (top left corners Fig.4.9a,b). Finally, these figures underline two major conclusions on the behavior of the rotational dynamics of the set of coupled Earth dynamos envisaged for this study. First, as the effective shear in the fluid core is a constant, there is a clear link between the geomagnetic westward drift and the inner core super-rotation. Second, the transition between the regimes of strong $\mathcal{S} /$ vanishing $\mathcal{D}$ and strong $\mathcal{D}$ / vanishing $\mathcal{S}$ appears to be rather sharp, as it occurs over two orders of magnitude of the control parameters $\epsilon_{1}$ and $\epsilon_{2}$. As a consequence, a reasonable assumption is to consider that the available shear in the fluid core of the Earth is either in the inner core super-rotation or in the westward drift, but not distributed among the two. This also suggests that estimates of $\mathcal{D}$ and $\mathcal{S}$ for the Earth are likely to place tight constraints on the values of $\epsilon_{1}$ and $\epsilon_{2}$, and consequently on the geophysical parameters entering their definition (see the discussion below). 

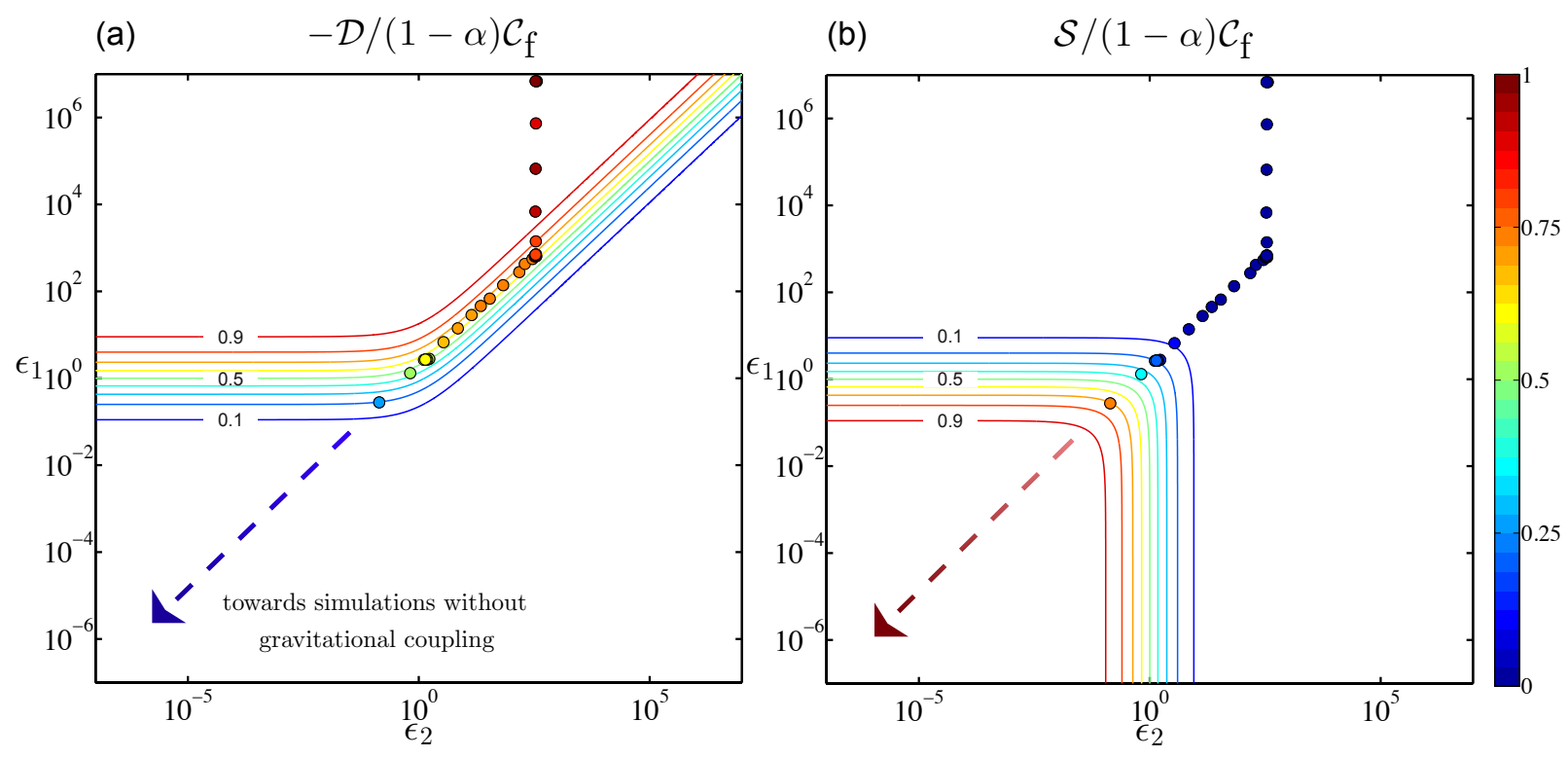

Figure 4.9: Contours of the predicted westward drift $\mathcal{D}($ a) and inner core super-rotation $\mathcal{S}$ (b) determined from equations (4.47) and (4.48). Comparison with the magnitude of $\mathcal{D}$ and $\mathcal{S}$ (colored circles) in our 27 numerical simulations with $\Sigma<\Sigma_{\mathrm{c}}$ and $\mathcal{R} a_{F}=2.7 \times 10^{-4}$ (see tables 4.1 and 4.2). Arrows point towards simulations with no gravitational coupling, i.e $\zeta=0$.

\subsection{Discussion}

Our suite of numerical simulations stresses the tight link between the inner core superrotation $\mathcal{S}$ and the geomagnetic westward drift $\mathcal{D}$, as components of the long-term rotational dynamics in the Earth's core. While we rely on the theoretical approach of Dumberry (2007) to understand our results, in particular in order to analyze the long-term electromagnetic torques at work at the $\mathrm{CMB}$ and at the ICB, it is noteworthy that the extensive numerical study we carried out sheds new light on several aspects of the long-term rotational dynamics of the fluid outer core. First, we determined the phase diagrams for $\mathcal{S}$ and $\mathcal{D}$ (Fig. 4.9), together with their dependency on the convective forcing (Eq. 4.49), through the convective shear $\mathcal{C}_{\mathrm{f}}$ which is distributed among these two quantities. In contrast, the geomagnetic westward drift is considered as a given input in the study of Dumberry (2007). Second, the fact that the models (Eqs. 4.36, 4.38) provide a satisfactory description of the electromagnetic torques at the fluid outer core boundaries, while only involving spherical rotation rates, needed a validation that we provide here. Finally, the very observation of the Ferraro effect diminishing the value of $K_{2}$ in equation (4.38) arises from a validation in self-consistent numerical simulations of the geodynamo, and could not be expected in Dumberry (2007). All these elements now give us confidence in applying our results to the Earth's core. The first quantity we wish to estimate is the convective shear $\mathcal{C}_{\mathrm{f}}$ given by equation (4.53), which we now present in the dimensional form,

$$
\mathcal{C}_{\mathrm{f}}=2.01 \Omega \mathcal{R} a_{F}^{\frac{1}{2}} .
$$


The flux Rayleigh number $\mathcal{R} a_{F}$ defined in equation 4.25 may also be expressed as a function of the convective dynamo power (Aubert et al., 2009) through

$$
R a_{F}=\frac{1}{\gamma} \frac{p}{\rho \Omega^{3} D^{2} V}
$$

Here $V$ is the core volume and $\gamma$ the conversion fraction (Eq. 18 in Aubert et al., 2009) between power $p$ and mass anomaly flux $F$. We use $\rho=11000 \mathrm{~kg} . \mathrm{m}^{-3}$ and $\Omega=7.29 \times$ $10^{-5}$ rad.s ${ }^{-1}$ and envision a situation where the geodynamo is entirely chemically-driven, meaning a bottom-driven convection with $\gamma=0.33$ (Aubert et al., 2009). The heat flux at the core mantle boundary $Q_{\mathrm{CMB}}$ is assumed to match the adiabatic value of $15 \mathrm{TW}$ (Pozzo et al., 2012), so that the dynamo power is then $p=\epsilon Q_{\mathrm{CMB}}$ with $\epsilon=0.2$ being the combined efficiency of chemical convection and latent heat release at the ICB. This finally yields $\mathcal{R} a_{F}=2.5 \times 10^{-12}$ and thus $\mathcal{C}_{\mathrm{f}}=0.42^{\circ} \mathrm{yr}^{-1}$. Using our mean value of $\alpha=0.4$ (Fig. 4.7), we show here that the available shear distributed into $\mathcal{S}$ and $\mathcal{D}$ is then $(1-\alpha) \mathcal{C}_{\mathrm{f}}=0.25^{\circ} \mathrm{yr}^{-1}$ Remarkably, this is close to the mean value of the geomagnetic westward drift of $0.28^{\circ} \mathrm{yr}^{-1}$ in the Atlantic hemisphere over the last 400 years estimated by Finlay and Jackson (2003). This strongly suggests that a significant part, if not the whole shear available is currently in the geomagnetic westward drift, leaving the long-term inner core super-rotation close to 0 , as inferred by seismological studies (see e.g. Souriau and Calvet, 2015, for a recent review of these). In order to have a second estimate of the proportion of the convective shear available for $\mathcal{S}$ or $\mathcal{D}$, we need to assess the values of $\epsilon_{1}$ and $\epsilon_{2}$ for the Earth. The dimensional form of $\epsilon_{1}$ and $\epsilon_{2}$ provides an overview of the geophysical parameters we have to take into account,

$$
\begin{aligned}
\epsilon_{1} & =\frac{\Gamma \tau}{K_{1} B_{\mathrm{r}_{\mathrm{o}}}^{2} \Delta \sigma_{\mathrm{m}} r_{\mathrm{o}}^{4}} \\
\epsilon_{2} & =\frac{\Gamma \tau}{K_{2} B_{\mathrm{r}_{\mathrm{i}}}^{2} \sigma_{\mathrm{c}} r_{\mathrm{i}}^{5}}
\end{aligned}
$$

In our estimates, we use $r_{\mathrm{i}}=1220 \mathrm{~km}, r_{\mathrm{o}}=3480 \mathrm{~km}, B_{\mathrm{r}_{\mathrm{o}}}=0.4 \mathrm{mT}$, and the values of $K_{1}$ and $K_{2}$ found in section $4.3 .3\left(K_{1}=2.3, K_{2}=1.1 \times 10^{-3}\right)$. Our simulations (Tables 4.1 and 4.2) for geophysically realistic values of the mantle conductance consistently exhibit a ratio $B_{\mathrm{r}_{\mathrm{i}}} / B_{\mathrm{r}_{\mathrm{o}}} \sim 7$; so we thus set the r.m.s magnetic field at the inner core boundary to $B_{\mathrm{r}_{\mathrm{i}}}=2.8 \mathrm{mT}$, in agreement with inferences of the magnetic field strength inside the core (Gillet et al., 2010). We also adopt the range $3 \times 10^{19} \mathrm{~N} . \mathrm{m}<\Gamma<2 \times 10^{20} \mathrm{~N} . \mathrm{m}$ proposed by Davies et al. (2014) for the parameter $\Gamma$, relative to the mantle heterogeneities at the source of the gravitational torque. The core conductivity $\sigma_{\mathrm{c}}$ is set to $1.5 \times 10^{6}{\mathrm{~S} . \mathrm{m}^{-1}}^{-1}$ according to Pozzo et al. (2012). The major uncertainties to assess $\epsilon_{1}$ and $\epsilon_{2}$ then lie in the lower mantle conductance, $\Delta \sigma_{\mathrm{m}}$, and the viscous deformation time of the inner core, $\tau$. Based on the observed out-of-phase component of the forced nutations of the Earth, Buffett (1992) inferred a lower mantle conductance of $10^{8} \mathrm{~S}$. This value is often considered 
as a minimum to ensure a sufficiently strong direct electromagnetic coupling to couple the core and the mantle. Buffett (1992) proposed the existence of a thin layer at the base of the mantle, about 200 meters thick, with the same conductivity as that of the core. From a mineral physics point a view, this high conductive layer may be composed of FeO, whose conductivity was estimated as close to $\sigma_{\mathrm{c}}$ by Ohta et al. (2012). Another way to obtain a reasonably conducting lower mantle is to consider a thicker layer $(200-300 \mathrm{~km})$ of $(\mathrm{Mg}, \mathrm{Fe}) \mathrm{SiO}_{3}$ post-perovskite, which may have an electrical conductivity greater than $10^{2} \mathrm{~S}^{-\mathrm{m}^{-1}}$ (Ohta et al., 2008). This would lead to a conductance larger than $2 \times 10^{7} \mathrm{~S}$. The mantle conductance is also bound on the upper side by the observation of high frequencies in the core magnetic signal, constraining $\Delta \sigma_{\mathrm{m}}$ to be lower than $2.5 \times 10^{9} \mathrm{~S}$ (Dumberry, 2007). We thus adopt a range of $2 \times 10^{7} \mathrm{~S}<\Delta \sigma_{\mathrm{m}}<2.5 \times 10^{9} \mathrm{~S}$. Note that this range mostly lies below the limit value $\Sigma_{\mathrm{c}} \sigma_{\mathrm{c}} D=10^{9} \mathrm{~S}$ previously introduced in figure 4.5, meaning that our scaling laws are valid. Finally, we constrain $\tau$ using the recent mineral physics experiments of Gleason and Mao (2013) who reported an inner core viscosity range of $10^{15}-10^{18}$ Pa.s, corresponding to $\tau=0.02 \mathrm{yr}$ and $\tau=20 \mathrm{yr}$ (Buffett, 1997). Considering all uncertainties on the geophysical parameters mentioned above, we obtain the following ranges of foreseeable values for $\epsilon_{1}$ and $\epsilon_{2}$,

$$
\begin{aligned}
& 1.4 \times 10^{-4} \leq \epsilon_{1} \leq 1.1 \times 10^{2} \\
& 5.4 \times 10^{-4} \leq \epsilon_{2} \leq 3.6
\end{aligned}
$$

This indicates that our current knowledge of deep Earth physical parameters does not strongly constrain the partitioning of $(1-\alpha) \mathcal{C}_{\mathrm{f}}$ into $\mathcal{S}$ and $\mathcal{D}$. However, for $\Delta \sigma_{\mathrm{c}}$ of order $10^{8} \mathrm{~S}$, we may re-express our results in order to formulate a condition on the inner core viscous relaxation time for dominant westward drift. With the previously used value of the other geophysical parameters this gives $\epsilon_{1} \approx \epsilon_{2}$, and the condition for a dominant westward drift according to figure 4.9 is then $\epsilon_{1}>1$ or $\epsilon_{2}>1$. This in turn yields $\tau>4 \mathrm{yr}$, meaning that the inner core must be moderately stiff, having a viscosity larger than $2 \times 10^{17} \mathrm{~Pa}$.s. Note finally that in the case $\epsilon_{1} \approx \epsilon_{2}$, the magnitude of the shear that can be distributed into $\mathcal{S}$ and $\mathcal{D}$ is $(1-\alpha) \mathcal{C}_{\mathrm{f}} \frac{1+\epsilon_{1}}{1+2 \epsilon_{1}}$, meaning that only $(1-\alpha) \mathcal{C}_{\mathrm{f}} / 2=0.125^{\circ} \mathrm{yr}^{-1}$ is available if $\tau>4 \mathrm{yr}$ (with $\epsilon_{1}=\epsilon_{2} \gg \mathcal{O}(1)$ ). The drift so available is axisymmetric. In order to match the westward drift of $0.28^{\circ} \mathrm{yr}^{-1}$ at low latitude in the Atlantic hemisphere estimated by Finlay and Jackson (2003) from historical records, an additional mechanism has to be invoked that can increase the drift rate in the Atlantic hemisphere at the expense of its Pacific counterpart. A geophysically sound possibility is that of heterogeneous buoyancy fluxes at the CMB and ICB, as advocated by Aubert et al. (2013). A hemispherical differential buoyancy release of spherical harmonic degree 1 and order 1 at the ICB can indeed generate a concentration of the drift in the Atlantic hemisphere for several centuries, leading to drift rates of about $0.23^{\circ} \mathrm{yr}^{-1}$ in this region, and a very weak drift in the Pacific hemisphere. An alternative to this mechanism is that of slow magnetic waves riding on 


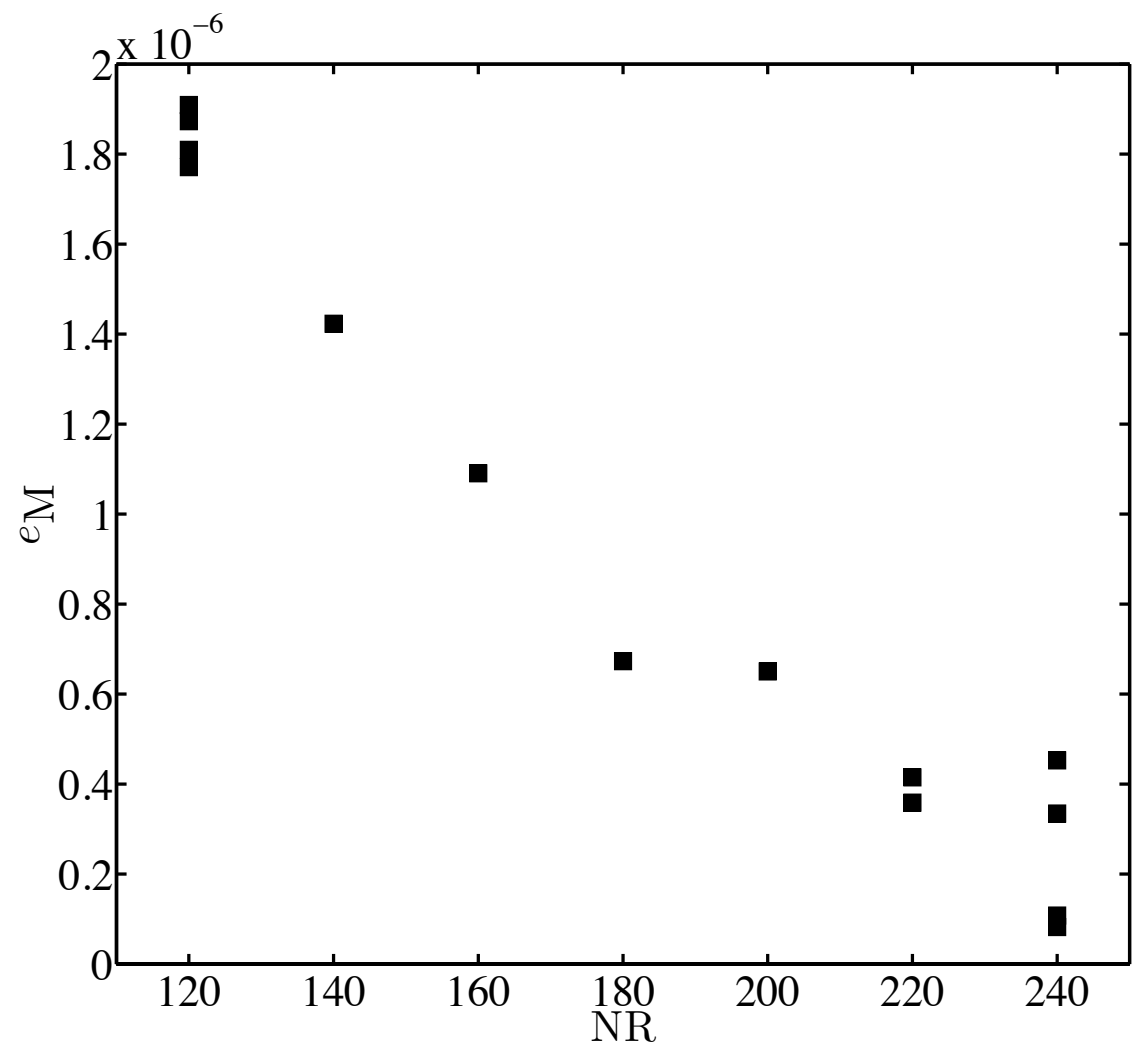

Figure 4.10: Total torque balance $e_{\mathrm{M}}$ of statistically converged simulations as a function of the number of radial grid points $N R$ in the fluid shell.

top of the mean westward flow (see e.g. Hori et al., 2015). For the sake of consistency, the waves so envisioned should then be able to account for the hemispherical dichotomy of the westward drift deduced from historical records, while yielding drift rates of the order of $0.1^{\circ} \mathrm{yr}^{-1}$ to $0.2^{\circ} \mathrm{yr}^{-1}$ near the equator.

\subsection{Appendix}

\subsubsection{Assessment of numerical uncertainties on torques}

Each error bar pictured in figures 4.6 and 4.8 reflects the truncation error due to the numerical approximation of the model. In order to make our systematic analysis numerically tractable, we indeed had to use a moderate resolution (Tables 4.1 and 4.2). This error manifests itself in the long-term magnitudes of the torques. An estimate of the magnitude of this error is provided by the quantity

$$
e_{\mathrm{M}}=\left\|\left\langle\Gamma_{\mathrm{CMB}}>+<\Gamma_{\mathrm{ICB}}\right\rangle\right\|
$$

The error $e_{M}$ should ideally vanish (Eq. 4.13) and indeed converges towards 0 at a rate consistent with a second-order finite difference scheme in radius, when the radial resolution $N R$ is increased (see figure 4.10). 


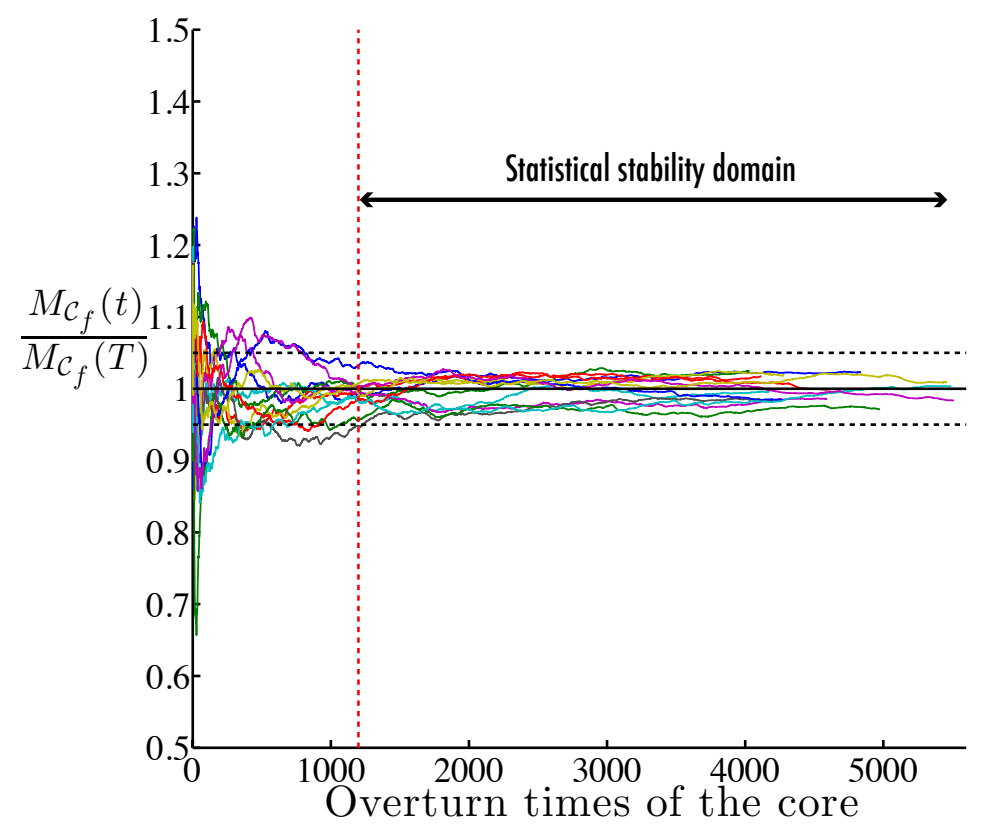

Figure 4.11: Evolution of the cumulative mean of $\mathcal{C}_{\mathrm{f}}$ over time in simulations with varying $\zeta$ and $\Sigma=10^{-4}$.

\subsubsection{Statistical convergence}

Since our simulations are highly time-dependent, we analyze the cumulative mean $M$ of each datum $x$ over time. The time-dependent cumulative mean is denoted by $M x(t)$. We consider a simulation as statistically converged if the following criterion is met. At each point in time $t \leq T$ (the total time of integration), $M x(t)$ is within $5 \%$ of $M x(T)$. As an example, figure 4.11 shows the statistical convergence of $\mathcal{C}_{\mathrm{f}}$ in those 13 simulations that were used to produce figure $4.3 \mathrm{~b}$ (constant $\Sigma$, varying $\zeta$ ). We observe in this case that it takes on the order of 1000 turnover times to achieve statistical convergence for $\mathcal{C}_{\mathrm{f}}$. The same order of magnitude of 1000 turnover times applies to other diagnostics.

\section{Acknowledgements}

The authors would like to thank Mathieu Dumberry for discussions and comments on an early draft of the manuscript. We also thank two anonymous referees for their constructive and detailed reviews which greatly helped to improve the manuscript. JA and AF were supported by the French "Agence Nationale de la Recherche" under the grant ANR-2011-BS56-011. JA also acknowledges support from French "Programme National de Planétologie" (PNP, INSU/CNRS) in 2014. Numerical computations were performed at SCAPAD, IPGP, France and using HPC resources from GENCI-IDRIS (Grants 2014-042122 and 2015-042122). This is IPGP contribution 3692. 


\title{
Time-dependent electromagnetic torques
}

\begin{abstract}
This chapter is dedicated to the formulation of time-dependent electromagnetic torque models at the fluid core boundaries. To this end, section 5.1 is dedicated to solutions describing the diffusion of a time-dependent perturbation in the azimuthal magnetic field into the inner core, the fluid outer core and the electrically conducting part of the mantle. These solutions allow the expression of the time-dependent azimuthal field at the fluid core boundaries, developed in section 5.2, through the treatment of the conservation of the tangential electric field at the inner core boundary (ICB) and the core-mantle boundary (CMB). This work is based on a demonstration developed by Buffett (1992) and establishes the expressions of two transfer functions between the velocity jumps at both ICB and CMB and the perturbations of the magnetic field that they produce to finally enter the models of the time-dependent electromagnetic torques in section 5.3.
\end{abstract}

\section{Résumé}

Ce chapitre est consacré à la formulation de modèles de couple électromagnétique dépendant du temps aux limites du noyau fluide. A cet effet, la section 5.1 est dédiée aux solutions décrivant la diffusion d'une perturbation dépendante du temps du champ magnétique azimutal dans la graine, le noyau fluide et la partie conductrice du manteau. Ces solutions permettent l'expression du champ azimutal aux limites du noyau fluide (section 5.2) à travers le traitement de la conservation du champ électrique tangentiel à l'ICB et à la CMB. Ce travail est basé sur une démonstration développée par Buffett (1992) et établit les expressions de deux fonctions de transfert entre les sauts de vitesse et les perturbations du champ magnétique qu'ils produisent aux frontières du noyau fluide. Ces relations permettent alors de formuler des modèles de couple électromagnétique dépendants du temps dans la section 5.3. 


\subsection{Diffusion of a perturbation of the magnetic field}

We next turn to the analysis of the time-dependent behavior of our coupled core-mantleinner core system. The first step toward the derivation of a time-dependent relationship between the inner core differential rotation and the geomagnetic westward drift is to build dynamical expressions of the electromagnetic torques at the fluid core boundaries. In the long-term, we recall that these expressions are obtained by considering the balance between the advection of the radial magnetic field $B_{\mathrm{r}}$ by the azimuthal velocity $u_{\varphi}$ and the diffusion of the azimuthal field $B_{\varphi}$ produced into the inner core or a thin electrically conducting layer at the base of the mantle. The expression of $B_{\varphi}$ obtained is then used to approximate the electromagnetic torque exerted on the inner core

$$
\Gamma_{\mathrm{ICB}}=\frac{r_{\mathrm{i}}}{\mu_{0}} \int_{S_{\mathrm{ICB}}} B_{\mathrm{r}} B_{\varphi} \sin \theta \mathrm{dS}
$$

with $r_{\mathrm{i}}$ the radius and $S_{\mathrm{ICB}}$ the surface of the inner core, and the electromagnetic torque on the mantle

$$
\Gamma_{\mathrm{CMB}}=-\frac{r_{\mathrm{o}}}{\mu_{0}} \int_{S_{\mathrm{CMB}}} B_{\mathrm{r}} B_{\varphi} \sin \theta \mathrm{dS} .
$$

with $r_{\mathrm{o}}$ the radius of the core and $S_{\mathrm{CMB}}$ the surface of the core-mantle boundary. In a timedependent analysis, this long-term balance is disrupted by the presence of the time-varying term $\partial \mathbf{B} / \partial t$; therefore the azimuthal magnetic field $B_{\varphi}$ that enters the electromagnetic torque integrals can no longer be easily approximated, and requires a specific treatment.

\subsubsection{General arguments}

The demonstration developed below is based on a perturbation analysis where the magnetic field $\mathbf{B}$ and the velocity field $\mathbf{u}$ are both decomposed into their main and perturbed components according to

$$
\begin{aligned}
& \mathbf{B}=\mathbf{B}_{0}+\mathbf{B}_{1}, \quad \text { and } \\
& \mathbf{u}=\mathbf{u}_{0}+\mathbf{u}_{1} .
\end{aligned}
$$

The main magnetic field $\mathbf{B}_{0}$, with spherical coordinate components $\left(B_{\mathrm{r}}^{0}, B_{\theta}^{0}, B_{\varphi}^{0}\right)$, and velocity field $\mathbf{u}_{0}\left(u_{r}^{0}, u_{\theta}^{0}, u_{\varphi}^{0}\right)$ are varying slowly over time and assumed as large scale, that is to say with spacial variations of the order of the fluid outer core thickness $D$. Conversely, the perturbations $\mathbf{B}_{1}\left(B_{\mathrm{r}}, B_{\theta}, B_{\varphi}\right)$ and $\mathbf{u}_{1}\left(0,0, u_{\varphi}\right)$, are smaller in magnitude, varying over time on smaller spatial scales, that will be the subject of a following discussion. We thus have

$$
\begin{aligned}
\left\|\mathbf{B}_{0}\right\| & \gg\left\|\mathbf{B}_{1}\right\|, \quad \text { and } \\
\left\|\mathbf{u}_{0}\right\| & \gg\left\|\mathbf{u}_{1}\right\|,
\end{aligned}
$$




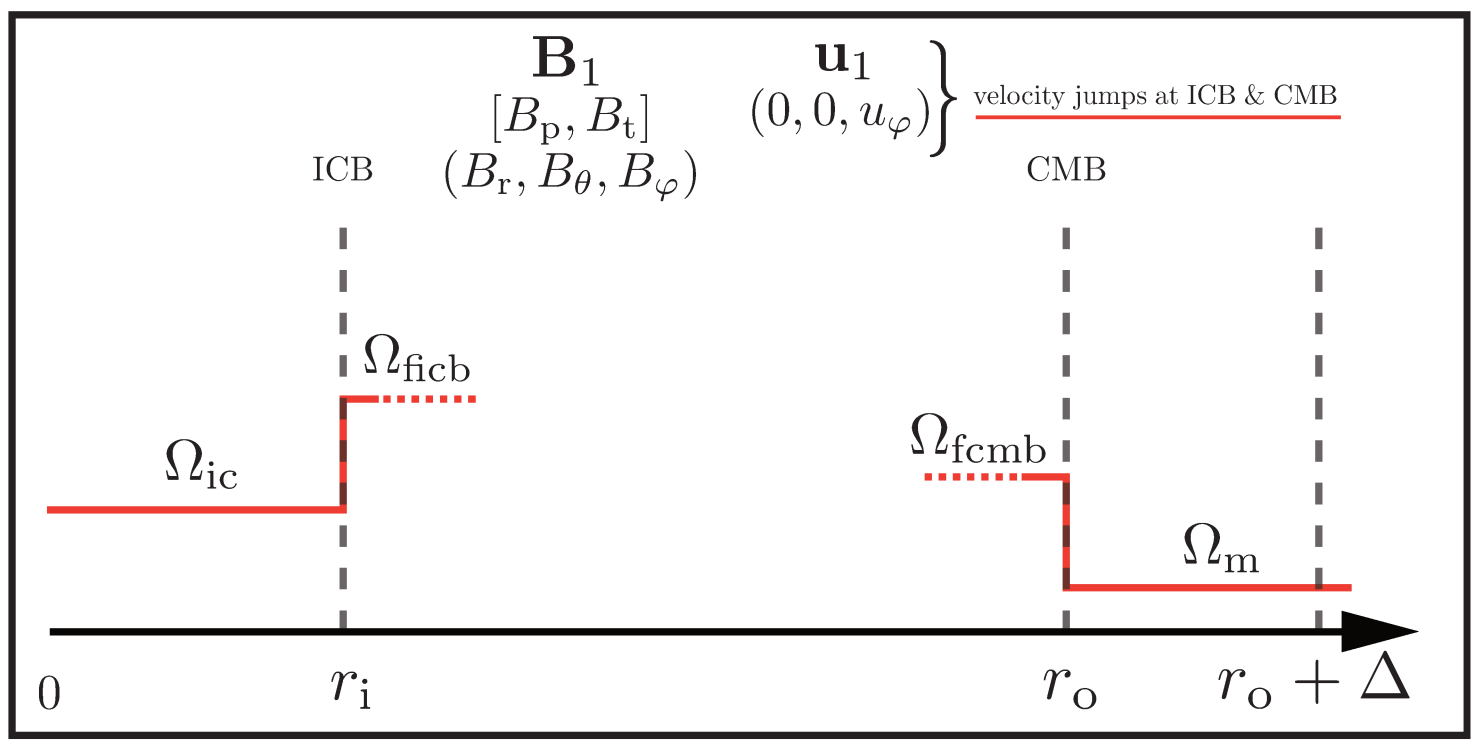

Figure 5.1: Schematic of the system studied in this chapter. The velocity jumps of the perturbed velocity field $\mathbf{u}_{1}\left(0,0, u_{\varphi}\right)$, in red, at both ICB and CMB give rise to a perturbation of the magnetic field $\mathbf{B}_{1}\left(B_{\mathrm{r}}, B_{\theta}, B_{\varphi}\right)$, decomposed in poloidal and toroidal scalars $\left(B_{\mathrm{p}}, B_{\mathrm{t}}\right) . \Omega_{\mathrm{ficb}}$ and $\Omega_{\mathrm{fcmb}}$ are the angular velocities of the fluid core related to the perturbed velocity field $\mathbf{u}_{1}$ at ICB and CMB, and $\Omega_{\mathrm{ic}}$ and $\Omega_{\mathrm{m}}$ are the perturbations of the angular velocities of rotation of the inner core and the mantle, respectively. The radii $r_{\mathrm{i}}$ and $r_{\mathrm{o}}$ are those of the inner core and the core and $\Delta$ is the thickness of the electrically conducting layer at the base of the mantle.

and applying this decomposition to the time-dependent electromagnetic torque expression leads to

$$
\Gamma_{\mathrm{ICB}}(t)=\Gamma_{\mathrm{ICB}}^{0}+\Gamma_{\mathrm{ICB}}^{1}(t),
$$

with

$$
\Gamma_{\mathrm{ICB}}^{0}=\frac{r_{\mathrm{i}}}{\mu_{0}} \int_{S_{\mathrm{ICB}}} B_{\mathrm{r}}^{0} B_{\varphi}^{0} \sin \theta \mathrm{dS},
$$

the order one torque, resulting from the interaction between the radial and azimuthal components of the main magnetic field $\mathbf{B}_{0}$. This decomposition assumes that the product between the azimuthal and radial parts of the perturbed field $\mathbf{B}_{1}$ is negligible and states that the prevailing time variations in the electromagnetic torques derive from the product between the radial component of the main field and the azimuthal perturbations at the fluid core boundaries like

$$
\Gamma_{\mathrm{ICB}}^{1}(t)=\frac{r_{\mathrm{i}}}{\mu_{0}} \int_{S_{\mathrm{ICB}}} B_{\mathrm{r}}^{0} B_{\varphi}(t) \sin \theta \mathrm{dS},
$$

and equivalently

$$
\Gamma_{\mathrm{CMB}}^{1}(t)=-\frac{r_{\mathrm{o}}}{\mu_{0}} \int_{S_{\mathrm{CMB}}} B_{\mathrm{r}}^{0} B_{\varphi}(t) \sin \theta \mathrm{dS},
$$

at the core-mantle boundary. Equations (5.7) and (5.8) clearly show that a time-dependent descriptions of the electromagnetic torques necessarily involves a time-dependent description of the azimuthal perturbations of the magnetic field $B_{\varphi}(t)$ at the fluid core boundaries. Such 
a description can be achieved by, firstly, solving the evolution of this perturbation away from the boundaries.

In the inner core, fluid outer core and the electrically conducting part of the mantle, the magnetic field obeys the general induction equation,

$$
\frac{\partial \mathbf{B}}{\partial t}=\nabla \times(\mathbf{u} \times \mathbf{B})-\nabla \times(\eta \nabla \times \mathbf{B}) .
$$

Using the decomposition introduced by the system (5.3), and neglecting the second order term $\left[\boldsymbol{\nabla} \times\left(\mathbf{u}_{1} \times \mathbf{B}_{1}\right)\right]$ we have

$$
\begin{aligned}
\frac{\partial \mathbf{B}_{0}}{\partial t}+\frac{\partial \mathbf{B}_{1}}{\partial t} & =\nabla \times\left(\mathbf{u}_{0} \times \mathbf{B}_{0}\right)+\nabla \times\left(\mathbf{u}_{0} \times \mathbf{B}_{1}\right)+\nabla \times\left(\mathbf{u}_{1} \times \mathbf{B}_{0}\right) \\
& -\nabla \times\left(\eta \boldsymbol{\nabla} \times \mathbf{B}_{0}\right)-\boldsymbol{\nabla} \times\left(\eta \nabla \times \mathbf{B}_{1}\right)
\end{aligned}
$$

At this point, we aim at simplifying equation (5.10) in order to obtain a linearized induction equation that will describe the evolution of the perturbed field $\mathbf{B}_{1}$. To do so, we first use the fact that the main magnetic field $\mathbf{B}_{0}$ is slowly varying over time, so that

$$
\frac{\partial \mathbf{B}_{0}}{\partial t} \approx 0
$$

This is in agreement with the balance between the advection and the diffusion of the main, large-scale, magnetic field

$$
\left|\boldsymbol{\nabla} \times\left(\mathbf{u}_{0} \times \mathbf{B}_{0}\right)\right| \simeq\left|\nabla \times\left(\eta \boldsymbol{\nabla} \times \mathbf{B}_{0}\right)\right|
$$

Equation (5.10) can then be written

$$
\frac{\partial \mathbf{B}_{1}}{\partial t}=\nabla \times\left(\mathbf{u}_{0} \times \mathbf{B}_{1}\right)+\nabla \times\left(\mathbf{u}_{1} \times \mathbf{B}_{0}\right)-\nabla \times\left(\eta \nabla \times \mathbf{B}_{1}\right)
$$

in which the advection terms can be developed like

$$
\boldsymbol{\nabla} \times\left(\mathbf{u}_{0} \times \mathbf{B}_{1}\right)=\left(\boldsymbol{\nabla} \cdot \mathbf{B}_{1}\right) \mathbf{u}_{0}-\left(\boldsymbol{\nabla} \cdot \mathbf{u}_{0}\right) \mathbf{B}_{1}+\left(\mathbf{B}_{1} \cdot \boldsymbol{\nabla}\right) \mathbf{u}_{0}-\left(\mathbf{u}_{0} \cdot \boldsymbol{\nabla}\right) \mathbf{B}_{1},
$$

and

$$
\boldsymbol{\nabla} \times\left(\mathbf{u}_{1} \times \mathbf{B}_{0}\right)=\left(\boldsymbol{\nabla} \cdot \mathbf{B}_{0}\right) \mathbf{u}_{1}-\left(\boldsymbol{\nabla} \cdot \mathbf{u}_{1}\right) \mathbf{B}_{0}+\left(\mathbf{B}_{0} \cdot \boldsymbol{\nabla}\right) \mathbf{u}_{1}-\left(\mathbf{u}_{1} \cdot \boldsymbol{\nabla}\right) \mathbf{B}_{0},
$$

respectively. As the main and perturbed fields are solenoidal fields,

$$
\begin{array}{ll}
\boldsymbol{\nabla} \cdot \mathbf{B}_{0}=0, & \boldsymbol{\nabla} \cdot \mathbf{B}_{1}=0 \\
\nabla \cdot \mathbf{u}_{0}=0, & \boldsymbol{\nabla} \cdot \mathbf{u}_{1}=0
\end{array}
$$


equation (5.13) becomes

$$
\begin{aligned}
\frac{\partial \mathbf{B}_{1}}{\partial t} & =\left(\mathbf{B}_{1} \cdot \boldsymbol{\nabla}\right) \mathbf{u}_{0}-\left(\mathbf{u}_{0} \cdot \boldsymbol{\nabla}\right) \mathbf{B}_{1}+\left(\mathbf{B}_{0} \cdot \boldsymbol{\nabla}\right) \mathbf{u}_{1} \\
& -\left(\mathbf{u}_{1} \cdot \boldsymbol{\nabla}\right) \mathbf{B}_{0}-\boldsymbol{\nabla} \times\left(\eta \boldsymbol{\nabla} \times \mathbf{B}_{1}\right) .
\end{aligned}
$$

In order to obtain an analytical expression for the evolution of the perturbed field $\mathbf{B}_{1}$, we now wish to extract the main source term from equation (5.18). To this end, we first assume that the terms involving the gradients of the main velocity and magnetic fields are small respective to the other source terms. Indeed, as $\mathbf{u}_{0}$ and $\mathbf{B}_{0}$ are considered as large-scale fields in our analysis, their respective gradients should scale as $D^{-1}$ and can thus be neglected. One last argument lies in the fact that the major part of our work is based on stress-free conditions for the velocity field at the fluid outer core boundaries, consistently with the assumption that the radial gradient of $\mathbf{u}_{1}$ is the largest source term in equation (5.18). These examinations on the different advective terms imply that

$$
\left(\mathbf{B}_{1} \cdot \nabla\right) \mathbf{u}_{0},\left(\mathbf{u}_{1} \cdot \nabla\right) \mathbf{B}_{0} \ll\left(\mathbf{u}_{0} \cdot \nabla\right) \mathbf{B}_{1}<\left(\mathbf{B}_{0} \cdot \nabla\right) \mathbf{u}_{1}
$$

The identified source term at both ICB and CMB can be pictured as two velocity jumps (see Fig. 5.1), that will appear in the interface conditions of the magnetic and electric fields in section 5.2. They are namely the differences between the angular velocity of the fluid close to ICB and CMB, $\Omega_{\text {ficb }}$ and $\Omega_{\mathrm{fcmb}}$, and the perturbations of solid body rotation of the inner core $\Omega_{\text {ic }}$ and the mantle $\Omega_{\mathrm{m}}$. The generation of the perturbed magnetic field $\mathbf{B}_{1}$ is governed by the velocity jumps at either ICB or $\mathrm{CMB}$, and the diffusion of this field into the inner core, the fluid outer core and the electrically conducting part of the mantle. However, away from the fluid core boundaries, the perturbed magnetic field $\mathbf{B}_{1}$ is no longer sensitive to these velocity jumps, and will thus diffuse away into a layer of electrical conductivity $\sigma$, magnetic diffusivity $\eta$ and thickness $\Delta$ according to

$$
\frac{\partial \mathbf{B}_{1}}{\partial t}=-\nabla \times\left(\eta \nabla \times \mathbf{B}_{1}\right) .
$$

We now intend to solve this diffusion equation into the different media pictured in figure5.1. In order to investigate de frequency response of the perturbed field and of the electromagnetic torques, this will be performed with a harmonic analysis. We thus look for analytic expression of $\widetilde{\mathbf{B}}_{1}$, the Fourier transform of $\mathbf{B}_{1}$ like,

$$
\widetilde{\mathbf{B}}_{1}(\omega)=\frac{1}{\sqrt{2 \pi}} \int_{-\infty}^{+\infty} \mathbf{B}_{1}(t) e^{-i \omega t} \mathrm{dt}
$$

where $\omega$ is the angular frequency. The azimuthal component of perturbed field, $\widetilde{B}_{\varphi}$, enters 
the Fourier transforms of the electromagnetic torques as

$$
\widetilde{\Gamma}_{\mathrm{ICB}}^{1}(\omega)=\frac{r_{\mathrm{i}}}{\mu_{0}} \int_{S_{\mathrm{ICB}}} B_{\mathrm{r}}^{0} \widetilde{B}_{\varphi}(\omega) \sin \theta \mathrm{dS}
$$

and equivalently

$$
\widetilde{\Gamma}_{\mathrm{CMB}}^{1}(\omega)=-\frac{r_{\mathrm{o}}}{\mu_{0}} \int_{S_{\mathrm{CMB}}} B_{\mathrm{r}}^{0} \widetilde{B}_{\varphi}(\omega) \sin \theta \mathrm{dS}
$$

which are defined as

$$
\widetilde{\Gamma}_{\mathrm{ICB}}^{1}(\omega)=\frac{1}{\sqrt{2 \pi}} \int_{-\infty}^{+\infty} \Gamma_{\mathrm{ICB}}^{1}(t) e^{-i \omega t} \mathrm{dt}
$$

and

$$
\widetilde{\Gamma}_{\mathrm{CMB}}^{1}(\omega)=\frac{1}{\sqrt{2 \pi}} \int_{-\infty}^{+\infty} \Gamma_{\mathrm{CMB}}^{1}(t) e^{-i \omega t} \mathrm{dt}
$$

The description of $\widetilde{\mathbf{B}}_{1}$ will be performed using a poloidal/toroidal decomposition of the perturbed magnetic field, that is expressed as

$$
\widetilde{\mathbf{B}}_{1}=\nabla \times \nabla \times\left(\mathbf{r} \widetilde{B}_{\mathrm{p}}\right)+\nabla \times\left(\mathbf{r} \widetilde{B}_{\mathrm{t}}\right)
$$

with $\mathbf{r}$ the radius vector. As the demonstration is focused on the evolution of $\widetilde{\mathbf{B}}_{1}$, this decomposition gets rid of the subscripts distinguishing the main and perturbed fields, substantially facilitating the display of equations. In the following, as in the numerical implementation, the poloidal and toroidal scalars are expanded in spherical harmonics like

$$
\begin{aligned}
\widetilde{B}_{\mathrm{t}}(\omega) & =\sum_{\ell=0}^{\mathrm{L}_{\max }} \sum_{m=0}^{\ell} \widetilde{B}_{\mathrm{t}_{\ell}}^{m}(r, \omega) Y_{\ell}^{m}(\theta, \varphi), \\
\widetilde{B}_{\mathrm{p}}(\omega) & =\sum_{\ell=0}^{\mathrm{L}_{\max }} \sum_{m=0}^{\ell} \widetilde{B}_{\mathrm{p} \ell}^{m}(r, \omega) Y_{\ell}^{m}(\theta, \varphi),
\end{aligned}
$$

where $Y_{\ell}^{m}$ is a spherical harmonic function of order $m$ and degree $\ell$. For a given pair of degree and order of the spherical harmonic expansion, equation (5.20) becomes a set of two differential equations of order 2 , describing the diffusion of the coefficients of expansion of the poloidal and toroidal scalars, $\widetilde{B}_{\mathrm{p}_{\ell}}^{m}(r, \omega)$ and $\widetilde{B}_{\mathrm{t}_{\ell}}^{m}(r, \omega)$, into the electrically conducting layer,

$$
\begin{aligned}
& \frac{\mathrm{d}^{2}\left(r \widetilde{B}_{\mathrm{t}_{\ell}}^{m}\right)}{\mathrm{d} r^{2}}+\frac{1}{\eta} \frac{\mathrm{d} \eta}{\mathrm{d} r} \frac{\mathrm{d}\left(r \widetilde{B}_{\mathrm{t}_{\ell}}^{m}\right)}{\mathrm{d} r}+ {\left[\frac{i \omega}{\eta}-\frac{\ell(\ell+1)}{r^{2}}\right]\left(r \widetilde{B}_{\mathrm{t}_{\ell}}^{m}\right)=0, } \\
& \frac{\mathrm{d}^{2}\left(r \widetilde{B}_{\mathrm{p}_{\ell}}^{m}\right)}{\mathrm{d} r^{2}}+\left[\frac{i \omega}{\eta}-\frac{\ell(\ell+1)}{r^{2}}\right]\left(r \widetilde{B}_{\mathrm{p}_{\ell}}^{m}\right)=0 .
\end{aligned}
$$

Equations (5.29) and (5.30) form the basis of the following subsections, that are dedicated to particular solutions of these equations in the conducting part of the mantle, the fluid outer core and the inner core. This is a mandatory step, since the link between these 
solutions at the fluid core boundaries will define the behavior of $\widetilde{B}_{\varphi}(\omega)$ at ICB and CMB.

\subsubsection{Mantle-side: Constant conductivity}

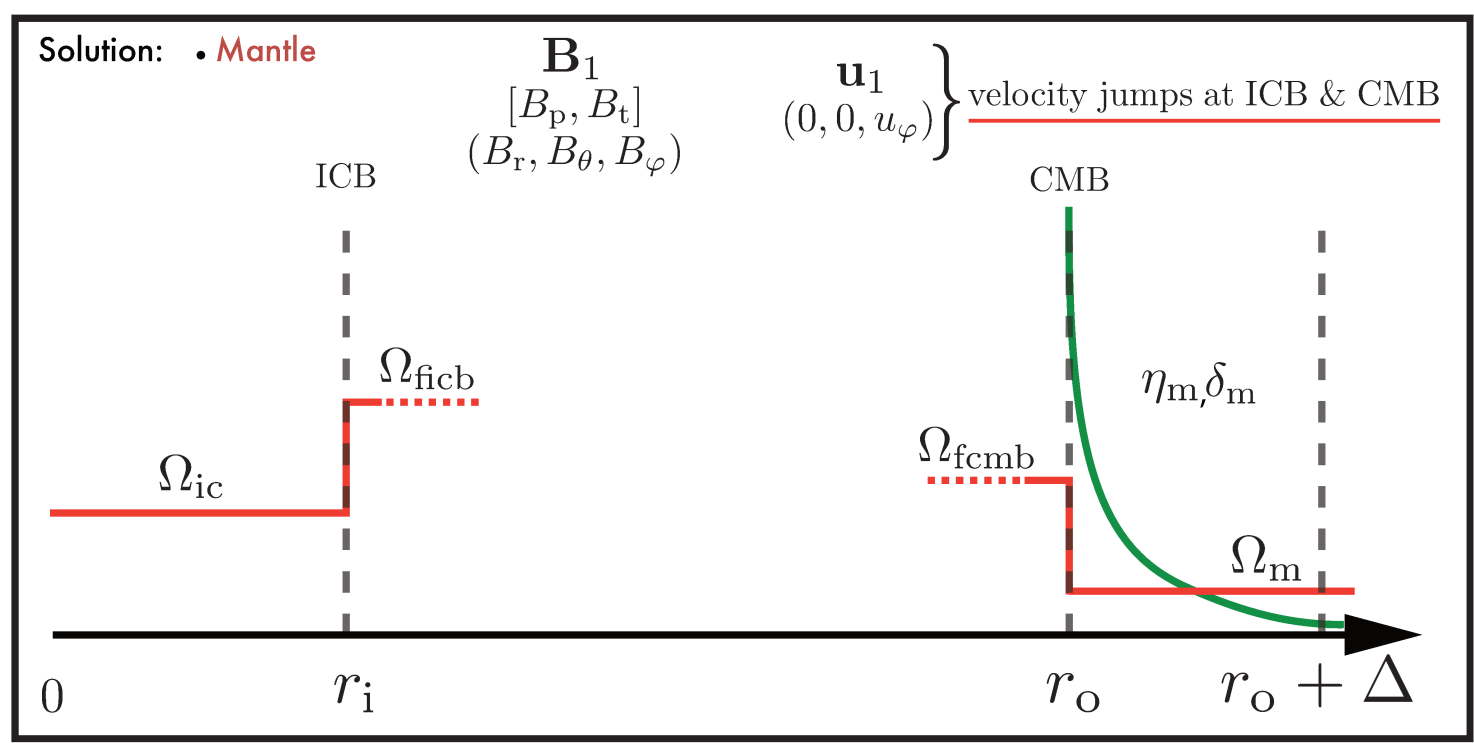

Figure 5.2: Same as figure 5.1, now with an approximate solution (with exact expression defined by equation (5.39)) of the evolution of the toroidal part of the perturbed magnetic field diffusing into the conducting part of the mantle, in green. The magnetic diffusivity of the conducting layer is $\eta_{\mathrm{m}}=1 / \mu_{0} \sigma_{\mathrm{m}}$, with $\sigma_{\mathrm{m}}$ the electrical conductivity, and $\delta_{\mathrm{m}}=\sqrt{2 \eta_{\mathrm{m}} / \omega}$ is the skin depth of the mantle for a signal of angular frequency $\omega$.

We first aim at determining the magnetic field diffusing into a thin electrically conducting layer at the base of the mantle. The electrical conductivity of the layer is noted $\sigma_{\mathrm{m}}$ and its magnetic diffusivity $\eta_{\mathrm{m}}$. The layer is located between radii $r_{\mathrm{o}}$ and $r_{\mathrm{o}}+\Delta$, with $r_{\mathrm{o}}$ the radius of the core and $\Delta$ the thickness of the layer. Moreover, this layer is assumed to have a constant conductivity profile (i.e. $\partial \eta_{\mathrm{m}} / \partial r=0$ ). In the following demonstration, we will also assume that the radial variations of $\widetilde{B}_{\mathrm{t}_{\ell}}^{m}$ and $\widetilde{B}_{\mathrm{p}_{\ell}}^{m}$ are large when compared to the horizontal variations, meaning that

$$
\frac{\omega}{\eta_{\mathrm{m}}} \gg \frac{\ell(\ell+1)}{r^{2}}
$$

The validity domain of this assumption is shown by figure 5.3. For a thin layer having the conductivity of the core (Fig. 5.3a), we observe that this assumption holds for $\omega \tau_{\eta}>5 \times 10^{3}$, with $\tau_{\eta}=D^{2} / \eta_{\mathrm{c}}$ the diffusive time of the core, corresponding to periods lower than 385 years, and spherical harmonic degree $\ell<100$. At smaller horizontal scales (i.e. higher harmonic degree, $\ell=1000)$, the validity of this assumption is disrupted if $\omega \tau_{\eta} \ll 4 \times 10^{5}$, that is for periods higher than 4.8 years. If the conductance of the lower mantle is embodied by a larger layer with a lower electrical conductivity (Fig. 5.3b), neglecting the horizontal variations in comparison with the radial ones is more critical. The assumption holds for the 

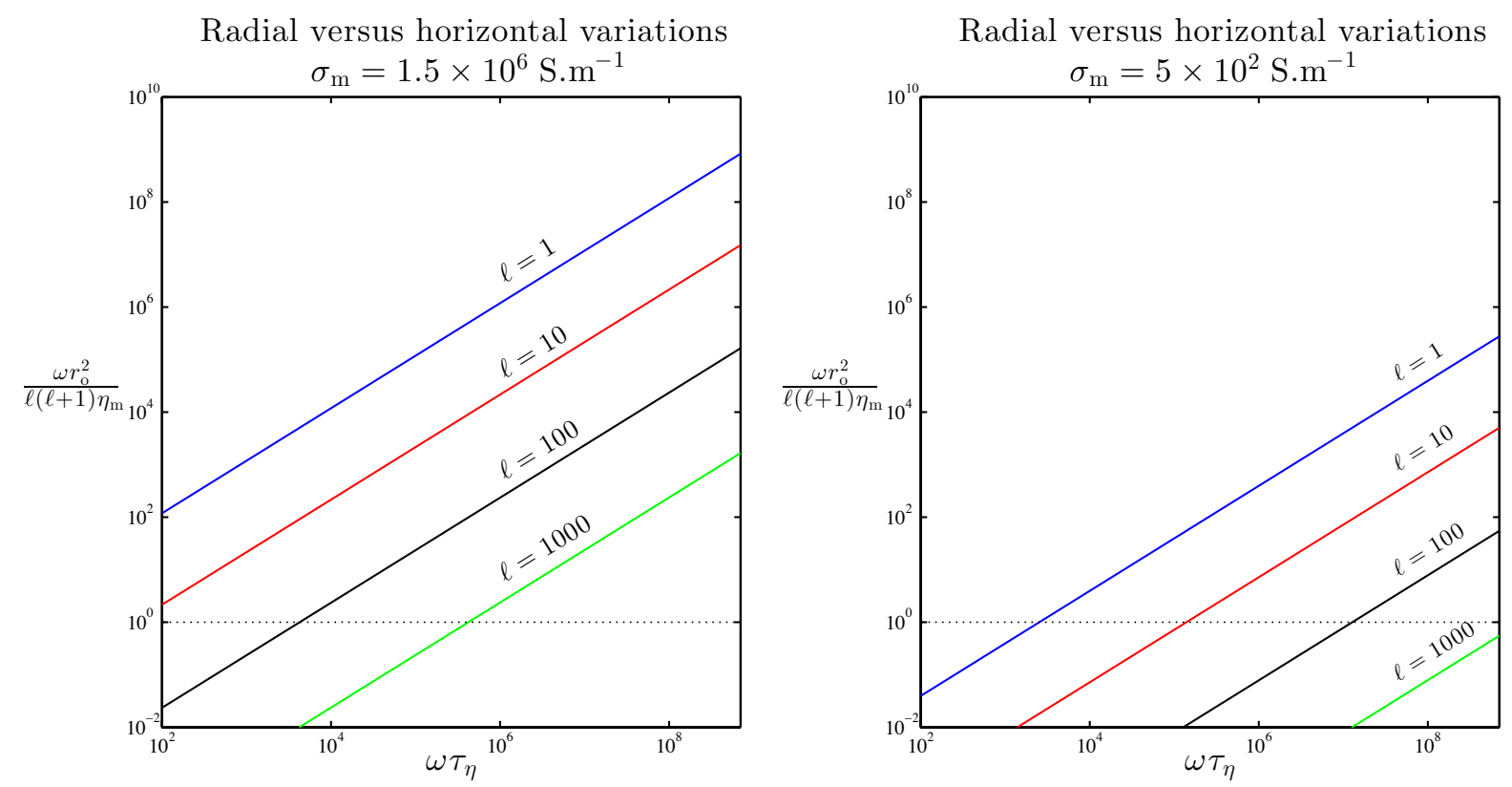

(a) For a thin layer of 150 meters having the (b) For a thick layer of 200 kilometers of postelectrical conductivity of the core, $\sigma_{\mathrm{m}}=\sigma_{\mathrm{c}}=$ perovskite with electrical conductivity $\sigma_{\mathrm{m}}=$ $1.5 \times 10^{6}{\mathrm{~S} . \mathrm{m}^{-1}}^{-1} \quad 5 \times 10^{2}{\mathrm{~S} . \mathrm{m}^{-1}}^{-1}$

Figure 5.3: Relative amplitude between horizontal and radial variations of the magnetic field as function of the angular frequency of the signal $\omega$ normalized by the characteristic time of magnetic diffusion in the core $\tau_{\eta}=D^{2} / \eta_{\mathrm{c}}$, for $r=r_{\mathrm{o}}$ the radius of the core, for different spherical harmonic degree $\ell$. Cases $5.3 \mathrm{a}$ and $5.3 \mathrm{~b}$ stand for two possible electrical conductivity distributions in the mantle, both leading to a lower mantle conductance of $10^{8} \mathrm{~S}$.

largest scales and periods lower than 385 years, but is no longer valid for large harmonic degree, especially for period longer than the day (i.e. $\omega \tau_{\eta} \mapsto 7 \times 10^{8}$ ).

Within the framework where this assumption is strictly valid and a constant conductivity profile, equations (5.29) and (5.30) picture no dependence on $\ell$ or $m$ and respectively become

$$
\begin{aligned}
& \frac{\mathrm{d}^{2}\left(r \widetilde{B}_{\mathrm{t}_{\ell}}^{m}\right)}{\mathrm{d} r^{2}}+\frac{i \omega}{\eta_{\mathrm{m}}}\left(r \widetilde{B}_{\mathrm{t}_{\ell}}^{m}\right)=0, \text { and } \\
& \frac{\mathrm{d}^{2}\left(r \widetilde{B}_{\mathrm{p}_{\ell}}^{m}\right)}{\mathrm{d} r^{2}}+\frac{i \omega}{\eta_{\mathrm{m}}}\left(r \widetilde{B}_{\mathrm{p}_{\ell}}^{m}\right)=0 .
\end{aligned}
$$

both written as

$$
\frac{\mathrm{d}^{2} X}{\mathrm{~d} r^{2}}+\frac{2 i}{\delta_{\mathrm{m}}^{2}} X=0, \quad \text { with } X=r \widetilde{B}_{\mathrm{t}_{\ell}}^{m} \text { or } X=r \widetilde{B}_{\mathrm{p}_{\ell}}^{m},
$$

using the standard definition of a skin depth,

$$
\delta_{\mathrm{m}}=\sqrt{\frac{2 \eta_{\mathrm{m}}}{\omega}} .
$$

Equation (5.34) is a homogeneous, second order, linear equation with a complex coefficient 
that has solutions of the type

$$
X=A e^{\left(\frac{1-i}{\delta_{\mathrm{m}}}\right) r}+B e^{-\left(\frac{1-i}{\delta_{\mathrm{m}}}\right) r},
$$

with $A$ and $B$ two constants to be determined. Even though $X$ describes the diffusion of both the poloidal and toroidal part of the magnetic field into the layer, we show in section 5.2.1 that providing a particular solution for $\widetilde{B}_{\mathrm{p}_{\ell}}^{m}$ is not mandatory. We thus focus on an exact solution for the toroidal part, i.e. $X=r \widetilde{B}_{\mathrm{t}_{\ell}}^{m}$. The constants $A$ and $B$ can be defined using the boundary conditions on the toroidal scalar of the magnetic field. The continuity of toroidal magnetic field in $r_{\mathrm{o}}$, in the absence of surface currents in the system (see A.1), provides the first boundary condition, in $r_{\mathrm{o}}$

$$
X\left(r_{\mathrm{o}}\right)=\bar{X}
$$

with $\bar{X}$ the value of the toroidal scalar at the core mantle boundary. In $r_{\mathrm{o}}+\Delta$ the toroidal part of the magnetic field is also continuous. However, as the mantle is considered as insulating beyond the electrically conducting layer, the field matches a potential field such that $\widetilde{B}_{\mathrm{t}_{\ell}}^{m}$ must be equal to zero on either sides of $r_{\mathrm{o}}+\Delta$. This yields,

$$
X\left(r_{\mathrm{o}}+\Delta\right)=0
$$

Applying the boundary conditions to equation (5.36) (see demonstration A.2) eventually leads to

$$
\frac{X}{\bar{X}}\left(r>r_{\mathrm{o}}, \omega\right)=\frac{\sinh \left[\left(\frac{1-i}{\delta_{\mathrm{m}}}\right)\left(r_{\mathrm{o}}+\Delta-r\right)\right]}{\sinh \left[\left(\frac{1-i}{\delta_{\mathrm{m}}}\right) \Delta\right]} .
$$

Figure 5.4 shows the absolute value and the argument (phase lag) of the toroidal part of the perturbation of the magnetic field diffusing into the electrically conducting part of the lower mantle defined by equation (5.39). In the two cases the electrical conductivity is constant, but the conductance is either embodied by a thin layer of 150 meters having the conductivity of the core or a layer of 200 kilometers with $\sigma_{\mathrm{m}}=5 \times 10^{2} \mathrm{~S}_{\mathrm{m}}{ }^{-1}$. In both cases, the toroidal field decreases rapidly to satisfy the condition $B_{\mathrm{t}}\left(r_{\mathrm{o}}+\Delta\right)=0$, and the shorter the period of the signal, the faster the toroidal field decreases (Fig. 5.4). For the very thin layer case (Fig. 5.4a \& Fig. 5.4b), the solution loses the dependency on the signal period if the period is greater than a few days. Indeed, the field decreases linearly from its value in $r_{\mathrm{o}}$, to zero in $r_{\mathrm{o}}+\Delta$ (Fig. 5.4a) while the phase lag approaches a vanishing constant as the frequency is increased. For a thicker layer with a lower electrical conductivity, as shown by figures $5.4 \mathrm{c}$ and $5.4 \mathrm{~d}$, the same behavior can be noticed for periods greater than a few years. However, the perturbed magnetic field diffusing in such a layer is more sensitive on signals having periods lower than six months. This will have several consequences for the determination and the behavior of the perturbed magnetic field at the CMB, discussed 


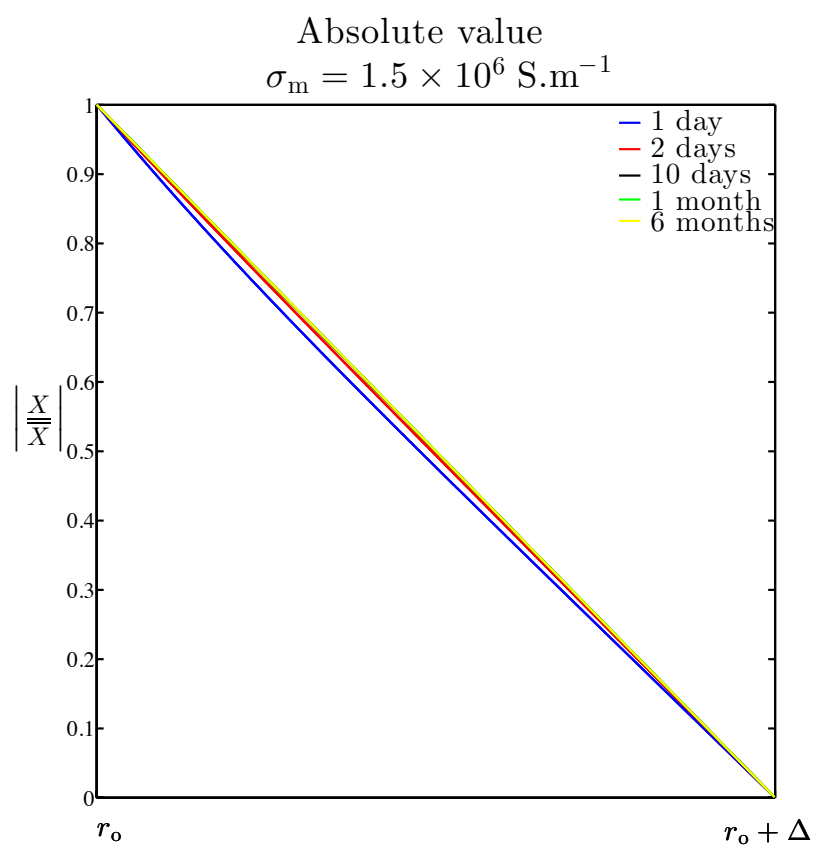

(a)

Absolute value

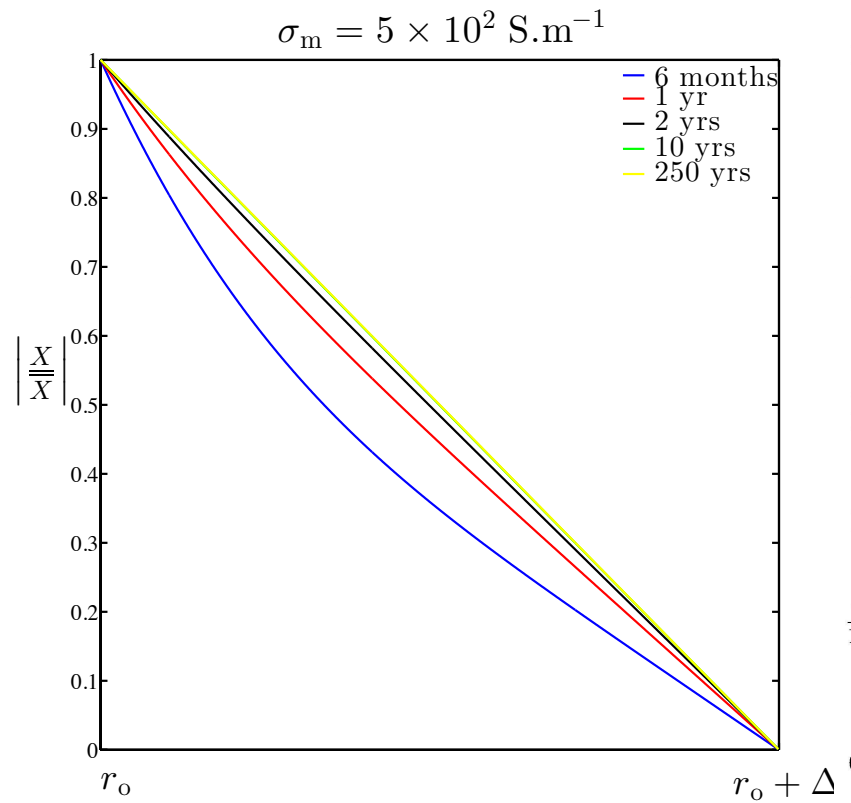

(c)

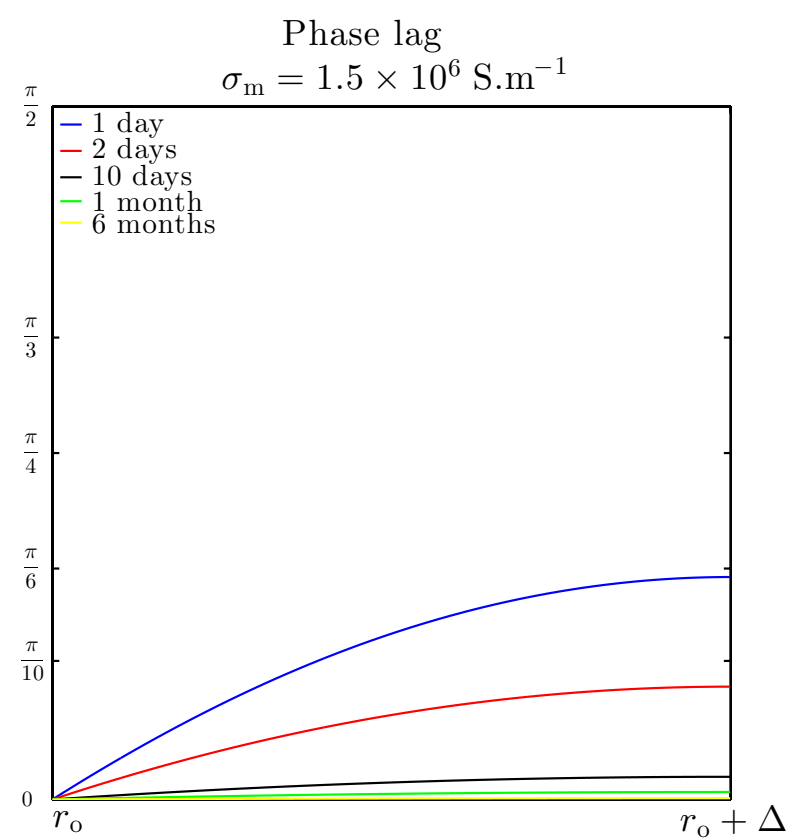

(b)

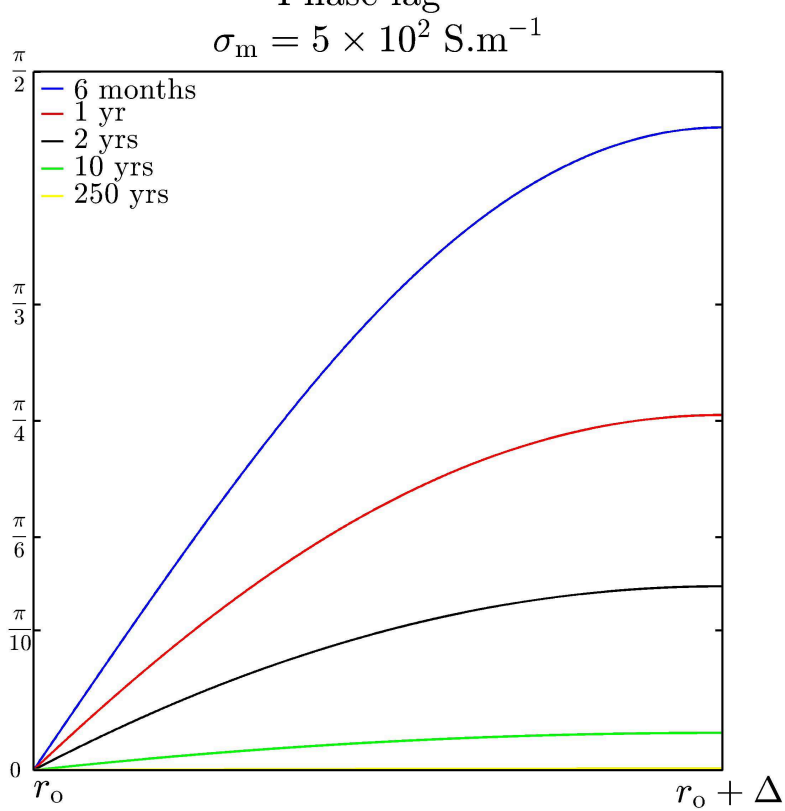

(d)

Figure 5.4: Absolute value and the phase of solution (5.39) of the toroidal part of the magnetic field diffusing into the electrically conducting part of the mantle, for two possible electrical conductivity distributions in the mantle, both leading to a lower mantle conductance of $10^{8} \mathrm{~S}$, and for different signal periods. The first case, figures (a) and (b), stands for a thin layer with $\Delta=150 \mathrm{~m}$, having the electrical conductivity of the core, $\sigma_{\mathrm{m}}=\sigma_{\mathrm{c}}=1.5 \times 10^{6} \mathrm{~S}_{\mathrm{m}} \mathrm{m}^{-1}$, while the second case, figures (c) and (d), pictures a thicker layer of $\Delta=200 \mathrm{~km}$ of post-perovskite with electrical conductivity $\sigma_{\mathrm{m}}=5 \times 10^{2} \mathrm{~S}_{\mathrm{m}}{ }^{-1}$.

in section 5.2.4. 


\subsubsection{Fluid core side}

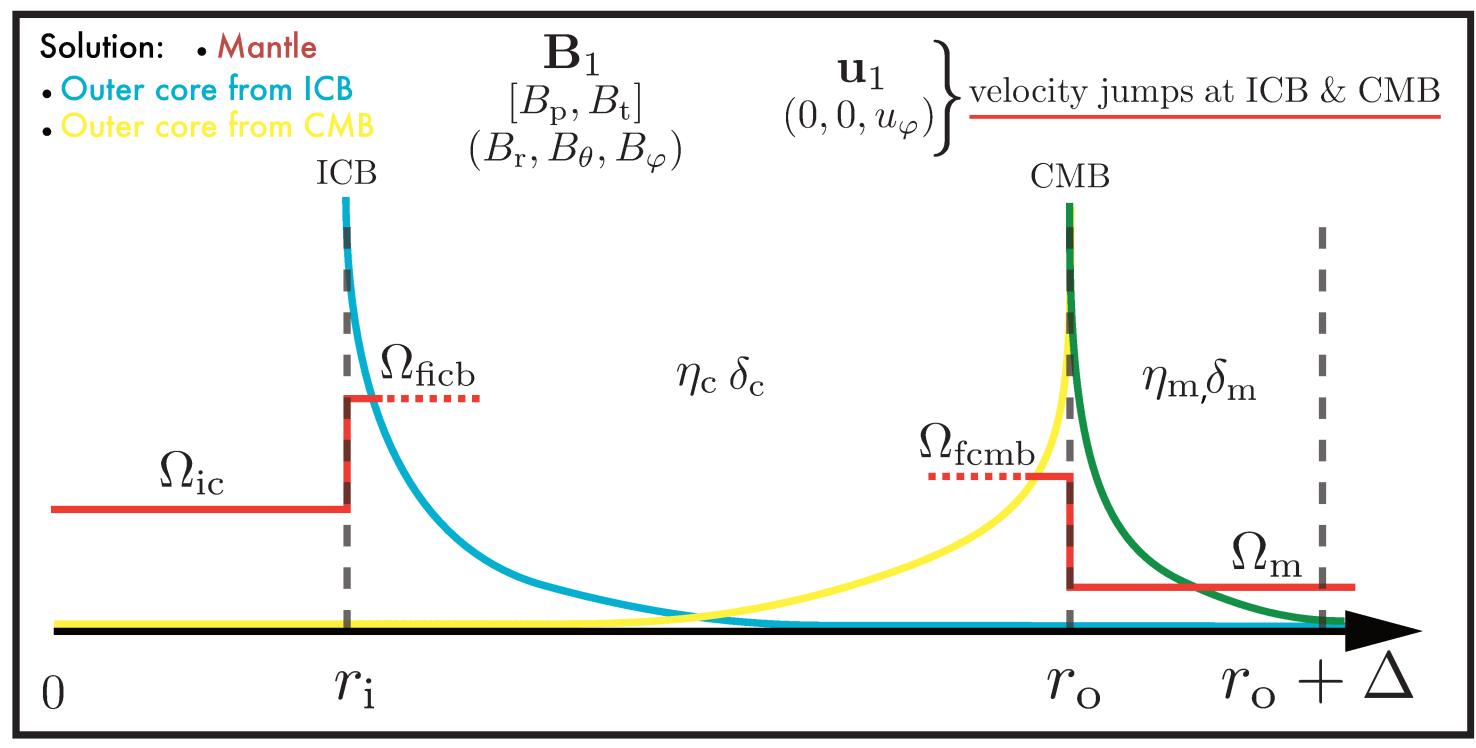

Figure 5.5: Same as figure 5.2, now with two approximate solutions (with exact expressions defined by equations (5.45) and (5.49))of the evolution of the toroidal part of the perturbed magnetic field diffusing from the CMB into the core, in yellow, and from the ICB towards infinity, in blue. The magnetic diffusivity of both the fluid outer core and the inner core is $\eta_{\mathrm{c}}=1 / \mu_{0} \sigma_{\mathrm{c}}$, with $\sigma_{\mathrm{c}}$ the electrical conductivity of the core, and $\delta_{\mathrm{c}}=\sqrt{2 \eta_{\mathrm{c}} / \omega}$ is a typical skin depth in the core for a signal of angular frequency $\omega$.

We now aim at determining the magnetic field $\widetilde{\mathbf{B}}_{1}$ produced at the fluid core boundaries, that will diffuse away from the CMB into the core, and from the ICB into the fluid outer core. We consider that the fluid core and the inner core have the same, constant, electrical conductivity $\sigma_{\mathrm{c}}$, so that the solutions for both the poloidal and toroidal parts of the magnetic field are of the form

$$
X=A_{c} e^{\left(\frac{1-i}{\delta_{c}}\right) r}+B_{c} e^{-\left(\frac{1-i}{\delta_{c}}\right) r}, \quad \text { with } X=r \widetilde{B}_{\mathrm{t}_{\ell}}^{m} \text { or } X=r \widetilde{B}_{\mathrm{p} \ell}^{m},
$$

with $A_{c}$ and $B_{c}$ two constants, and

$$
\delta_{\mathrm{c}}=\sqrt{\frac{2 \eta_{\mathrm{c}}}{\omega}}
$$

a typical skin depth in the core. In the fluid core, we require that the perturbation $\widetilde{\mathbf{B}}_{1}$ triggered by the velocity jump vanishes away from the boundary.

\section{At the CMB}

At the core-mantle boundary, the condition that $\widetilde{\mathbf{B}}_{1}$ vanishes away from the boundary,

$$
\frac{r-r_{\mathrm{o}}}{\delta_{\mathrm{c}}} \mapsto-\infty, \quad \text { necessarily involves } B_{c}=0 .
$$


The second condition is anew on the value of the magnetic field at the CMB $\left(r=r_{\mathrm{o}}\right)$, we have

$$
X\left(r_{\mathrm{o}}\right)=\bar{X}=A_{c} e^{\left(\frac{1-i}{\delta_{\mathrm{c}}}\right) r_{\mathrm{o}}}
$$

leading to

$$
A_{c}=\bar{X} e^{-\left(\frac{1-i}{\delta_{\mathrm{c}}}\right) r_{\mathrm{o}}}
$$

The association of the two boundary conditions (Eq. 5.42 \& Eq. 5.44) gives the general solution

$$
\frac{X}{\bar{X}}\left(r<r_{\mathrm{o}}, \omega\right)=e^{\left(\frac{1-i}{\delta_{\mathrm{c}}}\right)\left(r-r_{\mathrm{o}}\right)},
$$

that we recall is valid for

$$
X=r \widetilde{B}_{\mathrm{p}_{\ell}}^{m}, \quad \text { with } \quad \bar{X}=r_{\mathrm{o}} \widetilde{B}_{\mathrm{p}_{\ell}}^{m}\left(r_{\mathrm{o}}\right)
$$

and

$$
X=r \widetilde{B}_{\mathrm{t}_{\ell}}^{m}, \quad \text { with } \quad \bar{X}=r_{\mathrm{o}} \widetilde{B}_{\mathrm{t}_{\ell}}^{m}\left(r_{\mathrm{o}}\right)
$$

\section{At the ICB}

At the inner core boundary, the first condition to determine $A_{c}$ and $B_{c}$ is, for

$$
\frac{r-r_{\mathrm{o}}}{\delta_{\mathrm{c}}} \mapsto+\infty, \quad X=0
$$

and necessarily implies that $A_{c}=0$. Using the value of the magnetic field at the ICB $\left(r=r_{\mathrm{i}}\right)$, we have

$$
X\left(r_{\mathrm{i}}\right)=\underline{X}=B_{c} e^{-\left(\frac{1-i}{\delta_{\mathrm{c}}}\right) r_{\mathrm{i}}},
$$

leading to

$$
B_{c}=\underline{X} e^{\left(\frac{1-i}{\delta_{\mathrm{c}}}\right) r_{\mathrm{i}}}
$$

The combination between the two boundary conditions gives the general solution

$$
\underline{X} \underline{X}\left(r>r_{\mathrm{i}}, \omega\right)=e^{-\left(\frac{1-i}{\delta_{\mathrm{c}}}\right)\left(r-r_{\mathrm{i}}\right)},
$$

that is valid for

$$
X=r \widetilde{B}_{\mathrm{p}_{\ell}}^{m}, \quad \text { with } \quad \underline{X}=r_{\mathrm{i}} \widetilde{B}_{\mathrm{p}_{\ell}}^{m}\left(r_{\mathrm{i}}\right),
$$

and

$$
X=r \widetilde{B}_{\mathrm{t}_{\ell}}^{m}, \quad \text { with } \quad \underline{X}=r_{\mathrm{i}} \widetilde{B}_{\mathrm{t}_{\ell}}^{m}\left(r_{\mathrm{i}}\right) .
$$


Approximate representations of the exact solutions (5.45) and (5.49) can thus be added to figure 5.5, showing that at this point we only miss a solution of the diffusing magnetic field $\widetilde{\mathbf{B}}_{1}$ into the inner core.

\subsubsection{Inner core}

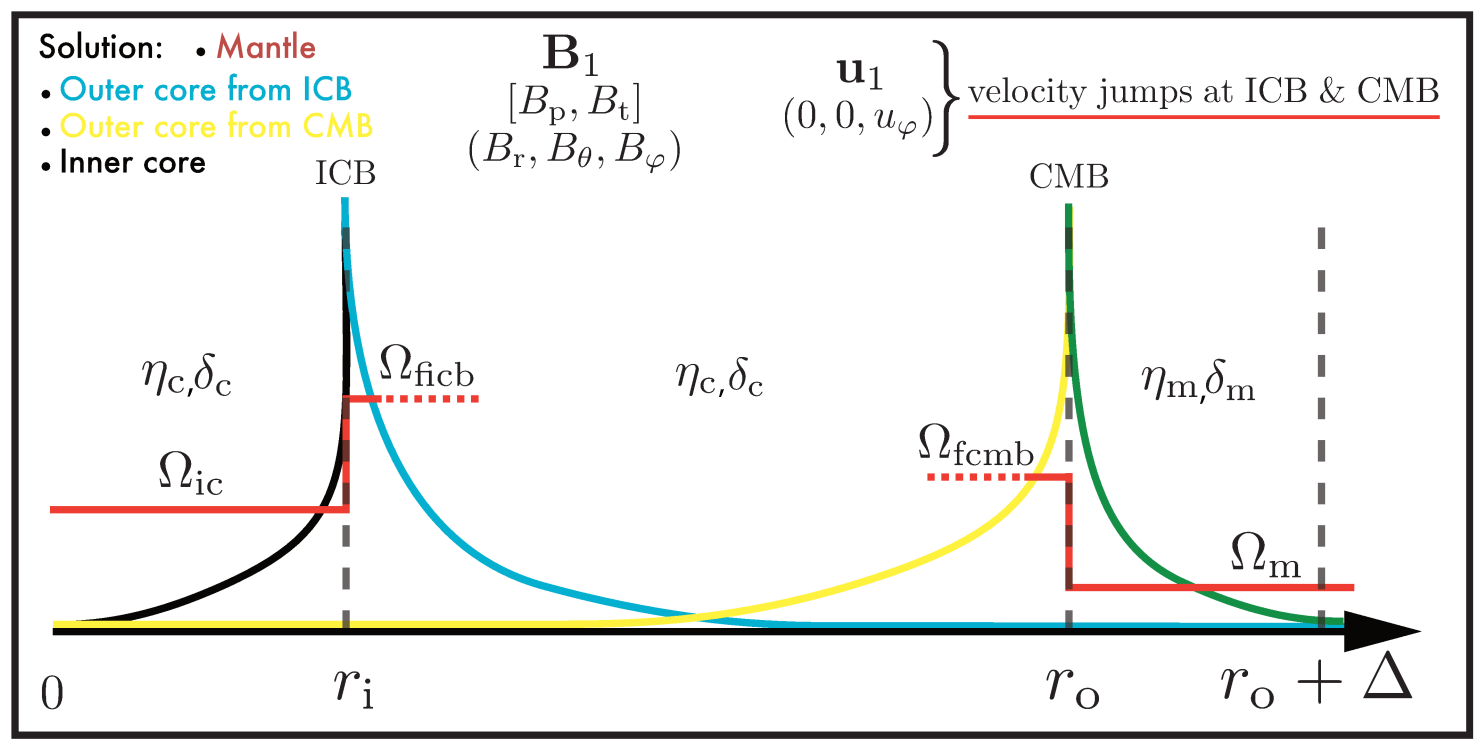

Figure 5.6: Same as 5.5, now with an approximate solution of the evolution of the toroidal part of the perturbed magnetic field diffusing from the ICB into the inner core, in black.

The situation in the inner core is analogous to that of the mantle-side, in the sense that the magnetic field diffuses into a layer of constant conductivity $\sigma_{\mathrm{c}}$. However, as the magnetic field diffuses deeper and deeper into the inner core, the assumption that the radial variation of $\mathbf{B}_{1}$ are greater than the horizontal (Eq. 5.31) ones can be defeated by $r \mapsto 0$. Restricting ourselves to the upper part of the inner core, the solution is again of the form

$$
X=A_{i c} e^{\left(\frac{1-i}{\delta_{c}}\right) r}+B_{i c} e^{-\left(\frac{1-i}{\delta_{c}}\right) r},
$$

with $A_{i c}$ and $B_{i c}$ two constants to be determined using the already defined interface conditions on the magnetic field. At the inner core boundary, the magnetic field is continuous and we will use its value at the ICB as a first condition, $X\left(r_{\mathrm{i}}\right)=\underline{X}$, leading to

$$
\underline{X}=A_{i c} e^{\left(\frac{1-i}{\delta_{c}}\right) r_{\mathrm{i}}}+B_{i c} e^{-\left(\frac{1-i}{\delta_{\mathrm{c}}}\right) r_{\mathrm{i}}} .
$$

Moreover, the condition that the magnetic should vanish away from the ICB into the inner core is equivalent to

$$
\frac{r-r_{\mathrm{i}}}{\delta_{\mathrm{c}}} \mapsto-\infty, \quad \text { and again involves } B_{c}=0
$$


The solution of the diffusion of both poloidal and toroidal parts of $\widetilde{\mathbf{B}}_{1}$ is then

$$
\frac{X}{\underline{X}}\left(r<r_{\mathrm{i}}, \omega\right)=e^{\left(\frac{1-i}{\delta_{\mathrm{c}}}\right)\left(r-r_{\mathrm{i}}\right)},
$$

for

$$
X=r \widetilde{B}_{\mathrm{p}_{\ell}}^{m}, \quad \text { with } \quad \underline{X}=r_{\mathrm{i}} \widetilde{B}_{\mathrm{p}_{\ell}}^{m}\left(r_{\mathrm{i}}\right),
$$

and

$$
X=r \widetilde{B}_{\mathrm{t}_{\ell}}^{m}, \quad \text { with } \quad \underline{X}=r_{\mathrm{i}} \widetilde{B}_{\mathrm{t}_{\ell}}^{m}\left(r_{\mathrm{i}}\right) .
$$

\section{$5.2 \quad$ Interface conditions}

\subsubsection{General case}

The previous section established the form of the perturbed field $\widetilde{\mathbf{B}}_{1}$ that will diffuse away from the fluid core boundaries as functions of their respective value at either ICB or CMB. In order to obtain analytic expressions of perturbed field at ICB and CMB, we henceforth have to link the solutions previously obtained. This can be achieved by considering the general interface conditions on an electromagnetic field (namely $\mathbf{E}$ and $\mathbf{B}$ ) crossing a boundary between two media. At either ICB or CMB, the conservation of the tangential part of the electric field $\mathbf{E}$ can be written

$$
\mathbf{r} \times\left.\mathbf{E}\right|_{\text {core }}=\mathbf{r} \times\left.\mathbf{E}\right|_{\text {ic } / \mathrm{m}}
$$

the subscripts ic and $\mathrm{m}$ standing for the inner core and the mantle, respectively. This equation can lead to a condition on the azimuthal magnetic field at the fluid core boundaries through the consecutive use of Ohm's law

$$
\mathbf{E}=\frac{\mathbf{J}}{\sigma_{\mathrm{c} / \mathrm{m}}}-\mathbf{u} \times \mathbf{B}
$$

with $\sigma_{\mathrm{c} / \mathrm{m}}$ the electrical conductivity of the core or the mantle, the inner core and the fluid outer core having the same value, and Ampère's law,

$$
\mu_{0} \mathbf{J}=\nabla \times \mathbf{B}
$$

The conservation of the tangential electric field (Eq. 5.56) then becomes

$$
\eta_{\mathrm{c}} \mathbf{r} \times \boldsymbol{\nabla} \times \mathbf{B}-\mathbf{r} \times \mathbf{u} \times\left.\mathbf{B}\right|_{\text {core }}=\eta_{\mathrm{c} / \mathrm{m}} \mathbf{r} \times \nabla \times \mathbf{B}-\mathbf{r} \times \mathbf{u} \times\left.\mathbf{B}\right|_{\mathrm{ic} / \mathrm{m}} .
$$

Applying this interface condition to the perturbation analysis developed in section 5.1.1 establish the direct relationship between the radial derivatives of the azimuthal magnetic 
field $B_{\varphi}$ on both sides of the fluid core boundaries, as a function of the velocity jump as

$$
\left.\eta_{\mathrm{c}} \frac{\partial r \widetilde{B}_{\varphi}}{\partial r}\right|_{\text {core }} ^{r_{\mathrm{i}} / r_{\mathrm{o}}}-\left.\eta_{\mathrm{c} / \mathrm{m}} \frac{\partial r \widetilde{B}_{\varphi}}{\partial r}\right|_{\mathrm{ic} / \mathrm{m}} ^{r_{\mathrm{i}} / r_{\mathrm{o}}}=\left.r_{\mathrm{i} / \mathrm{o}}^{2} B_{\mathrm{r}}^{0}\left(\Omega_{\mathrm{ficb} / \mathrm{fcmb}}-\Omega_{\mathrm{ic} / \mathrm{m}}\right)\right|_{\mathrm{ic} / \mathrm{m}} ^{r_{\mathrm{i}} / r_{\mathrm{o}}},
$$

with $r_{\mathrm{i} / \mathrm{o}}$ standing for the radius of the inner core $r_{\mathrm{i}}$ or the core $r_{\mathrm{o}}$. Also, as we have

$$
\frac{\partial r \widetilde{B}_{\varphi}}{\partial r}=\widetilde{B}_{\varphi}+r \frac{\partial \widetilde{B}_{\varphi}}{\partial r}
$$

and the continuity of the magnetic field at the interface,

$$
\left.\widetilde{B}_{\varphi}\right|_{\text {core }} ^{r_{\mathrm{i}} / r_{\mathrm{o}}}=\left.\widetilde{B}_{\varphi}\right|_{\mathrm{ic} / \mathrm{m}} ^{r_{\mathrm{i}} / r_{\mathrm{o}}}
$$

leads to a simplification of the discontinuity of the radial derivatives like

$$
\left.\eta_{\mathrm{c}} \frac{\partial \widetilde{B}_{\varphi}}{\partial r}\right|_{\text {core }} ^{r_{\mathrm{i}} / r_{\mathrm{o}}}-\left.\eta_{\mathrm{c} / \mathrm{m}} \frac{\partial \widetilde{B}_{\varphi}}{\partial r}\right|_{\mathrm{ic} / \mathrm{m}} ^{r_{\mathrm{i}} / r_{\mathrm{o}}}=\left.r_{\mathrm{i} / \mathrm{o}} B_{\mathrm{r}}^{0}\left(\Omega_{\mathrm{ficb} / \mathrm{fcmb}}-\Omega_{\mathrm{ic} / \mathrm{m}}\right)\right|_{\mathrm{ic} / \mathrm{m}} ^{r_{\mathrm{i}} / r_{\mathrm{o}}} .
$$

This expression can be used to obtain the form of the azimuthal magnetic field at the fluid core boundaries, considering the solutions of the toroidal and poloidal scalars of $\widetilde{\mathbf{B}}_{1}$ provided in section 5.1. To do so, we first express the radial derivative of $\widetilde{B}_{\varphi}$ in the framework of the poloidal/toroidal decomposition,

$$
\frac{\partial \widetilde{B}_{\varphi}}{\partial r}=\frac{\partial}{\partial r}\left[\frac{1}{\sin \theta} \frac{\partial}{\partial \phi}\left(\frac{1}{r} \frac{\partial r \widetilde{B}_{\mathrm{p}}}{\partial r}\right)-\frac{\partial \widetilde{B}_{\mathrm{t}}}{\partial \theta}\right] .
$$

The left part of this equation involves a radial derivative of $\widetilde{B}_{\mathrm{p}}$ of second order, while the right part only involves a first order derivative of $\widetilde{B}_{\mathrm{t}}$. In appendix A.1 we establish the continuity of poloidal field as well as its first and second radial derivatives across an interface between two electrically conducting media. We demonstrate that the second radial derivative of the poloidal field is continuous if, in our case,

$$
\left.\frac{\partial B_{\mathrm{r}}^{0} u_{\varphi}}{\partial \varphi}\right|_{\text {core }} ^{r_{\mathrm{i}} / r_{\mathrm{o}}}=0
$$

Even though this condition may be questionable in convective simulations of the geodynamo, it remains strictly valid in our analytical case as the perturbed field can be described as the result of the interaction between a mainly dipolar magnetic field $\mathbf{B}_{0}$ and an axisymmetric velocity jump at the fluid core boundary. We can thus anticipate that the discontinuity of the radial derivative of $\widetilde{B}_{\varphi}$ is indeed only carried by the discontinuity in the radial derivative 
of the toroidal field $B_{\mathrm{t}}$ such that,

$$
\begin{aligned}
-\left.\eta_{\mathrm{c}} \frac{\partial^{2} \widetilde{B}_{\mathrm{p}}}{\partial r^{2}}\right|_{\text {core }} ^{r_{\mathrm{i}} / r_{\mathrm{o}}}+\left.\eta_{\mathrm{ic} / \mathrm{m}} \frac{\partial^{2} \widetilde{B}_{\mathrm{p}}}{\partial r^{2}}\right|_{\mathrm{ic} / \mathrm{m}} ^{r_{\mathrm{i}} / r_{\mathrm{o}}}=0 \\
-\left.\eta_{\mathrm{c}} \frac{\partial}{\partial r} \frac{\partial \widetilde{B}_{\mathrm{t}}}{\partial \theta}\right|_{\text {core }} ^{r_{\mathrm{i}} / r_{\mathrm{o}}}+\left.\eta_{\mathrm{ic} / \mathrm{m}} \frac{\partial}{\partial r} \frac{\partial \widetilde{B}_{\mathrm{t}}}{\partial \theta}\right|_{\mathrm{ic} / \mathrm{m}} ^{r_{\mathrm{i}} / r_{\mathrm{o}}}=\left.r_{\mathrm{i} / \mathrm{o}} B_{\mathrm{r}}^{0}\left(\Omega_{\mathrm{ficb} / \mathrm{fcmb}}-\Omega_{\mathrm{ic} / \mathrm{m}}\right)\right|_{\mathrm{ic} / \mathrm{m}} ^{r_{\mathrm{i}} / r_{\mathrm{o}}} .
\end{aligned}
$$

In the following sections, we will therefore focus on the toroidal part of the radial derivative of $\widetilde{B}_{\varphi}$.

\subsubsection{Mantle-side}

We here formalize the relationship between the radial derivatives of the toroidal field on either sides of the CMB and the exact value at the CMB. In the case where the conductance of the mantle is embodied by a thin layer of constant electrical conductivity, the diffusion of the toroidal magnetic field is defined by equation (5.39). For each degree and order of the spherical harmonic expansion we have

$$
\left.r \widetilde{B}_{\mathrm{t}_{\ell}}^{m}(r)\right|_{\text {mantle }}=r_{\mathrm{o}} \widetilde{B}_{\mathrm{t}_{\ell}}^{m}\left(r_{\mathrm{o}}\right) \frac{\sinh \left[\left(\frac{1-i}{\delta_{\mathrm{m}}}\right)\left(r_{\mathrm{o}}+\Delta-r\right)\right]}{\sinh \left[\left(\frac{1-i}{\delta_{\mathrm{m}}}\right) \Delta\right]},
$$

that can be introduced in equation (5.27) in order to obtain

$$
\left.\widetilde{B}_{\mathrm{t}}\right|_{\text {mantle }}=r_{\mathrm{o}} \frac{\sinh \left[\left(\frac{1-i}{\delta_{\mathrm{m}}}\right)\left(r_{\mathrm{o}}+\Delta-r\right)\right]}{\sinh \left[\left(\frac{1-i}{\delta_{\mathrm{m}}}\right) \Delta\right]} \sum_{\ell, m}^{\mathrm{L}, \mathrm{M}} \widetilde{B}_{\mathrm{t}_{\ell}}^{m}\left(r_{\mathrm{o}}\right) Y_{\ell}^{m}(\theta, \varphi),
$$

in which we reduced the summation over the degree and order of the spherical harmonic expansion to $\sum_{\ell, m}^{\mathrm{L}, \mathrm{M}}$, in order to lighten the notation. The consecutive latitudinal and radial derivation (see A.2) gives, in $r=r_{\mathrm{o}}$,

$$
\left.\frac{\partial}{\partial r} \frac{\partial \widetilde{B}_{\mathrm{t}}}{\partial \theta}\right|_{\text {mantle }} ^{r_{\mathrm{o}}}=-\left(\frac{1-i}{\delta_{\mathrm{m}}}\right) r_{\mathrm{o}} \operatorname{coth}\left[\left(\frac{1-i}{\delta_{\mathrm{m}}}\right) \Delta\right] \times \frac{\partial}{\partial \theta}\left(\sum_{\ell, m}^{\mathrm{L}, \mathrm{M}} \widetilde{B}_{\mathrm{t}_{\ell}}^{m}\left(r_{\mathrm{o}}\right) Y_{\ell}^{m}(\theta, \varphi)\right) .
$$

At the $\mathrm{CMB}$, the value of the toroidal field is as defined by the boundary condition on the solution of $\widetilde{B}_{\mathrm{t}_{\ell}}^{m}$,

$$
\left.\frac{\partial \widetilde{B}_{\mathrm{t}}}{\partial \theta}\right|_{\text {mantle }} ^{r_{\mathrm{o}}}=r_{\mathrm{o}} \times \frac{\partial}{\partial \theta}\left(\sum_{\ell, m}^{\mathrm{L}, \mathrm{M}} \widetilde{B}_{\mathrm{t}_{\ell}}^{m}\left(r_{\mathrm{o}}\right) Y_{\ell}^{m}(\theta, \varphi)\right)
$$


that naturally leads to

$$
\left.\frac{\partial}{\partial r} \frac{\partial \widetilde{B}_{\mathrm{t}}}{\partial \theta}\right|_{\text {mantle }} ^{r_{\mathrm{o}}}=-\left(\frac{1-i}{\delta_{\mathrm{m}}}\right) \operatorname{coth}\left[\left(\frac{1-i}{\delta_{\mathrm{m}}}\right) \Delta\right] \times\left.\frac{\partial \widetilde{B}_{\mathrm{t}}}{\partial \theta}\right|_{\text {mantle }} ^{r_{\mathrm{o}}}
$$

Keeping in mind the continuity of the poloidal field and its first and second radial derivatives, this last equation means that

$$
\left.\frac{\partial \widetilde{B}_{\varphi}}{\partial r}\right|_{\text {mantle }} ^{r_{\mathrm{o}}}=-\widetilde{B}_{\varphi} \times\left.\left(\frac{1-i}{\delta_{\mathrm{m}}}\right) \operatorname{coth}\left[\left(\frac{1-i}{\delta_{\mathrm{m}}}\right) \Delta\right]\right|_{\text {mantle }} ^{r_{\mathrm{o}}}
$$

This equation formulates the link between the azimuthal field at the CMB and its radial derivative on the mantle side. In order to obtain a complete expression of $\widetilde{B}_{\varphi}$ at the CMB we now need to apply the same procedure on the fluid core side of the core-mantle boundary. On the core side, we have

$$
\left.\frac{\partial}{\partial r} \frac{\partial \widetilde{B}_{\mathrm{t}}}{\partial \theta}\right|_{\text {core }} ^{r_{\mathrm{o}}}=\left(\frac{1-i}{\delta_{\mathrm{c}}}\right) r_{\mathrm{o}} \times \frac{\partial}{\partial \theta}\left(\sum_{\ell, m}^{\mathrm{L}, \mathrm{M}} \widetilde{B}_{\mathrm{t}_{\ell}}^{m}\left(r_{\mathrm{o}}\right) Y_{\ell}^{m}(\theta, \varphi)\right),
$$

and thus, in $r=r_{\mathrm{o}}$

$$
\left.\frac{\partial}{\partial r} \frac{\partial \widetilde{B}_{\mathrm{t}}}{\partial \theta}\right|_{\text {core }} ^{r_{\mathrm{o}}}=\left(\frac{1-i}{\delta_{\mathrm{c}}}\right) r_{\mathrm{o}} \times \frac{\partial}{\partial \theta}\left(\sum_{\ell, m}^{\mathrm{L}, \mathrm{M}} \widetilde{B}_{\mathrm{t}_{\ell}}^{m}\left(r_{\mathrm{o}}\right) Y_{\ell}^{m}(\theta, \varphi)\right) .
$$

This expression also illustrates the link between the radial derivative of the azimuthal magnetic field in the core and its value at the CMB, as

and ultimately,

$$
\left.\frac{\partial}{\partial r} \frac{\partial \widetilde{B}_{\mathrm{t}}}{\partial \theta}\right|_{\text {core }} ^{r_{\mathrm{o}}}=\left(\frac{1-i}{\delta_{\mathrm{c}}}\right) \times\left.\frac{\partial B_{\mathrm{t}}}{\partial \theta}\right|_{\text {core }} ^{r_{\mathrm{o}}}
$$

$$
\left.\frac{\partial \widetilde{B}_{\varphi}}{\partial r}\right|_{\text {core }} ^{r_{\mathrm{o}}}=\widetilde{B}_{\varphi} \times\left.\left(\frac{1-i}{\delta_{\mathrm{c}}}\right)\right|_{\text {core }} ^{r_{\mathrm{o}}}
$$

Introducing the two radial derivatives of the azimuthal field, equations (5.72) and (5.76), into the conservation of the tangential electric field at the CMB (Eq. 5.59) gives

$$
\begin{aligned}
\eta_{\mathrm{c}} \widetilde{B}_{\varphi} \times\left.\left(\frac{1-i}{\delta_{\mathrm{c}}}\right)\right|_{\text {core }} ^{r_{\mathrm{o}}}+\eta_{\mathrm{m}} \widetilde{B}_{\varphi} \times\left(\frac{1-i}{\delta_{\mathrm{m}}}\right) \operatorname{coth}\left[\left(\frac{1-i}{\delta_{\mathrm{m}}}\right) \Delta\right] & \left.\right|_{\text {mantle }} ^{r_{\mathrm{o}}} \\
& =r_{\mathrm{o}} B_{\mathrm{r}}^{0}\left(\Omega_{\mathrm{fcmb}}-\Omega_{\mathrm{m}}\right)
\end{aligned}
$$

Since the magnetic field is continuous across the interface,

$$
\left.\widetilde{B}_{\varphi}\right|_{\text {core }} ^{r_{\mathrm{o}}}=\left.\widetilde{B}_{\varphi}\right|_{\text {mantle }} ^{r_{\mathrm{o}}}=\widetilde{B}_{\varphi}\left(r_{\mathrm{o}}\right),
$$


the solution becomes

$$
(1-i)\left\{\frac{\eta_{\mathrm{c}}}{\delta_{\mathrm{c}}}+\frac{\eta_{\mathrm{m}}}{\delta_{\mathrm{m}}} \operatorname{coth}\left[\left(\frac{1-i}{\delta_{\mathrm{m}}}\right) \Delta\right]\right\} \widetilde{B}_{\varphi}\left(r_{\mathrm{o}}\right)=r_{\mathrm{o}} B_{\mathrm{r}}^{0}\left(\Omega_{\mathrm{fcmb}}-\Omega_{\mathrm{m}}\right)
$$

and finally,

$$
\widetilde{B}_{\varphi}\left(r_{\mathrm{o}}\right)=\left(\frac{1+i}{2}\right) \frac{r_{\mathrm{o}} B_{\mathrm{r}}^{0}\left(\Omega_{\mathrm{fcmb}}-\Omega_{\mathrm{m}}\right)}{\frac{\eta_{\mathrm{c}}}{\delta_{\mathrm{c}}}+\frac{\eta_{\mathrm{m}}}{\delta_{\mathrm{m}}} \operatorname{coth}\left[\left(\frac{1-i}{\delta_{\mathrm{m}}}\right) \Delta\right]} .
$$

Equation (5.80) can also be expressed as,

$$
\widetilde{B}_{\varphi}\left(r_{\mathrm{o}}\right)=\Phi_{\mathrm{m}}\left(\delta_{\mathrm{c}}, \eta_{\mathrm{m}}, \Delta\right) r_{\mathrm{o}} B_{\mathrm{r}}^{0}\left(\Omega_{\mathrm{fcmb}}-\Omega_{\mathrm{m}}\right)
$$

with

$$
\Phi_{\mathrm{m}}\left(\delta_{\mathrm{c}}, \delta_{\mathrm{m}}, \Delta\right)=\left(\frac{1+i}{2}\right)\left\{\frac{\eta_{\mathrm{c}}}{\delta_{\mathrm{c}}}+\frac{\eta_{\mathrm{m}}}{\delta_{\mathrm{m}}} \operatorname{coth}\left[\left(\frac{1-i}{\delta_{\mathrm{m}}}\right) \Delta\right]\right\}^{-1},
$$

the transfer function between the velocity jump at the core-mantle boundary and the azimuthal part of the perturbed magnetic field produced, in $r=r_{\mathrm{o}}$.

\subsubsection{Inner core side}

At the inner core boundary, the same demonstration can be developed considering the solution for the diffusion of the toroidal field into the solid inner core,

$$
\left.\widetilde{B}_{\mathrm{t}}\right|_{\text {inner core }}=r e^{\left(\frac{1-i}{\delta_{\mathrm{c}}}\right)\left(r-r_{\mathrm{i}}\right)} \sum_{\ell, m}^{\mathrm{L}, \mathrm{M}} \widetilde{B}_{\mathrm{t}_{\ell}}^{m}\left(r_{\mathrm{i}}\right) Y_{\ell}^{m}(\theta, \varphi),
$$

and in fluid outer core,

$$
\left.\widetilde{B}_{\mathrm{t}}\right|_{\text {core }}=r e^{-\left(\frac{1-i}{\delta_{\mathrm{c}}}\right)\left(r-r_{\mathrm{i}}\right)} \sum_{\ell, m}^{\mathrm{L}, \mathrm{M}} \widetilde{B}_{\mathrm{t}_{\ell}}^{m}\left(r_{\mathrm{i}}\right) Y_{\ell}^{m}(\theta, \varphi) .
$$

Taking the latitudinal and radial derivative of equation (5.83) gives

$$
\left.\frac{\partial}{\partial r} \frac{\partial \widetilde{B}_{\mathrm{t}}}{\partial \theta}\right|_{\text {inner core }}=r\left(\frac{1-i}{\delta_{\mathrm{c}}}\right) \frac{\partial}{\partial \theta}\left(\sum_{\ell, m}^{\mathrm{L}, \mathrm{M}} \widetilde{B}_{\mathrm{t}_{\ell}}^{m}\left(r_{\mathrm{i}}\right) Y_{\ell}^{m}(\theta, \varphi)\right)
$$

that, at $r=r_{\mathrm{i}}$, is similar to

$$
\left.\frac{\partial}{\partial r} \frac{\partial \widetilde{B}_{\mathrm{t}}}{\partial \theta}\right|_{\text {inner core }} ^{r_{\mathrm{i}}}=r_{\mathrm{i}}\left(\frac{1-i}{\delta_{\mathrm{c}}}\right) \frac{\partial}{\partial \theta}\left(\sum_{\ell, m}^{\mathrm{L}, \mathrm{M}} \widetilde{B}_{\mathrm{t}_{\ell}}^{m}\left(r_{\mathrm{i}}\right) Y_{\ell}^{m}(\theta, \varphi)\right) .
$$


In the fluid core we also have,

$$
\left.\frac{\partial}{\partial r} \frac{\partial \widetilde{B}_{\mathrm{t}}}{\partial \theta}\right|_{\text {core }}=-r\left(\frac{1-i}{\delta_{\mathrm{c}}}\right) e^{-\left(\frac{1-i}{\delta_{\mathrm{c}}}\right)\left(r-r_{\mathrm{i}}\right)} \frac{\partial}{\partial \theta}\left(\sum_{\ell, m}^{\mathrm{L}, \mathrm{M}} \widetilde{B}_{\mathrm{t}_{\ell}}^{m}\left(r_{\mathrm{i}}\right) Y_{\ell}^{m}(\theta, \varphi)\right),
$$

which evaluation at the ICB gives

$$
\left.\frac{\partial}{\partial r} \frac{\partial \widetilde{B}_{\mathrm{t}}}{\partial \theta}\right|_{\text {core }} ^{r_{\mathrm{i}}}=-r_{\mathrm{i}}\left(\frac{1-i}{\delta_{\mathrm{c}}}\right) \frac{\partial}{\partial \theta}\left(\sum_{\ell, m}^{\mathrm{L}, \mathrm{M}} \widetilde{B}_{\mathrm{t}_{\ell}}^{m}\left(r_{\mathrm{i}}\right) Y_{\ell}^{m}(\theta, \varphi)\right) .
$$

Relating equations (5.86) and (5.88) to their respective values at the ICB leads to

and

$$
\left.\frac{\partial}{\partial r} \frac{\partial \widetilde{B}_{\mathrm{t}}}{\partial \theta}\right|_{\text {inner core }} ^{r_{\mathrm{i}}}=\left.\left(\frac{1-i}{\delta_{\mathrm{c}}}\right) \frac{\partial \widetilde{B}_{\mathrm{t}}}{\partial \theta}\right|_{\text {inner core }} ^{r_{\mathrm{i}}},
$$

$$
\left.\frac{\partial}{\partial r} \frac{\partial \widetilde{B}_{\mathrm{t}}}{\partial \theta}\right|_{\text {core }} ^{r_{\mathrm{i}}}=-\left.\left(\frac{1-i}{\delta_{\mathrm{c}}}\right) \frac{\partial \widetilde{B}_{\mathrm{t}}}{\partial \theta}\right|_{\text {core }} ^{r_{\mathrm{i}}} .
$$

Keeping in mind the continuity of the second radial derivative of the poloidal field, we have

and

$$
\left.\frac{\partial \widetilde{B}_{\varphi}}{\partial r}\right|_{\text {inner core }} ^{r_{\mathrm{i}}}=\left.\widetilde{B}_{\varphi}\left(\frac{1-i}{\delta_{\mathrm{c}}}\right)\right|_{\text {inner core }} ^{r_{\mathrm{i}}},
$$

$$
\left.\frac{\partial \widetilde{B}_{\varphi}}{\partial r}\right|_{\text {core }} ^{r_{\mathrm{i}}}=-\left.\widetilde{B}_{\varphi}\left(\frac{1-i}{\delta_{\mathrm{c}}}\right)\right|_{\text {core }} ^{r_{\mathrm{i}}}
$$

Introducing these two radial derivatives into the conservation of the tangential electric field at the ICB gives

$$
\left.\widetilde{B}_{\varphi} \eta_{\mathrm{c}}\left(\frac{1-i}{\delta_{\mathrm{c}}}\right)\right|_{\text {core }} ^{r_{\mathrm{i}}}+\left.\widetilde{B}_{\varphi} \eta_{\mathrm{c}}\left(\frac{1-i}{\delta_{\mathrm{c}}}\right)\right|_{\text {inner core }} ^{r_{\mathrm{i}}}=r_{\mathrm{i}} B_{\mathrm{r}}^{0}\left(\Omega_{\mathrm{ficb}}-\Omega_{\mathrm{ic}}\right)
$$

As the perturbed magnetic field $\widetilde{\mathbf{B}}_{1}$ is continuous across the ICB,

$$
\left.\widetilde{B}_{\varphi}\right|_{\text {core }} ^{r_{\mathrm{i}}}=\left.\widetilde{B}_{\varphi}\right|_{\text {inner core }} ^{r_{\mathrm{i}}}=\widetilde{B}_{\varphi}\left(r_{\mathrm{i}}\right)
$$

we have,

$$
2 \eta_{\mathrm{c}}\left(\frac{1-i}{\delta_{\mathrm{c}}}\right) \widetilde{B}_{\varphi}\left(r_{\mathrm{i}}\right)=r_{\mathrm{i}} B_{\mathrm{r}}^{0}\left(\Omega_{\mathrm{ficb}}-\Omega_{\mathrm{ic}}\right)
$$

and ultimately,

$$
\widetilde{B}_{\varphi}\left(r_{\mathrm{i}}\right)=\left(\frac{1+i}{4}\right)\left(\frac{\delta_{\mathrm{c}}}{\eta_{\mathrm{c}}}\right) r_{\mathrm{i}} B_{\mathrm{r}}^{0}\left(\Omega_{\mathrm{ficb}}-\Omega_{\mathrm{ic}}\right) .
$$


Like at the core-mantle boundary, we can represent this solution defining a transfer function $\Phi_{\text {ic }}$ between the velocity jump at the inner core boundary and the azimuthal part of the perturbed magnetic field produced as,

$$
\widetilde{B}_{\varphi}\left(r_{\mathrm{i}}\right)=\Phi_{\mathrm{ic}}\left(\delta_{\mathrm{c}}\right) r_{\mathrm{i}} B_{\mathrm{r}}^{0}\left(\Omega_{\mathrm{ficb}}-\Omega_{\mathrm{ic}}\right)
$$

with

$$
\Phi_{\mathrm{ic}}\left(\delta_{\mathrm{c}}\right)=\left(\frac{1+i}{4}\right) \frac{\delta_{\mathrm{c}}}{\eta_{\mathrm{c}}}
$$

\subsubsection{Discussion on the solutions of $\widetilde{B}_{\varphi}$ at ICB and CMB}

Figure 5.7 illustrates the phase lag between the velocity jumps at ICB and CMB and the perturbed magnetic field produced, as expressed by equations (5.97) and (5.81). This phase lag is determined by

$$
\arctan \left(\frac{\mathcal{I} m(\Phi)}{\mathcal{R} e(\Phi)}\right)
$$

with $\mathcal{I} m(\Phi)$ the imaginary part and $\mathcal{R} e(\Phi)$ the real part of the transfer function between the velocity jump and the perturbed azimuthal field at either $\operatorname{ICB}\left(\Phi_{\mathrm{ic}}\right)$ or $\operatorname{CMB}\left(\Phi_{\mathrm{m}}\right)$.

At the inner core boundary, this phase lag is equal to $\frac{\pi}{4}$ though this value may no longer be relevant for signal periods on the order of the magnetic diffusion timescale. Indeed, the above demonstration is contingent upon

$$
\omega \tau_{\eta} \gg 1
$$

which is a "high-frequency" approximation over geological timescales.

At the core-mantle boundary, the form of the transfer function $\Phi_{\mathrm{m}}$ is mainly determined by $\Delta$, the thickness of the layer. For a very thin layer of 150 meters with the electrical conductivity of the core (blue line figure 5.7), there is no phase lag between the velocity jump and the perturbed magnetic field produced for $\omega \tau_{\eta}<5.7 \times 10^{6}$, that is for periods higher than 4 months, to the left of figure 5.7. In this domain, the thickness of the layer $\Delta$ is small compared to the skin depth $\delta_{\mathrm{c}}$, causing the hyperbolic cotangent term to tend to infinity and the phase lag to be zero, since

$$
\left\{\frac{\eta_{\mathrm{c}}}{\delta_{\mathrm{c}}}+\frac{\eta_{\mathrm{m}}}{\delta_{\mathrm{m}}} \operatorname{coth}\left[\left(\frac{1-i}{\delta_{\mathrm{m}}}\right) \Delta\right]\right\}^{-1} \approx(1-i) \frac{\Delta}{\eta_{\mathrm{m}}} .
$$

In this case, the transfer function at the CMB simply becomes a function of the thickness and the magnetic diffusivity of the electrically conducting layer,

$$
\Phi_{\mathrm{m}}(\omega)=\frac{\Delta}{\eta_{\mathrm{m}}} .
$$




\section{Phase lag}

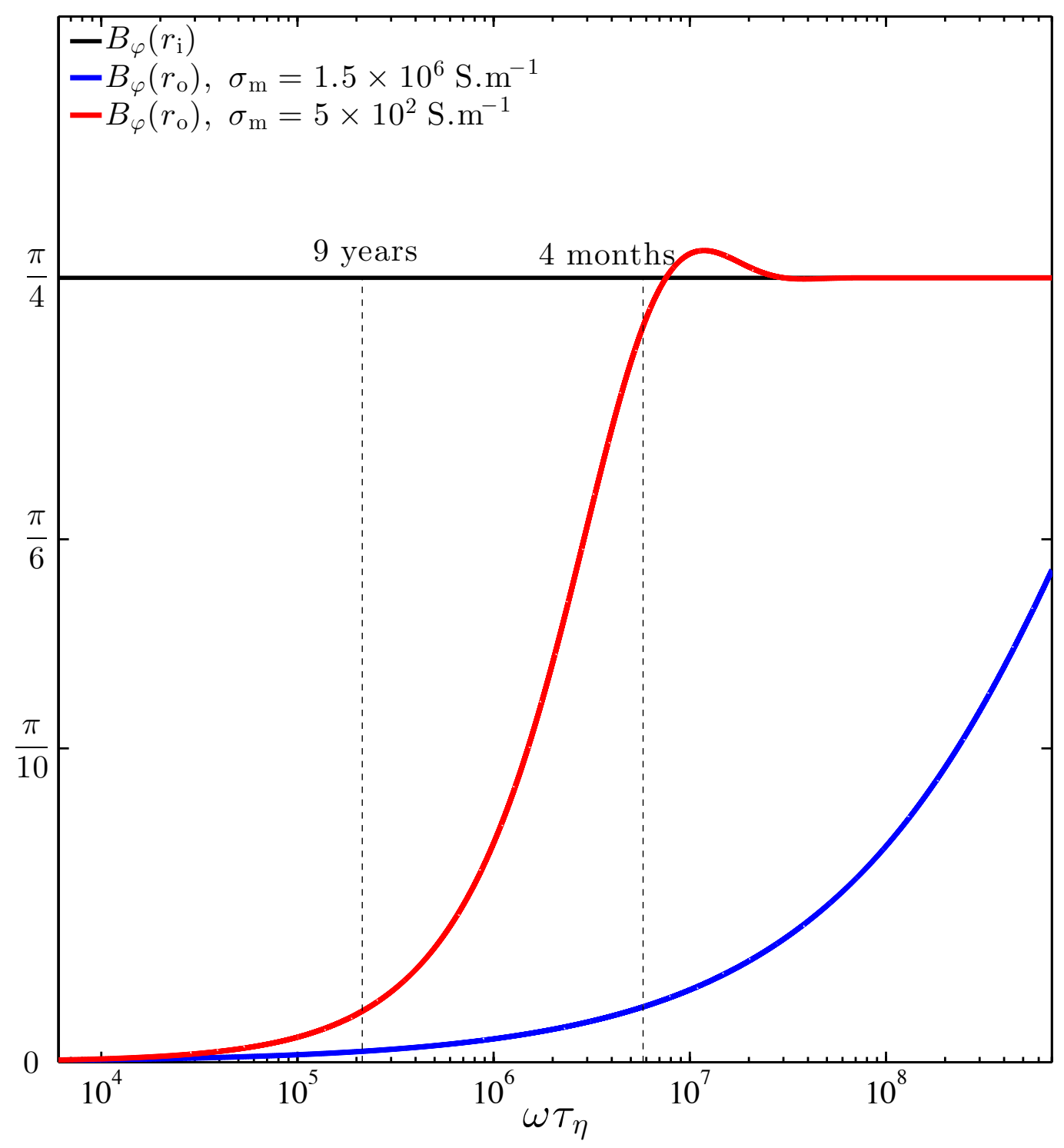

Figure 5.7: Phase lag between the velocity jump at the ICB and the CMB and the azimuthal part of the perturbed magnetic field $\widetilde{\mathbf{B}}_{1}$ at the ICB in black (Eq. 5.97), at the CMB in blue and red

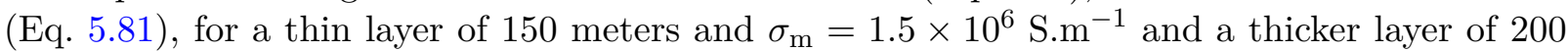
kilometers and $\sigma_{\mathrm{m}}=5 \times 10^{2} \mathrm{~S} . \mathrm{m}^{-1}$, respectively. The vertical dashed-black lines correspond to two limits below which the phase lag may be considered at negligible at the CMB. The left one stands for the very thin layer case for which the phase lag is negligible for periods greater than 9 years. The right one represents the same limit of a thicker conducting laye for wich the phase lag become significant for periods lower than 4 months. 
For signals with periods shorter than 4 months, the skin depth $\delta_{\mathrm{m}}$ progressively approaches the thickness of the layer, and as the period shortens the phase lag increases. For a thicker layer (in red figure 5.7), the increase of the phase lag appears for $\omega \tau_{\eta}>2 \times 10^{5}$, corresponding to periods shorter than 9 years. In that case, as the signal period is shorter and shorter, the solution progressively recovers the limit defined at the ICB, where the skin depth becomes shorter than the thickness of the layer (i.e. $\delta_{\mathrm{m}} \ll \Delta$ ) and the phase equal to $\frac{\pi}{4}$.

Figure 5.7 represents the limits of "secular variation domain", the interval in which the phase lag between the velocity jumps at ICB and CMB and the perturbed magnetic field are $\frac{\pi}{4}$ and zero, respectively. This domain is delimited by signal periods greater than 4 months, where the skin depth of the perturbed diffusing into the lower mantle $\delta_{\mathrm{m}}$ is significantly larger than the thickness of the layer $(\Delta=150 \mathrm{~m})$. Moreover, in that range of signal periods, the transfer functions between the velocity jumps and the azimuthal field can be simply expressed as functions of the angular frequency of the signal $\omega$, the magnetic diffusivities of the core and the conducting part of the mantle, $\eta_{\mathrm{c}}$ and $\eta_{\mathrm{m}}$, and the thickness of the conducting layer $\Delta$. If the conductance of the lower mantle is embodied by a layer of 200 kilometers with an electrical conductivity $\sigma_{\mathrm{m}}=1.5 \times 10^{2} \mathrm{~S}_{\mathrm{m}} \mathrm{m}^{-1}$, the "secular variation domain" is restricted to periods grater than 9 years.

\subsection{Electromagnetic torque models}

The previous sections provide the transfer functions between the velocity jumps at the fluid core boundaries and the perturbation of the azimuthal magnetic field produced. In order to describe the time-dependent electromagnetic torque at either ICB and CMB, the next step is to introduce the expression of (5.81) and (5.97) into the perturbation of the electromagnetic torque that we recall are defined as

$$
\begin{aligned}
\widetilde{\Gamma}_{\mathrm{ICB}}^{1}(\omega) & =\frac{r_{\mathrm{i}}}{\mu_{0}} \int_{S_{\mathrm{ICB}}} B_{\mathrm{r}}^{0} \widetilde{B}_{\varphi}(\omega) \sin \theta \mathrm{dS}, \quad \text { and } \\
\widetilde{\Gamma}_{\mathrm{CMB}}^{1}(\omega) & =-\frac{r_{\mathrm{o}}}{\mu_{0}} \int_{S_{\mathrm{CMB}}} B_{\mathrm{r}}^{0} \widetilde{B}_{\varphi}(\omega) \sin \theta \mathrm{dS} .
\end{aligned}
$$

At the core-mantle boundary, introducing the simple expression of $\Phi_{\mathrm{m}}$ (Eq. 5.102) into equation (5.81) leads to

$$
\widetilde{B}_{\varphi}=\frac{\Delta}{\eta_{\mathrm{m}}} B_{\mathrm{r}}^{0} r_{\mathrm{o}}\left(\Omega_{\mathrm{fcmb}}-\Omega_{\mathrm{m}}\right)
$$

which may then be introduced into equation (5.104), leading to

or even

$$
\widetilde{\Gamma}_{\mathrm{CMB}}^{1}(\omega)=-\frac{r_{\mathrm{o}}}{\mu_{0}} \int_{S_{\mathrm{CMB}}}\left(B_{\mathrm{r}}^{0}\right)^{2} \frac{\Delta}{\eta_{\mathrm{m}}} r_{\mathrm{o}}\left(\Omega_{\mathrm{fcmb}}-\Omega_{\mathrm{m}}\right) \sin \theta \mathrm{dS}
$$

$$
\widetilde{\Gamma}_{\mathrm{CMB}}^{1}(\omega)=-r_{\mathrm{o}}^{2} \sigma_{\mathrm{m}} \Delta\left(\Omega_{\mathrm{fcmb}}-\Omega_{\mathrm{m}}\right) \int_{S_{\mathrm{CMB}}}\left(B_{\mathrm{r}}^{0}\right)^{2} \sin \theta \mathrm{dS},
$$


Finally, as the surface integral of the radial component of the main field corresponds to a mean squared value of the radial field at the CMB, it will be simply noted like $B_{r_{\mathrm{o}}}^{2}$, and we have

$$
\widetilde{\Gamma}_{\mathrm{CMB}}^{1}(\omega)=-r_{\mathrm{o}}^{4} \sigma_{\mathrm{m}} \Delta B_{r_{\mathrm{o}}}^{2}\left(\Omega_{\mathrm{fcmb}}-\Omega_{\mathrm{m}}\right) \text {. }
$$

The same analysis at the inner core boundary leads to

$$
\widetilde{\Gamma}_{\mathrm{ICB}}^{1}(\omega)=\frac{r_{\mathrm{i}}^{4}}{\mu_{0}} B_{r_{\mathrm{i}}}^{2} \Phi_{\mathrm{ic}}\left(\delta_{\mathrm{c}}\right)\left(\Omega_{\mathrm{ficb}}-\Omega_{\mathrm{ic}}\right)
$$

in the general case and

$$
\widetilde{\Gamma}_{\mathrm{ICB}}^{1}(\omega)=\frac{r_{\mathrm{i}}^{4}}{\mu_{0}} B_{r_{\mathrm{i}}}^{2}\left(\frac{1+i}{4}\right) \sqrt{\frac{2}{\eta_{\mathrm{c}} \omega}}\left(\Omega_{\mathrm{ficb}}-\Omega_{\mathrm{ic}}\right),
$$

or even

$$
\widetilde{\Gamma}_{\mathrm{ICB}}^{1}(\omega)=\left(\frac{1+i}{4}\right) r_{\mathrm{i}}^{4} B_{r_{\mathrm{i}}}^{2} \sqrt{\frac{2 \sigma_{\mathrm{c}}}{\mu_{0} \omega}}\left(\Omega_{\mathrm{ficb}}-\Omega_{\mathrm{ic}}\right),
$$

for the "extended secular variation domain" defined in figure 5.7, that is to say for signal periods between 3400 years and 4 months.

Equations (5.108) and (5.111) illustrated the time-dependent behavior of the electromagnetic torques at the fluid core boundaries. These expressions follow from a perturbation analysis in which the perturbed azimuthal field that characterizes the electromagnetic torques results from the shear of a mainly dipolar, slowly varying over time, magnetic field by the velocity jumps at the fluid core boundaries. As these models shall constitute the basis of the time-dependent rotational dynamics of the system, it is worth recalling which assumptions they rest upon. Firstly, the perturbations analysis itself and the extraction of the major source term of azimuthal field may be a source of uncertainties. Secondly, in order to obtain a homogeneous second order equation to describe the diffusion of the perturbed field away from the boundaries, we had to neglect the horizontal variations compared to the radial ones. Section 5.1.2 highlights the fact that this assumption is highly sensitive to the signal period and the thickness of the electrically conducting part of the mantle. The situation is even more arguable for the solution in the inner core as $r \mapsto 0$. However, the models are built by linking the radial derivatives of the azimuthal field "at" the fluid core boundaries, so this shall not be a major complication. Finally, we assumed that the perturbation of the azimuthal field is mainly carried by the toroidal field. This is strictly valid if the perturbation is induced by the shear of a mainly dipolar field by an axisymmetric velocity jump, ensuring the continuity of the second radial derivative of the poloidal part of the magnetic field. 


\title{
Elementary cases: validation of the electromagnetic torque models
}

\begin{abstract}
This chapter is dedicated to the validation of the time-dependent electromagnetic torque models acting on the mantle and the inner core as expressed in the previous chapter. This validation is performed on simple case-study, that is to say in non-convective numerical simulations in which the main magnetic field and velocity perturbations are imposed. We performed and analyzed the results of 52 case-study simulations; 32 with an oscillating solid body rotation in the outer core in order to focus on the influence of the velocity jumps on the system, and 20 simulations with a shear flow in the fluid outer core to examine the possible effects of a remote shear. For each core flow, we also consider the impact of a gravitational coupling linking the inner core to the mantle. This provides a first approach to the time-dependent behavior of the system and allows the verification of the electromagnetic torque models under the assumptions formulated to obtain the expressions.
\end{abstract}

\section{Résumé}

Ce chapitre est consacré à la validation des modèles de couple électromagnétique dépendants du temps agissant sur le manteau et le noyau interne développés dans le chapitre précédent. Cette validation est effectuée sur des cas d'étude, c'est-à-dire dans des simulations numériques non convectives dans lesquelles le champ magnétique et les perturbations de vitesse sont imposés. Nous avons effectué et analysé les résultats de 52 simulations ; 32 avec une rotation solide oscillante dans le noyau externe afin de se concentrer sur l'influence des sauts de vitesse, et 20 simulations avec un écoulement cisaillant dans le noyau externe fluide pour examiner les possibles effets d'un cisaillement distant des frontières. Pour chaque écoulement, nous considérons également l'impact d'un couplage gravitationnel reliant le noyau interne au manteau. Ceci fournit une première approche du comportement dépendant du temps du système et permet la validation des modèles de couple électromagnétique dans le cadre des hypothèses formulées pour obtenir les expressions. 


\subsection{System}

The system studied consider two electromagnetic torques at the fluid core boundaries, $\Gamma_{\mathrm{ICB}}$ and $\Gamma_{\mathrm{CMB}}$, and a gravitational torque, $\Gamma_{\mathrm{G}}$, that links the inner core and the mantle. The conservation of the angular momentum of the inner core, the mantle, and the fluid outer core is then

$$
\begin{aligned}
\mathrm{I}_{\mathrm{i}} \frac{\mathrm{d} \Omega_{\mathrm{ic}}}{\mathrm{dt}} & =\Gamma_{\mathrm{G}}+\Gamma_{\mathrm{ICB}} \\
\mathrm{I}_{\mathrm{m}} \frac{\mathrm{d} \Omega_{\mathrm{m}}}{\mathrm{dt}} & =-\Gamma_{\mathrm{G}}+\Gamma_{\mathrm{ICB}} \\
\frac{\mathrm{d}}{\mathrm{dt}} \int_{V_{f}} \omega_{\mathrm{f}} \mathrm{i}_{\mathrm{f}} \mathrm{d} V & =-\Gamma_{\mathrm{ICB}}-\Gamma_{\mathrm{CMB}}
\end{aligned}
$$

with $\Omega_{\mathrm{ic}}$ and $\Omega_{\mathrm{m}}$ the angular velocities and $\mathrm{I}_{\mathrm{i}}$ and $\mathrm{I}_{\mathrm{m}}$ the moments of inertia of the inner core and the mantle, $V_{f}$ the volume of the outer core, and $\omega_{\mathrm{f}}$ and $\mathrm{i}_{\mathrm{f}}$ respectively the angular velocity and the moment of inertia of a fluid parcel in the fluid outer core. This set of equation is naturally solved in the numerical implementation and defines the time-dependent responses of the inner core and the mantle to the different torques. The consistency check is then to predict the responses of the inner core and the mantle using the dynamical electromagnetic torque models. To to so, we will impose a periodic azimuthal flow into the fluid outer, that will interact with a static dipolar magnetic field of the form

$$
B_{\mathrm{p} 1}^{0}\left(r_{\mathrm{o}} \geq r \geq r_{\mathrm{i}}\right)=-B_{\mathrm{i}} \frac{5}{16 \sqrt{3}}\left(6 r^{2}-r_{\mathrm{o}} r+2 \frac{r_{\mathrm{i}}^{3}}{r}\right) \text {, }
$$

in the fluid outer core, and

$$
B_{\mathrm{p}_{1}}^{0}\left(r \leq r_{\mathrm{i}}\right)=\frac{r}{r_{\mathrm{i}}} B_{\mathrm{p}_{1}}^{0}\left(r_{\mathrm{i}}\right)
$$

in the inner core, with $B_{\mathrm{i}}$ a given amplitude of the poloidal field, as pictured by the black lines in figure 6.1. Note that the form of this poloidal magnetic field is commonly used to start convective simulations of the geodynamo, in accordance with the benchmark of Christensen et al. (2001). The loops solving the temperature, the poloidal and toroidal velocity fields as well as the poloidal magnetic field are muted, and we adopt stress-free boundary conditions at both ICB and CMB. Hence, the case-study simulations only compute the azimuthal field produced by the shear of a dipolar magnetic field by an imposed azimuthal velocity field. As a consequence, the amplitude of the main dipolar magnetic field and strength of the velocity perturbations should be the main control parameters. In that sense, we choose $\Omega_{\mathrm{oc}}$, the amplitude of the oscillating perturbation imposed to the outer core as a characteristic dimension for the angular velocities of the system. The characteristic dimension of the magnetic field is taken as $\mathrm{B}_{\mathrm{dip}}$, the strength of the dipole field at the core-mantle boundary; leading to electromagnetic torques scaling like $\frac{D^{3} \mathrm{~B}_{\mathrm{dip}}}{\mu_{0}}$. The thickness of the fluid outer core $D$ is also the characteristic length-scale, $\rho$ is the mean density of the core, $\eta_{\mathrm{c}}$ the magnetic diffusivity, $\mu_{0}$ the permeability of free space and the dimension of the time is given by 
$\tau_{\eta}=D^{2} / \eta_{\mathrm{c}}$. In order to lighten the notation, every variable and physical parameter will, from now, be displayed as dimensionless, so that we have

$$
\begin{array}{lll}
\Omega=\Omega / \Omega_{\mathrm{oc}}, & \omega=\omega \times \tau_{\eta}, & t=t / \tau_{\eta}, \\
\mathbf{B}=\mathbf{B} / \mathrm{B}_{\mathrm{dip}}, & \Gamma_{\mathrm{ICB} / \mathrm{CMB}}=\Gamma_{\mathrm{ICB} / \mathrm{CMB}} / \frac{D^{3} \mathrm{~B}_{\mathrm{dip}}^{2}}{\mu_{0}}, & \Gamma_{\mathrm{G}}=\Gamma_{\mathrm{G}} / \rho \eta_{\mathrm{c}}^{2} D .
\end{array}
$$

When using this characteristic dimensions, and for a given frequency of the velocity perturbation, the system can be fully described by two dimensionless numbers. The first one is

$$
\mathcal{P}=\frac{\eta}{\Omega_{\mathrm{oc}} D^{2}},
$$

and compares the amplitude of the perturbation relative to the magnetic diffusion time of the core, and the second is the Lundquist number,

$$
\mathcal{L} u=\frac{D B_{\text {dip }}}{\eta_{\mathrm{c}} \sqrt{\mu_{0} \rho}}
$$

that compares the time for an Alfvén wave to travel from ICB to CMB, to the magnetic diffusion time of the core $\tau_{\eta}$. Indeed, the Lundquist number may also be written

$$
\mathcal{L} u=\frac{D V_{\mathrm{A}}}{\eta_{\mathrm{c}}}=\frac{\tau_{\eta}}{t_{\mathrm{A}}}
$$

with $V_{\mathrm{A}}=\mathrm{B}_{\mathrm{dip}} / \sqrt{\mu_{0} \rho}$, the Alfvén wave velocity and $t_{\mathrm{A}}=D / V_{\mathrm{A}}$, the time for an Alfvén wave to cover a distance $D$. Please note that the Lundquist scaling of this system was already anticipated by Gubbins (1981). Indeed, even though we do not literally treat the problem of Alfvén waves in this chapter, the relevance of the Lundquist scaling lies in the fact that the main driving/restoring force in our system is the Lorentz force, as it is the case of the Alfvén waves.

\subsection{Solid-body rotation}

\subsubsection{Case-study simulations}

In order to study the responses of the inner core and the mantle to the velocity jumps at the fluid core boundaries, the first series of case-study simulations pictures a periodic solid-body rotation of the outer core, implemented as a toroidal velocity field of the form

$$
V_{\mathrm{t} 1}^{1}(r)=\Omega_{\mathrm{oc}} \sin (\omega t) \frac{r}{\sqrt{3}}
$$

In this particular situation, the outer core can be described by a moment of inertia $\mathrm{I}_{\mathrm{oc}}$ and an angular velocity of rotation $\Omega_{\mathrm{oc}}$, as pictured in figure 6.1. This figure also shows the form of the poloidal field implemented (Eq. 6.2), the responses of the inner core and the 
mantle (on the left) in term of angular velocities and the azimuthal field produced by the velocity jumps at the fluid core boundaries (on the right).
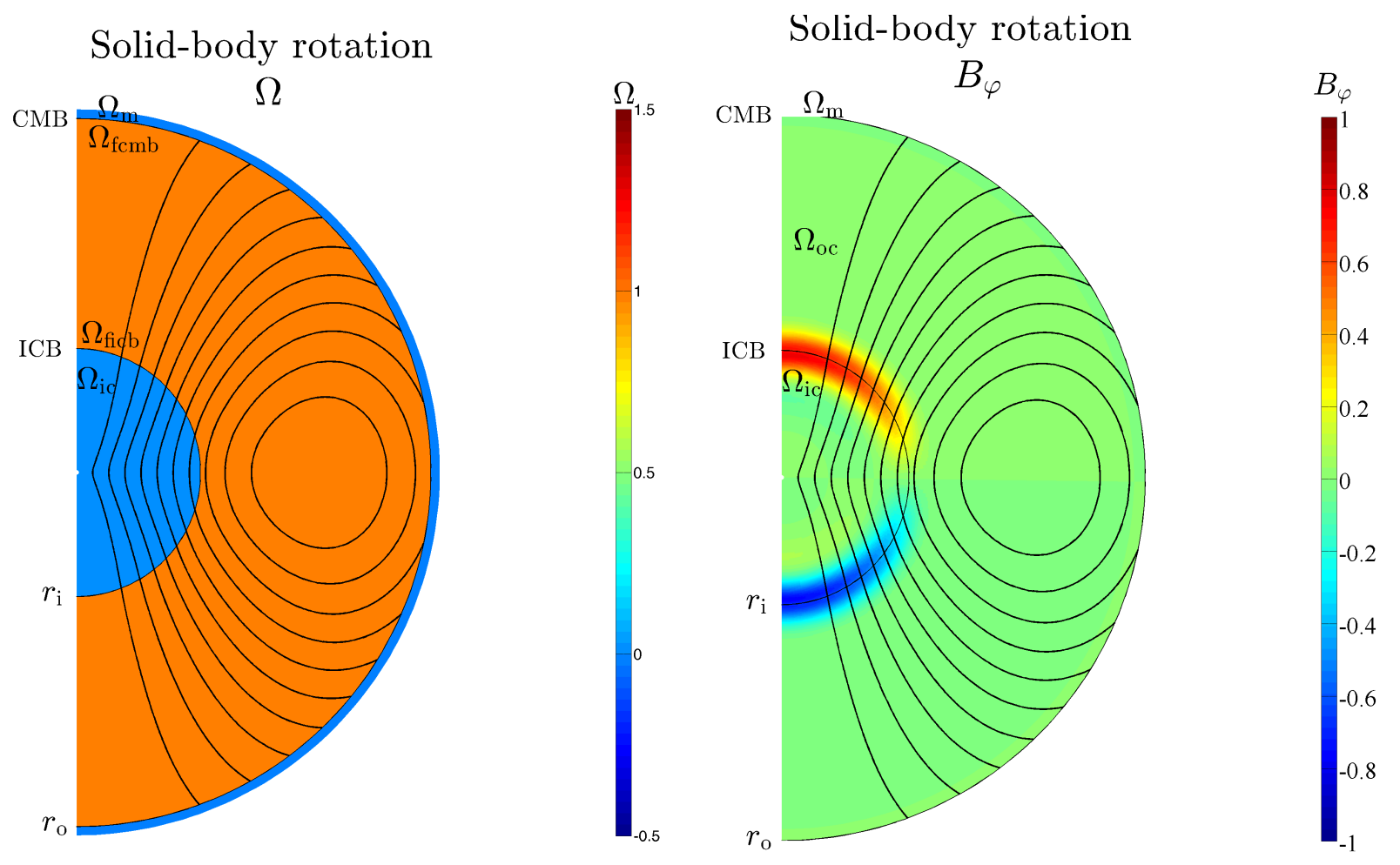

Figure 6.1: Snapshots of a case-study simulation with an imposed solid-body rotation in the outer core and a static poloidal magnetic field (black lines). On the left, the angular velocities of the inner core $\Omega_{\mathrm{ic}}$, the fluid outer core $\Omega_{\mathrm{oc}}$ and the mantle $\Omega_{\mathrm{m}}$. On the right, the azimuthal magnetic field produced by the velocity jumps at ICB and CMB.

In this part, we performed a set of 32 numerical simulations with dimensionless frequencies of periodic forcing $f$ ranging from 1 to 10000 with an amplitude $\Omega_{\mathrm{oc}}=1$ (Eq. 6.8). Given our choice of characteristic dimensions, the amplitude of the dipole $\mathrm{B}_{\text {dip }}$ is also one, $\mathcal{P}=8.3 \times 10^{-3}$ and the Lundquist number is $\mathcal{L} u=43$. Each simulation pictures a transient regime as illustrated by figure 6.2. Once the system reaches stationarity, we record the amplitude and the possible phase-lag between the different angular velocities of the system and the electromagnetic torques. Figure 6.2 presents an example of the evolution of the angular velocity of the inner core (in black) triggered by a periodic solid-body forcing of the outer core (in blue) with $f=200$, without gravitational coupling between the inner core and the mantle.

\subsubsection{Without gravitational coupling}

We here focus on the results of the 12 numerical case-study simulations without gravitational coupling linking the inner core to the mantle. In this particular setup, the electromagnetic torques at ICB and CMB should balance in the long-term state. However, this balance is not instantly fulfilled, essentially due to the difference between the moments of inertia of the mantle and the inner core. Moreover, as the moment of inertia of the 


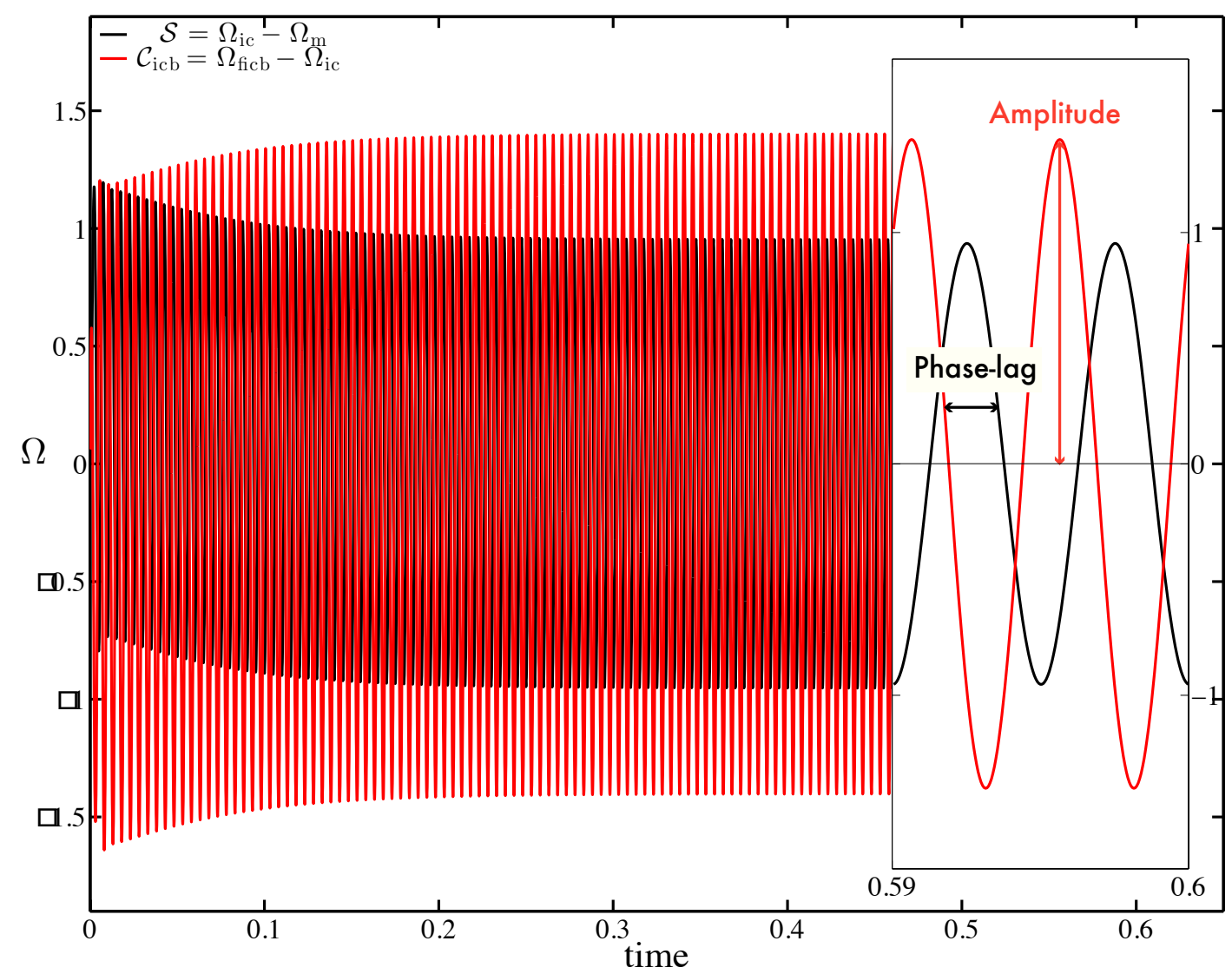

Figure 6.2: Inner core super-rotation $\mathcal{S}$ and shear at the ICB $\mathcal{C}_{\text {icb }}$, for a periodic forcing with frequency $f=200$. The left part shows the transient regime while the zoomed right part illustrates the monitoring of the amplitude and the phase-lag.

mantle is about a thousand times greater than the one of the inner core, an electromagnetic torque at the CMB should be a thousand times larger than at the ICB in order to obtain a consequent perturbation in the angular velocity of rotation of the mantle. Thus, when we require a periodic solid-body rotation in the outer core, with the same amplitude for each frequency, the shear at the CMB $\left(\Omega_{\mathrm{oc}}-\Omega_{\mathrm{m}}\right)$ remains unchanged. Consequently, it is ambitious to validate the model of the perturbed electromagnetic torque at the CMB with this approach, and the analysis of the case-study simulations focuses on the scaling of the electromagnetic torque acting on the inner core.

Figure 6.3 presents the results of the first series of numerical simulation, in terms of the amplitude of the electromagnetic torque at the ICB (in black) and the amplitude of the inner core response (in blue). For very low frequencies (periods close to the magnetic diffusion time of the core $\left.\tau_{\eta}\right), f \mapsto 1$, and equivalently, $\omega \mapsto 2 \pi$, the inner core and the outer core are rotating at the same angular frequency, i.e. $\Omega_{\mathrm{oc}}-\Omega_{\mathrm{ic}}=0$ over time. As the frequency of the outer core rotation is increased, the co-rotation regime is disrupted. Around angular frequencies of $\omega=10^{3}$, the amplitude of the electromagnetic torque at the ICB reaches a peak, that coincides with a peak of the angular velocity of the inner core. When the frequency is further increased, the rotation of the inner core decreases $\left(\Omega_{\mathrm{oc}}-\Omega_{\mathrm{ic}} \mapsto \Omega_{\mathrm{oc}}\right)$, along with the amplitude of $\widetilde{\Gamma}_{\mathrm{ICB}}^{1}$. 


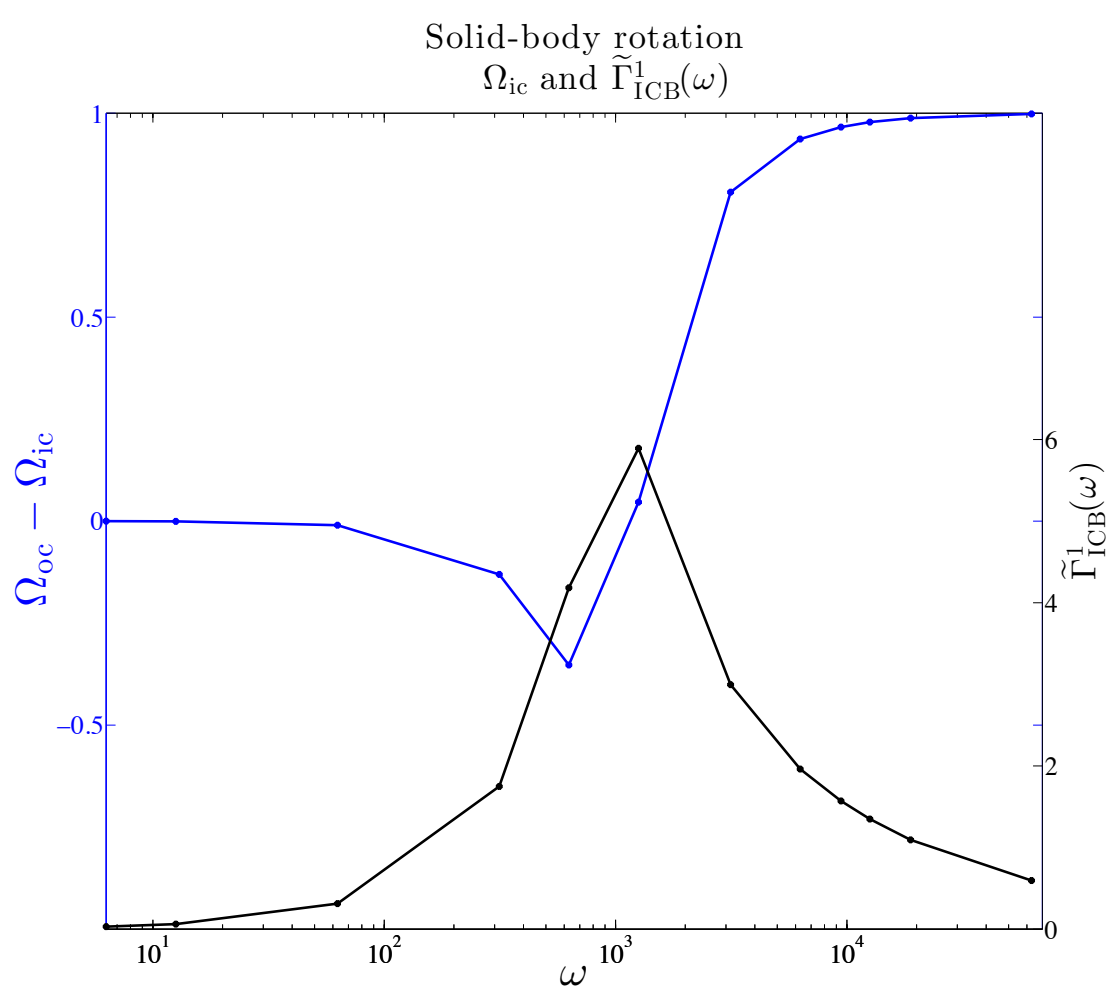

Figure 6.3: Amplitude of the angular velocity jump at the ICB, $\Omega_{\mathrm{oc}}-\Omega_{\mathrm{ic}}$, and amplitude of the electromagnetic torque exerted on the inner core $\widetilde{\Gamma}_{\mathrm{ICB}}^{1}$, as a function of the angular frequency $\omega$ of the periodic forcing.

Figure 6.4 shows the agreement between the model of the perturbed electromagnetic torque at the ICB developed in chapter 5 and the results obtained with a set of 12 numerical simulations in which the outer core is solid-body rotating at a given frequency. Since the lowest frequencies correspond to periods of the order of the magnetic diffusion time of the core, the scaling of $\widetilde{\Gamma}_{\mathrm{ICB}}^{1}$ is realized with the complete expression of the transfer function $\Phi_{\text {ic }}$ between the velocity jump and the azimuthal field produced at the ICB. The linear regression leads to a validation of the model like,

$$
\widetilde{\Gamma}_{\mathrm{ICB}}^{1}(\omega)=K_{1} \times \Phi_{\mathrm{ic}}^{*} r_{\mathrm{i}}^{4} B_{r_{\mathrm{i}}}^{2}\left(\Omega_{\mathrm{oc}}-\Omega_{\mathrm{ic}}\right) / \mathcal{P}
$$

with

$$
\Phi_{\mathrm{ic}}^{*}\left(\delta_{\mathrm{c}}\right)=\left(\frac{1+i}{4}\right) \delta_{\mathrm{c}},
$$

the dimensionless transfer function, and

$$
K_{1}=1.14
$$

Here $\delta_{\mathrm{c}}$ is the dimensionless skin depth, the scaling law may than be written

$$
\widetilde{\Gamma}_{\mathrm{ICB}}^{1}(\omega)=K_{1} \times\left(\frac{1+i}{4}\right) \sqrt{\frac{2}{\omega}} r_{\mathrm{i}}^{4} B_{r_{\mathrm{i}}}^{2}\left(\Omega_{\mathrm{oc}}-\Omega_{\mathrm{ic}}\right) / \mathcal{P} .
$$




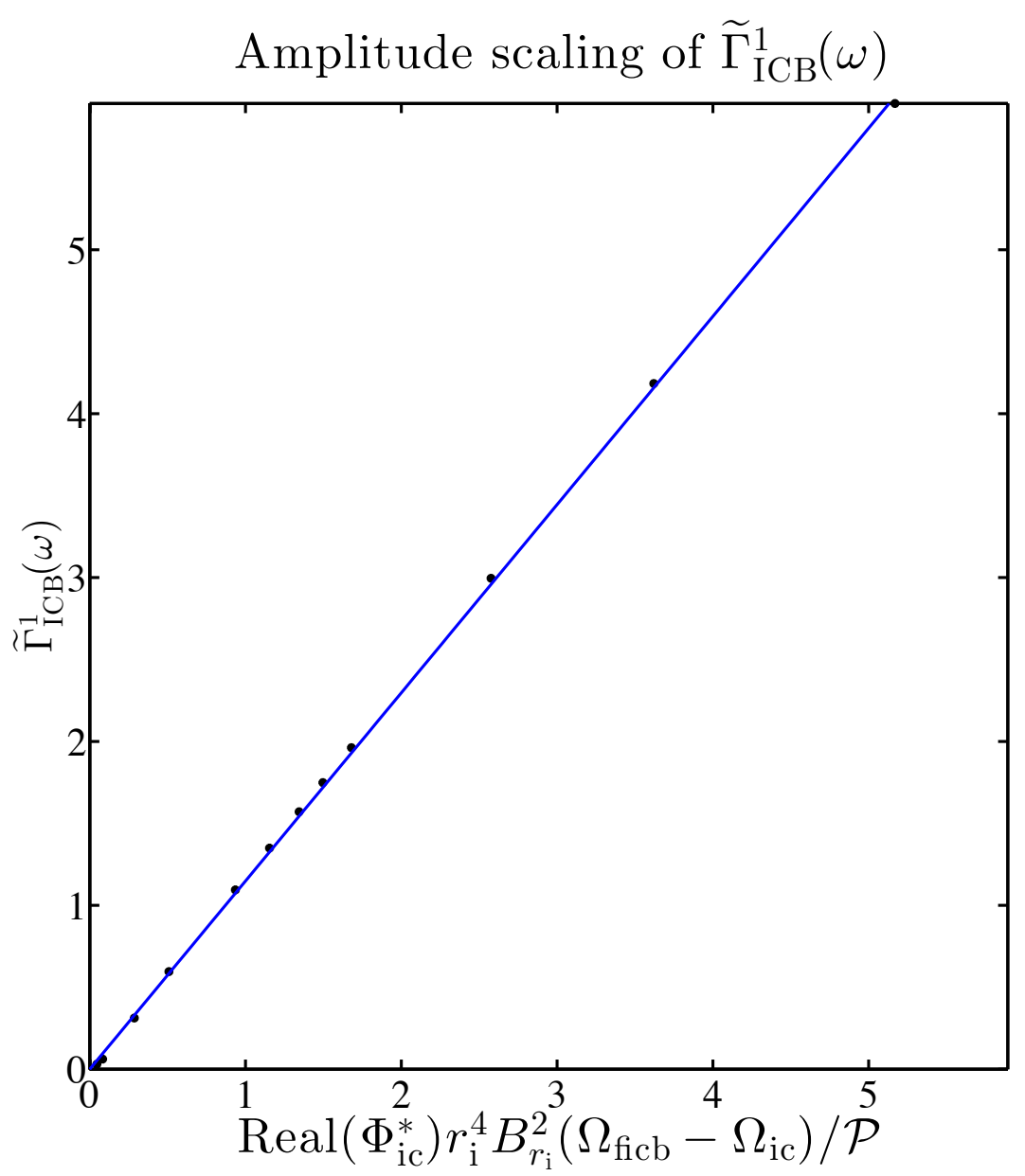

Figure 6.4: Scaling of the amplitude of the dimensionless electromagnetic torque exerted on the inner core $\widetilde{\Gamma}_{\text {ICB }}^{1}$, with a set of 12 numerical simulation with an imposed solid-body rotation of the outer with frequencies $f$ ranging from 1 to 1000 . The expression of $\Phi_{\mathrm{ic}}^{*}$ is given by equation (6.10). The blue line represents the best fit, with a slope of 1.14 .

\subsubsection{With gravitational coupling}

This section is dedicated to the analysis of the 20 simulations including a gravitational coupling between the inner core and the mantle, with an imposed periodic solid-body rotation in the outer core with frequency $f$ ranging from 1 to 10000 . The dimensionless gravitational torque $\Gamma_{\mathrm{G}}$ is implemented like

$$
\Gamma_{\mathrm{G}}=\zeta_{\eta}\left(\Omega_{\mathrm{ic}}-\Omega_{\mathrm{m}}\right)
$$

in which the strength of the gravitational coupling is embodied by the dimensionless number

$$
\zeta_{\eta}=\frac{\tau \Gamma}{\rho \eta_{\mathrm{c}} D^{3}}
$$


with $\tau$ a characteristic time scale of the viscous deformations of the inner core and $\Gamma$ a constant that accounts for the mantle heterogeneities of density. The 20 case-study simulations are separated into two series, 10 simulations with $\zeta_{\eta}=2 \times 10^{4}$ and 10 simulations with $\zeta_{\eta}=1 \times 10^{2}$. Figure 6.5 and 6.6 respectively present the magnitude of the electromagnetic torque and the shear at the ICB as a function of the angular frequency of the solid-body rotation of the outer core, in black for the simulations with $\zeta_{\eta}=2 \times 10^{4}$ and in red for the simulations with $\zeta_{\eta}=1 \times 10^{2}$. Figure 6.5 shows that, for a strong gravitational coupling

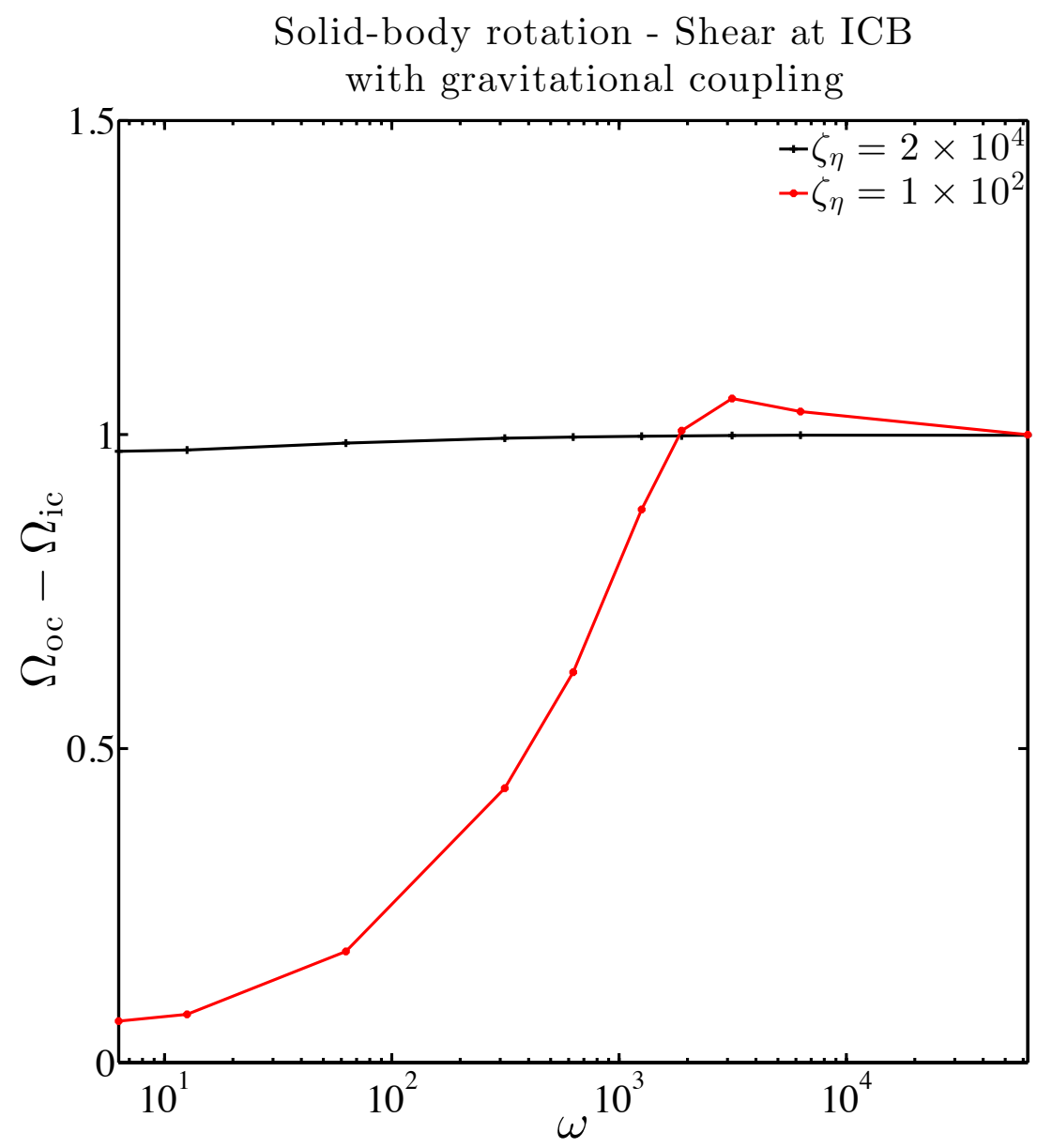

Figure 6.5: Amplitude of the shear at the ICB as a function of the angular frequency $\omega$ of the solid-body rotation imposed in the outer core. In black for the simulations with $\zeta_{\eta}=2 \times 10^{4}$ and in red for the simulations with $\zeta_{\eta}=1 \times 10^{2}$.

$\left(\zeta_{\eta}=2 \times 10^{4}\right)$, the inner core is always locked to the mantle, so that $\Omega_{\mathrm{oc}}-\Omega_{\mathrm{ic}} \approx \Omega_{\mathrm{oc}}$. However, for lower gravitational coupling constant $\left(\zeta_{\eta}=1 \times 10^{2}\right)$, the frequency dependence of the response of the inner core is still apparent. At low frequencies, i.e periods on the order of the magnetic diffusive time of the core, the inner core is entrained by the outer core so that the shear at the ICB is close to zero. Also, as the frequency increases, the inner core is less and less sensitive to the outer core rotation and the velocity jump at the ICB increases, a behavior at high frequencies that is similar to that noticed in absence of gravitational coupling (Fig. 6.3). Figure 6.6 expresses that the amplitude of the electromagnetic torque at the ICB decreases as the frequency of the outer core rotation increases to finally reach 


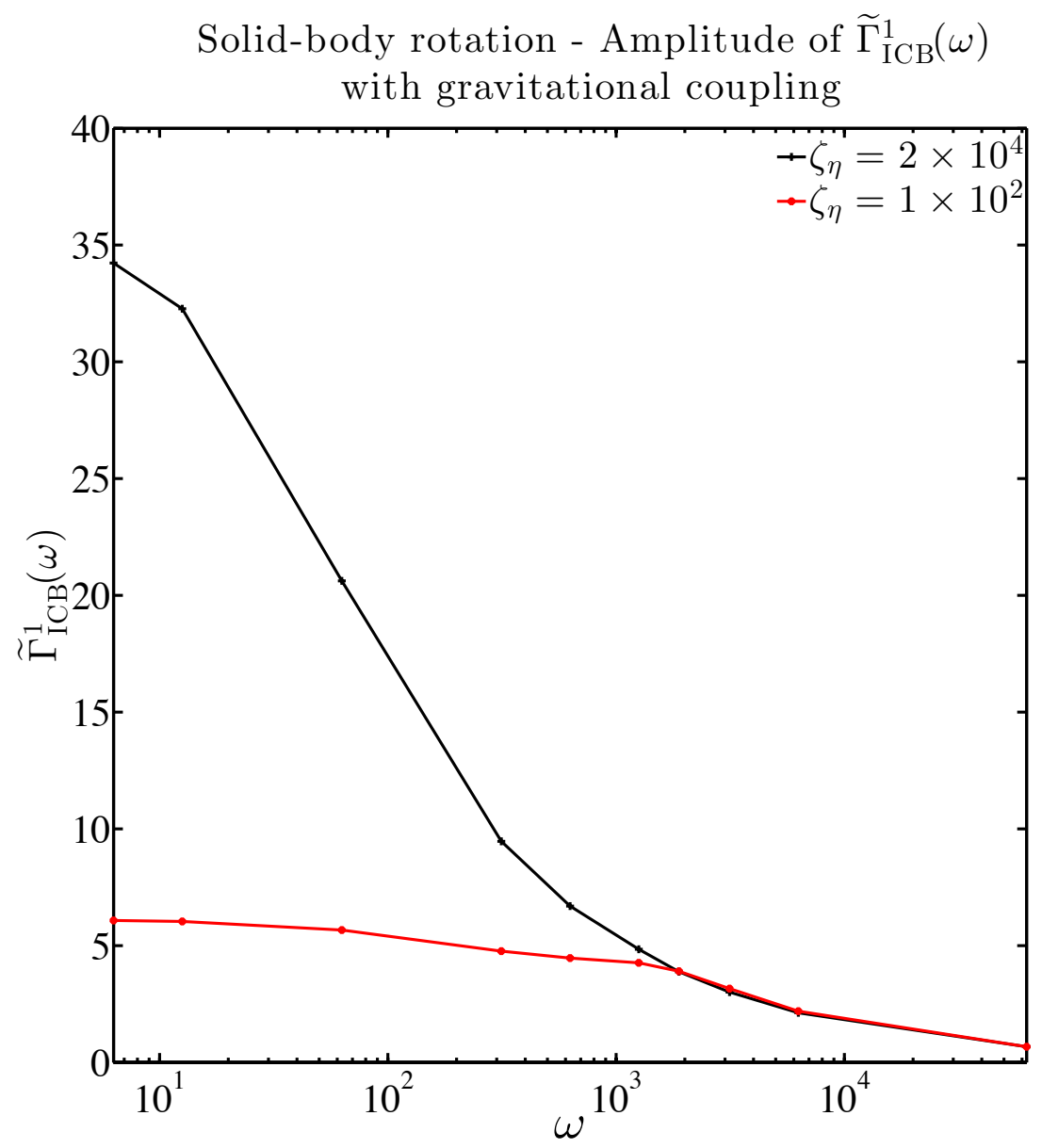

Figure 6.6: Amplitude of the electromagnetic torque at the ICB as a function of the angular frequency $\omega$ of the solid-body rotation imposed in the outer core. In black for the simulations with $\zeta_{\eta}=2 \times 10^{4}$ and in red for the simulations with $\zeta_{\eta}=1 \times 10^{2}$.

the same values as in the absence of gravitational coupling (Fig. 6.3). The influence of the gravitational coupling on the electromagnetic torque is best seen in figure 6.7, that pictures the scaling of the electromagnetic torque at the ICB with a gravitational coupling. At low frequencies, corresponding to high values of the electromagnetic torque, both sets of case-study simulations attain a threshold value illustrated by the horizontal black dashedlines. This reveals that, by limiting the inner core rotation, the gravitational torque also restricts the amplitude of the electromagnetic torque, the threshold value being larger as the coupling constant $\zeta_{\eta}$ is greater. Fortunately, this limit is only observed at very-low frequencies, corresponding to periods higher than 30000 years. The blue line in figure 6.7 represents the best-fit for $\widetilde{\Gamma}_{\mathrm{ICB}}^{1}$ when removing the very-low frequency simulations. As in the absence of gravitational coupling, the scaling obeys

$$
\widetilde{\Gamma}_{\mathrm{ICB}}^{1}(\omega)=K_{1} \times\left(\frac{1+i}{4}\right) \sqrt{\frac{2}{\omega}} r_{\mathrm{i}}^{4} B_{r_{\mathrm{i}}}^{2}\left(\Omega_{\mathrm{oc}}-\Omega_{\mathrm{ic}}\right) / \mathcal{P},
$$

with $K_{1}=1.14$, suggesting that, for periods lower than 30000 years, the model of the electromagnetic torque acting on the inner is appropriate to describes the system. 


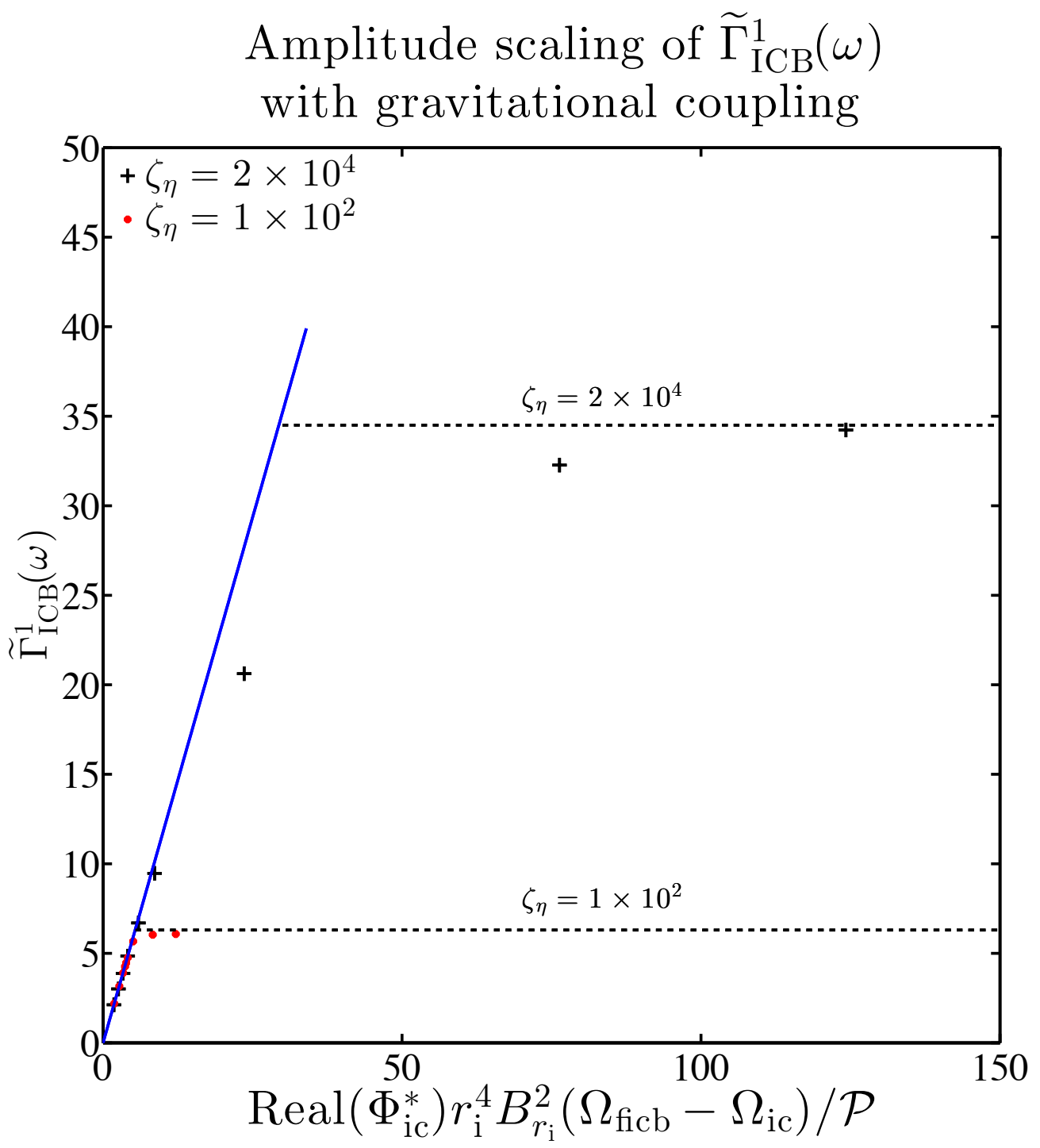

Figure 6.7: Scaling of the dimensionless perturbed electromagnetic torque at the ICB $\widetilde{\Gamma}_{\text {ICB }}^{1}$, with two sets of 10 numerical case-study simulations respectively with a gravitational coupling constant $\zeta_{\eta}=2 \times 10^{4}$, in black, and $\zeta_{\eta}=1 \times 10^{2}$, in red. The outer core is solid-body rotating at frequencies ranging from 1 to 10000 and the expression of $\Phi_{\mathrm{ic}}^{*}$ is given by equation (6.10). The blue line represents the best fit, without the very-low frequency simulations $(f<10)$, with a slope of 1.14 .

\subsubsection{Expressions of $\mathcal{S}$ and $\mathcal{D}$}

We here want to establish the expressions of the inner core super-rotation $\mathcal{S}$ and the geomagnetic westward drift $\mathcal{D}$ in the case of a solid-body rotation of the outer core. To do so, we first re-write the conservation of angular momentum (6.1) in terms of Fourier transforms. The conservation of the angular momentum of the inner core becomes

$$
\begin{aligned}
\mathrm{I}_{\mathrm{i}} \frac{1}{\sqrt{2 \pi}} \frac{\mathrm{d}}{\mathrm{dt}} \int_{-\infty}^{+\infty} \Omega_{\mathrm{ic}}(\omega) e^{-i \omega t} \mathrm{~d} \omega & =\frac{1}{\sqrt{2 \pi}} \int_{-\infty}^{+\infty} \widetilde{\Gamma}_{\mathrm{ICB}}^{1}(\omega) e^{-i \omega t} \mathrm{~d} \omega \\
\tau & \Gamma \frac{1}{\sqrt{2 \pi}} \int_{-\infty}^{+\infty}\left(\Omega_{\mathrm{ic}}(\omega)-\Omega_{\mathrm{m}}(\omega)\right) e^{-i \omega t} \mathrm{~d} \omega
\end{aligned}
$$


when using the same notation of the angular velocity of rotation in the frequency and time domains. For each angular frequency, this can be reduced to the dimensionless expression,

$$
-\mathrm{I}_{\mathrm{i}} i \omega \Omega_{\mathrm{ic}}=\mathcal{L} u^{2} K_{1}\left(\frac{1+i}{4}\right) \sqrt{\frac{2}{\omega}} r_{\mathrm{i}}^{4} B_{r_{\mathrm{i}}}^{2}\left(\Omega_{\mathrm{oc}}-\Omega_{\mathrm{ic}}\right)-\zeta_{\eta}\left(\Omega_{\mathrm{ic}}-\Omega_{\mathrm{m}}\right),
$$

by adopting the previously validated model for the perturbed electromagnetic torque at the ICB (Eq. 6.12). An analogous equation can be formulated for the dimensionned conservation of the angular momentum of the solid mantle,

$$
\begin{aligned}
\mathrm{I}_{\mathrm{m}} \frac{1}{\sqrt{2 \pi}} \frac{\mathrm{d}}{\mathrm{dt}} \int_{-\infty}^{+\infty} \Omega_{\mathrm{m}}(\omega) e^{-i \omega t} \mathrm{~d} \omega & =\frac{1}{\sqrt{2 \pi}} \int_{-\infty}^{+\infty} \widetilde{\Gamma}_{\mathrm{CMB}}^{1}(\omega) e^{-i \omega t} \mathrm{~d} \omega \\
& +\tau \Gamma \frac{1}{\sqrt{2 \pi}} \int_{-\infty}^{+\infty}\left(\Omega_{\mathrm{ic}}(\omega)-\Omega_{\mathrm{m}}(\omega)\right) e^{-i \omega t} \mathrm{~d} \omega
\end{aligned}
$$

that again, for each angular frequency, can be reduced to following dimensionless expression

$$
-\mathrm{I}_{\mathrm{m}} i \omega \Omega_{\mathrm{m}}=\mathcal{L} u^{2} K_{2} r_{\mathrm{o}}^{4} B_{r_{\mathrm{o}}}^{2} \Sigma\left(\Omega_{\mathrm{oc}}-\Omega_{\mathrm{m}}\right)+\zeta_{\eta}\left(\Omega_{\mathrm{ic}}-\Omega_{\mathrm{m}}\right)
$$

using the dimensionless model for the perturbed electromagnetic torque at the CMB (see chapter 5) and introducing the dimensionless conductance of the electrically conducting layer at the base of the mantle

$$
\Sigma=\frac{\sigma_{\mathrm{m}} \Delta}{\sigma_{\mathrm{c}} D}
$$

As anticipated, our choice of the characteristic dimensions of the system introduces the Lundquist number as control parameter for the angular conservation of the inner core and the mantle, in equations (6.20) and (6.17). In addition, this set of equations now allows the formulations of the angular velocities of the inner core and the mantle, $\Omega_{\text {ic }}$ and $\Omega_{\mathrm{m}}$, as functions of the imposed solid-body rotation of the outer core. Do to so, we first simplify the notation of the set that becomes

$$
\begin{aligned}
& -i \omega \Omega_{\mathrm{ic}}=\Psi_{1}\left(\Omega_{\mathrm{oc}}-\Omega_{\mathrm{ic}}\right)-\Psi_{\mathrm{ic}}\left(\Omega_{\mathrm{ic}}-\Omega_{\mathrm{m}}\right), \\
& -i \omega \Omega_{\mathrm{m}}=\Psi_{2}\left(\Omega_{\mathrm{oc}}-\Omega_{\mathrm{m}}\right)+\Psi_{\mathrm{m}}\left(\Omega_{\mathrm{ic}}-\Omega_{\mathrm{m}}\right),
\end{aligned}
$$

with

$$
\begin{array}{ll}
\Psi_{1}=\frac{K_{1}\left(\frac{1+i}{4}\right) \sqrt{\frac{2}{\omega}} r_{\mathrm{i}}^{4} B_{r_{\mathrm{i}}}^{2} \mathcal{L} u^{2}}{\mathrm{I}_{\mathrm{i}}}, & \Psi_{\mathrm{ic}}=\frac{\zeta_{\eta}}{\mathrm{I}_{\mathrm{i}}} \\
\Psi_{2}=\frac{K_{2} r_{\mathrm{o}}^{4} B_{r_{\mathrm{o}}}^{2} \Sigma \mathcal{L} u^{2}}{\mathrm{I}_{\mathrm{m}}}, & \Psi_{\mathrm{m}}=\frac{\zeta_{\eta}}{\mathrm{I}_{\mathrm{m}}}
\end{array}
$$

After a few demonstration steps (see A.3), the angular velocity of the mantle can be described like

$$
\Omega_{\mathrm{m}}=\Omega_{\mathrm{oc}}\left(\frac{\Psi_{2} \gamma_{c}+\Psi_{\mathrm{m}} \Psi_{1}}{\gamma_{c} \gamma_{m}-\Psi_{\mathrm{ic}} \Psi_{\mathrm{m}}}\right)
$$


with

$$
\gamma_{c}=\Psi_{1}+\Psi_{\mathrm{ic}}-i \omega, \quad \text { and } \quad \gamma_{m}=\Psi_{2}+\Psi_{\mathrm{m}}-i \omega
$$

or in terms of the geomagnetic westward drift $\mathcal{D}=\Omega_{\mathrm{oc}}-\Omega_{\mathrm{m}}$,

$$
\mathcal{D}=\Omega_{\mathrm{oc}}\left(1-\frac{\Psi_{2} \gamma_{c}+\Psi_{\mathrm{m}} \Psi_{1}}{\gamma_{c} \gamma_{m}-\Psi_{\mathrm{ic}} \Psi_{\mathrm{m}}}\right)
$$

In addition, the angular velocity of rotation of the inner core follows

$$
\Omega_{\mathrm{ic}}=\Omega_{\mathrm{oc}}\left(\frac{\Psi_{1} \gamma_{m}+\Psi_{\mathrm{ic}} \Psi_{2}}{\gamma_{c} \gamma_{m}-\Psi_{\mathrm{ic}} \Psi_{\mathrm{m}}}\right)
$$

leading to a super-rotation of the inner core $\mathcal{S}=\Omega_{\mathrm{ic}}-\Omega_{\mathrm{m}}$ of the form

$$
\mathcal{S}=\Omega_{\mathrm{oc}}\left(\frac{\Psi_{1} \gamma_{m}+\Psi_{\mathrm{ic}} \Psi_{2}-\Psi_{2} \gamma_{c}-\Psi_{\mathrm{m}} \Psi_{1}}{\gamma_{c} \gamma_{m}-\Psi_{\mathrm{ic}} \Psi_{\mathrm{m}}}\right)
$$

Figure 6.8 exposes the comparison between the results of the numerical case-study simulations, in blue, and equation (6.28), in black, in terms of the amplitude of the inner core super rotation $\mathcal{S}$ (top) and the phase lag (bottom) between $\mathcal{S}$ and the solid-body rotation of the outer core $\Omega_{\mathrm{oc}}$. The agreement between the numerical simulations and the analytical expression is satisfying, especially at both low and high frequencies. At low frequency, i.e. when the period of the forcing is much greater than the Alfvén time, the inner core follows the fluid outer core: the inner core super-rotation equals the amplitude of the perturbation with no lag between the two. At high frequencies, the period of the forcing becomes small compared to the Alfvén time such that the inner core becomes less and less sensitive to the azimuthal velocity perturbation, leading to a vanishing inner core super-rotation. Between these two regimes, i.e when the period is of the order of the Alfvén time, we can observe the transition with an offset between the model and the simulations. In the dimensionless formalism, the period of the forcing is on the order of the Alfvén time corresponds to $\omega \simeq 2 \pi \mathcal{L} u$. Given our value of the Lundquist number, we obtain a transition around $\omega \simeq 270$, which actually coincides with the very begin of the transition in figure 6.8. Pursuant to this reasoning, is it possible to anticipate this transition for Earth, or at least of the solid-body rotation part of the earth core. Considering that the Lundquist number of the Earth's core is on the order of $10^{4}$, the transition should then occur around forcing periods of 30 years.

In the presence of the gravitational coupling between the inner core and the mantle, as pictured by figure 6.9, this offset is still present. However, the mismatch between the numerical results (dashed lines) and the analytical solutions (plain lines) is reduced by the increase of the gravitational coupling constant $\zeta_{\eta}$, that characterizes the strength of the gravitational coupling.

In addition, figure 6.9 shows that the presence of gravitational coupling does not 


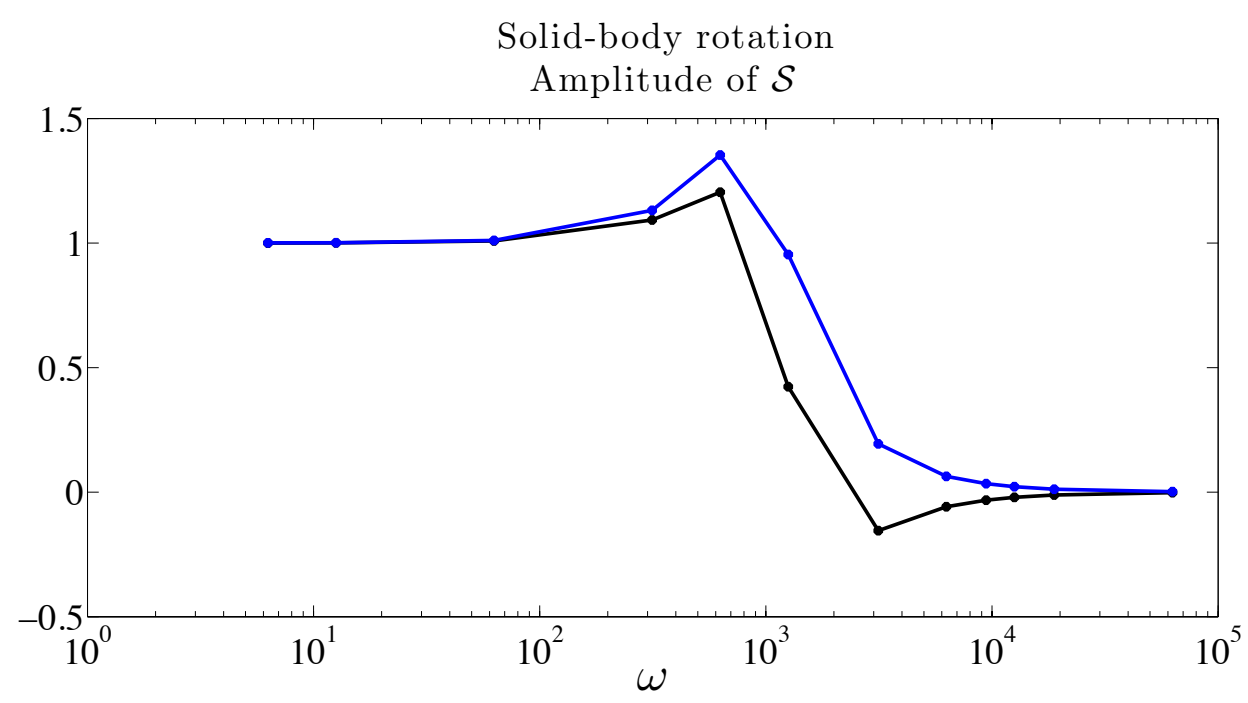

Phase lag between $\mathcal{S}$ and $\Omega_{\mathrm{oc}}$

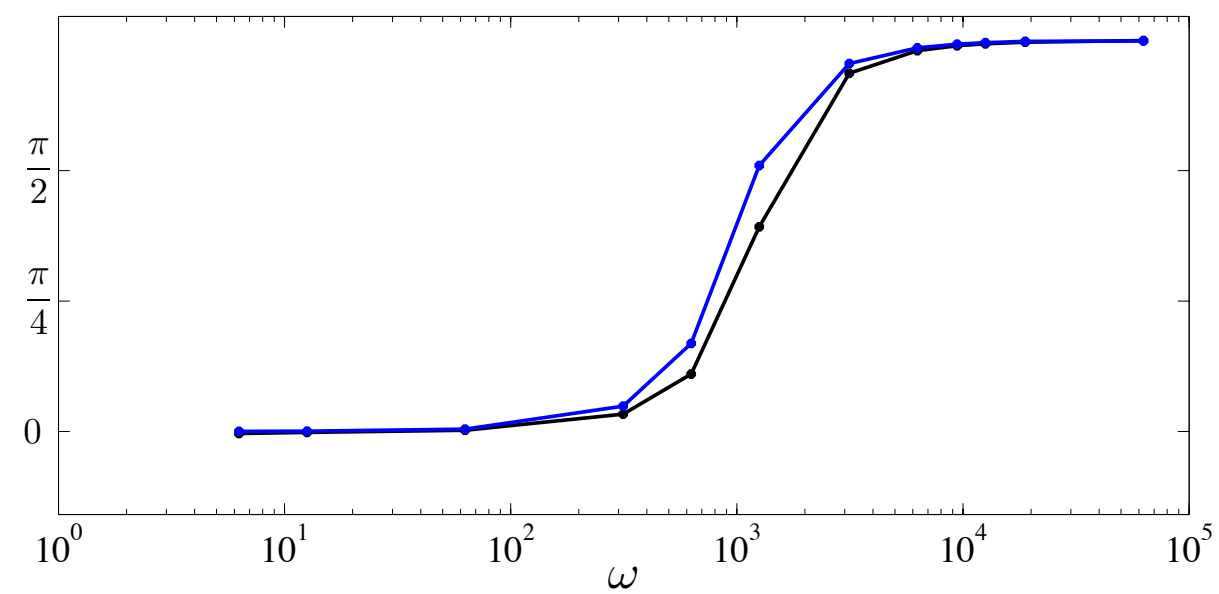

Figure 6.8: Amplitude (top) and phase-lag (bottom) of the inner core super rotation $\mathcal{S}=\Omega_{\text {ic }}-\Omega_{\mathrm{m}}$ as a function of the angular frequency $\omega$ in absence of gravitational coupling. In blue, the observation in the numerical simulations and in black the solution given by equation (6.28).

drastically affect the previously identified behavior of inner core super-rotation as a function of the forcing frequency. Indeed, for $\zeta_{\eta}=1 \times 10^{2}$ in red, we can still notice the two different regimes, at the exception of a slightly smaller co-rotation at low frequency. This argument is of course no longer valid for stronger gravitational coupling constant, in black, that completely prevent any inner core differential rotation.

\subsection{Shear in the fluid outer core}

\subsubsection{Shear flow}

The previous section focuses on the scaling of the electromagnetic torque at the inner core boundary initiated by the velocity jump between the angular rotation of the inner core and the imposed solid-body rotation of the outer core. However, though this establishes the first approach toward the scaling of the electromagnetic torque as a function of the velocity 
Solid-body rotation - with gravitational coupling

Amplitude of $\mathcal{S}$

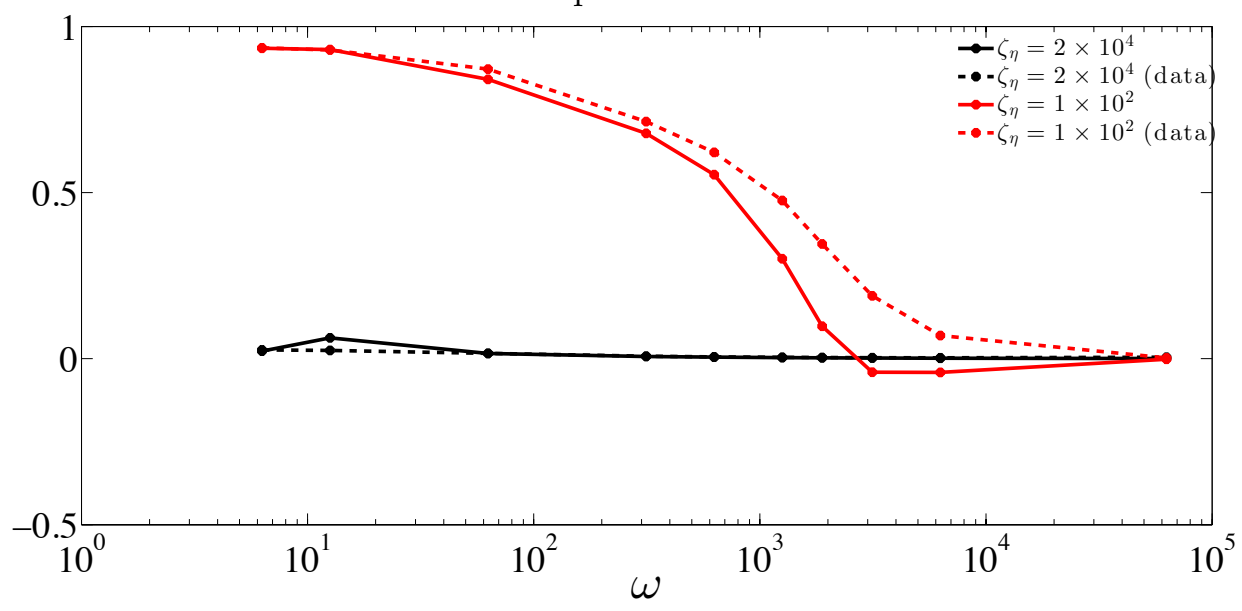

Phase lag between $\mathcal{S}$ and $\Omega_{\text {oc }}$

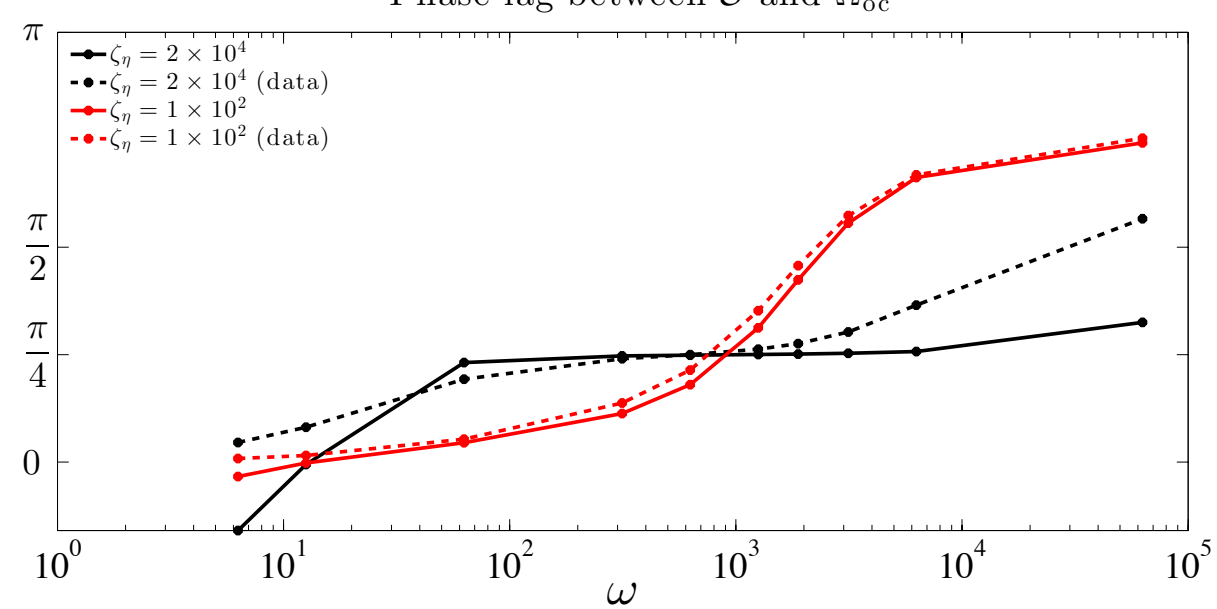

Figure 6.9: Amplitude (top) and phase-lag (bottom) of the inner core super rotation $\mathcal{S}=\Omega_{\text {ic }}-\Omega_{\mathrm{m}}$ as a function of the angular frequency $\omega$ with a gravitational coupling, in black for $\zeta_{\eta}=2 \times 10^{4}$ and in red for $\zeta_{\eta}=1 \times 10^{2}$. The dashed lines are the observations in the numerical simulations while the plain lines represent the solutions given by equation (6.28).

jump, it overlooks the influence of the shear in the fluid outer core. Indeed, Pichon et al. (2016) proved that the expression of the long-term electromagnetic torque features a local (velocity jump at the ICB) and a remote contribution from the shear in the fluid outer core, namely $\mathcal{C}_{\mathrm{f}}=\Omega_{\mathrm{ficb}}-\Omega_{\mathrm{fcmb}}$. Yet, imposing a solid-body rotation in the outer core is identical to setting $\mathcal{C}_{\mathrm{f}}=0$.

In order to investigate the influence of $\mathcal{C}_{\mathrm{f}}$ on the electromagnetic torque acting on the inner core, we performed a series of 20 numerical simulations in which we imposed a periodic flow in the fluid core that bears no angular momentum. In other words, we chose to impose a flow into the fluid outer core of the form

$$
\omega_{\mathrm{f}}(r)=a+b\left(r-r_{\mathrm{o}}\right),
$$


that has to force the angular momentum of the fluid outer core to be zero,

$$
\int_{V_{f}} \omega_{\mathrm{f}} \mathrm{i}_{\mathrm{f}} \mathrm{d} V=0
$$

We found that, in order to satisfy this constraint, the flow in the fluid outer should correspond to

$$
\omega_{\mathrm{f}}(r)=a\left[1+\left(r-r_{\mathrm{o}}\right) \frac{6\left(r_{\mathrm{o}}^{5}-r_{\mathrm{i}}^{5}\right)}{r_{\mathrm{o}}^{6}-r_{\mathrm{i}}^{5}\left(5 r_{\mathrm{i}}-6 r_{\mathrm{o}}\right)}\right],
$$

with $a$ the value of the angular velocity of the fluid at the core-mantle boundary $\left(r=r_{\mathrm{o}}\right)$. Figure 6.10 shows the form of the imposed shear flow in the fluid outer, along with the poloidal magnetic field lines and the azimuthal magnetic field produced, on the right. The snapshots are extracted from a case-study simulation with a frequency $f=10$ of the periodic forcing in the outer core, picturing a strong azimuthal magnetic field inside the fluid outer core.
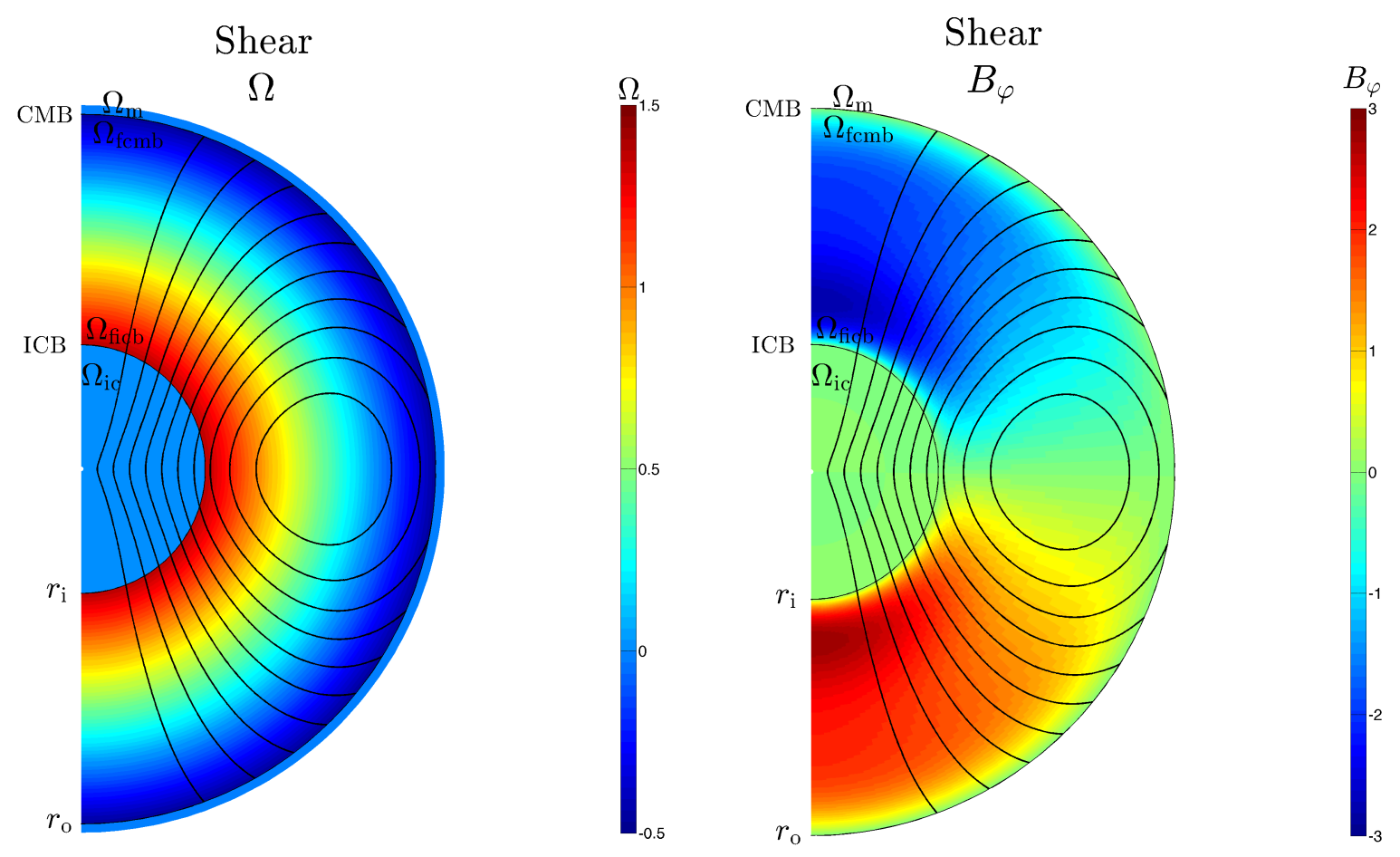

Figure 6.10: Snapshots of a case-study simulation with an imposed shear flow in the outer core and a static poloidal field (black lines). On the left, the angular velocities of the inner core $\Omega_{\mathrm{ic}}$, the mantle $\Omega_{\mathrm{m}}$, and the fluid close to the ICB and CMB, $\Omega_{\mathrm{ficb}}$ and $\Omega_{\mathrm{fcmb}}$. On the right, the azimuthal magnetic field produced by the velocity jumps at ICB and CMB.

Figure 6.11 presents the amplitudes of the shear, $\Omega_{\text {ficb }}-\Omega_{\text {ic }}$, and the electromagnetic torque at the ICB, $\widetilde{\Gamma}_{\text {ICB }}^{1}$. As in the solid-body rotation experiments (see Fig. 6.3), the amplitude of the perturbed electromagnetic torque at the ICB is low at the very low frequencies. However, at those same very low frequencies the shear at the ICB is no longer zero. In this frequency domain, we thus observe a strong shear at the ICB that does not lead to a strong electromagnetic torque acting on the inner core, and consequently a vanishing 
inner core super rotation. This demonstrates that, for low frequencies, the inner core is non longer sensitive to the azimuthal magnetic field produced by the velocity jumps at the ICB. In other words, the azimuthal magnetic field produced by the remote shear in the body of the outer core overcomes the local velocity jump effect.

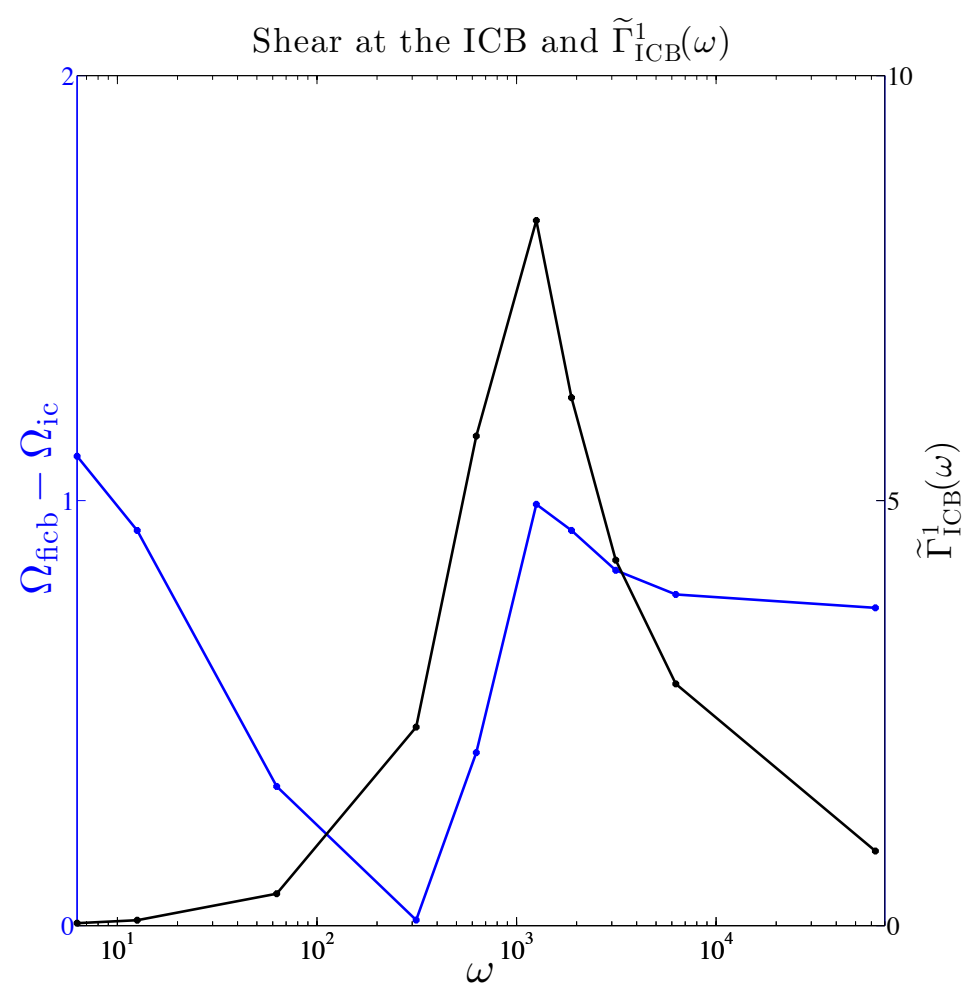

Figure 6.11: Shear at the ICB, $\Omega_{\text {ficb }}-\Omega_{\text {ic }}$ and amplitude of the electromagnetic torque acting on the inner core $\widetilde{\Gamma}_{\mathrm{ICB}}^{1}$, as a function of the angular frequency $\omega$ of the periodic imposed shear flow in the fluid outer core.

This change on the behavior can also be observed in figure 6.12, that exposes the phase lag between the shear and the electromagnetic torque at the inner core boundary. At low frequencies the phase lag is strong, leading to a strong lag between the torque and the inner core angular rotation. However, as we increase the frequency of the periodic shear flow in the fluid outer core, we recover a phase lag that tends to reach the $\frac{\pi}{4}$ value.

Finally, the influence of a shear in the fluid outer core is best seen in figure 6.13, that compares the azimuthal field only produced by the velocity jumps in the solid-body rotation case-study simulations (top) and the azimuthal field produced by a combination of the velocity jumps and a shear in the fluid outer core (bottom). In the solid body-rotation simulations, we observed that as the angular frequency of rotation of the outer core increases, the inner core lag increases as well. This induces larger velocity jumps at the fluid core boundaries and consequently a vigorous production of azimuthal magnetic field, notably at the inner core boundary, that is visible on the snapshots on the top of figure 6.13 . The snapshots on the bottom of figure 6.13 are extracted from the shear flow case-study simulations. At low frequency, they exhibit a strong azimuthal magnetic field in the body of the fluid outer core, that do no reach the boundaries. However, as we increase the frequency 


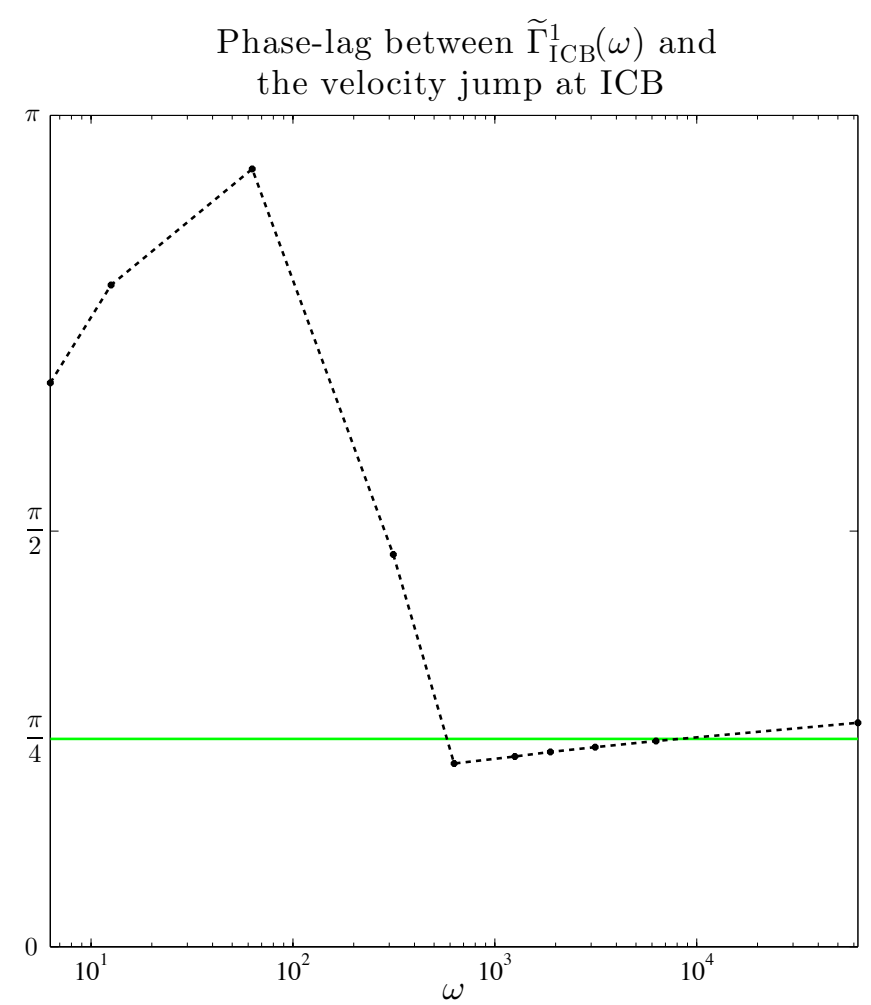

Figure 6.12: Phase lag at the ICB as a function of the angular frequency $\omega$ of the periodic imposed shear flow in the fluid outer core. In green, the previously identified limit value of $\frac{\pi}{4}$.

of oscillations in the fluid outer core, the amplitude of the azimuthal field produced decreases. More importantly, the azimuthal field produced is more and more located at the fluid core boundaries.

These observations lead to a scaling of the electromagnetic torque that sets aside the very low frequency case-study simulations, namely with frequencies $f<100$. For frequencies higher than 100, corresponding to periods lower than 3000 years, the electromagnetic torque can be scaled as in section 6.2. This scaling is displayed by figure 6.14 and again validates the model of the electromagnetic torque acting on the inner core defined by equation (6.12). This scaling also displays the agreement with 10 simulations that include gravitational coupling $\left(\zeta_{\eta}=2 \times 10^{4}\right)$, picturing the same plateau value identified in the solid-body rotation case-study simulations in section 6.2.3.

\subsubsection{Expressions of $\mathcal{S}$ and $\mathcal{D}$}

We now wish to establish the expressions of inner core super rotation and the geomagnetic westward drift as functions of the shear imposed in the fluid outer core. Following the same steps as in section 6.2.4, and including the angular velocities of the fluid close to the ICB and close to the CMB, respectively $\Omega_{\text {ficb }}$ and $\Omega_{\mathrm{fcmb}}$, the conservation of the angular 
Azimuthal magnetic field $B_{\varphi}$
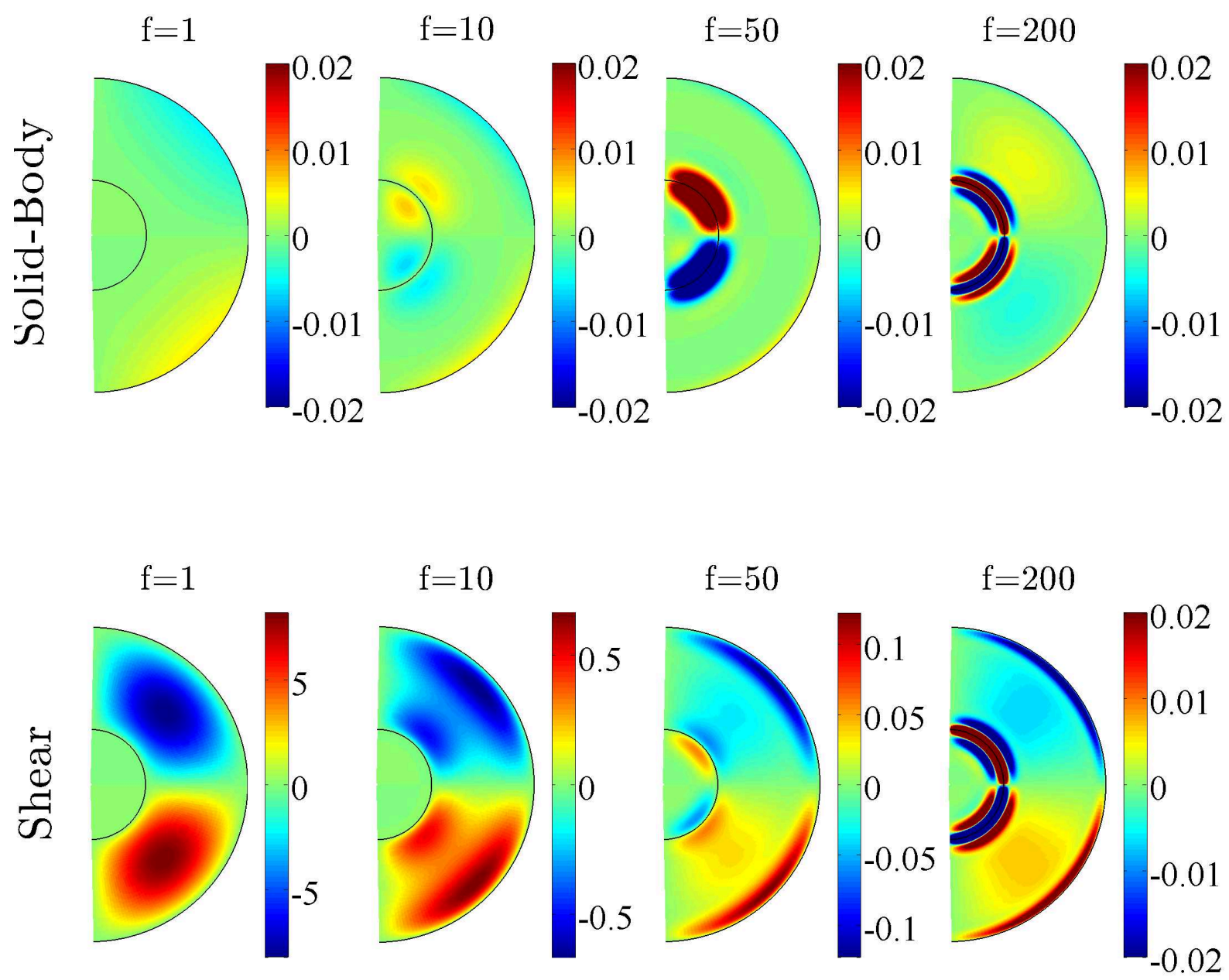

Figure 6.13: Snapshots of the azimuthal magnetic field $B_{\varphi}$ in the fluid outer and in the inner core, in the case study simulations with a forced outer core periodic solid-body rotation (on top) and a forced shear flow in the fluid outer core (bottom), without gravitational coupling and for frequencies of periodic forcing $f=1, f=10, f=50$ and $f=200$.

momentum of the system becomes

$$
\begin{aligned}
& -i \omega \Omega_{\mathrm{ic}}=\Psi_{1}\left(\Omega_{\mathrm{ficb}}-\Omega_{\mathrm{ic}}\right)-\Psi_{\mathrm{ic}}\left(\Omega_{\mathrm{ic}}-\Omega_{\mathrm{m}}\right), \\
& -i \omega \Omega_{\mathrm{m}}=\Psi_{2}\left(\Omega_{\mathrm{fcmb}}-\Omega_{\mathrm{m}}\right)+\Psi_{\mathrm{m}}\left(\Omega_{\mathrm{ic}}-\Omega_{\mathrm{m}}\right) \text {, } \\
& 0=-\Psi_{1} \mathrm{I}_{\mathrm{i}}\left(\Omega_{\mathrm{ficb}}-\Omega_{\mathrm{ic}}\right)-\Psi_{2} \mathrm{I}_{\mathrm{m}}\left(\Omega_{\mathrm{fcmb}}-\Omega_{\mathrm{m}}\right) .
\end{aligned}
$$

Solving this system (see A.3) leads to the formulation of the time-dependent expressions of the inner core super rotation

$$
\mathcal{S}=\mathcal{C}_{\mathrm{f}} \frac{\Psi_{1}-\gamma_{\mathrm{f}}\left(\Psi_{1}-\Psi_{2}\right)}{\Psi_{1}+\Psi_{\mathrm{ic}}+\Psi_{\mathrm{m}}-\gamma_{\mathrm{f}}\left(\Psi_{1}-\Psi_{2}\right)-i \omega}
$$




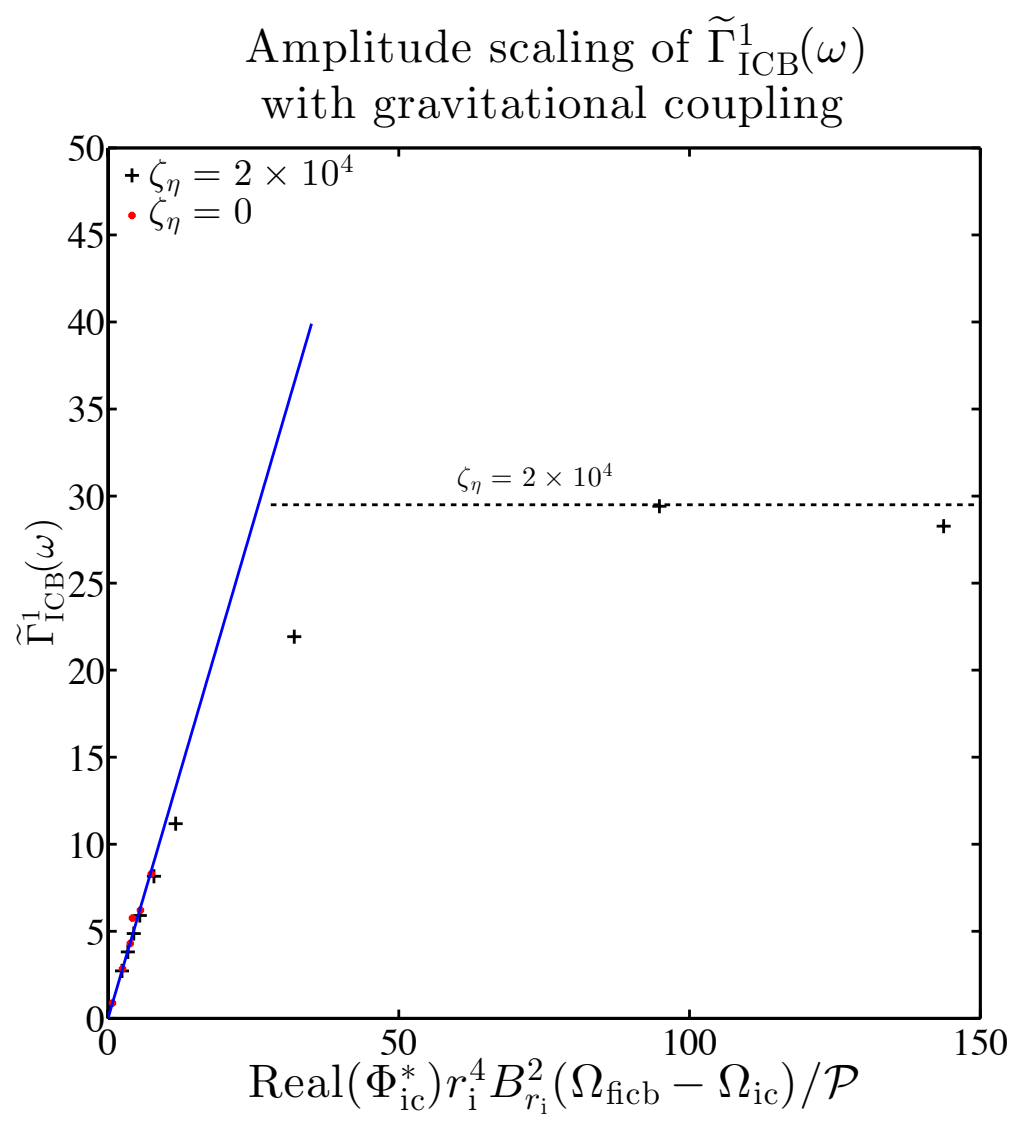

Figure 6.14: Scaling of the electromagnetic torque acting on the inner core $\widetilde{\Gamma}_{\text {ICB }}^{1}$ in numerical case-study simulations with a periodic shear flow imposed into the fluid outer core. $\Phi_{\mathrm{ic}}^{*}$ is given by equation (6.10). The blue line represents the best fit, without the very-low frequency simulations $(f<100)$, with a slope of 1.14 . Red points represents the 9 simulations without gravitational coupling while the black cross are the 9 simulations with $\zeta_{\eta}=2 \times 10^{4}$.

and the geomagnetic westward drift

$$
\mathcal{D}=-\mathcal{C}_{\mathrm{f}} \gamma_{\mathrm{f}}\left(1-\frac{\Psi_{1}-\gamma_{\mathrm{f}}\left(\Psi_{1}-\Psi_{2}\right)}{\Psi_{1}+\Psi_{\mathrm{ic}}+\Psi_{\mathrm{m}}-\gamma_{\mathrm{f}}\left(\Psi_{1}-\Psi_{2}\right)-i \omega}\right),
$$

as functions of the electromagnetic torques amplitudes embodied by $\Psi_{1}$ and $\Psi_{2}$, the moments of inertia of the inner core $I_{i}$ and the mantle $I_{m}$ and the angular frequency of the period forcing $\omega$ imposed in the fluid outer core, with

$$
\gamma_{\mathrm{f}}=\frac{\Psi_{1} \mathrm{I}_{\mathrm{i}}}{\Psi_{1} \mathrm{I}_{\mathrm{i}}+\Psi_{2} \mathrm{I}_{\mathrm{m}}}
$$

The agreement between the numerical results and the analytical form given by equation (6.33) is displayed in figure 6.15. This figure adds the results of 10 numerical simulations with a strong gravitational coupling between the inner core and the mantle implemented with $\zeta_{\eta}=2 \times 10^{4}$, in black. With and without a gravitational coupling, the effect of the shear inside the fluid outer core is apparent at low frequencies, leading to a significant mismatch between the numerical results and the outcome of equation (6.33) on the left side 
of the diagrams. Notwithstanding the already identified mismatch around the transition between the two regimes, the agreement is sufficient as the angular frequency of oscillation increases in the fluid outer core, particularly regarding the phase lag between the inner core super rotation and the shear in the fluid outer core, at the bottom of figure 6.15.

Shear flow $\mathcal{C}_{\mathrm{f}}$

Amplitude of $\mathcal{S}$

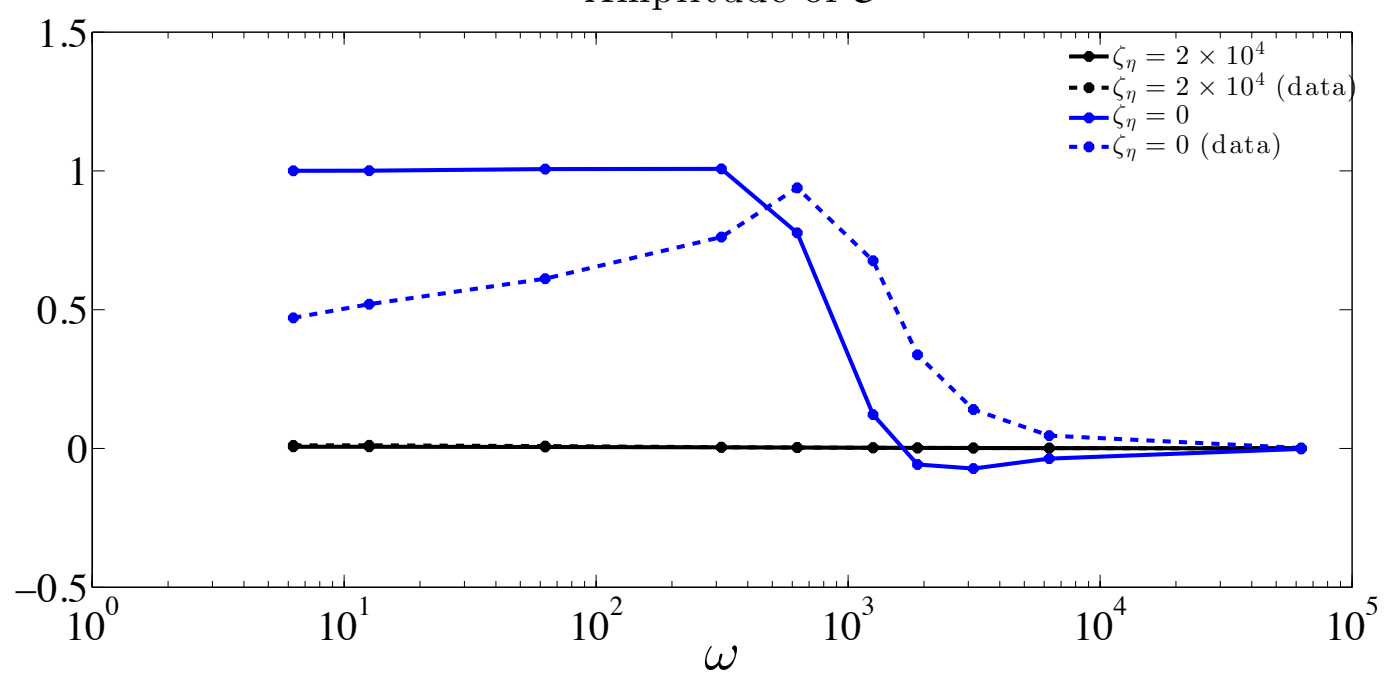

Phase lag between $\mathcal{S}$ and $\mathcal{C}_{\mathrm{f}}$

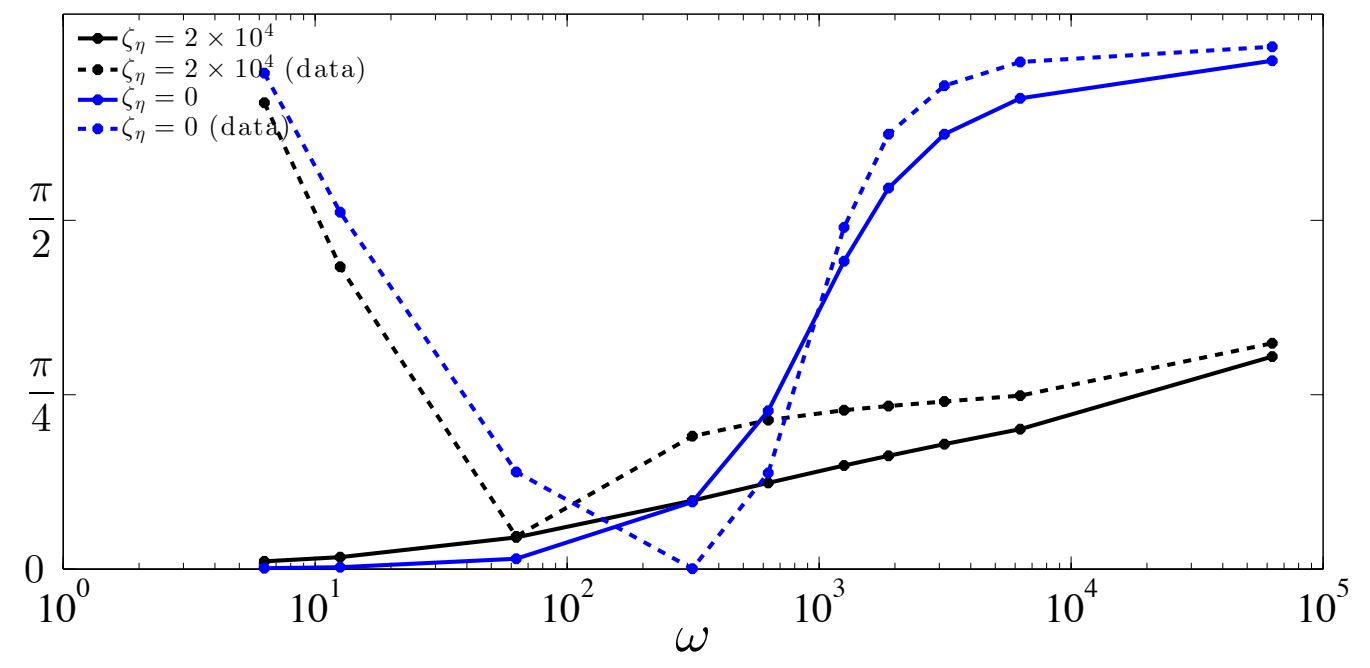

Figure 6.15: Amplitude (top) and phase-lag (bottom) of the inner core super rotation $\mathcal{S}=\Omega_{\mathrm{ic}}-\Omega_{\mathrm{m}}$ as a function of the angular frequency $\omega$ without gravitational coupling, in blue, and with gravitational coupling, in black with $\zeta_{\eta}=2 \times 10^{4}$. The dashed lines are the observations in the numerical simulations while the plain lines represent the solutions given by equation (6.33).

\subsection{Conclusion}

The analysis of the case-study simulation put lights on important aspects of the timedependent electromagnetic torque acting on the inner core. First, the systematic study on the solid-body rotation cases allowed the validations of the electromagnetic torque model 
at the ICB. We thus proved that our numerical set up is conformed with a time-dependent torque that is produced by the shear of a dipolar field by the velocity jump at the ICB. Second, we characterized the influence of a shear in the body of the fluid outer core, in simulations picturing a shear flow that bears no angular momentum. As the impact of the remote shear is only significant at periods close the characteristic magnetic diffusion time of the core $\tau_{\eta}$, we conclude that considering this global shear in a time-dependent approach is irrelevant. Third, we analyzed the consequences of the presence of gravitational coupling between the inner core and the mantle which induces a consequent deviation on the scaling at low frequencies. Finally, we were able to attain the consistency check addressed at the beginning of this section: to predict the time-dependent inner core super rotation in case-study simulations with a main, static, dipolar magnetic field and two different azimuthal oscillating flows imposed to the fluid outer core. 


\title{
Amplitude of rotational fluctuations in geodynamo simulations
}

\begin{abstract}
This chapter presents an analysis of time-dependent inner core - outer core - mantle system in numerical simulations of the geodynamo. We first compare the results of the simulations with the two ideal cases developed in the previous chapter. For this, we use the standard deviations of the four shears that characterize our system, as measures of the decadal to secular fluctuations of the differential rotation of the inner core. These oscillations seem mainly generated by a shear flow of the fluid core which does not carry, or very little, angular momentum. In addition, we observe that the parameter that most influences the rotation oscillations of the inner core is the intensity of the gravitational torque between the inner core and the mantle. These observations make it possible to constrain the amplitude of the decadal fluctuations of the differential rotation of the inner core as being less than $0.04^{\circ} \mathrm{yr}^{-1}$, a value nearly two orders of magnitude lower than that estimated by the seismological study conducted by Tkalčić et al. (2013).
\end{abstract}

\section{Résumé}

Ce chapitre est une première étape dans l'analyse des simulations numériques de la géodynamo en terme de dépendance temporelle du système couplé graine - noyau externe manteau. Nous comparons ici les résultats des simulations aux deux cas idéaux développés dans le chapitre précédent. Pour cela, nous utilisons les écarts-types des quatre cisaillements qui caractérisent notre système, dans le but de contraindre l'amplitude des fluctuations de la super-rotation de la graine. Ces oscillations semblent majoritairement engendrées par un écoulement cisaillant du noyau fluide qui ne porte pas, ou très peu, de moment cinétique. De plus, nous observons que le paramètre qui influence le plus les oscillations de rotation de la graine est l'intensité du couple gravitationnel entre la graine et le manteau. Ces observations permettent de contraindre à $0.04^{\circ} \mathrm{an}^{-1}$ l'amplitude des fluctuations décennales de la rotation différentielle de la graine, une valeur près de deux ordres de grandeur inférieure à celle estimée par l'étude sismologique menée par Tkalčić et al. (2013). 


\subsection{Typical standard deviations}

We here confront the time-dependent models developed in chapter 5 and tested against simple case-study simulations in chapter 6 to full convective numerical simulations of the geodynamo. To do so, we use the same simulations as in the long-term study of the coupled system of chapter 4 (Pichon et al., 2016), and listed in table 7.1. However, characteristic dimensions are the magnetic diffusion time scale of the core for the time, a typical angular velocity for dynamic components and the magnitude of the dipole for magnetic quantities, as adopted in the previous chapter. This first step toward the time-dependent analysis of complex simulations is performed with the standard deviations of the rotational dynamics components, which represents the mean amplitudes of the temporal fluctuations. Unless specified, the following quantities thus refer to dimensionless standard deviations. In order to base our analysis on already characterized parameters, the standard deviations of the angular velocities and shears are dimensioned by the time-averaged global shear in the fluid outer core $\left\langle\mathcal{C}_{\mathrm{f}}\right\rangle$ of each simulation. The electromagnetic torques scalings are then obtained with the parameter

$$
\mathcal{P}=\frac{\eta}{\left\langle\mathcal{C}_{\mathrm{f}}\right\rangle D^{2}},
$$

while the Lundquist number is defined as

$$
\mathcal{L} u=\frac{D\left\langle B_{\text {dip }}\right\rangle}{\eta_{\mathrm{c}} \sqrt{\mu_{0} \rho}},
$$

with $\left\langle B_{\text {dip }}\right\rangle$ the time-average value of the magnetic dipole field at the core-mantle boundary. As in the long-term study, we first analyze the response of the system to changes of the main control parameters. The first is the strength of the gravitational coupling embodied by the parameter $\zeta_{\eta}$ and the second is the lower mantle conductance $\Sigma$, whose expressions are respectively

$$
\zeta_{\eta}=\frac{\tau \Gamma}{\rho \eta D^{3}}, \quad \text { and } \quad \Sigma=\frac{\sigma_{\mathrm{m}} \Delta}{\sigma_{\mathrm{c}} D} .
$$

Figure 7.1 shows the standard deviations of the shear at the inner core boundary $\mathcal{C}_{\text {icb }}$, the shear in the fluid outer core $\mathcal{C}_{\mathrm{f}}$ as well as those of the inner core super-rotation $\mathcal{S}$ and the westward drift $\mathcal{D}$ as a function of the strength of the gravitational coupling between the mantle and the inner core. The system appears dominated by fluctuations of $\mathcal{C}_{\mathrm{f}}$ and $\mathcal{C}_{\text {icb }}$, while the amplitude of the westward drift fluctuations are nearly twice less. This figure also exhibits the drastic decrease of the inner core super-rotation as the strength of the gravitational coupling increases. As the time-averaged value of $\mathcal{S}$ is affected the same way, this demonstrates that the strength of the gravitational coupling is a dominant parameter that controls both the mean value and the amplitude of the fluctuations of the inner core differential rotation. However, contrary to the long-term state of the system, the standard deviations of the other three characteristic shears remains unaffected by changes of $\zeta_{\eta}$.

Figure 7.2 displays the standard deviations of the system as a function of the mantle 
conductance $\Sigma$, in simulations with a strong gravitational coupling, $\zeta_{\eta}=6.25 \times 10^{4}$, which is equivalent to $\zeta=0.75$ in the dimensionless system developed for the long-term analysis in chapter 4 , a value which is known to inhibit any long-term differential rotation of the inner core relative to the mantle. This value is shown to also strongly limit the amplitude of the fluctuations of the inner core differential rotation, while the other characteristic shears are weakly affected by changes on $\Sigma$. Figures 7.1 and 7.2 demonstrate that the main

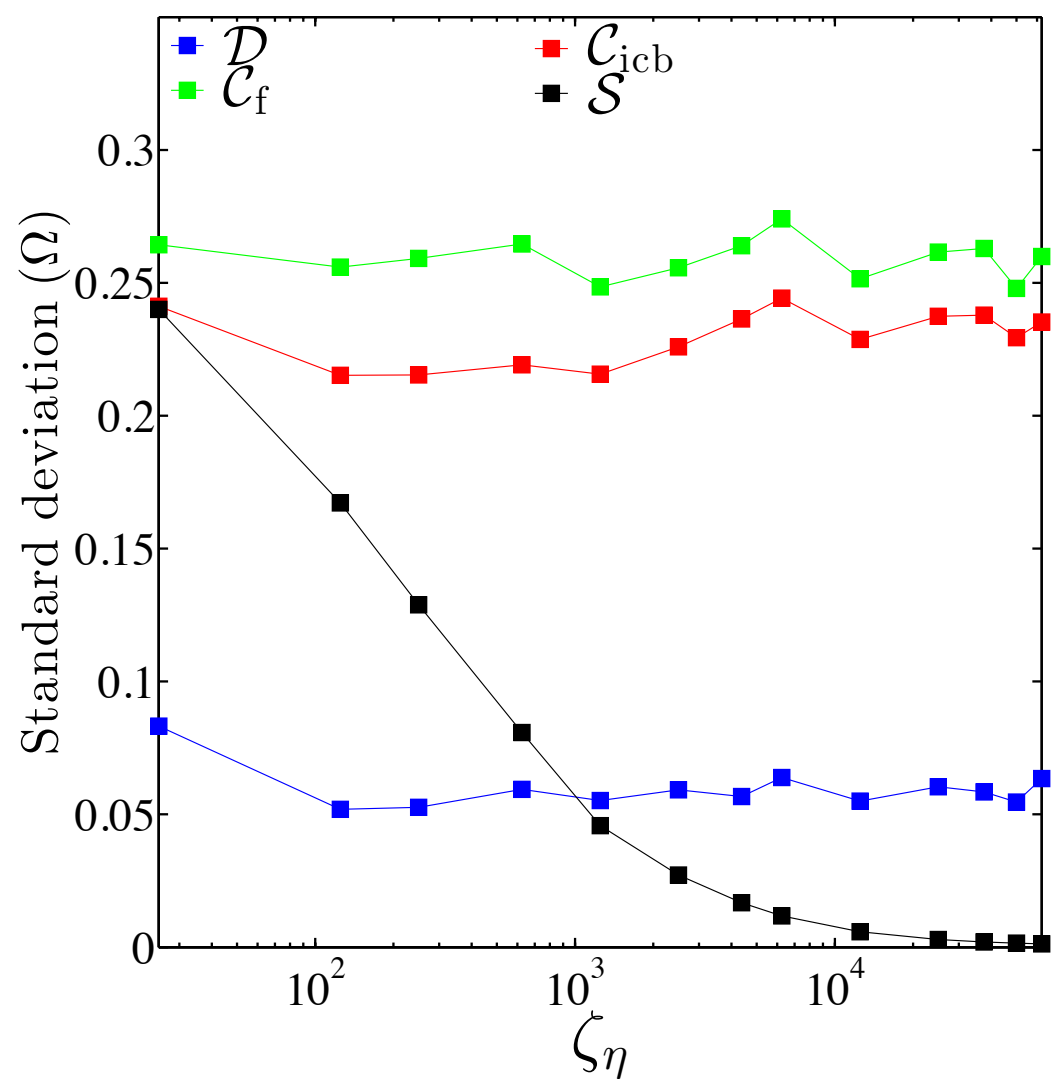

Figure 7.1: Standard deviations of $\mathcal{D}, \mathcal{C}_{\mathrm{f}}, \mathcal{C}_{\text {icb }}$ and $\mathcal{S}$ as functions of the strength of gravitational coupling $\zeta_{\eta}$ in a set of numerical simulations where $\Sigma=10^{-4}, \mathcal{R} a_{F}=2.7 \times 10^{-5}, E=3 \times 10^{-5}$ and $E_{\eta}=1.2 \times 10^{-5}$, see table 7.1 .

controlling factor of the inner core super-rotation is the strength of gravitational coupling, whose increase bounds the amplitudes of the fluctuations of $\mathcal{S}$ to remarkably small values.

\subsection{Electromagnetic torques}

This section confronts the time-dependent electromagnetic torque models expressed and tested in case-study simulations in the previous chapters to the outputs of the geodynamo simulations. Figures 7.3 and 7.4 display the agreement between the observed electromagnetic torques at respectively ICB and CMB and their respective models (black line), with slopes obtained in chapter 6 . Quantities $\Gamma_{\mathrm{ICB}}, \Gamma_{\mathrm{CMB}}, \mathcal{D}$ and $\mathcal{C}_{\text {icb }}$ refer to standard deviations while the $B_{\text {ri }}$ and $B_{\text {ro }}$ are the time-average r.m.s of the magnetic field at the inner core and 


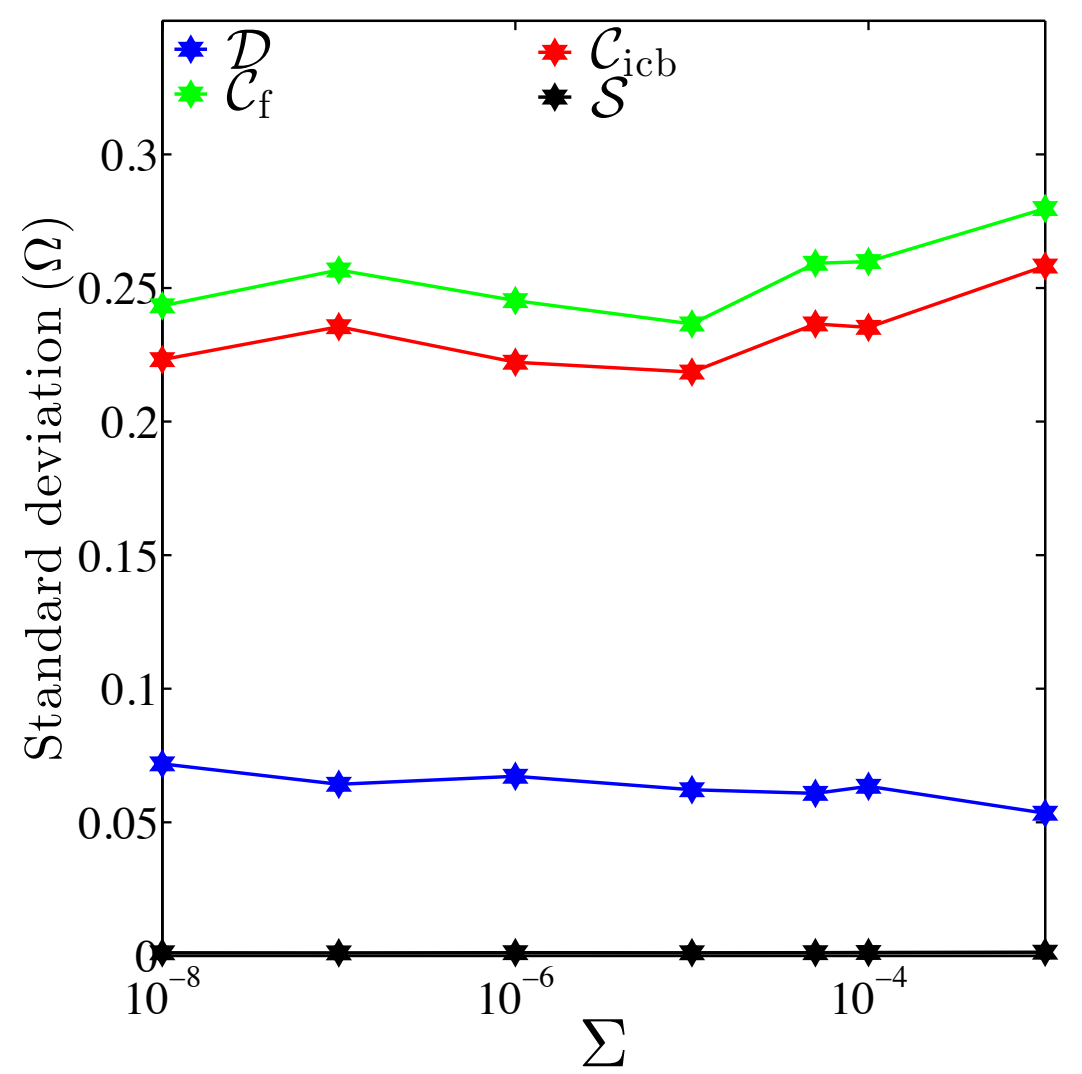

Figure 7.2: Standard deviations of $\mathcal{D}, \mathcal{C}_{\mathrm{f}}, \mathcal{C}_{\text {icb }}$ and $\mathcal{S}$ as functions of the mantle conductance $\Sigma$ in a set of numerical simulations where $\zeta_{\eta}=6.25 \times 10^{4}, \mathcal{R} a_{F}=2.7 \times 10^{-5}, E=3 \times 10^{-5}$ and $E_{\eta}=1.2 \times 10^{-5}$, see table 7.1 .

core-mantle boundary, respectively. Also, as the seismically inferred oscillations of the inner core differential rotations are decadal oscillations (Tkalčić et al., 2013), we use a 10 years (i.e. $\omega \tau_{\eta}=1.9 \times 10^{5}$ ) period to estimate the electromagnetic torque at the ICB. Please note that since the magnetic Reynolds number of most of our numerical simulations is similar to that of the Earth's core, similar results would be obtained if time were scaled according to the secular variation time scale as done for instance in Lhuillier et al. (2011). In both cases, simulations that depart from the order one scaling laws are simulations with low gravitational coupling $\left(\zeta_{\eta}<6 \times 10^{-2}\right)$ and/or Rayleigh numbers lower than the standard Rayleigh number $\mathcal{R} a_{F}=2.7 \times 10^{-5}$ used in the majority of the simulations. For this reason, we do not present scaling laws of the electromagnetic torque but only comparisons to the ideal cases. The departure between the above identified simulations and the model may be understood in several ways. First, the fluctuations of the inner core rotation of highest amplitude may be associated to very long period signals. In such a situation, the shear in the fluid outer core may have an impact on the scaling of the electromagnetic torque at the ICB, which is omitted here. However, it is difficult to estimate this impact since it may depends on the signal frequency. Moreover, the impact of $\mathcal{C}_{\mathrm{f}}$ in the long-term study was obtained when $\left\langle\Gamma_{\mathrm{ICB}}\right\rangle=\left\langle\Gamma_{\mathrm{G}}\right\rangle=0$, a constraint that is no longer valid when considering the amplitudes of the fluctuations. Second, the long-term expression of the gravitational torque 


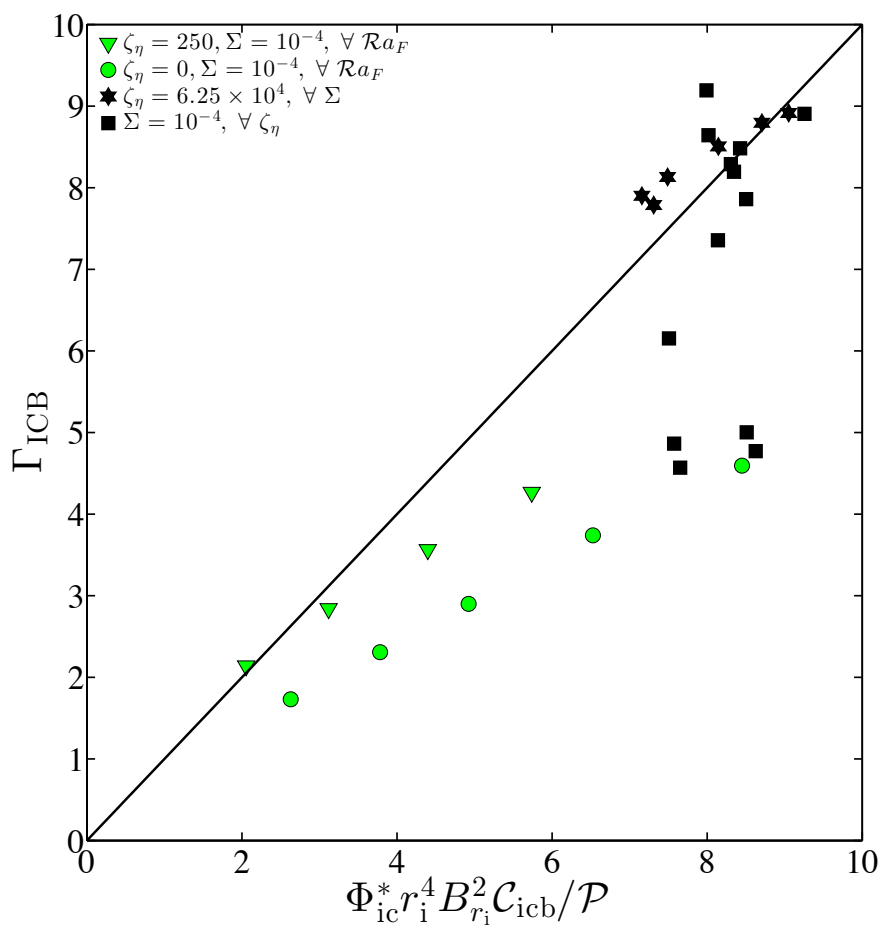

Figure 7.3: Comparison of the strength of electromagnetic torque between the geodynamo simulations outputs and the the model formulated in chapter 5 in terms of standard deviations. The slope is 1.14, as estimated from case-study simulations in chapter 6 .

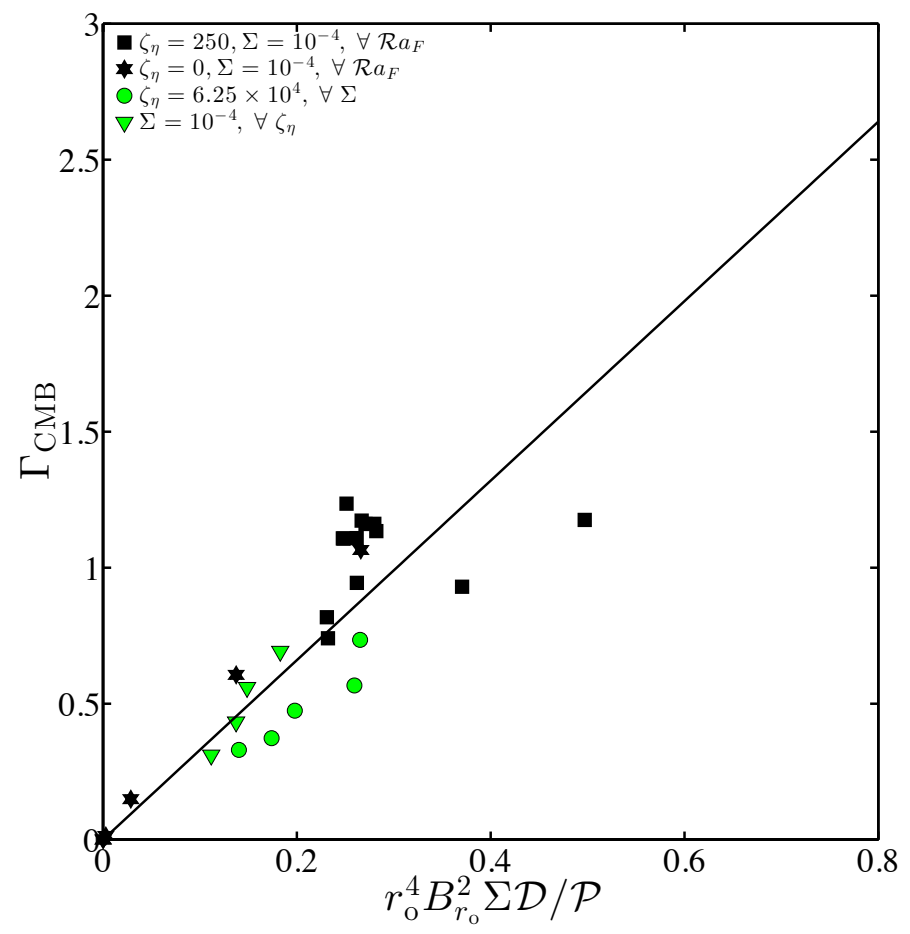

Figure 7.4: Comparison of the strength of electromagnetic torque between the geodynamo simulations outputs and the the model formulated in chapter 5 in terms of standard deviations. The slope is 3.33, as estimated from case-study simulations in chapter 6 . 


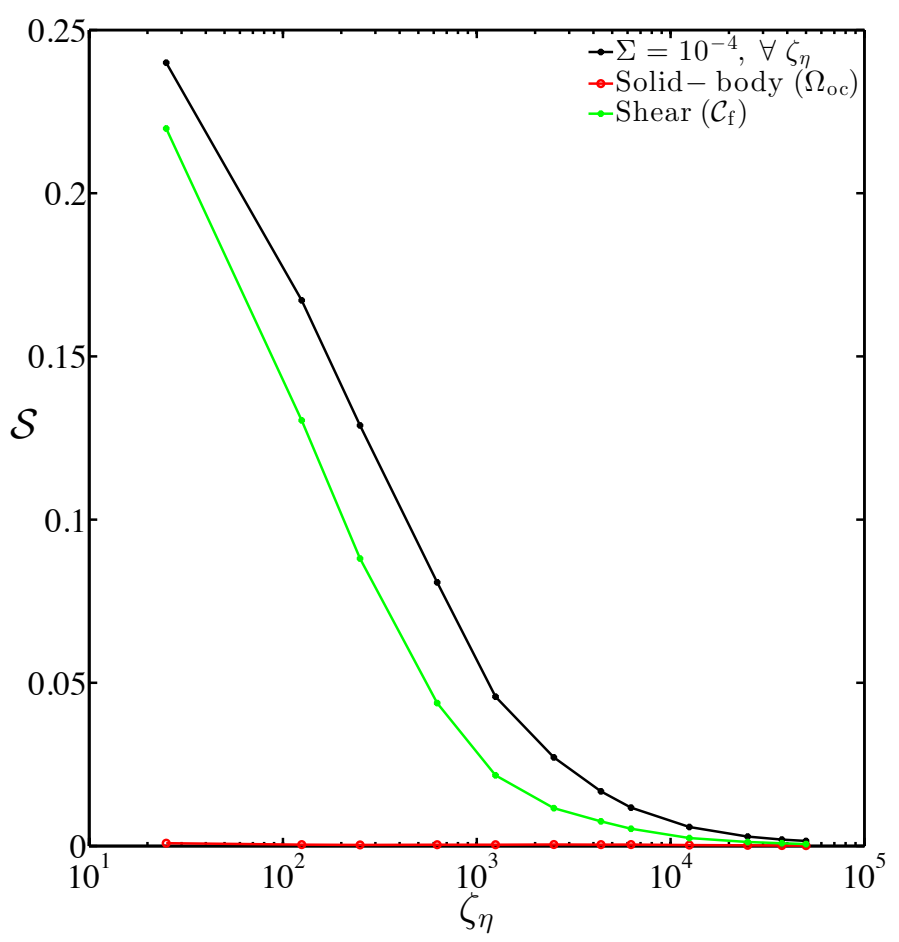

Figure 7.5: Comparison between the amplitude of the decadal fluctuations of the inner core super-rotation $\mathcal{S}$ in numerical simulations of the geodynamo (in black) and these inferred by the solid-body (red) and shear (green) ideal cases formulated in chapter 6, as a function of the strength of gravitational coupling $\zeta$.

may not perfectly represent the system's fluctuations for vanishing values of $\zeta_{\eta}$. Third, the already identified truncation errors (see section 4.5.1), affecting the torque balance and found to vanish when the radial resolution of the simulation is increased, may also induce similar errors in the standard deviations.

\subsection{A first estimate}

Although the previous section suggests that a scaling law for the standard deviations of the system is not straightforward to obtain, it is still feasible to compare the numerical results with the two ideal cases of chapter 6 . In the first case, the fluid outer core is modeled as an oscillating solid-body shell, so that the shear in the fluid outer core is zero. This formulation has the advantage of simply describing the angular momentum of the fluid outer core as $\mathrm{I}_{\mathrm{oc}} \Omega_{\mathrm{oc}}$, which may be retrieved by the monitoring of $\Omega_{\mathrm{ic}}$ and $\Omega_{\mathrm{m}}$ and the conservation of the angular momentum of the system. The second case is the opposite, the fluid outer core is modeled as a shear flow which, at any given time, bears no angular momentum. The shear in the fluid outer core is then maximum and the angular momentum balance is between the inner core and the mantle. Figure 7.5 present the comparisons between the inner core super-rotation $\mathcal{S}$ observed in the numerical simulations (in black) and the two ideal situations of a solid-body (red) and a shear (green) flow in the fluid outer 
core, in terms of standard deviations. As we identified the strength of the gravitational coupling as the main controlling factor, we thus display this comparison as a function of $\zeta_{\eta}$. Remarkably, the shear flows situation is very close to the observed $\mathcal{S}$, while the solid-body case is close to zero. This suggests that the amplitude of the decadal fluctuations of the inner core super-rotation is mostly governed by flows that bears no angular momentum. Then, though it is still difficult to obtain scaling laws, this similarity may be used to recover an estimate of the amplitude of the time-dependent inner core super-rotation. To do so, we refer to the discussion on the long-term state of the system developed in section 4.4. Given the experimentally inferred values of the inner core viscosity $\left(10^{15}-10^{18} \mathrm{~Pa} \mathrm{~s}\right.$, Gleason and Mao, 2013) and estimates of the strength of the gravitational coupling constant (Davies et al., 2014), we find that

$$
8.4 \times 10^{2}<\zeta_{\eta}<5.6 \times 10^{6}
$$

taking a mean density of the fluid outer core $\rho=11000 \mathrm{~kg} \mathrm{~m}^{-3}$ and a magnetic diffusivity based on the mineral physics experiments of Pozzo et al. (2012). In this range, we then observe that the dimensionless value of the inner core super-rotation is consistently lower than 0.05. Given our choice of characteristic dimensions, this reflects a situation where the amplitudes of the fluctuations of the inner core super-rotation represents less than $8 \%$ of the time-average global shear in the fluid outer core $\left\langle\mathcal{C}_{\mathrm{f}}\right\rangle$. Again referring to section 4.4, this time-averaged shear embodies the vigor of convection and is found to scale as the square root of the Rayleigh flux number $\mathcal{R} a_{F}$. A present-day estimate of this shear leads to $\left\langle\mathcal{C}_{\mathrm{f}}\right\rangle=0.42^{\circ} \mathrm{yr}^{-1}$, which in return constrains the decadal fluctuations of the inner core differential rotation to be lower than $0.04^{\circ} \mathrm{yr}^{-1}$. 


\begin{tabular}{|c|c|c|c|c|c|c|c|c|c|c|}
\hline $\mathcal{R} a_{F}$ & $\Sigma$ & $\zeta_{\eta}$ & $\mathcal{D}$ & $\mathcal{S}$ & $\mathcal{C}_{\mathrm{f}}$ & $\mathcal{C}_{\text {icb }}$ & $B_{\text {ri }}$ & $B_{\text {ro }}$ & $\mathcal{P}$ & $\mathcal{L} u$ \\
\hline $2.70 \times 10^{-5}$ & $1.00 \times 10^{-4}$ & 0 & $1.12 \times 10^{-1}$ & $2.71 \times 10^{-1}$ & $2.63 \times 10^{-1}$ & $2.48 \times 10^{-1}$ & $2.04 \times 10^{1}$ & 3.03 & $1.16 \times 10^{-3}$ & $8.80 \times 10^{1}$ \\
\hline $2.70 \times 10^{-5}$ & $1.00 \times 10^{-4}$ & $2.50 \times 10^{1}$ & $8.32 \times 10^{-2}$ & $2.40 \times 10^{-1}$ & $2.64 \times 10^{-1}$ & $2.41 \times 10^{-1}$ & $2.08 \times 10^{1}$ & 3.06 & $1.18 \times 10^{-3}$ & $8.55 \times 10^{1}$ \\
\hline $2.70 \times 10^{-5}$ & $1.00 \times 10^{-4}$ & $1.25 \times 10^{2}$ & $5.19 \times 10^{-2}$ & $1.67 \times 10^{-1}$ & $2.56 \times 10^{-1}$ & $2.15 \times 10^{-1}$ & $2.07 \times 10^{1}$ & 3.04 & $1.16 \times 10^{-3}$ & $8.88 \times 10^{1}$ \\
\hline $2.70 \times 10^{-5}$ & $1.00 \times 10^{-4}$ & $2.50 \times 10^{2}$ & $5.26 \times 10^{-2}$ & $1.29 \times 10^{-1}$ & $2.59 \times 10^{-1}$ & $2.15 \times 10^{-1}$ & $2.08 \times 10^{1}$ & 3.04 & $1.19 \times 10^{-3}$ & $8.84 \times 10^{1}$ \\
\hline $2.70 \times 10^{-5}$ & $1.00 \times 10^{-4}$ & $6.25 \times 10^{2}$ & $5.94 \times 10^{-2}$ & $8.08 \times 10^{-2}$ & $2.65 \times 10^{-1}$ & $2.19 \times 10^{-1}$ & $2.06 \times 10^{1}$ & 3.07 & $1.20 \times 10^{-3}$ & $8.68 \times 10^{1}$ \\
\hline $2.70 \times 10^{-5}$ & $1.00 \times 10^{-4}$ & $1.25 \times 10^{3}$ & $5.52 \times 10^{-2}$ & $4.57 \times 10^{-2}$ & $2.48 \times 10^{-1}$ & $2.16 \times 10^{-1}$ & $2.13 \times 10^{1}$ & 3.13 & $1.16 \times 10^{-3}$ & $8.32 \times 10^{1}$ \\
\hline $2.70 \times 10^{-5}$ & $1.00 \times 10^{-4}$ & $2.50 \times 10^{3}$ & $5.92 \times 10^{-2}$ & $2.72 \times 10^{-2}$ & $2.56 \times 10^{-1}$ & $2.26 \times 10^{-1}$ & $2.13 \times 10^{1}$ & 3.12 & $1.20 \times 10^{-3}$ & $8.31 \times 10^{1}$ \\
\hline $2.70 \times 10^{-5}$ & $1.00 \times 10^{-4}$ & $4.38 \times 10^{3}$ & $5.67 \times 10^{-2}$ & $1.67 \times 10^{-2}$ & $2.64 \times 10^{-1}$ & $2.36 \times 10^{-1}$ & $2.11 \times 10^{1}$ & 3.05 & $1.20 \times 10^{-3}$ & $8.83 \times 10^{1}$ \\
\hline $2.70 \times 10^{-5}$ & $1.00 \times 10^{-4}$ & $6.25 \times 10^{3}$ & $6.39 \times 10^{-2}$ & $1.18 \times 10^{-2}$ & $2.74 \times 10^{-1}$ & $2.44 \times 10^{-1}$ & $2.07 \times 10^{1}$ & 3.09 & $1.22 \times 10^{-3}$ & $8.60 \times 10^{1}$ \\
\hline $2.70 \times 10^{-5}$ & $1.00 \times 10^{-4}$ & $1.25 \times 10^{4}$ & $5.50 \times 10^{-2}$ & $5.82 \times 10^{-3}$ & $2.52 \times 10^{-1}$ & $2.29 \times 10^{-1}$ & $2.12 \times 10^{1}$ & 3.08 & $1.18 \times 10^{-3}$ & $8.52 \times 10^{1}$ \\
\hline $2.70 \times 10^{-5}$ & $1.00 \times 10^{-4}$ & $2.50 \times 10^{4}$ & $6.04 \times 10^{-2}$ & $2.89 \times 10^{-3}$ & $2.62 \times 10^{-1}$ & $2.37 \times 10^{-1}$ & $2.03 \times 10^{1}$ & 3.12 & $1.18 \times 10^{-3}$ & $8.42 \times 10^{1}$ \\
\hline $2.70 \times 10^{-5}$ & $1.00 \times 10^{-4}$ & $3.75 \times 10^{4}$ & $5.84 \times 10^{-2}$ & $1.96 \times 10^{-3}$ & $2.63 \times 10^{-1}$ & $2.38 \times 10^{-1}$ & $2.20 \times 10^{1}$ & 3.12 & $1.20 \times 10^{-3}$ & $8.29 \times 10^{1}$ \\
\hline $2.70 \times 10^{-5}$ & $1.00 \times 10^{-4}$ & $5.00 \times 10^{4}$ & $5.46 \times 10^{-2}$ & $1.54 \times 10^{-3}$ & $2.48 \times 10^{-1}$ & $2.29 \times 10^{-1}$ & $2.06 \times 10^{1}$ & 3.10 & $1.17 \times 10^{-3}$ & $8.46 \times 10^{1}$ \\
\hline $2.70 \times 10^{-5}$ & $1.00 \times 10^{-8}$ & $6.25 \times 10^{4}$ & $7.19 \times 10^{-2}$ & $1.14 \times 10^{-3}$ & $2.43 \times 10^{-1}$ & & $2.01 \times 10^{1}$ & 3.04 & $1.16 \times 10^{-3}$ & $8.70 \times 10^{1}$ \\
\hline $2.70 \times 10^{-5}$ & $1.00 \times 10^{-7}$ & $6.25 \times 10^{4}$ & $6.42 \times 10^{-2}$ & $1.14 \times 10^{-3}$ & $2.57 \times 10^{-1}$ & $2.35 \times 10^{-1}$ & $2.16 \times 10^{1}$ & 3.09 & $1.17 \times 10^{-3}$ & $8.27 \times 10^{1}$ \\
\hline $2.70 \times 10^{-5}$ & $1.00 \times 10^{-6}$ & $6.25 \times 10^{4}$ & $6.72 \times 10^{-2}$ & $1.18 \times 10^{-3}$ & $2.45 \times 10^{-1}$ & $2.22 \times 10^{-1}$ & $2.00 \times 10^{1}$ & 2.99 & $1.17 \times 10^{-3}$ & $9.01 \times 10^{1}$ \\
\hline $2.70 \times 10^{-5}$ & $1.00 \times 10^{-5}$ & $6.25 \times 10^{4}$ & & & & & & 3.06 & $1.14 \times 10^{-3}$ & \\
\hline $2.70 \times 10^{-5}$ & $5.00 \times 10^{-5}$ & $6.25 \times 10^{4}$ & $6.08 \times 10^{-2}$ & $1.19 \times 10^{-3}$ & $2.59 \times 10^{-1}$ & $2.37 \times 10^{-1}$ & $2.12 \times 10^{1}$ & 3.08 & $1.18 \times 10^{-3}$ & $8.44 \times 10^{1}$ \\
\hline $2.70 \times 10^{-5}$ & $1.00 \times 10^{-4}$ & $6.25 \times 10^{4}$ & $6.35 \times 10^{-2}$ & $1.26 \times 10^{-3}$ & $2.60 \times 10^{-1}$ & $2.35 \times 10^{-1}$ & $1.95 \times 10^{1}$ & 3.00 & $1.21 \times 10^{-3}$ & $9.07 \times 10^{1}$ \\
\hline $2.70 \times 10^{-5}$ & $1.00 \times 10^{-4}$ & 0 & $6.02 \times 10^{-2}$ & $2.52 \times 10^{-1}$ & $2.48 \times 10^{-1}$ & $2.43 \times 10^{-1}$ & $2.02 \times 10^{1}$ & 2.98 & $1.13 \times 10^{-3}$ & $9.09 \times 10^{1}$ \\
\hline $2.25 \times 10^{-5}$ & $1.00 \times 10^{-4}$ & 0 & $7.27 \times 10^{-2}$ & $2.65 \times 10^{-1}$ & $2.38 \times 10^{-1}$ & $2.39 \times 10^{-1}$ & $1.84 \times 10^{1}$ & 2.77 & $1.21 \times 10^{-3}$ & $9.89 \times 10^{1}$ \\
\hline $1.80 \times 10^{-5}$ & $1.00 \times 10^{-4}$ & 0 & $7.09 \times 10^{-2}$ & $2.83 \times 10^{-1}$ & $2.46 \times 10^{-1}$ & $2.45 \times 10^{-1}$ & $1.67 \times 10^{1}$ & 2.59 & $1.35 \times 10^{-3}$ & $1.08 \times 10^{2}$ \\
\hline $1.35 \times 10^{-5}$ & $1.00 \times 10^{-4}$ & 0 & & $3.15 \times 10^{-1}$ & $2.66 \times 10^{-1}$ & $2.63 \times 10^{-1}$ & $1.53 \times 10^{1}$ & 2.42 & $1.57 \times 10^{-3}$ & $1.14 \times 10^{2}$ \\
\hline $9.00 \times 10^{-6}$ & $1.00 \times 10^{-4}$ & 0 & $9.83 \times 10^{-2}$ & $3.61 \times 10^{-1}$ & $2.96 \times 10^{-1}$ & $2.84 \times 10^{-1}$ & $1.38 \times 10^{1}$ & 2.24 & $1.99 \times 10^{-3}$ & $1.18 \times 10^{2}$ \\
\hline $9.00 \times 10^{-6}$ & $1.00 \times 10^{-4}$ & $2.50 \times 10^{2}$ & $7.90 \times 10^{-2}$ & $1.98 \times 10^{-1}$ & $2.87 \times 10^{-1}$ & $2.23 \times 10^{-1}$ & $1.38 \times 10^{1}$ & 2.25 & $2.01 \times 10^{-3}$ & $1.17 \times 10^{2}$ \\
\hline $1.35 \times 10^{-5}$ & $1.00 \times 10^{-4}$ & $2.50 \times 10^{2}$ & $6.20 \times 10^{-2}$ & $1.66 \times 10^{-1}$ & $2.53 \times 10^{-1}$ & $2.03 \times 10^{-1}$ & $1.57 \times 10^{1}$ & 2.48 & $1.56 \times 10^{-3}$ & $1.08 \times 10^{2}$ \\
\hline $1.80 \times 10^{-5}$ & $1.00 \times 10^{-4}$ & $2.50 \times 10^{2}$ & $5.05 \times 10^{-2}$ & $1.49 \times 10^{-1}$ & $2.44 \times 10^{-1}$ & $2.03 \times 10^{-1}$ & $1.74 \times 10^{1}$ & 2.66 & $1.35 \times 10^{-3}$ & $9.97 \times 10^{1}$ \\
\hline $2.25 \times 10^{-5}$ & $1.00 \times 10^{-4}$ & $2.50 \times 10^{2}$ & $4.94 \times 10^{-2}$ & $1.36 \times 10^{-1}$ & $2.42 \times 10^{-1}$ & $2.03 \times 10^{-1}$ & $1.91 \times 10^{1}$ & 2.87 & $1.25 \times 10^{-3}$ & $9.29 \times 10^{1}$ \\
\hline
\end{tabular}

Table 7.1: Parameters and outputs (standard deviations except for $B_{\mathrm{ri}}$ and $B_{\mathrm{ro}}$ ) of the numerical simulations used in this chapter. All runs were performed with $E=E_{\kappa}=3 \times 10^{-5}$ and $E_{\eta}=1.2 \times 10^{-5}$. 


\section{Conclusions and perspectives}

This work evidences the link between two salient features of the rotational dynamics of the Earth. The first is the geomagnetic westward drift, the most documented component of the secular variation of the Earth's magnetic field. Four centuries of geomagnetic field measurements indeed constrains the westward drift as being located close to the equator of the Atlantic hemisphere, with an average velocity of 17 kilometers per year. The second is the so-called inner core super-rotation, a differential rotation of the inner core relative to the mantle, which is much less constrained at present.

In the long-term, the link between the two is embodied by the global, time-averaged, shear in the fluid outer core. Remarkably, the amplitude of the latter is independent of the state of coupling between the inner core, the fluid outer core and the mantle.It is however proved to be a function of the vigor of convection in the fluid outer core, and therefore scales with the Rayleigh number. A determined portion of this shear is available at the inner core boundary and/or at the core-mantle boundary and its distribution is governed by the relative amplitudes of the direct electromagnetic torques at the fluid core boundaries and the gravitational torque between the inner core and the mantle. Given the many uncertainties on deep Earth parameters, an estimate of the long-term coupling state of the system is nevertheless not straightforward. Our first conclusion then lies in a present day estimate of the global shear available in the fluid outer core, which is found to be close to the mean westward drift velocity in the last four hundred years. Thus, the time average state is dominated by the westward drift, which in turns prevails any long-term differential rotation of the inner core, relative to the mantle. In the case where the lower mantle conductance is of $10^{8} \mathrm{~S}$, the prefered long-term distribution of the shear toward the core-mantle boundary and the westward drift then provides a constraint on the viscosity of the inner core, which is necessarily larger than $2 \times 10^{17} \mathrm{~Pa}$ s.

This work also provides the basis toward a full analysis of the time-dependent behavior of the system. We committed ourselves to clearly demonstrate the expressions the timedependant electromagnetic torque at the fluid core boundaries and determine their validity domain. These models were tested in case-study numerical simulations for which the time- 
dependent angular momentum balance of the system has a simple solution: a solid-body rotation of the fluid outer core and a shear-flow carrying no angular momentum. From our improved understanding of the time-dependent system follows a preliminary application to fully-convective simulations of the geodynamo, leading to two main observations. First, the amplitude of the oscillations of the inner core differential rotation is mainly impacted by the strength of the gravitational torque that links it to the mantle. Second, as the amplitude of the fluctuations as a function of the strength of the gravitational torque highly resembles that predicted by the ideal shear flow case, the oscillations of the inner core rotation should be mainly driven by flows that bears little or no angular momentum. Given the range of possible values of the strength of gravitational coupling, we then found that the amplitudes of the decadal oscillations of the inner core differential rotation should not exceed $0.04^{\circ} \mathrm{yr}^{-1}$.

Although they are bounded to the limits of geodynamo simulations and dependent on some poorly constrained deep Earth parameters, our conclusions highly contrast with the most recent seismological study of Tkalčić et al. (2013) suggesting a time-average inner core super-rotation of $0.25-0.48^{\circ} \mathrm{yr}^{-1}$ accompanied by decadal fluctuations on the order of $1^{\circ} \mathrm{yr}^{-1}$. Instead, we propose that the inner core super-rotation is a vanishing feature of the rotational dynamics of the long-term system, which may endure decadal fluctuations around a zero mean on the order of a few hundredths of degree per year.

A natural perspective of this work is to not restrict the time-dependent analysis of the system to its standard deviations. The complete application to the developed models and simplified solutions should indeed include the frequency spectrum of each component of the rotational dynamics of the system. The idea is then to reproduce the work of the long-term study: propose a scaling of the electromagnetic torques and establish links between the rotational components. This should in theory leads to a description of the oscillating system, frequency by frequency, and ultimately constrain the amplitudes of the different harmonic components of the inner core differential rotation. 


\section{Appendices}

\section{A.1 Interface conditions}

\section{Interface conditions on $\mathrm{E}$ and $\mathrm{B}$}

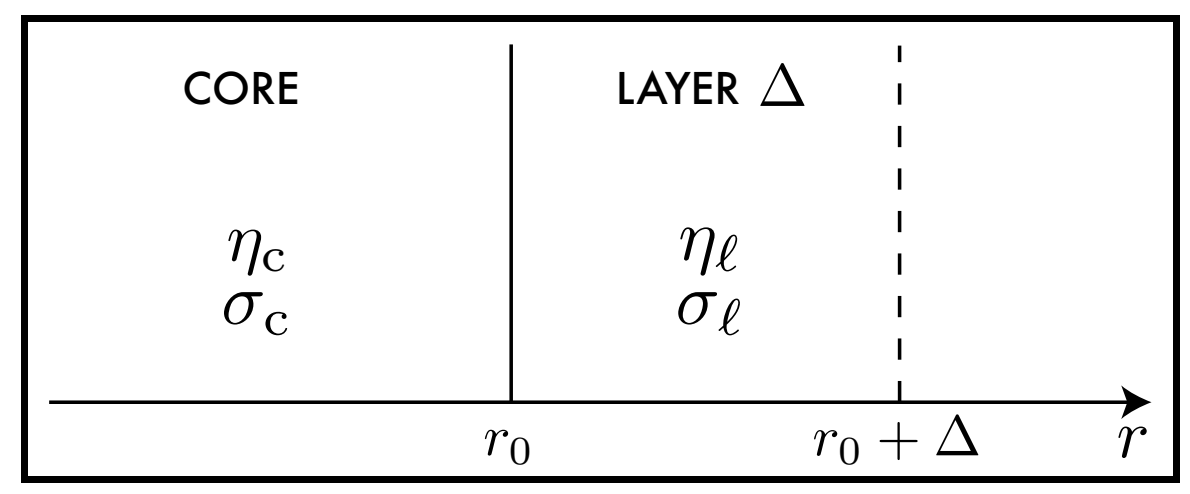

Figure A.1

This part is dedicated to the formulation of the interface conditions on the magnetic $\mathbf{B}\left(B_{\mathrm{r}}, B_{\theta}, B_{\varphi}\right)$ and the electric $\mathbf{E}\left(E_{\mathrm{r}}, E_{\theta}, E_{\varphi}\right)$ fields at the fluid core boundaries, considering the existence of an electrically conducting layer, of thickness $\Delta$ at the base of the mantle. In order to analyze the crossing of a magnetic or electric field coming from the core, that will diffuses into the inner core or into the conducting lower mantle, we generalize the situation as depicted by figure A.1. We study the interface conditions on a field that comes from the core, of electrical conductivity $\sigma_{\mathrm{c}}$ that will diffuse into a layer of thickness $\Delta$, located in $r_{0}$, with electrical conductivity $\sigma_{\ell}$. Within this framework, the following interface conditions hold at both ICB and CMB. These boundary conditions directly flow from the Maxwell's equations and express

1. The continuity of the radial magnetic field $\left(B_{\mathrm{r}}\right)$,

$$
\left.\mathbf{r} \cdot \mathbf{B}\right|_{\text {core }}=\left.\mathbf{r} \cdot \mathbf{B}\right|_{\text {layer }}
$$


with $\mathbf{r}$ the radial vector directed from the core toward the layer.

2. The discontinuity of the tangential magnetic field $\left(B_{\theta}, B_{\varphi}\right)$,

$$
\mathbf{r} \times\left.\mathbf{B}\right|_{\text {core }}=\mathbf{r} \times\left.\mathbf{B}\right|_{\text {layer }}-\mathbf{j}_{\mathbf{s}} \mu_{0}
$$

with $\mathbf{j}_{\mathbf{s}}$ the surface current density between the core and the layer and $\mu_{0}$ the magnetic permeability of free space.

3. The discontinuity of the radial electric field $\left(E_{\mathrm{r}}\right)$,

$$
\left.\mathbf{r} \cdot \mathbf{E}\right|_{\text {core }}=\left.\mathbf{r} \cdot \mathbf{E}\right|_{\text {layer }}-\frac{\rho_{s}}{\epsilon_{0}}
$$

with $\rho_{s}$ the surface charge density and the $\epsilon_{0}$ the permittivity of free space.

4. The continuity of the tangential electric field $\left(E_{\theta}, E_{\varphi}\right)$,

$$
\mathbf{r} \times\left.\mathbf{E}\right|_{\text {core }}=\mathbf{r} \times\left.\mathbf{E}\right|_{\text {layer }}
$$

In the case where there are no surface charge density $\left(\rho_{s}=0\right)$ and no surface currents $\left(j_{s}=0\right)$ between the two medium, these interface conditions simply express the continuity of the radial and tangential components of both magnetic and electric fields like

$$
\left.\mathbf{B}\right|_{\text {core }}=\left.\mathbf{B}\right|_{\text {layer }}
$$

and

$$
\left.\mathbf{E}\right|_{\text {core }}=\left.\mathbf{E}\right|_{\text {layer }}
$$

\section{Interface Conditions on toroidal and poloidal scalars}

As the vector fields are decomposed in toroidal and poloidal contributions, including the numerical implementation, it is convenient to explicit the interface conditions in terms of toroidal and poloidal scalars. We recall that the decomposition is

$$
\mathbf{B}=\boldsymbol{\nabla} \times \boldsymbol{\nabla} \times\left(\mathbf{r} B_{\mathrm{p}}\right)+\nabla \times\left(\mathbf{r} B_{\mathrm{t}}\right)
$$


such that the components of $\mathbf{B},\left(B_{\mathrm{r}}, B_{\theta}, B_{\varphi}\right)$, can be expressed as

$$
\left\{\begin{array}{r}
\frac{1}{r} L_{2}\left(B_{\mathrm{p}}\right) \\
\frac{\partial}{\partial \theta}\left(\frac{1}{r} \frac{\partial}{\partial r}\left(r B_{\mathrm{p}}\right)\right)+\frac{1}{\sin \theta} \frac{\partial B_{\mathrm{t}}}{\partial \varphi} \\
\frac{1}{\sin \theta} \frac{\partial}{\partial \varphi}\left(\frac{1}{r} \frac{\partial}{\partial r}\left(r B_{\mathrm{p}}\right)\right)-\frac{\partial B_{\mathrm{t}}}{\partial \theta}
\end{array}\right.
$$

As demonstrated by Backus (1986), the same decomposition can also be written

$$
\mathbf{B}=-\mathbf{r} \nabla^{2} B_{\mathrm{p}}+\boldsymbol{\nabla}\left(\frac{\partial}{\partial r}\left(r B_{\mathrm{p}}\right)\right)-\mathbf{r} \times \nabla B_{\mathrm{t}}
$$

the poloidal part of equation (A.7) being

$$
\boldsymbol{\nabla} \times \boldsymbol{\nabla} \times\left(\mathbf{r} B_{\mathrm{p}}\right)=-\mathbf{r} \Delta B_{\mathrm{p}}+\boldsymbol{\nabla}\left(\frac{\partial}{\partial r}\left(r B_{\mathrm{p}}\right)\right)
$$

and the toroidal part

$$
\nabla \times\left(\mathbf{r} B_{\mathrm{t}}\right)=-\mathbf{r} \times \nabla B_{\mathrm{t}}
$$

\section{Continuity of toroidal field}

The continuity of the magnetic field $\mathbf{B}$ across the interface necessarily involves the continuity the orthogonal poloidal and toroidal parts of the decomposition, leading to

$$
\mathbf{r} \times\left.\nabla B_{\mathrm{t}}\right|_{\text {core }}=\mathbf{r} \times\left.\nabla B_{\mathrm{t}}\right|_{\text {layer }}
$$

This in turns can be written

and

$$
\left.\frac{1}{\sin \theta} \frac{\partial B_{\mathrm{t}}}{\partial \varphi}\right|_{\text {core }}=\left.\frac{1}{\sin \theta} \frac{\partial B_{\mathrm{t}}}{\partial \varphi}\right|_{\text {layer }}
$$

$$
\left.\frac{\partial B_{\mathrm{t}}}{\partial \theta}\right|_{\text {core }}=\left.\frac{\partial B_{\mathrm{t}}}{\partial \theta}\right|_{\text {layer }}
$$

proving the continuity of the toroidal magnetic field $B_{\mathrm{t}}$ across the boundary.

\section{Continuity of poloidal field and its first radial derivative}

The same argument can be used to analyze the continuity of the poloidal magnetic field. We have

$$
\left[-\mathbf{r} \nabla^{2} B_{\mathrm{p}}+\boldsymbol{\nabla}\left(\frac{\partial}{\partial r}\left(r B_{\mathrm{p}}\right)\right)\right]_{\text {core }}=\left[-\mathbf{r} \boldsymbol{\Delta} B_{\mathrm{p}}+\boldsymbol{\nabla}\left(\frac{\partial}{\partial r}\left(r B_{\mathrm{p}}\right)\right)\right]_{\text {layer }}
$$


which can also be written

$$
\left[-\mathbf{r} \nabla^{2} B_{\mathrm{p}}+\nabla\left(\frac{\partial}{\partial r}\left(r B_{\mathrm{p}}\right)\right)\right]_{\text {core }}^{\text {layer }}=0
$$

in order to lighten the notation. If we decompose the laplacian $\left(\nabla^{2}\right)$ and the gradient $(\boldsymbol{\nabla})$ parts of the equation into their radial and horizontal components this gives

$$
\left[-\mathbf{r}\left(\frac{1}{r^{2}} \frac{\partial}{\partial r}\left(r^{2} \frac{B_{\mathrm{p}}}{\partial r}\right)+\frac{1}{r^{2}} L_{2} B_{\mathrm{p}}\right)+\frac{1}{r} \frac{\partial}{\partial r}\left(\frac{\partial}{\partial r}\left(r B_{\mathrm{p}}\right)\right) \mathbf{r}+\frac{1}{r} \nabla_{H}\left(\frac{\partial}{\partial r}\left(r B_{\mathrm{p}}\right)\right)\right]_{\text {core }}^{\text {layer }}=0
$$

with $\nabla_{H}$ the horizontal gradient. This leads to

$$
\left[-\frac{1}{r^{2}}\left(L_{2} B_{\mathrm{p}}\right) \mathbf{r}+\frac{1}{r} \nabla_{H}\left(\frac{\partial}{\partial r}\left(r B_{\mathrm{p}}\right)\right)\right]_{\text {core }}^{\text {layer }}=0
$$

As the left and right parts of equation (A.18) are orthogonal, this can be separate into two independent interface conditions. The left part involves that

$$
\left.L_{2} B_{\mathrm{p}}\right|_{\text {core }}=\left.L_{2} B_{\mathrm{p}}\right|_{\text {layer }}
$$

and proves the continuity of the poloidal part of the magnetic field across the boundary, while the right part involves that

$$
\left.\nabla_{H}\left(\frac{\partial}{\partial r}\left(r B_{\mathrm{p}}\right)\right)\right|_{\text {core }}=\left.\nabla_{H}\left(\frac{\partial}{\partial r}\left(r B_{\mathrm{p}}\right)\right)\right|_{\text {layer }}
$$

which is equivalent to

$$
\left.\frac{\partial B_{\mathrm{p}}}{\partial r}\right|_{\text {core }}=\left.\frac{\partial B_{\mathrm{p}}}{\partial r}\right|_{\text {layer }},
$$

proving the continuity of the first radial derivative of the poloidal part of the magnetic field.

\section{Discontinuity of the radial derivative of toroidal field}

The discontinuity of the first radial derivative of the toroidal magnetic field comes from the presence of an advective source term in Ohm's law in a medium where the velocity field is $\mathbf{u}$, with components $\left(u_{\mathrm{r}}, u_{\theta}, u_{\varphi}\right)$ in spherical coordinates. We start from the continuity of the tangential electric field across the interface,

using Ohm's law

$$
\mathbf{r} \times\left.\mathbf{E}\right|_{\text {core }}=\mathbf{r} \times\left.\mathbf{E}\right|_{\text {layer }}
$$

$$
\mathbf{E}=\frac{\mathbf{J}}{\sigma}-\mathbf{u} \times \mathbf{B}
$$


we have

$$
\mathbf{r} \times \frac{\mathbf{J}}{\sigma_{\mathrm{c}}}-\mathbf{r} \times \mathbf{u} \times\left.\mathbf{B}\right|_{\text {core }}=\mathbf{r} \times\left.\frac{\mathbf{J}}{\sigma_{\ell}}\right|_{\text {layer }}
$$

Applying Ampère's equation $\left(\mu_{0} \mathbf{J}=\boldsymbol{\nabla} \times \mathbf{B}\right)$ this eventually gives

$$
\eta_{\mathrm{c}} \mathbf{r} \times \boldsymbol{\nabla} \times \mathbf{B}-\mathbf{r} \times \mathbf{u} \times\left.\mathbf{B}\right|_{\text {core }}=\eta_{\ell} \mathbf{r} \times \nabla \times\left.\mathbf{B}\right|_{\text {layer }},
$$

with $\eta_{\mathrm{c}}=1 / \sigma_{\mathrm{c}} \mu_{0}$ and $\eta_{\ell}=1 / \sigma_{\ell} \mu_{0}$ the magnetic diffusivities of the core and the layer. As demonstrated by Holme (1998), if we apply the operator $\boldsymbol{\nabla} \cdot \mathbf{r} \times$ to equation (A.25), we have

$$
\eta \nabla \cdot \mathbf{r} \times \mathbf{r} \times \nabla \times \mathbf{B}=\eta \nabla \cdot(\mathbf{r}(\mathbf{r} \cdot \boldsymbol{\nabla} \times \mathbf{B})-\nabla \times \mathbf{B})
$$

for any vector $\mathbf{V}$, there is

$$
\nabla \cdot \nabla \times \mathrm{V}=0
$$

so that,

$$
\eta \nabla \cdot \mathbf{r} \times \mathbf{r} \times \boldsymbol{\nabla} \times \mathbf{B}=\frac{\eta}{r^{2}} \frac{\partial}{\partial r}\left(r^{3} \mathbf{r} \cdot \boldsymbol{\nabla} \times \mathbf{B}\right)
$$

in $r=r_{0}$,

$$
=r_{0} \eta_{\mathrm{c}} \frac{\partial}{\partial r}\left(L_{2} B_{\mathrm{t}}\right) \text {. }
$$

Finally, as $\boldsymbol{\nabla} \cdot \mathbf{r} \times \equiv-\mathbf{r} \cdot \boldsymbol{\nabla} \times$, the conservation of the tangential electric field across the interface thus becomes

$$
r_{0} \eta_{\mathrm{c}} \frac{\partial}{\partial r}\left(L_{2} B_{\mathrm{t}}\right)+\mathbf{r} \cdot \boldsymbol{\nabla}_{H} \times \mathbf{u} \times\left.\mathbf{B}\right|_{\text {core }}=\left.r_{0} \eta_{\ell} \frac{\partial}{\partial r}\left(L_{2} B_{\mathrm{t}}\right)\right|_{\text {layer }},
$$

expressing the discontinuity of the first radial derivative of the toroidal magnetic field.

\section{About the second radial derivative of the poloidal field}

In order to obtain insights on the continuity of the second radial derivative of the poloidal field coming from the core, we restart from the continuity of the electric field $\mathbf{E}$,

$$
\left.\mathbf{E}\right|_{\text {core }}=\left.\mathbf{E}\right|_{\text {layer }}
$$

As in the previous section, we use alternatively Ohm's and Ampère's laws to obtain

$$
\eta_{\mathrm{c}} \boldsymbol{\nabla} \times \mathbf{B}-\mathbf{u} \times\left.\mathbf{B}\right|_{\text {core }}=\eta_{\ell} \boldsymbol{\nabla} \times\left.\mathbf{B}\right|_{\text {layer }},
$$

to which we apply the operator $\mathbf{r} \cdot \nabla \times$, giving

$$
\eta_{\mathrm{c}} \mathbf{r} \cdot \boldsymbol{\nabla} \times \boldsymbol{\nabla} \times \mathbf{B}-\mathbf{r} \cdot \boldsymbol{\nabla} \times \mathbf{u} \times\left.\mathbf{B}\right|_{\text {core }}=\eta_{\ell} \mathbf{r} \cdot \nabla \times \nabla \times\left.\mathbf{B}\right|_{\text {layer }} .
$$


For any vector $\mathbf{V}$, we have

$$
\nabla \times \nabla \times \mathbf{V}=-\nabla \times \nabla \times\left(\mathrm{r} \Delta V_{\mathrm{p}}\right)+\nabla \times\left(\mathrm{r} \Delta V_{\mathrm{t}}\right)
$$

as

$$
\mathbf{r} \cdot \boldsymbol{\nabla} \times\left(\mathbf{r} \Delta V_{\mathrm{t}}\right)=0
$$

we have

$$
\mathbf{r} \cdot \nabla \times \nabla \times \mathbf{V}=-\mathbf{r} \cdot \nabla \times \nabla \times\left(\mathbf{r} \Delta V_{\mathrm{p}}\right)
$$

Decomposing the vectorial laplacian of $\mathbf{B},(\boldsymbol{\Delta} \mathbf{B})$, into toroidal and poloidal components like,

$$
\Delta \mathbf{B}=\nabla \times \nabla \times\left(\mathrm{r} \Delta B_{\mathrm{p}}\right)+\nabla \times\left(\mathrm{r} \Delta B_{\mathrm{t}}\right)
$$

leads to the conclusion that $\mathbf{r} \cdot \boldsymbol{\nabla} \times \boldsymbol{\nabla} \times \mathbf{B}$ is the radial component of this decomposition, such that

$$
\mathbf{r} \cdot \boldsymbol{\nabla} \times \boldsymbol{\nabla} \times \mathbf{B}=-r \times\left(\frac{1}{r} L_{2} \Delta B_{\mathrm{p}}\right)=-L_{2} \Delta B_{\mathrm{p}} .
$$

Eventually, the continuity of the electric field leads to

$$
\eta_{\mathrm{c}} L_{2} \Delta B_{\mathrm{p}}+\mathbf{r} \cdot \nabla \times \mathbf{u} \times\left.\mathbf{B}\right|_{\text {core }}=\left.\eta_{\ell} L_{2} \Delta B_{\mathrm{p}}\right|_{\text {layer }} .
$$

Equation (A.40) provides information on the laplacian of the poloidal field, and thus on its the second radial derivative, which can be continuous across the boundary if the condition

$$
\mathbf{r} \cdot \boldsymbol{\nabla} \times \mathbf{u} \times \mathbf{B}=0
$$

is achieved. In order to obtain further insights on this condition, we develop here this vectorial product considering a non-penetration condition at the interface,

$$
\left.u_{\mathrm{r}}\right|_{r_{0}}=0
$$

and under the assumption that the velocity field is mainly azimuthal, i.e. $u_{\theta} \ll u_{\varphi}$, we then have

$$
\mathbf{u}\left(\begin{array}{c}
0 \\
0 \\
u_{\varphi}
\end{array}\right) \times \mathbf{B}\left(\begin{array}{c}
B_{\mathrm{r}} \\
B_{\theta} \\
B_{\varphi}
\end{array}\right)=\left(\begin{array}{c}
-B_{\theta} u_{\varphi} \\
B_{\mathrm{r}} u_{\varphi} \\
0
\end{array}\right)
$$

and taking the rotational of this product gives

$$
\boldsymbol{\nabla}\left(\begin{array}{c}
\frac{\partial}{\partial r} \\
\frac{1}{r} \frac{\partial}{\partial \theta} \\
\frac{1}{r \sin \theta} \frac{\partial}{\partial \varphi}
\end{array}\right) \times \mathbf{u} \times \mathbf{B}=\left(\begin{array}{c}
\frac{1}{r \sin \theta} \frac{\partial B_{\mathrm{r}} u_{\varphi}}{\partial \varphi} \\
-\frac{1}{r \sin \theta} \frac{\partial B_{\theta} u_{\varphi}}{\partial \varphi} \\
\frac{1}{r \sin \theta} \frac{\partial r B_{\mathrm{r}} u_{\varphi}}{\partial r}+\frac{1}{r} \frac{\partial B_{\theta} u_{\varphi}}{\partial \theta}
\end{array}\right)
$$


Ultimately, equation (A.40) can now be written

$$
\eta_{\mathrm{c}} L_{2} \Delta B_{\mathrm{p}}+\left.\frac{1}{\sin \theta} \frac{\partial B_{\mathrm{r}} u_{\varphi}}{\partial \varphi}\right|_{\text {core }}=\left.\eta_{\ell} L_{2} \Delta B_{\mathrm{p}}\right|_{\text {layer }}
$$

providing the requirement that,

$$
\frac{1}{\sin \theta} \frac{\partial B_{\mathrm{r}} u_{\varphi}}{\partial \varphi}=0
$$

for the second radial derivative of the poloidal field to be continuous. In others words, we have

$$
\left.\eta_{\mathrm{c}} \frac{\partial^{2} B_{\mathrm{p}}}{\partial r^{2}}\right|_{\text {core }}=\left.\eta_{\ell} \frac{\partial^{2} B_{\mathrm{p}}}{\partial r^{2}}\right|_{\text {layer }},
$$

if both the radial magnetic field $B_{\mathrm{r}}$ and the azimuthal velocity $u_{\varphi}$ are invariant along the azimuthal direction, which should be the case if the magnetic field mainly embodied by the axial dipole and if the velocity field is essentially axisymmetric. This proves the strict continuity of the second radial derivative of the poloidal part of the magnetic field if the two media have the same magnetic diffusivity.

\section{Outlines}

To sum up, the continuity of the electrical field $\mathbf{E}$ and the magnetic field $\mathbf{B}$ imply diffusing from the fluid core into the inner core or an electrically conducting layer, imply the continuity of:

- the toroidal magnetic field $B_{\mathrm{t}}$,

- the poloidal magnetic field $B_{\mathrm{p}}$,

- the first radial derivative of the poloidal field $\frac{\partial B_{\mathrm{p}}}{\partial r}$.

Moreover, we showed that the first radial derivative of the toroidal field was discontinuous, because of the velocity jump between the core and the diffusing layer, i.e $\frac{\partial B_{\mathrm{t}}}{\partial r}$ is discontinuous. A similar conclusion holds for the second radial derivative of the poloidal magnetic field, that may be continuous across the boundary if the radial field $B_{\mathrm{r}}$ and the azimuthal velocity $u_{\varphi}$ are constants along the azimuthal direction, as prescribed by equation (A.45).

\section{A.2 Time-dependent Electromagnetic torques}

\section{Diffusion of a perturbation of the magnetic field}

\section{Mantle-side: Constant conductivity}

The diffusion of the toroidal scalar of the magnetic field into the conducting part of the mantle is described by

$$
X=A e^{\left(\frac{1-i}{\delta_{\mathrm{m}}}\right) r}+B e^{-\left(\frac{1-i}{\delta_{\mathrm{m}}}\right) r}
$$


which is subject to the following boundary conditions,

$$
\begin{aligned}
X\left(r_{\mathrm{o}}\right) & =\bar{X}, \\
X\left(r_{\mathrm{o}}+\Delta\right) & =0 .
\end{aligned}
$$

Applying the first boundary condition to equation (A.48) leads to

$$
\bar{X}=A e^{\left(\frac{1-i}{\delta_{\mathrm{m}}}\right) r_{\mathrm{o}}}+B e^{-\left(\frac{1-i}{\delta_{\mathrm{m}}}\right) r_{\mathrm{o}}}
$$

while condition (A.50) leads to

$$
0=A e^{\left(\frac{1-i}{\delta_{\mathrm{m}}}\right)\left(r_{\mathrm{o}}+\Delta\right)}+B e^{-\left(\frac{1-i}{\delta_{\mathrm{m}}}\right)\left(r_{\mathrm{o}}+\Delta\right)}
$$

such that

$$
A=-B e^{-2\left(\frac{1-i}{\delta_{\mathrm{m}}}\right)\left(r_{\mathrm{o}}+\Delta\right)}
$$

Introducing this expression of $A$ into (A.51) finally yields

$$
\begin{aligned}
& \bar{X}=-B e^{-2\left(\frac{1-i}{\delta_{\mathrm{m}}}\right)\left(r_{\mathrm{o}}+\Delta\right)} \times e^{\left(\frac{1-i}{\delta_{\mathrm{m}}}\right) r_{\mathrm{o}}}+B e^{-\left(\frac{1-i}{\delta_{\mathrm{m}}}\right) r_{\mathrm{o}}}, \\
& \bar{X}=-B e^{-\left(\frac{1-i}{\delta_{\mathrm{m}}}\right)\left(r_{\mathrm{o}}+2 \Delta\right)}+B e^{-\left(\frac{1-i}{\delta_{\mathrm{m}}}\right) r_{\mathrm{o}}}, \\
& \bar{X}=B e^{-\left(\frac{1-i}{\delta_{\mathrm{m}}}\right) r_{\mathrm{o}}}\left(1-e^{-\left(\frac{1-i}{\delta_{\mathrm{m}}}\right)(2 \Delta)}\right) \\
& B=\bar{X} \frac{e^{\left(\frac{1-i}{\delta_{\mathrm{m}}}\right) r_{\mathrm{o}}}}{\left(1-e^{-\left(\frac{1-i}{\delta_{\mathrm{m}}}\right)(2 \Delta)}\right)}
\end{aligned}
$$

and

$$
A=-\bar{X} \frac{e^{-\left(\frac{1-i}{\delta_{\mathrm{m}}}\right)\left(r_{\mathrm{o}}+2 \Delta\right)}}{\left(1-e^{-\left(\frac{1-i}{\delta_{\mathrm{m}}}\right)(2 \Delta)}\right)}
$$


Eventually, equation (A.48) becomes, for $X=r \widetilde{B}_{\mathrm{t}_{\ell}}^{m}$ and $\bar{X}=r_{\mathrm{o}} \widetilde{B}_{\mathrm{t}_{\ell}}^{m}\left(r_{\mathrm{o}}\right)$,

$$
\begin{aligned}
& X=-\bar{X} \frac{e^{-\left(\frac{1-i}{\delta_{\mathrm{m}}}\right)\left(r_{\mathrm{o}}+2 \Delta\right)}}{\left(1-e^{-\left(\frac{1-i}{\delta_{\mathrm{m}}}\right)(2 \Delta)}\right)} e^{\left(\frac{1-i}{\delta_{\mathrm{m}}}\right) r}+\bar{X} \frac{e^{\left(\frac{1-i}{\delta_{\mathrm{m}}}\right) r_{\mathrm{o}}}}{\left(1-e^{-\left(\frac{1-i}{\delta_{\mathrm{m}}}\right)(2 \Delta)}\right)} e^{-\left(\frac{1-i}{\delta_{\mathrm{m}}}\right) r} \\
& X=-\bar{X} \frac{e^{-\left(\frac{1-i}{\delta_{\mathrm{m}}}\right)\left(r_{\mathrm{o}}+2 \Delta-r\right)}}{\left(1-e^{-\left(\frac{1-i}{\delta_{\mathrm{m}}}\right)(2 \Delta)}\right)}+\bar{X} \frac{e^{\left(\frac{1-i}{\delta_{\mathrm{m}}}\right)\left(r_{\mathrm{o}}-r\right)}}{\left(1-e^{-\left(\frac{1-i}{\delta_{\mathrm{m}}}\right)(2 \Delta)}\right)} \\
& \bar{X}=\frac{e^{\left(\frac{1-i}{\delta_{\mathrm{m}}}\right)\left(r_{\mathrm{o}}-r\right)}-e^{-\left(\frac{1-i}{\delta_{\mathrm{m}}}\right)\left(r_{\mathrm{o}}+2 \Delta-r\right)}}{1-e^{-\left(\frac{1-i}{\delta_{\mathrm{m}}}\right)(2 \Delta)}}
\end{aligned}
$$

The solution (A.61) can be rewritten using the hyperbolic sine function defined as

$$
\sinh (x)=\frac{e^{x}-e^{-x}}{2}
$$

leading to

$$
\frac{X}{\bar{X}}\left(r>r_{\mathrm{o}}, \omega\right)=\frac{\sinh \left[\left(\frac{1-i}{\delta_{\mathrm{m}}}\right)\left(r_{\mathrm{o}}+\Delta-r\right)\right]}{\sinh \left[\left(\frac{1-i}{\delta_{\mathrm{m}}}\right) \Delta\right]}
$$

\section{Interface conditions}

\section{Mantle-side}

Taking the latitudinal derivative of this expression gives

$$
\left.\frac{\partial B_{\mathrm{t}}}{\partial \theta}\right|_{\text {mantle }}=r_{\mathrm{o}} \widetilde{B}_{\mathrm{t}_{\ell}}^{m}\left(r_{\mathrm{o}}\right) \frac{\sinh \left[\left(\frac{1-i}{\delta_{\mathrm{m}}}\right)\left(r_{\mathrm{o}}+\Delta-r\right)\right]}{\sinh \left[\left(\frac{1-i}{\delta_{\mathrm{m}}}\right) \Delta\right]} \times \frac{\partial}{\partial \theta}\left(\sum_{\ell, m}^{\mathrm{L}, \mathrm{M}} Y_{\ell}^{m}(\theta, \varphi)\right),
$$

so that

$$
\begin{aligned}
\left.\frac{\partial}{\partial r} \frac{\partial B_{\mathrm{t}}}{\partial \theta}\right|_{\text {mantle }} & =r_{\mathrm{o}} \widetilde{B}_{\mathrm{t}_{\ell}}^{m}\left(r_{\mathrm{o}}\right) \frac{\partial}{\partial r}\left(\frac{\sinh \left[\left(\frac{1-i}{\delta_{\mathrm{m}}}\right)\left(r_{\mathrm{o}}+\Delta-r\right)\right]}{\sinh \left[\left(\frac{1-i}{\delta_{\mathrm{m}}}\right) \Delta\right]}\right) \\
& \times \frac{\partial}{\partial \theta}\left(\sum_{\ell, m}^{\mathrm{L}, \mathrm{M}} Y_{\ell}^{m}(\theta, \varphi)\right)
\end{aligned}
$$


and as $\sinh (x)^{\prime}=\cosh (x)$, we have

$$
\begin{aligned}
\left.\frac{\partial}{\partial r} \frac{\partial B_{\mathrm{t}}}{\partial \theta}\right|_{\text {mantle }} & =-\left(\frac{1-i}{\delta_{\mathrm{m}}}\right) r_{\mathrm{o}} \widetilde{B}_{\mathrm{t}_{\ell}}^{m}\left(r_{\mathrm{o}}\right) \frac{\cosh \left[\left(\frac{1-i}{\delta_{\mathrm{m}}}\right)\left(r_{\mathrm{o}}+\Delta-r\right)\right]}{\sinh \left[\left(\frac{1-i}{\delta_{\mathrm{m}}}\right) \Delta\right]} \\
& \times \frac{\partial}{\partial \theta}\left(\sum_{\ell, m}^{\mathrm{L}, \mathrm{M}} Y_{\ell}^{m}(\theta, \varphi)\right) .
\end{aligned}
$$

The evaluation of this expression at the mantle side of the CMB, in $r=r_{\mathrm{o}}$, gives

$$
\left.\frac{\partial}{\partial r} \frac{\partial B_{\mathrm{t}}}{\partial \theta}\right|_{\text {mantle }} ^{r_{\mathrm{o}}}=-\left(\frac{1-i}{\delta_{\mathrm{m}}}\right) r_{\mathrm{o}} \widetilde{B}_{\mathrm{t}_{\ell}}^{m}\left(r_{\mathrm{o}}\right) \frac{\cosh \left[\left(\frac{1-i}{\delta_{\mathrm{m}}}\right) \Delta\right]}{\sinh \left[\left(\frac{1-i}{\delta_{\mathrm{m}}}\right) \Delta\right]} \times \frac{\partial}{\partial \theta}\left(\sum_{\ell, m}^{\mathrm{L}, \mathrm{M}} Y_{\ell}^{m}(\theta, \varphi)\right)
$$

in which appear a hyperbolic cotangent as

$$
\operatorname{coth}(x)=\frac{\cosh (x)}{\sinh (x)}
$$




\section{A.3 Elementary Cases: Validation of the Electromag- netic torque models}

\section{Solid-body rotation}

The reduced system of the dimensionless conservation of the angular momentum of the inner core and the mantle is

$$
\begin{aligned}
& \Omega_{\mathrm{ic}}\left(\Psi_{1}+\Psi_{\mathrm{ic}}-i \omega\right)=\Psi_{1} \Omega_{\mathrm{oc}}+\Psi_{\mathrm{ic}} \Omega_{\mathrm{m}} \\
& \Omega_{\mathrm{m}}\left(\Psi_{2}+\Psi_{\mathrm{m}}-i \omega\right)=\Psi_{2} \Omega_{\mathrm{oc}}+\Psi_{\mathrm{m}} \Omega_{\mathrm{ic}} .
\end{aligned}
$$

with

$$
\begin{array}{ll}
\Psi_{1}=\frac{K_{1}\left(\frac{1+i}{4}\right) \sqrt{\frac{2}{\omega}} r_{\mathrm{i}}^{4} B_{r_{\mathrm{i}}}^{2} \mathcal{L} u^{2}}{\mathrm{I}_{\mathrm{i}}}, & \Psi_{\mathrm{ic}}=\frac{\zeta_{\eta}}{\mathrm{I}_{\mathrm{i}}}, \\
\Psi_{2}=\frac{K_{2} r_{\mathrm{o}}^{4} B_{r_{\mathrm{o}}}^{2} \Sigma \mathcal{L} u^{2}}{\mathrm{I}_{\mathrm{m}}}, & \Psi_{\mathrm{ic}}=\frac{\zeta_{\eta}}{\mathrm{I}_{\mathrm{m}}} .
\end{array}
$$

This can be further simplified by introducing

$$
\gamma_{c}=\Psi_{1}+\Psi_{\mathrm{ic}}-i \omega, \quad \text { and } \quad \gamma_{m}=\Psi_{2}+\Psi_{\mathrm{m}}-i \omega
$$

leading to

$$
\begin{aligned}
\Omega_{\mathrm{ic}} \gamma_{c} & =\Psi_{1} \Omega_{\mathrm{oc}}+\Psi_{\mathrm{ic}} \Omega_{\mathrm{m}}, \\
\Omega_{\mathrm{m}} \gamma_{m} & =\Psi_{2} \Omega_{\mathrm{oc}}+\Psi_{\mathrm{m}} \Omega_{\mathrm{ic}} .
\end{aligned}
$$

The angular velocity of the inner core may then be obtained like

$$
\begin{aligned}
\Omega_{\mathrm{ic}} \gamma_{c} & =\Psi_{1} \Omega_{\mathrm{oc}}+\frac{\Psi_{\mathrm{ic}}}{\gamma_{m}}\left(\Psi_{2} \Omega_{\mathrm{oc}}-\Psi_{\mathrm{m}} \Omega_{\mathrm{ic}}\right) \\
\Omega_{\mathrm{ic}}\left(\gamma_{c} \gamma_{m}-\Psi_{\mathrm{ic}} \Psi_{\mathrm{m}}\right) & =\left(\Psi_{1} \gamma_{m}+\Psi_{\mathrm{ic}} \Psi_{2}\right) \Omega_{\mathrm{oc}} \\
\Omega_{\mathrm{ic}} & =\Omega_{\mathrm{oc}}\left(\frac{\Psi_{1} \gamma_{m}+\Psi_{\mathrm{ic}} \Psi_{2}}{\gamma_{c} \gamma_{m}-\Psi_{\mathrm{ic}} \Psi_{\mathrm{m}}}\right)
\end{aligned}
$$


And, introducing the last expression of $\Omega_{\text {ic }}$ into equation (A.72) gives

$$
\begin{aligned}
\Omega_{\mathrm{m}} \gamma_{m} & =\Psi_{2} \Omega_{\mathrm{oc}}+\Psi_{\mathrm{m}} \Omega_{\mathrm{ic}} \\
\Omega_{\mathrm{m}} \gamma_{m} & =\Psi_{2} \Omega_{\mathrm{oc}}+\Psi_{\mathrm{m}} \Omega_{\mathrm{oc}}\left(\frac{\Psi_{1} \gamma_{m}+\Psi_{\mathrm{ic}} \Psi_{2}}{\gamma_{c} \gamma_{m}-\Psi_{\mathrm{ic}} \Psi_{\mathrm{m}}}\right), \\
\Omega_{\mathrm{m}} & =\frac{\Omega_{\mathrm{oc}}}{\gamma_{m}}\left(\Psi_{2}+\Psi_{\mathrm{m}} \times \frac{\Psi_{1} \gamma_{m}+\Psi_{\mathrm{ic}} \Psi_{2}}{\gamma_{c} \gamma_{m}-\Psi_{\mathrm{ic}} \Psi_{\mathrm{m}}}\right), \\
\Omega_{\mathrm{m}} & =\frac{\Omega_{\mathrm{oc}}}{\gamma_{m}}\left(\frac{\Psi_{2} \gamma_{c} \gamma_{m}-\Psi_{2} \Psi_{\mathrm{ic}} \Psi_{\mathrm{m}}+\Psi_{\mathrm{m}} \Psi_{1} \gamma_{m}+\Psi_{\mathrm{m}} \Psi_{\mathrm{ic}} \Psi_{2}}{\gamma_{c} \gamma_{m}-\Psi_{\mathrm{ic}} \Psi_{\mathrm{m}}}\right), \\
\Omega_{\mathrm{m}} & =\Omega_{\mathrm{oc}}\left(\frac{\Psi_{2} \gamma_{c}+\Psi_{\mathrm{m}} \Psi_{1}}{\gamma_{c} \gamma_{m}-\Psi_{\mathrm{ic}} \Psi_{\mathrm{m}}}\right) .
\end{aligned}
$$

With the expression of $\Omega_{\mathrm{ic}}$ and $\Omega_{\mathrm{m}}$, we can now describe the inner core super-rotation

$$
\begin{aligned}
\mathcal{S}=\Omega_{\mathrm{ic}}-\Omega_{\mathrm{m}} & =\Omega_{\mathrm{oc}}\left(\frac{\Psi_{1} \gamma_{m}+\Psi_{\mathrm{ic}} \Psi_{2}}{\gamma_{c} \gamma_{m}-\Psi_{\mathrm{ic}} \Psi_{\mathrm{m}}}\right)-\Omega_{\mathrm{oc}}\left(\frac{\Psi_{2} \gamma_{c}+\Psi_{\mathrm{m}} \Psi_{1}}{\gamma_{c} \gamma_{m}-\Psi_{\mathrm{ic}} \Psi_{\mathrm{m}}}\right), \\
\mathcal{S} & =\Omega_{\mathrm{oc}}\left(\frac{\Psi_{1} \gamma_{m}+\Psi_{\mathrm{ic}} \Psi_{2}-\Psi_{2} \gamma_{c}-\Psi_{\mathrm{m}} \Psi_{1}}{\gamma_{c} \gamma_{m}-\Psi_{\mathrm{ic}} \Psi_{\mathrm{m}}}\right)
\end{aligned}
$$

and the geomagnetic westward drift,

$$
\begin{aligned}
& \mathcal{D}=\Omega_{\mathrm{oc}}-\Omega_{\mathrm{m}}=\Omega_{\mathrm{oc}}-\Omega_{\mathrm{oc}}\left(\frac{\Psi_{2} \gamma_{c}+\Psi_{\mathrm{m}} \Psi_{1}}{\gamma_{c} \gamma_{m}-\Psi_{\mathrm{ic}} \Psi_{\mathrm{m}}}\right), \\
& \mathcal{D}=\Omega_{\mathrm{oc}}\left(1-\frac{\Psi_{2} \gamma_{c}+\Psi_{\mathrm{m}} \Psi_{1}}{\gamma_{c} \gamma_{m}-\Psi_{\mathrm{ic}} \Psi_{\mathrm{m}}}\right)
\end{aligned}
$$

when we impose a periodic solid-body rotation in the outer core, with angular frequency $\omega$.

\section{Shear in the fluid outer core}

In the case of an imposed periodic shear flow in fluid outer core that bears no angular momentum, the angular momentum conservation of the inner core, the mantle and the fluid outer is described by

$$
\begin{aligned}
& -i \omega \Omega_{\mathrm{ic}}=\Psi_{1}\left(\Omega_{\mathrm{ficb}}-\Omega_{\mathrm{ic}}\right)-\Psi_{\mathrm{ic}}\left(\Omega_{\mathrm{ic}}-\Omega_{\mathrm{m}}\right), \\
& -i \omega \Omega_{\mathrm{m}}=\Psi_{2}\left(\Omega_{\mathrm{fcmb}}-\Omega_{\mathrm{m}}\right)+\Psi_{\mathrm{m}}\left(\Omega_{\mathrm{ic}}-\Omega_{\mathrm{m}}\right) \text {, } \\
& 0=-\Psi_{1} \mathrm{I}_{\mathrm{i}}\left(\Omega_{\mathrm{ficb}}-\Omega_{\mathrm{ic}}\right)-\Psi_{2} \mathrm{I}_{\mathrm{m}}\left(\Omega_{\mathrm{fcmb}}-\Omega_{\mathrm{m}}\right) \text {. }
\end{aligned}
$$

This can be transformed by using the expressions of the inner core super rotation $\mathcal{S}=$ $\Omega_{\mathrm{ic}}-\Omega_{\mathrm{m}}$, the geomagnetic westward drift $\mathcal{D}=\Omega_{\mathrm{fcmb}}-\Omega_{\mathrm{m}}$ and the global shear in the fluid 
outer core $\mathcal{C}_{\mathrm{f}}=\Omega_{\text {ficb }}-\Omega_{\mathrm{fcmb}}$,

$$
\begin{aligned}
-i \omega \Omega_{\mathrm{ic}} & =\Psi_{1}\left(\mathcal{C}_{\mathrm{f}}+\mathcal{D}-\mathcal{S}\right)-\Psi_{\mathrm{ic}} \mathcal{S} \\
-i \omega \Omega_{\mathrm{m}} & =\Psi_{2} \mathcal{D}+\Psi_{\mathrm{m}} \mathcal{S} \\
0 & =-\Psi_{1} \mathrm{I}_{\mathrm{i}}\left(\mathcal{C}_{\mathrm{f}}+\mathcal{D}-\mathcal{S}\right)-\Psi_{2} \mathrm{I}_{\mathrm{m}} \mathcal{D} .
\end{aligned}
$$

The operation (A.87)-(A.88) leads to

$$
\begin{aligned}
-i \omega \mathcal{S} & =\Psi_{1}\left(\mathcal{C}_{\mathrm{f}}+\mathcal{D}-\mathcal{S}\right)-\Psi_{\mathrm{ic}} \mathcal{S}-\Psi_{2} \mathcal{D}-\Psi_{\mathrm{m}} \mathcal{S} \\
\mathcal{S}\left(\Psi_{1}+\Psi_{\mathrm{ic}}+\Psi_{\mathrm{m}}-i \omega\right) & =\Psi_{1} \mathcal{C}_{\mathrm{f}}+\mathcal{D}\left(\Psi_{1}-\Psi_{2}\right)
\end{aligned}
$$

while equation (A.89) is equivalent to

$$
\mathcal{D}=-\gamma_{\mathrm{f}}\left(\mathcal{C}_{\mathrm{f}}-\mathcal{S}\right)
$$

with

$$
\gamma_{\mathrm{f}}=\frac{\Psi_{1} \mathrm{I}_{\mathrm{i}}}{\Psi_{1} \mathrm{I}_{\mathrm{i}}+\Psi_{2} \mathrm{I}_{\mathrm{m}}} .
$$

By introducing the expression of $\mathcal{D}$ (Eq.A.92) into equation (A.91) we obtain

$$
\mathcal{S}\left(\Psi_{1}+\Psi_{\mathrm{ic}}+\Psi_{\mathrm{m}}-i \omega\right)=\Psi_{1} \mathcal{C}_{\mathrm{f}}-\gamma_{\mathrm{f}}\left(\mathcal{C}_{\mathrm{f}}-\mathcal{S}\right)\left(\Psi_{1}-\Psi_{2}\right)
$$

and,

$$
\mathcal{S}\left(\Psi_{1}+\Psi_{\mathrm{ic}}+\Psi_{\mathrm{m}}-\gamma_{\mathrm{f}}\left(\Psi_{1}-\Psi_{2}\right)-i \omega\right)=\mathcal{C}_{\mathrm{f}}\left[\Psi_{1}-\gamma_{\mathrm{f}}\left(\Psi_{1}-\Psi_{2}\right)\right]
$$

and ultimately,

$$
\mathcal{S}=\mathcal{C}_{\mathrm{f}} \frac{\Psi_{1}-\gamma_{\mathrm{f}}\left(\Psi_{1}-\Psi_{2}\right)}{\Psi_{1}+\Psi_{\mathrm{ic}}+\Psi_{\mathrm{m}}-\gamma_{\mathrm{f}}\left(\Psi_{1}-\Psi_{2}\right)-i \omega}
$$

This expression then leads to the formulation of the geomagnetic westward drift like

$$
\mathcal{D}=-\mathcal{C}_{\mathrm{f}} \gamma_{\mathrm{f}}\left(1-\frac{\Psi_{1}-\gamma_{\mathrm{f}}\left(\Psi_{1}-\Psi_{2}\right)}{\Psi_{1}+\Psi_{\mathrm{ic}}+\Psi_{\mathrm{m}}-\gamma_{\mathrm{f}}\left(\Psi_{1}-\Psi_{2}\right)-i \omega}\right)
$$




\section{List of Figures}

$\begin{array}{ll}\text { Introduction } & 7\end{array}$

1.1 Les premières boussoles . . . . . . . . . . . . . . . . . . . . . . . . . 9

1.2 W.Gilbert, De Magnete. . . . . . . . . . . . . . . . . . . . . 11

1.3 E. Halley, la dérive vers l'ouest . . . . . . . . . . . . . . . . . . 12

1.4 L'intérieur de la Terre . . . . . . . . . . . . . . . . . . . . . . . . . 13

1.5 Simulations numériques (Glatzmaier and Roberts, 1995 \& Aubert et al., 2013) 14

1.6 Preliminary Reference Earth Model (Dziewonski and Anderson, 1981) . . . 17

1.7 Magnetic field vector . . . . . . . . . . . . . . . . . 19

1.8 Map of declination measurements (Jackson et al., 2003) . . . . . . . . . . 20

1.9 External sources of magnetic field (Grayver et al., 2016) . . . . . . . . . . 21

1.10 The magnetic curtain (Roberts and King, 2013) . . . . . . . . . . . . . 23

1.11 Spherical harmonic representation . . . . . . . . . . . . . . . . . 24

1.12 Maps of Earth's magnetic field (2017) . . . . . . . . . . . . . . . . 26

1.13 Maps of the secular variation $(2017) \ldots \ldots \ldots$. . . . . . . . . . 27

1.14 The geomagnetic westward drift (Finlay, $2003-1 / 2) \ldots \ldots . . \ldots 28$

1.15 The geomagnetic westward drift (Finlay, $2003-2 / 2) \ldots . . . .29$

1.16 Braking mechanism of the inner core super-rotation (Mound and Buffett, 2003) 32

1.17 Phase diagram of iron $($ Tateno, 2010) . . . . . . . . . . . . . . 33

1.18 Inner core super-rotation estimates in the literature . . . . . . . . . . . 35

1.19 Coupled-Earth model (Aubert et al., 2013) . . . . . . . . . . . . . . 37

1.20 Rotational dynamics of the study . . . . . . . . . . . . . . . . 41

$\begin{array}{ll}\text { Numerical implementation } & 59\end{array}$

3.1 Radial discretization ... . . . . . . . . . . . . . . . 64

3.2 Thin layer approximation . . . . . . . . . . . . . . . . 71

3.3 Leakage versus advective torque . . . . . . . . . . . . . . . . 73

$\begin{array}{ll}\text { Long-term rotational dynamics } & 75\end{array}$

4.1 Scheme of the rotational dynamics . . . . . . . . . . . . 88 
4.2 Meridional sections of $V_{\varphi}$ and $B_{\varphi} \ldots \ldots \ldots \ldots \ldots$

4.3 Shears as functions of the state of coupling . . . . . . . . . . . . . . 94

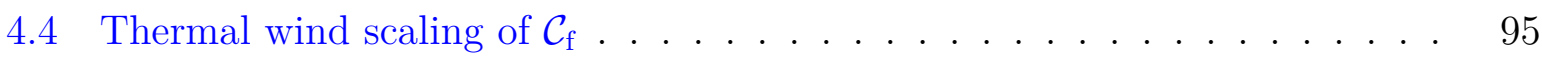

4.5 Electromagnetic torque at the $\mathrm{CMB}-1 / 2 \ldots \ldots$. . . . . . . . 95

4.6 Electromagnetic torque at the $\mathrm{CMB}-2 / 2 \ldots \ldots$. . . . . . . . . . 96

4.7 Local versus remote contributions . . . . . . . . . . . . . . . . . . 97

4.8 Electromagnetic torque at the ICB f . . . . . . . . . . . . . . . . 98

4.9 Diagrams of $\mathcal{S}$ and $\mathcal{D} \ldots \ldots \ldots \ldots \ldots$

4.10 Errors on torque balance . . . . . . . . . . . . . . . . . . . 102

4.11 Statistical convergence . . . . . . . . . . . . . . . . . . 103

Time-dependent electromagnetic torques 103

5.1 Scheme of the perturbed state $-1 / 4 \ldots \ldots \ldots$. . . . . . . . 107

5.2 Scheme of the perturbed state $-2 / 4 \ldots \ldots \ldots \ldots$. . . . . . . . 111

5.3 Thin layer approximation . . . . . . . . . . . . . . . . . 112

5.4 Thin versus thick electrically conducting layer . . . . . . . . . . . . . . . . 114

5.5 Scheme of the perturbed state $-3 / 4 \ldots \ldots \ldots \ldots$

5.6 Scheme of the perturbed state $-4 / 4 \ldots \ldots \ldots$. . . . . . . . . 117

5.7 Phase lag of $B_{\varphi}$ and conclusions . . . . . . . . . . . . . . 125

Elementary cases: validation of the electromagnetic torque models $\quad 127$

6.1 Solid-body rotation . . . . . . . . . . . . . . . . . . . 132

6.2 Transient to stationary regime . . . . . . . . . . . . . . . . . 133

6.3 Shear at ICB and $\widetilde{\Gamma}_{\mathrm{ICB}}^{1} \ldots \ldots \ldots \ldots \ldots \ldots \ldots$

6.4 Scaling of $\widetilde{\Gamma}_{\mathrm{ICB}}^{1} \ldots \ldots \ldots \ldots \ldots \ldots \ldots$

6.5 Shear at the ICB with gravitational coupling . . . . . . . . . . . 136

6.6 Amplitude of $\widetilde{\Gamma}_{\text {ICB }}^{1}$ with gravitational coupling . . . . . . . . . . . . . . . . . . . . . . . . . . . . 138

6.7 Scaling of $\widetilde{\Gamma}_{\mathrm{ICB}}^{1}$ with gravitational coupling . . . . . . . . . . . . . . . . . . . . . . . . . . . . . . . . . . .

6.8 Solid-body case: observed and modeled $\mathcal{S}$. . . . . . . . . . . . . . . . . . . 141

6.9 Solid-body case: observed and modeled $\mathcal{S}$ with gravitational coupling . . . 142

6.10 Shear flow in the fluid outer core . . . . . . . . . . . . . . . . . . 143

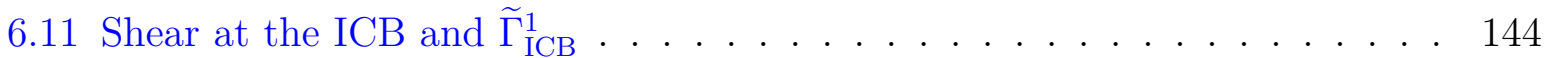

6.12 Phase lag between the shear at the ICB and $\widetilde{\Gamma}_{\mathrm{ICB}}^{1} \ldots \ldots \ldots \ldots$

6.13 Snapshots of $B_{\varphi}$ - Solid-body versus shear flow . . . . . . . . . . . 146

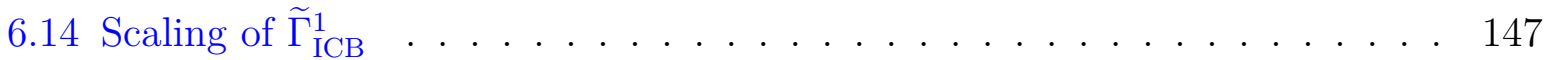

6.15 Shear flow case: observed and modeled $\mathcal{S} \ldots \ldots \ldots$. . . . . . . . . . . . . . 148

Amplitude of rotational fluctuations in geodynamo simulations $\quad 149$

7.1 Shears as functions of the strength of gravitational coupling . . . . . . . 153 
7.2 Shears as functions of the lower mantle conductance . . . . . . . . . . . . . 154

7.3 Electromagnetic torque at the ICB . . . . . . . . . . . . . . . . 155

7.4 Electromagnetic torque at the CMB . . . . . . . . . . . . . . . . 155

7.5 Geodynamo case: observed and modeled $\mathcal{S} \ldots \ldots \ldots$. . . . . . . 156

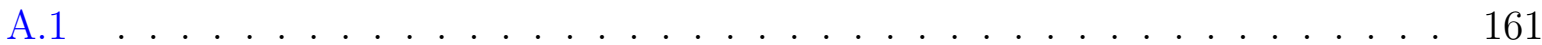




\section{Bibliography}

Alexandrescu, M., D. Gibert, J. Le Mouël, G. Hulot, and G. Saracco (1999). An estimate of average lower mantle conductivity by wavelet analysis of geomagnetic jerks. Journal of Geophysical Research: Solid Earth 104(B8), 17735-17745. doi: 10.1029/1999JB900135.

Allègre, C., J. Poirier, E. Humler, and A. Hofmann (1995). The chemical composition of the Earth. Earth and Planetary Science Letters 134(3-4), 515-526. doi: 10.1016/0012821X(95)00123-T.

Anufriev, A., C. Jones, and A. Soward (2005). The Boussinesq and anelastic liquid approximations for convection in the Earth's core. Physics of the Earth and Planetary Interiors 152(3), 163-190. doi: 10.1016/j.pepi.2005.06.004.

Aubert, J. (2005). Steady zonal flows in spherical shell dynamos. Journal of Fluid Mechanics 542, 53-67. doi: 10.1017/S0022112005006129.

Aubert, J. (2013). Flow throughout the Earth's core inverted from geomagnetic observations and numerical dynamo models. Geophysical Journal International 192(2), 537-556. doi: $10.1093 /$ gji/ggs051.

Aubert, J., H. Amit, G. Hulot, and P. Olson (2008). Thermochemical flows couple the Earth's inner core growth to mantle heterogeneity. Nature 454(7205), 758-761. doi: 10.1038/nature07109.

Aubert, J., J. Aurnou, and J. Wicht (2008). The magnetic structure of convection-driven numerical dynamos. Geophysical Journal International 172, 945-956. doi: 10.1111/j.1365246X.2007.03693.x.

Aubert, J. and M. Dumberry (2011). Steady and fluctuating inner core rotation in numerical geodynamo models. Geophysical Journal International 184(1), 162-170. doi: 10.1111/j.1365-246X.2010.04842.x.

Aubert, J., C. Finlay, and A. Fournier (2013). Bottom-up control of geomagnetic secular variation by the Earth's inner core. Nature 502(7470), 219+. doi: 10.1038/nature12574. 
Aubert, J., T. Gastine, and A. Fournier (2017). Spherical convective dynamos in the rapidly rotating asymptotic regime. Journal of Fluid Mechanics 813, 558-593. doi: 10.1017/jfm.2016.789.

Aubert, J., S. Labrosse, and C. Poitou (2009). Modelling the palaeo-evolution of the geodynamo. Geophysical Journal International 179(3), 1414-1428. doi: 10.1111/j.1365246X.2009.04361.x.

Aurnou, J., S. Andreadis, L. Zhu, and P. Olson (2003). Experiments on convection in Earth's core tangent cylinder. Earth and Planetary Science Letters 212(1), 119-134. doi: 10.1016/S0012-821X(03)00237-1.

Aurnou, J., D. Brito, and P. Olson (1996). Mechanics of inner core super-rotation. Geophysical Research Letters 23(23), 3401-3404. doi: 10.1029/96GL03258.

Aurnou, J., D. Brito, and P. Olson (1998). Anomalous rotation of the inner core and the toroidal magnetic field. Journal of Geophysical Research 103(B5), 9721-9738. doi: 10.1029/97JB03618.

Backus, G. (1986). Poloidal and toroidal fields in geomagnetic field modeling. Reviews of Geophysics 24(1), 75-109. doi: 10.1029/RG024i001p00075.

Backus, G. E. (1968). Kinematics of geomagnetic secular variation in a perfectly conducting core. Phil. Trans. R. Soc. Lond. A 263(1141), 239-266.

Bauer, L. (1895). On the secular motion of a free magnetic needle. Physical Review (Series I) 2(6), 455. doi: 10.1126/science.ns-20.506.218.

Belonoshko, A., R. Ahuja, and B. Johansson (2003). Stability of the body-centred-cubic phase of iron in the Earth's inner core. Nature 424(6952), 1032. doi: 10.1038/nature01954.

Bergman, M., S. Agrawal, M. Carter, and M. Macleod-Silberstein (2003). Transverse solidification textures in hexagonal close-packed alloys. Journal of crystal growth 255(1), 204-211. doi: 10.1016/S0022-0248(03)01247-8.

Bergman, M., M. Macleod-Silberstein, M. Haskel, B. Chandler, and N. Akpan (2005). A laboratory model for solidification of Earth's core. Physics of the Earth and Planetary Interiors 153(1), 150-164. doi: 10.1016/j.pepi.2005.03.016.

Birch, F. (1952). Elasticity and constitution of the Earth's interior. Journal of Geophysical Research 57(2), 227-286. doi: 10.1029/JZ057i002p00227.

Bloxham, J. (1987). Simultaneous stochastic inversion for geomagnetic main field and secular variation: 1. A large-scale inverse problem. Journal of Geophysical Research: Solid Earth 92(B11), 11597-11608. doi: 10.1029/JB092iB11p11597. 
Bloxham, J., D. Gubbins, and A. Jackson (1989). Geomagnetic secular variation. Philosophical Transactions of the Royal Society of London A: Mathematical, Physical and Engineering Sciences 329(1606), 415-502. doi: 10.1098/rsta.1989.0087.

Bloxham, J. and A. Jackson (1991). Fluid flow near the surface of Earth's outer core. Reviews of Geophysics 29(1), 97-120. doi: 10.1029/90RG02470.

Bloxham, J. and A. Jackson (1992). Time-dependent mapping of the magnetic field at the core-mantle boundary. Journal of Geophysical Research: Solid Earth 97(B13), 19537-19563. doi: 10.1029/92JB01591.

Bloxham, J., S. Zatman, and M. Dumberry (2002). The origin of geomagnetic jerks. Nature 420(6911), 65. doi: 10.1038/nature01134.

Braginsky, S. (1963). Structure of the F layer and reasons for convection in the Earth's core. In Doklady Akad. Nauk SSSR, Volume 149, pp. 8-10.

Braginsky, S. (1970). Torsional magnetohydrodynamic vibrations in the Earth's core and variations in day length. Geomagn. Aeron..

Braginsky, S. and P. Roberts (1995). Equations governing convection in earth's core and the geodynamo. Geophysical \& Astrophysical Fluid Dynamics 79(1-4), 1-97. doi: $10.1080 / 03091929508228992$.

Buffett, B. (1992). Constraints on magnetic energy and mantle conductivity from the forced nutations of the earth. Journal of Geophysical Research 97(B13), 19581-19597. doi: 10.1029/92JB00977.

Buffett, B. (1996a). A mechanism for decade fluctuations in the length of day. Geophysical Research Letters 23(25), 3803-3806. doi: 10.1029/96GL03571.

Buffett, B. (1996b). Gravitational oscillations in the length of day. Geophysical Research Letters 23(17), 2279-2282. doi: 10.1029/96GL02083.

Buffett, B. (1997). Geodynamic estimates of the viscosity of the Earth's inner core. Nature 388(6642), 571-573. doi: 10.1038/41534.

Buffett, B. (1998). Free oscillations in the length of day: inferences on physical properties near the core-mantle boundary. The core-mantle boundary region, 153-165. doi: 10.1029/GD028p0153.

Buffett, B. and U. Christensen (2007). Magnetic and viscous coupling at the core-mantle boundary: inferences from observations of the Earth's nutations. Geophysical Journal International 171(1), 145-152. doi: 10.1111/j.1365-246X.2007.03543.x. 
Buffett, B. and G. Glatzmaier (2000). Gravitational braking of inner-core rotation in geodynamo simulations. Geophysical Research Letters 27(19), 3125-3128. doi: 10.1029/2000GL011705.

Buffett, B., P. Mathews, and T. Herring (2002). Modeling of nutation and precession: Effects of electromagnetic coupling. Journal of Geophysical Research 107(B4). doi: 10.1029/2000JB000056.

Bullard, E., C. Freedman, H. Gellman, and J. Nixon (1950). The westward drift of the Earth's magnetic field. Philosophical Transactions of the Royal Society of London. Series A. Mathematical and Physical Sciences, 67-92. doi: 10.1098/rsta.1950.0014.

Bullard, E. and H. Gellman (1954). Homogeneous dynamos and terrestrial magnetism. Philosophical Transactions of the Royal Society of London. Series A, Mathematical and Physical Sciences, 213-278. doi: 10.1098/rsta.1954.0018.

Busse, F. H. (1970). Thermal instabilities in rapidly rotating systems. Journal of Fluid Mechanics 44(3), 441-460. doi: 10.1017/S0022112070001921.

Cain, J., W. Daniels, S. Hendricks, and D. Jensen (1965). An evaluation of the main geomagnetic field, 1940-1962. Journal of Geophysical Research 70 (15), 3647-3674. doi: 10.1029/JZ070i015p03647.

Cain, J., S. Hendricks, R. Langel, and W. Hudson (1967). A proposed model for the International Geomagnetic Reference Field-1965. Journal of Geomagnetism and Geoelectricity 19(4), 335-355. doi: 10.5636/jgg.19.335.

Carrigan, C. and F. Busse (1983). An experimental and theoretical investigation of the onset of convection in rotating spherical shells. Journal of Fluid Mechanics 126, 287-305. doi: $10.1017 /$ S0022112083000166.

Christensen, U. and J. Aubert (2006). Scaling properties of convection-driven dynamos in rotating spherical shells and application to planetary magnetic fields. Geophysical Journal International 166(1), 97-114. doi: 10.1111/j.1365-246X.2006.03009.x.

Christensen, U., J. Aubert, P. Cardin, E. Dormy, S. Gibbons, G. Glatzmaier, E. Grote, Y. Honkura, C. Jones, M. Kono, M. Matsushima, A. Sakuraba, F. Takahashi, A. Tilgner, J. Wicht, and K. Zhang (2001). A numerical dynamo benchmark. Physics of the Earth and Planetary Interiors 128(1-4), 25-34. doi: 10.1016/S0031-9201(01)00275-8.

Christensen, U., J. Aubert, and G. Hulot (2010). Conditions for Earth-like geodynamo models. Earth and Planetary Science Letters 296(3-4), 487-496. doi: 10.1016/j.epsl.2010.06.009. 
Christensen, U. and P. Olson (2003). Secular variation in numerical geodynamo models with lateral variations of boundary heat flow. Physics of the Earth and Planetary Interiors 138(1), 39-54. doi: 10.1016/S0031-9201(03)00064-5.

Civet, F., E. Thébault, O. Verhoeven, B. Langlais, and D. Saturnino (2015). Electrical conductivity of the Earth's mantle from the first Swarm magnetic field measurements. Geophysical Research Letters 42(9), 3338-3346. doi: 10.1002/2015GL063397.

Creager, K. (1992). Anisotropy of the inner core from differential travel times of the phases PKP and PKIKP. Nature 356(6367), 309. doi: 10.1038/356309a0.

Creager, K. (1997). Inner core rotation rate from small-scale heterogeneity and time-varying travel times. Science 278(5341), 1284-1288. doi: 10.1126/science.278.5341.1284.

Davies, C., D. Stegman, and M. Dumberry (2014). The strength of gravitational core-mantle coupling. Geophysical Research Letters 41. doi: 10.1002/2014GL059836.

de Koker, N., G. Steinle-Neumann, and V. Vlček (2012). Electrical resistivity and thermal conductivity of liquid Fe alloys at high $\mathrm{P}$ and T, and heat flux in Earth's core. Proceedings of the National Academy of Sciences 109(11), 4070-4073. doi: 10.1073/pnas.1111841109.

de Wijs, G., G. Kresse, L. Vočadlo, D. Dobson, D. Alfe, M. Gillan, and G. Price (1998). The viscosity of liquid iron at the physical conditions of the Earth's core. Nature 392(6678), 805-807. doi: 10.1038/33905.

de Wijs, G., G. Kresse, L. Vočadlo, D. Dobson, and Others (1998). The viscosity of liquid iron at the physical conditions of the Earth's core. Nature 392(6678), 805. doi: $10.1038 / 33905$.

der Hilst, R., M. De Hoop, P. Wang, S. Shim, P. Ma, and L. Tenorio (2007). Seismostratigraphy and thermal structure of Earth's core-mantle boundary region. Science 315(5820), 1813-1817. doi: 10.1126/science.1137867.

Deuss, A. (2014). Heterogeneity and anisotropy of Earth's inner core. Annual Review of Earth and Planetary Sciences 42, 103-126. doi: 10.1146/annurev-earth-060313-054658.

Dormy, E. (1997). Modélisation numérique de la dynamo terrestre.

Dormy, E., P. Cardin, and D. Jault (1998). MHD flow in a slightly differentially rotating spherical shell, with conducting inner core, in a dipolar magnetic field. Earth and Planetary Science Letters 160(1-2), 15-30. doi: 10.1016/S0012-821X(98)00078-8.

Dumberry, M. (2007). Geodynamic constraints on the steady and time-dependent inner core axial rotation. Geophysical Journal International 170(2), 886-895. doi: 10.1111/j.1365246X.2007.03484.x. 
Dziewonski, A. and D. Anderson (1981). Preliminary reference Earth model. Physics of the Earth and Planetary Interiors 25(4), 297-356. doi: 10.1016/0031-9201(81)90046-7.

Elsasser, W. (1946a). Induction effects in terrestrial magnetism part I. Theory. Physical Review 69(3-4), 106. doi: 10.1103/PhysRev.69.106.

Elsasser, W. (1946b). Induction effects in terrestrial magnetism part II. The secular variation. Physical Review 70(3-4), 202. doi: 10.1103/PhysRev.72.821.

Eymin, C. and G. Hulot (2005). On core surface flows inferred from satellite magnetic data. Physics of the Earth and Planetary Interiors 152(3), 200-220. doi: 10.1016/j.pepi.2005.06.009.

Ferraro, V. (1937). The non-uniform rotation of the sun and its magnetic field. Monthly Notices of the Royal Astronomical Society 97, 458-472. doi: 10.1093/mnras/97.6.458.

Finlay, C. and A. Jackson (2003). Equatorially dominated magnetic field change at the surface of Earth's core. Science 300, 2084-2086. doi: 10.1126/science.1083324.

Finlay, C., S. Maus, C. Beggan, T. Bondar, A. Chambodut, T. Chernova, A. Chulliat, V. Golovkov, B. Hamilton, M. Hamoudi, and Others (2010). International geomagnetic reference field: the eleventh generation. Geophysical Journal International 183(3), 1216-1230. doi: 10.1111/j.1365-246X.2010.04804.x.

Forte, A. and R. Peltier (1991). Viscous flow models of global geophysical observables: 1. Forward problems. Journal of Geophysical Research: Solid Earth 96(B12), 20131-20159. doi: 10.1029/91JB01709.

Friis-Christensen, E., H. Lühr, and G. Hulot (2006). Swarm: A constellation to study the Earth's magnetic field. Earth, Planets and Space 58(4), 351-358. doi: 10.1186/BF03351933.

Gillet, N., D. Jault, E. Canet, and A. Fournier (2010). Fast torsional waves and strong magnetic field within the Earth's core. Nature 465(7294), 74-77. doi: 10.1038/nature09010.

Gillet, N., M. Pais, and D. Jault (2009). Ensemble inversion of time-dependent core flow models. Geochemistry, Geophysics, Geosystems 10(6). doi: 10.1029/2008GC002290.

Glatzmaier, G. and P. Roberts (1995). A three-dimensional convective dynamo solution with rotating and finitely conducting inner core and mantle. Physics of the Earth and Planetary Interiors 91 (1-3), 63-75. doi: 10.1016/0031-9201(95)03049-3.

Glatzmaier, G. and P. Roberts (1996). Rotation and magnetism of earth's inner core. Science 274(5294), 1887-1891. doi: 10.1126/science.274.5294.1887.

Gleason, A. and W. Mao (2013). Strength of iron at core pressures and evidence for a weak Earth's inner core. Nature Geoscience 6(7), 571-574. doi: 10.1038/NGEO1808. 
Gomi, H., K. Ohta, K. Hirose, S. Labrosse, R. Caracas, M. Verstraete, and J. Hernlund (2013). The high conductivity of iron and thermal evolution of the Earth's core. Physics of the Earth and Planetary Interiors 224, 88-103. doi: 10.1016/j.pepi.2013.07.010.

Grayver, A., N. Schnepf, A. Kuvshinov, T. Sabaka, C. Manoj, and N. Olsen (2016). Satellite tidal magnetic signals constrain oceanic lithosphere-asthenosphere boundary. Science advances 2(9), e1600798. doi: 10.1126/sciadv.1600798.

Gross, R. (2001). A combined length-of-day series spanning 1832-1997: LUNAR97. Physics of the Earth and Planetary Interiors 123(1), 65-76. doi: 10.1016/S0031-9201(00)00217-X.

Gross, R. (2007). Earth rotation variations-long period. Treatise on Geophysics 3, 239-294. doi: 10.1016/B978-044452748-6/00057-2.

Gubbins, D. (1981). Rotation of the inner core. Journal of Geophysical Research 86(NB12), 1695-1699. doi: 10.1029/JB086iB12p11695.

Gubbins, D. (2001). The Rayleigh number for convection in the Earth's core. Physics of the Earth and Planetary Interiors 128(1), 3-12. doi: 10.1016/S0031-9201(01)00273-4.

Gutenberg, B. (1913). Uber die Konstitution des Erdinnern, erschlossen aus Erdbebenbeobachtungen. Phys. Z 14, 1217-1218.

Hale, G. (1908). On the probable existence of a magnetic field in sun-spots. The Astrophysical Journal 28, 315. doi: 10.1029/TE013i004p00159.

Halley, E. (1692). On the cause of the change in the variation of the magnetic needle, with a hypothesis of the structure of the internal parts of the Earth. Philosophical Transactions of the Royal Society of London 17.

Hide, R. (1969). Interaction between the Earth's liquid core and solid mantle. Nature 222(5198), 1055-1056. doi: 10.1038/2221055a0.

Holme, R. (1998). Electromagnetic core-mantle coupling - I. Explaining decadal changes in the length of day. Geophysical Journal International 132, 167-180. doi: 10.1046/j.1365246x.1998.00424.x.

Holme, R. and O. De Viron (2013). Characterization and implications of intradecadal variations in length of day. Nature 499(7457), 202. doi: 10.1038/nature12282.

Hori, K., C. Jones, and R. Teed (2015). Slow magnetic Rossby waves in the Earth's core. Geophysical Research Letters 42(16), 6622-6629. doi: 10.1002/2015GL064733.

Hulot, G., C. Eymin, B. Langlais, M. Mandea, and N. Olsen (2002). Small-scale structure of the geodynamo inferred from Oersted and Magsat satellite data. Nature 416(6881), 620-623. doi: 10.1038/416620a. 
Irving, J. and A. Deuss (2011). Hemispherical structure in inner core velocity anisotropy. Journal of Geophysical Research: Solid Earth 116(B4). doi: 10.1029/2010JB007942.

Jackson, A., A. Jonkers, M. Mandea, and A. Murray (2003). Earth's magnetic field in the early 19th century from French sources. Geochemistry, Geophysics, Geosystems 4(7). doi: 10.1029/2002GC000494.

Jackson, A., A. Jonkers, and M. Walker (2000). Four centuries of geomagnetic secular variation from historical records. Philosophical Transactions of the Royal Society of London A: Mathematical, Physical and Engineering Sciences 358(1768), 957-990. doi: 10.1098/rsta.2000.0569.

Jacobs, J. (1953). The Earth's inner core. Nature 172(4372), 297-298. doi: $10.1038 / 172297 \mathrm{a} 0$.

Jault, D., C. Gire, and J. Le Mouël (1988). Westward drift, core motions and exchanges of angular momentum between core and mantle. Nature 333(6171), 353-356. doi: $10.1038 / 333353 \mathrm{a} 0$.

Jault, D. and J. Le Mouël (1991). Exchange of angular momentum between the core and the mantle. Journal of Geomagnetism and Geoelectricity 43(2), 111-129. doi: 10.5636/jgg.43.111.

Jault, D. and J. Mouël (1989). The topographic torque associated with a tangentially geostrophic motion at the core surface and inferences on the flow inside the core. Geophysical \& Astrophysical Fluid Dynamics 48(4), 273-295. doi: 10.1080/03091928908218533.

Javoy, M., E. Kaminski, F. Guyot, D. Andrault, C. Sanloup, M. Moreira, S. Labrosse, A. Jambon, P. Agrinier, A. Davaille, and Others (2010). The chemical composition of the Earth: Enstatite chondrite models. Earth and Planetary Science Letters 293(3), 259-268. doi: 10.1016/j.epsl.2010.02.033.

Jeffreys, H. (1926). The Reflexion and Refraction of Elastic Waves. Geophysical Journal International 1(s7), 321-334. doi: 10.1111/j.1365-246X.1926.tb05380.x.

Jones, C. (2015). Thermal and Compositional Convection in the Outer Core. Treatise on Geophysics 8, 115-159. doi: 10.1016/B978-044452748-6/00130-9.

Kuang, W. and B. Chao (2001). Topographic Core-Mantle coupling in Geodynamo modeling. Geophysical Research Letters 28(9), 1871-1874. doi: 10.1029/2000GL012237.

Labrosse, S. (2015). Thermal evolution of the core with a high thermal conductivity. Physics of the Earth and Planetary Interiors 247, 36-55. doi: 10.1016/j.pepi.2015.02.002.

Labrosse, S., J. Poirier, and J. Le Mouël (2001). The age of the inner core. Earth and Planetary Science Letters 190(3), 111-123. doi: 10.1016/S0012-821X(01)00387-9. 
Langel, R. (1987). The main field. Journal of Geomagnetism and Geoelectricity 1, 249-512.

Langel, R., R. Estes, and G. Mead (1982). Some new methods in geomagnetic field modeling applied to the 1960-1980 epoch. Journal of Geomagnetism and Geoelectricity 34(6), 327-349. doi: 10.5636/jgg.34.327.

Larmor, J. (1919). How could a rotating body such as the Sun become a magnet. Report of the British Association for the Advancement of Science 159, 412.

Laske, G. and G. Masters (1999). Limits on differential rotation of the inner core from an analysis of the Earth's free oscillations. Nature 402(6757), 66-69. doi: 10.1038/47011.

Laske, G. and G. Masters (2003). The earth's free oscillations and the differential rotation of the inner core. Earth's Core: Dynamics, Structure, Rotation 31, 5-21. doi: 10.1029/GD031p0005.

Lehmann, I. (1936). Publications du Bureau Central Sismologique International. A14, $87-115$.

Lesur, V., I. Wardinski, M. Hamoudi, and M. Rother (2010). The second generation of the GFZ reference internal magnetic model: GRIMM-2. Earth, Planets and Space 62(10), 6. doi: $10.5047 /$ eps.2010.07.007.

Lesur, V., I. Wardinski, M. Rother, and M. Mandea (2008). GRIMM: the GFZ Reference Internal Magnetic Model based on vector satellite and observatory data. Geophysical Journal International 173(2), 382-394. doi: 10.1111/j.1365-246X.2008.03724.x.

Lhuillier, F., J. Aubert, and G. Hulot (2011). Earth's dynamo limit of predictability controlled by magnetic dissipation. Geophysical Journal International 186(2), 492-508. doi: $10.1139 / \mathrm{p} 01-116$.

Livermore, P., R. Hollerbach, and A. Jackson (2013). Electromagnetically driven westward drift and inner-core superrotation in Earth's core. Proceedings of the National Academy of Sciences 110(40), 15914-15918. doi: 10.1073/pnas.1307825110.

Lowes, F. (1974). Spatial power spectrum of the main geomagnetic field, and extrapolation to the core. Geophysical Journal International 36(3), 717-730. doi: 10.1111/j.1365246X.1974.tb00622.x.

Mäkinen, A. and A. Deuss (2011). Global seismic body-wave observations of temporal variations in the Earth's inner core, and implications for its differential rotation. Geophysical Journal International 187(1), 355-370. doi: 10.1111/j.1365-246X.2011.05146.x.

McNamara, A. and S. Zhong (2005). Thermochemical structures beneath Africa and the Pacific Ocean. Nature 437(7062), 1136. doi: 10.1038/nature04066. 
Monnereau, M., M. Calvet, L. Margerin, and A. Souriau (2010). Lopsided growth of Earth's inner core. Science 328(5981), 1014-1017. doi: 10.1126/science.1186212.

Morelli, A. and A. Dziewonski (1987). Topography of the core-mantle boundary and lateral homogeneity of the liquid core. Nature 325 (6106), 678-683. doi: 10.1038/325678a0.

Morelli, A., A. Dziewonski, and J. Woodhouse (1986). Anisotropy of the inner core inferred from PKIKP travel times. Geophysical Research Letters 13(13), 1545-1548. doi: 10.1029/GL013i013p01545.

Mound, J. and B. Buffett (2003). Interannual oscillations in length of day: implications for the structure of the mantle and core. Journal of Geophysical Research: Solid Earth 108(B7). doi: 10.1029/2002JB002054.

Mound, J. and B. Buffett (2006). Detection of a gravitational oscillation in length-of-day. Earth and Planetary Science Letters 243(3-4), 383-389. doi: 10.1016/j.epsl.2006.01.043.

Murakami, M., K. Hirose, K. Kawamura, N. Sata, and Y. Ohishi (2004). Post-perovskite phase transition in MgSiO3. Science 304(5672), 855-858. doi: 10.1126/science.1095932.

Nagata, T. (1962). Two main aspects of geomagnetic secular variation-westward drift and non-drifting components. In Proc. Benedum Earth Magnetism Symp, Volume 39, pp. 55.

Niu, F. and L. Wen (2001). Hemispherical variations in seismic velocity at the top of the Earth's inner core. Nature 410(6832), 1081. doi: 10.1038/35074073.

Ohta, K., R. Cohen, K. Hirose, K. Haule, K. Shimizu, and Y. Ohishi (2012). Experimental and Theoretical Evidence for Pressure-Induced Metallization in FeO with Rocksalt-Type Structure. Physical Review Letters 108(2). doi: 10.1103/PhysRevLett.108.026403.

Ohta, K., K. Fujino, Y. Kuwayama, T. Kondo, K. Shimizu, and Y. Ohishi (2014). Highly conductive iron-rich $(\mathrm{Mg}, \mathrm{Fe}) \mathrm{O}$ magnesiowüstite and its stability in the Earth's lower mantle. Journal of Geophysical Research: Solid Earth 119(6), 4656-4665. doi: 10.1002/2014JB010972.

Ohta, K., S. Onoda, K. Hirose, R. Sinmyo, K. Shimizu, N. Sata, Y. Ohishi, and A. Yasuhara (2008). The electrical conductivity of post-perovskite in Earth's D" layer. Science 320 (5872), 89-91. doi: 10.1126/science.1155148.

Oldham, R. (1906). The constitution of the interior of the Earth, as revealed by earthquakes. Quarterly Journal of the Geological Society 62(1-4), 456-475. doi: 10.1144/GSL.JGS.1906.062.01-04.21.

Olsen, N., H. Lühr, T. Sabaka, M. Mandea, M. Rother, L. Tøffner-Clausen, and S. Choi (2006). CHAOS-a model of the Earth's magnetic field derived from CHAMP, Ørsted, and 
SAC-C magnetic satellite data. Geophysical Journal International 166(1), 67-75. doi: 10.1111/j.1365-246X.2006.02959.x.

Olsen, N. and M. Mandea (2008). Rapidly changing flows in the Earth's core. Nature Geoscience 1(6), 390. doi: 10.1038/ngeo203.

Olsen, N., M. Mandea, T. Sabaka, and L. Tøffner-Clausen (2009). CHAOS-2-a geomagnetic field model derived from one decade of continuous satellite data. Geophysical Journal International 179(3), 1477-1487. doi: 10.1111/j.1365-246X.2009.04386.x.

Olsen, N., M. Mandea, T. Sabaka, and L. Tøffner-Clausen (2010). The CHAOS-3 geomagnetic field model and candidates for the 11th generation IGRF. Earth, Planets and Space 62(10), 1. doi: 10.5047/eps.2010.07.003.

Olson, P. and J. Aurnou (1999). A polar vortex in the Earth's core. Nature 402(6758), 170-173. doi: 10.1038/46017.

Pais, M. and D. Jault (2008). Quasi-geostrophic flows responsible for the secular variation of the Earth's magnetic field. Geophysical Journal International 173(2), 421-443. doi: 10.1111/j.1365-246X.2008.03741.x.

Parker, E. (1955). Hydromagnetic Dynamo Models. The Astrophysical Journal 122, 293. doi: $10.1086 / 146087$.

Parker, R. (1994). Geophysical inverse theory. Princeton university press.

Pichon, G., J. Aubert, and A. Fournier (2016). Coupled dynamics of Earth's geomagnetic westward drift and inner core super-rotation. Earth and Planetary Science Letters 437, 114-126. doi: 10.1016/j.epsl.2016.01.004.

Poirier, J. (1988). Transport properties of liquid metals and viscosity of the Earth's core. Geophysical Journal International 92(1), 99-105. doi: 10.1111/j.1365-246X.1988.tb01124.x.

Poupinet, G., R. Pillet, and A. Souriau (1983). Possible heterogeneity of the Earth's core deduced from PKIKP travel times. Nature 305(5931), 204-206. doi: 10.1038/305204a0.

Poupinet, G., A. Souriau, and O. Coutant (2000). The existence of an inner core superrotation questioned by teleseismic doublets. Physics of the Earth and Planetary Interiors 118(1), 77-88. doi: 10.1016/S0031-9201(99)00129-6.

Pozzo, M., C. Davies, D. Gubbins, and D. Alfe (2012). Thermal and electrical conductivity of iron at Earth's core conditions. Nature 485(7398), 355-U99. doi: 10.1038/nature11031.

Roberts, P. (1968). On the thermal instability of a rotating-fluid sphere containing heat sources. Philosophical Transactions of the Royal Society of London A: Mathematical, Physical and Engineering Sciences 263(1136), 93-117. doi: 10.1098/rsta.1968.0007. 
Roberts, P. (1988). On topographic core-mantle coupling. Geophysical \& Astrophysical Fluid Dynamics 44(1-4), 181-187. doi: 10.1080/03091928808208884.

Roberts, P. and J. Aurnou (2012). On the theory of core-mantle coupling. Geophysical \& Astrophysical Fluid Dynamics 106(2), 157-230. doi: 10.1080/03091929.2011.589028.

Roberts, P. and E. King (2013). On the genesis of the Earth's magnetism. Reports on Progress in Physics 76(9). doi: 10.1088/0034-4885/76/9/096801.

Roberts P and S. Scott (1965). On analysis of secular variation .1. A Hydromagnetic constraint- Theory. Journal of Geomagnetism and Geoelectricity 17(2), 137-\&. doi: 10.5636/jgg.17.137.

Rochester, M. (1960). Geomagnetic westward drift and irregularities in the earth's rotation. Philosophical Transactions of the Royal Society of London A: Mathematical, Physical and Engineering Sciences 252(1018), 531-555. doi: 10.1098/rsta.1960.0014.

Rochester, M. (1962). Geomagnetic core-mantle coupling. Journal of Geophysical Research 67(12), 4833-\&. doi: 10.1029/JZ067i012p04833.

Sakai, T., E. Ohtani, N. Hirao, and Y. Ohishi (2011). Stability field of the hcp-structure for $\mathrm{Fe}, \mathrm{Fe}-\mathrm{Ni}$, and Fe-Ni-Si alloys up to 3 Mbar. Geophysical research letters 38(9). doi: 10.1029/2011GL047178.

Schaeffer, N. (2013). Efficient spherical harmonic transforms aimed at pseudospectral numerical simulations. Geochemistry, Geophysics, Geosystems 14(3), 751-758. doi: 10.1002 /ggge.20071.

Schaeffer, N., D. Jault, H. Nataf, and A. Fournier (2017). Turbulent geodynamo simulations: a leap towards Earth's core . Geophysical Journal International (1), 1-29. doi: $10.1093 /$ gji/ggx265.

Schnepf, N., A. Kuvshinov, and T. Sabaka (2015). Can we probe the conductivity of the lithosphere and upper mantle using satellite tidal magnetic signals? Geophysical Research Letters 42(9), 3233-3239. doi: 10.1002/2015GL063540.

Sharrock, D. and J. Woodhouse (1998). Investigation of time dependent inner core structure by the analysis of free oscillation spectra. Earth, Planets and Space 50(11-12), 1013-1018. doi: 10.1186/BF03352195.

Shearer, P. and K. Toy (1991). PKP (BC) versus PKP (DF) differential travel times and aspherical structure in the geophysi inner core. Journal of Geophysical Research: Solid Earth 96(B2), 2233-2247. doi: 10.1029/90JB02370. 
Song, X. (2000). Joint inversion for inner core rotation, inner core anisotropy, and mantle heterogeneity. Journal of Geophysical Research 105(B4), 7931-7943. doi: 10.1029/1999JB900436.

Song, X. and G. Poupinet (2007). Inner core rotation from event-pair analysis. Earth and Planetary Science Letters 261(1), 259-266. doi: 10.1016/j.epsl.2007.06.034.

Song, X. and P. Richards (1996). Seismological evidence for differential rotation of the Earth's inner core. Nature 382(6588), 221-224. doi: 10.1038/382221a0.

Souriau, A. and M. Calvet (2015). The Earth's Cores. In A. Dziewonski and B. Romanowicz (Eds.), Treatise on Geophysics, Second Edition. 1.23 -Deep Earth Seismology, Volume 1. Elsevier.

Souriau, A. and G. Poupinet (2000). Inner core rotation: a test at the worldwide scale. Physics of the Earth and Planetary Interiors 118(1), 13-27. doi: 10.1016/S00319201(99)00131-4.

Sreenivasan, B. and C. Jones (2006). Azimuthal winds, convection and dynamo action in the polar regions of planetary cores. Geophysical \& Astrophysical Fluid Dynamics 100(4-5), 319-339. doi: 10.1080/03091920600807864.

Stewart, D., F. Busse, K. Whaler, and D. Gubbins (1995). Geomagnetism, Earth rotation and the electrical conductivity of the lower mantle. Physics of the Earth and Planetary Interiors 92(3), 199-214. doi: 10.1016/0031-9201(95)03035-4.

Stix, M. and P. Roberts (1984). Time-dependent electromagnetic core-mantle coupling. Physics of the Earth and Planetary Interiors 36(1), 49-60. doi: 10.1016/00319201(84)90098-0.

Stixrude, L. and R. Cohen (1995). High-pressure elasticity of iron and anisotropy of Earth's inner core. Science 267(5206), 1972. doi: 10.1126/science.267.5206.1972.

$\mathrm{Su}, \mathrm{W}$. and A. Dziewonski (1995). Inner core anisotropy in three dimensions. Journal of Geophysical Research: Solid Earth 100(B6), 9831-9852. doi: 10.1029/95JB00746.

Su, W., A. Dziewonski, and R. Jeanloz (1996). Planet within a planet: rotation of the inner core of Earth. Science 274(5294), 1883. doi: 10.1126/science.274.5294.1883.

Tanaka, S. (2010). Constraints on the core-mantle boundary topography from P4KP-PcP differential travel times. Journal of Geophysical Research 115. doi: 10.1029/2009JB006563.

Tanaka, S. and H. Hamaguchi (1997). Degree one heterogeneity and hemispherical variation of anisotropy in the inner core from PKP (BC)-PKP (DF) times. Journal of Geophysical Research: Solid Earth 102(B2), 2925-2938. doi: 10.1029/96JB03187. 
Tarduno, J., R. Cottrell, W. Davis, F. Nimmo, and R. Bono (2015). A Hadean to Paleoarchean geodynamo recorded by single zircon crystals. Science 349(6247), 521-524. doi: 10.1126/science.aaa9114.

Tateno, S., K. Hirose, T. Komabayashi, H. Ozawa, and Y. Ohishi (2012). The structure of Fe-Ni alloy in Earth's inner core. Geophysical Research Letters 39(12). doi: 10.1029/2012GL052103.

Tateno, S., K. Hirose, Y. Ohishi, and Y. Tatsumi (2010). The structure of iron in Earth's inner core. Science 330(6002), 359-361. doi: 10.1126/science.1194662.

Terasaki, H., A. Suzuki, E. Ohtani, K. Nishida, T. Sakamaki, and K. Funakoshi (2006). Effect of pressure on the viscosity of Fe-S and Fe-C liquids up to $16 \mathrm{GPa}$. Geophysical Research Letters 33(22). doi: 10.1029/2006GL027147.

Thébault, E., C. Finlay, P. Alken, C. Beggan, E. Canet, A. Chulliat, B. Langlais, V. Lesur, F. Lowes, C. Manoj, and Others (2015). Evaluation of candidate geomagnetic field models for IGRF-12. Earth, Planets and Space 67(1), 112. doi: 10.1186/s40623-015-0273-4.

Thébault, E., C. Finlay, C. Beggan, P. Alken, J. Aubert, O. Barrois, F. Bertrand, T. Bondar, A. Boness, L. Brocco, and Others (2015). International geomagnetic reference field: the 12th generation. Earth, Planets and Space 67(1), 79. doi: 10.1186/s40623-015-0228-9.

Tkalčić, H., Y. Mallory, T. Bodin, S. Ngo, and M. Sambridge (2013). The shuffling rotation of the Earth's inner core revealed by earthquake doublets. Nature Geoscience 6(6), 497-502. doi: 10.1038/NGEO1813.

Tromp, J. (1993). Support for anisotropy of the Earth's inner core from free oscillations. Nature 366 (6456), 678-681. doi: 10.1038/366678a0.

Tyler, R., S. Maus, and H. Lühr (2003). Satellite observations of magnetic fields due to ocean tidal flow. Science 299(5604), 239-241. doi: 10.1126/science.1078074.

Vestine, E. and A. Kahle (1968). The westward drift and geomagnetic secular change. Geophysical Journal International 15(1-2), 29-37. doi: 10.1111/j.1365-246X.1968.tb05743.x.

Vočadlo, L., D. Alfè, M. Gillan, I. Wood, and Others (2003). Possible thermal and chemical stabilization of body-centred-cubic iron in the Earth's core. Nature 424(6948), 536. doi: 10.1038/nature01829.

Vočadlo, L., J. Brodholt, D. Alfè, M. Gillan, and G. Price (2000). Ab initio free energy calculations on the polymorphs of iron at core conditions. Physics of the Earth and Planetary Interiors 117(1), 123-137. doi: 10.1016/S0031-9201(99)00092-8. 
Woodhouse, J., D. Giardini, and X. Li (1986). Evidence for inner core anisotropy from free oscillations. Geophysical Research Letters 13(13), 1549-1552. doi: 10.1029/GL013i013p01549.

Yukutake, T. (1962). The westward drift of the magnetic field of the Earth.

Zatman, S. (2003). Decadal oscillations of the Earth's core, angular momentum exchange, and inner core rotation. Earth's Core: Dynamics, Structure, Rotation, 233-240. doi: 10.1029/GD031p0233.

Zhang, J., X. Song, Y. Li, P. Richards, X. Sun, and F. Waldhauser (2005). Inner core differential motion confirmed by earthquake waveform doublets. Science 309(5739), 1357-1360. doi: 10.1126/science.1113193. 


\title{
Dynamique rotationnelle couplée de la dérive géomagnétique vers l'ouest et de la super-rotation de la graine terrestre Guillaume Pichon
}

\begin{abstract}
This PhD work focuses on the rotational dynamics of the coupled inner core - outer core - mantle system. The conservation of the angular momentum our coupled Earth model indeed involves two direct electromagnetic torques at the fluid core boundaries and a remote gravitational torque between the inner core and the mantle. The rotational dynamics is described by four typical shears and studied in convective numerical simulations of the geodynamo which are able to reproduce the main characteristics of the geomagnetic field and its secular variation. The latter is mainly embodied by the westward drift of magnetic flux patches at the CMB, concentrated on the equator of the Atlantic hemisphere, and is well documented for the last four centuries. We provide constrains on the inner core differential rotation by expressing its link to the geomagnetic westward drift. This is performed through the formulation and the validation of dynamical electromagnetic torque models.In the long-term state, the global shear in the fluid outer core is distributed between the westward drift and the differential rotation of the inner core, in proportions controlled by the state of couplings. As a present day estimate of this shear is close to the observed westward drift, we conclude there is no differential rotation of the inner core on time-average. In the time-dependent state, we observed that the strength of gravitational coupling is the dominant parameter. This places limit on the decadal fluctuations of the inner core differential rotation, which should not exceed a few hundredths of degree per year.
\end{abstract}

Keywords : geodynamo, geomagnetism, wesward drift, inner core, super-rotation, couplings, numerical simulations.

\section{Résumé}

Ce travail de thèse se concentre sur la dynamique rotationnelle du système couplé graine, noyau externe et manteau. Notre modèle inclut en effet deux couples électromagnétiques directs aux limites du noyau fluide et un couple gravitationnel à distance entre le noyau interne et le manteau. La dynamique rotationnelle est décrite par quatre cisaillements typiques et étudiés dans des simulations numériques de la géodynamo reproduisant les principales caractéristiques du champ magnétique terrestre et de sa variation séculaire. Celle-ci est principalement représentée par la dérive géomagnétique vers l'ouest de taches de flux magnétique à la CMB, concentrée à l'équateur de l'hémisphère Atlantique, et bien documentée pour les quatre derniers siècles. Nous fournissons des contraintes sur la rotation différentielle du noyau interne en exprimant son lien avec la dérive géomagnétique vers l'ouest. Ceci est réalisé par la formulation et la validation de modèles dynamiques de couples électromagnétiques. Au long terme, le cisaillement global dans le noyau fluide est réparti entre la dérive vers l'ouest et la rotation différentielle de la graine, dans des proportions contrôlées par l'état des couplages. Puisqu'une estimation actuelle de ce cisaillement est proche de la vitesse de la dérive géomagnétique vers l'ouest, nous concluons que la rotation différentielle moyenne de la graine est proche de zéro. En ce qui concerne ses fluctuations, l'intensité du couplage gravitationnel est le paramètre dominant. Cette observation place alors une limite sur les fluctuations décennales de la rotation différentielle de la graine, qui ne devraient pas excéder quelques centièmes de degré par an.

Mots clés : geodynamo, geomagnétisme, dérive vers l'ouest, noyau interne, superrotation, magnétohydrodynamique, couplage, simulations numériques. 